\title{
Algebraic higher symmetry and categorical symmetry: A holographic and entanglement view of symmetry
}

\author{
Liang Kong, ${ }^{1,2}$ Tian Lan $\odot,{ }^{3}$ Xiao-Gang Wen $\odot,{ }^{4}$ Zhi-Hao Zhang $\odot,{ }^{5,1}$ and Hao Zheng ${ }^{1,2,6}$ \\ ${ }^{1}$ Shenzhen Institute for Quantum Science and Engineering, Southern University of Science and Technology, \\ Shenzhen 518055, People's Republic of China \\ ${ }^{2}$ Guangdong Provincial Key Laboratory of Quantum Science and Engineering, Southern University of Science and Technology, \\ Shenzhen 518055, People's Republic of China \\ ${ }^{3}$ Institute for Quantum Computing, University of Waterloo, Waterloo, Ontario N2L 3G1, Canada \\ ${ }^{4}$ Department of Physics, Massachusetts Institute of Technology, Cambridge, Massachusetts 02139, USA \\ ${ }^{5}$ School of Mathematical Sciences, University of Science and Technology of China, Hefei 230026, People's Republic of China \\ ${ }^{6}$ Department of Mathematics, Peking University, Beijing 100871, People's Republic of China
}

(Received 30 June 2020; revised 16 September 2020; accepted 18 September 2020; published 15 October 2020)

\begin{abstract}
A global symmetry ( 0 -symmetry) in an $n$-dimensional space acts on the whole space. A higher symmetry acts on closed submanifolds (i.e., loops and membranes, etc.), and those transformations form a higher group. In this paper, we introduce the notion of algebraic higher symmetry, which generalizes higher symmetry and is beyond higher group. We show that an algebraic higher symmetry in a bosonic system in $n$-dimensional space is characterized and classified by a local fusion n-category. We find that, when restricted to its symmetric subHilbert space, an algebraic higher symmetry can be fully characterized by a noninvertible gravitational anomaly (i.e., a topological order in one higher dimension). Thus, we also refer to a noninvertible gravitational anomaly as categorical symmetry to stress its connection to symmetry. This provides a holographic and entanglement view of symmetries. For a system with a categorical symmetry, its gapped state must spontaneously break part (not all) of the symmetry, and the state with the full symmetry must be gapless. Using such a holographic point of view, we obtain (1) the gauging of the algebraic higher symmetry; (2) the classification of anomalies for an algebraic higher symmetry; (3) the equivalence between classes of systems, with different (potentially anomalous) algebraic higher symmetries or different sets of low-energy excitations, as long as they have the same categorical symmetry; and (4) the classification of gapped liquid phases for bosonic and/or fermionic systems with a categorical symmetry, as gapped boundaries of a topological order in one higher dimension (that corresponds to the categorical symmetry). This classification includes symmetry protected trivial (SPT) orders and symmetry enriched topological (SET) orders with an algebraic higher symmetry.
\end{abstract}

DOI: 10.1103/PhysRevResearch.2.043086

\section{INTRODUCTION}

The notion of a symmetry plays a very important role in physics. A quantum system living on $n$-dimensional space ${ }^{1}$ $M^{n}$ is defined by a vector space $\mathcal{V}$ formed by wave functions on $M^{n}$ and a Hamiltonian $H$. A symmetry in such a system is a set of linear constraints on the allowed Hamiltonians. Since the Hamiltonian is always a sum of local operators $H=\sum_{x} O_{x}$, we can also more precisely describe a symmetry as a set of linear constraints on the allowed local operators.

\footnotetext{
${ }^{1}$ Here, a $n$-dimensional space $M^{n}$ actually means a triangulation of $n$-dimensional manifold. So, $M^{n}$ should be viewed as a $n$-dimensional simplicial complex. In this paper, we mainly consider discrete lattice systems.

Published by the American Physical Society under the terms of the Creative Commons Attribution 4.0 International license. Further distribution of this work must maintain attribution to the author(s) and the published article's title, journal citation, and DOI.
}

Those allowed local operators are called symmetric local operators and they form an algebra of symmetric local operators. A symmetric Hamiltonian is a sum of symmetric local operators. The algebra of symmetric local operators contains all the information about the symmetry and represents a very general way to describe the symmetry. In this paper, we will use this point of view to show that a symmetry in $n$-dimensional space is described as a local fusion n-category.

By a "symmetry," we usually mean a global symmetry, where we have a set of unitary operators $W_{\alpha}$, labeled by $\alpha$, acting on the whole space $M^{n}$ (i.e., a symmetry transformation), which gives rise to the following linear constraint on the local operators $W_{\alpha} O_{x}=O_{x} W_{\alpha}$. If one digs deeper, however, one finds that there are in fact several different kinds of global symmetries. In quantum field theories, we have anomalyfree global symmetries (gaugeable global symmetries) and anomalous global symmetries (non-gaugeable global symmetries or 't Hooft anomalies [1]). In lattice systems, we have on-site symmetries [where the symmetry transformation has a composition in terms of operators $W_{\alpha}(\boldsymbol{x})$ that acts only on 
lattice site labeled by $\left.\boldsymbol{x}: W_{\alpha}=\otimes_{x} W_{\alpha}(\boldsymbol{x})\right]$ and non-on-site symmetries [2,3].

These different kinds of global symmetries are closely related. Consider a low-energy effective field theory of a lattice model. The on-site symmetries in the lattice model becomes the anomaly-free global symmetries in the effective field theory, since the lattice on-site symmetry is always gaugeable. The non-on-site symmetries in the lattice model become the anomalous global symmetries in the effective field theory [3]. For the symmetries related to spacial transformation, such as the lattice translation symmetry and point group symmetry, sometimes they become anomalous symmetry in the effective field theory, and sometimes they are anomaly-free. In this paper, we consider only internal symmetries instead of symmetries related to spatial transformations.

There are also gauge symmetries in field theories and lattice theories, but they are not symmetries in quantum systems and should not be called symmetry at all.

Recently, in Ref. [4], the notion of a global symmetry was generalized to a $k$-form symmetry, which acts on all closed subspaces of codimension $k$ and becomes the identity operator if the closed subspaces are contractible. It was stressed that many results and intuitions for global symmetries (the 0 -form symmetries) can be extended to higher form symmetries.

In fact, closely related higher symmetries have been studied earlier (but under various different names, such as logical operator, gauge-like symmetry, etc.), where exactly solvable lattice Hamiltonians commuting with all closed string and/or membrane operators were constructed to realize topological orders [5-11]. We call a lattice symmetry generated by a $k$-codimensional operator as a $k$-symmetry, where the codimension in this paper is defined with respect to the space dimension. Similar to a $k$-form symmetry, a $k$-symmetry acts on closed subspaces of codimension $k$, but it does not become the identity operator when the closed subspaces are contractible. A higher symmetry is a symmetry in a lattice model. A higher symmetry reduces to a higher form symmetry in the ground-state subspaces (i.e., in low-energy effective topological quantum field theory). Our local fusion higher category description of symmetry includes those higher symmetries.

The emergence of higher symmetries was also studied before (again under different names, such as string-operators satisfying zero law) [12], where it was found that, unlike usual global symmetry (i.e., 0 -symmetry), the emergent higher symmetries cannot be destroyed by any local perturbations. Such a topological robustness was used to show that the emergent gapless $U(1)$ gauge bosons are robust against any local perturbations - a topological version of Goldstone theorem [12]. See Refs. [13-18] for some recent discussions of lattice higher symmetries, their emergence, anomalies, and a classification of associated higher symmetry protected phases on lattice.

In this work, we study another kind of symmetries that is beyond higher groups. We refer to these symmetries as algebraic higher symmetries and refer to higher groups as group-like higher symmetries. Algebraic higher symmetries include group-like higher symmetries as special cases.

Group-like higher symmetries and algebraic higher symmetries can both be generated by $p$-dimensional operators
$W_{\alpha}\left(S^{p}\right)$ labeled by an index $\alpha$ and $S^{p}$ (a $p$-dimensional closed submanifold), and $W_{\alpha}\left(S^{p}\right)$ only acts on the degrees of freedom near $S^{p}$. For a group-like higher symmetry, the $k$-dimensional operators satisfy a group-like algebra

$$
W_{\alpha}\left(S^{p}\right) W_{\beta}\left(S^{p}\right)=W_{\gamma}\left(S^{p}\right),
$$

while for an algebraic higher symmetry, they may satisfy a more general multiplication algebra [19],

$$
W_{\alpha}\left(S^{p}\right) W_{\beta}\left(S^{p}\right)=\sum_{\gamma} N_{\alpha \beta}^{\gamma} W_{\gamma}\left(S^{p}\right) .
$$

In this case, the symmetry generator $W_{\alpha}\left(S^{p}\right)$ may be neither invertible nor unitary. Such kind of algebraic symmetries was studied in $1+1 \mathrm{D}$ conformal field theory via noninvertible defect lines (where invertible defect lines are known to connect to symmetry) [20-23]. We believe that local fusion higher categories classify the anomaly-free algebraic higher symmetries, while anomalous algebraic higher symmetries are described by generic higher categories.

In Sec. IV, we discuss an example, a lattice model described by a Hamiltonian $H$, where the above algebraic higher symmetry does show up, i.e.,

$$
W_{\alpha}\left(S^{p}\right) H=H W_{\alpha}\left(S^{p}\right) .
$$

Then, in Secs. VI and V, we discuss unbroken anomaly-free algebraic higher symmetry from a point of view of trivial symmetric product state and local fusion higher category. In Sec. VIE, we show that an algebraic higher symmetry can be fully described by a noninvertible gravitational anomaly [24] (which is the same as a topological order in one higher dimension [3,25-27]), and this is a very useful way to view symmetry. To stress its relation to symmetry, we also refer to noninvertible gravitational anomaly (i.e., topological order in one higher dimension) as categorical symmetry [19]. In Sec. VII B, we obtain a classification of gapped liquid phases for systems with a categorical symmetry. It includes the classification of symmetry protected trivial (SPT) phases and that of symmetry enriched topological (SET) phases for algebraic higher symmetry. In Sec. VIII, we describe the emergence of categorical symmetries from topological orders, when the excitations have a large separation of energy.

The main point of this paper is about anomaly-free algebraic higher symmetries that are generally described and classified by local fusion higher categories. We also study topological orders with algebraic higher symmetries. Our approach is based on fusion higher category description of topological orders [25-31], which will be reviewed, clarified, and expanded in Sec. III. A brief summary of higher category description of topological orders can also be found in the first few subsections of Sec. II. This section tries to summarize the results of this paper for physics readers.

We have a more mathematical version of this paper published as Ref. [31]. The present paper contains more physical results and has more physical discussions.

We remark that the precise definitions of fusion higher categories and local fusion higher categories are difficult due 
to the lack of the universally accepted and well-developed model for weak $n$-categories. In this paper, we try to give a physical definition via the notion of topological orders. Many related concepts for topological order in arbitrary dimensions and for higher categories are discussed this way in Sec. III.

We like to point out that the physical definition of topological orders given in Ref. [32] is based on microscopic lattice models. In fact, many physical concepts are defined via microscopic lattice models, and we refer to those kinds of definition as microscopic definitions. There are also many physical concepts which are defined via macroscopic measurements, such as superfluidity defined via vanishing viscosity and quantization of vorticity. We refer to those kinds of definitions as macroscopic definitions. In this respect, the definitions in mathematics are macroscopic definitions, so mathematical definitions are closer to physical experiments. Some notions in symmetry and topological orders are defined microscopically, such as topological excitations [33-35], long-range entanglement [32], the characterization of algebraic higher symmetry by (2), etc. Some other notions are defined microscopically, such as topological degeneracy and the associated modular transformations [36-38], etc. A lot of the effort of this work is to convert microscopic definitions to macroscopic definitions, when possible. We will use ${ }^{\text {ph }}$ to indicate the microscopic definitions. Most results of this paper are presented via propositions. Those results are physical results based various physical arguments and beliefs.

Throughout this work, we use $n$ d to denote the spacial dimension and $(n+1) \mathrm{D}$ to denote the spacetime dimension, and the following convention of notations:

(1) $n \mathrm{D}$ topological orders: $\mathrm{A}^{n}, \mathrm{~B}^{n}, \mathrm{C}^{n}$ (mathsf font);

(2) fusion $n$-categories: $\mathcal{A}^{n}, \mathcal{B}^{n}, \mathcal{C}^{n}$ (mathcal font);

(3) braided fusion $n$-categories: $\mathcal{A}^{n}, \mathcal{B}^{n}, \mathrm{C}^{n}$ (euscript font).

Throughout this paper, superscripts always mean the spacetime dimension, or level of higher category. Also in this paper, we only consider finite algebraic higher symmetry. We mostly consider bosonic systems, except in Secs. VII C 3, VII C 4, and VII C 6. When we say, for example, SPT orders, we mean SPT orders in a bosonic system. In Secs. VIIC 3, VIIC 4, and VIIC 6, our results apply to both bosonic and fermionic systems, and even anyonic systems, via a more general notion of algebraic higher symmetry.

\section{SUMMARY OF MAIN RESULTS}

Since the main text of this paper is quite mathematical, in this section, we summarize the main results in less rigorous physical terms and introduce concepts and notations along the way.

\section{A. Category of topological orders}

First, let us introduce some concepts and notations about topological order. Let $\mathfrak{M}^{n+1}$, called the moduli space, be the space of Hamiltonians that support a gapped liquid ground state $[39,40]$. An element in $\pi_{0}\left(\mathfrak{M}^{n+1}\right)$ is a gapped liquid phase, i.e., a topological order, which is denoted by $\mathrm{M}^{n+1}$. So, a topological order is a gapped liquid phase $[39,40]$ (see Definition 8, which is a microscopic definition).
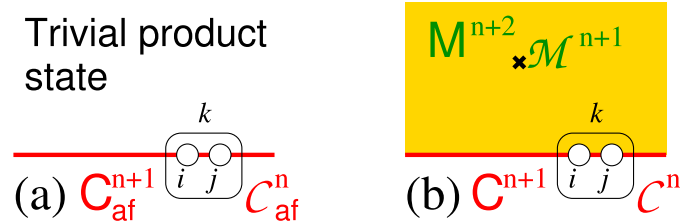

FIG. 1. (a) An anomaly-free topological order $\mathrm{C}_{\mathrm{af}}^{n+1} \in \mathrm{TO}_{\mathrm{af}}^{n+1}$ in $(n+1)$-dimensional spacetime can be realized on lattice in the same dimension, which can also be viewed as a boundary of a trivial product state in one higher dimension. The excitations in $\mathrm{C}_{\mathrm{af}}^{n+1}$ are described by fusion $n$-category $\mathcal{C}_{\text {af }}^{n}$. (b) An anomalous topological order $\mathrm{C}^{n+1} \in \mathrm{TO}^{n+1}$ in $(n+1)$-dimensional spacetime can be realized as a boundary of an anomaly-free topological order $\mathrm{M}^{n+2}$ in one higher dimension. The excitations in $\mathrm{C}^{n+1}$ are described by fusion $n$-category $\mathcal{C}^{n}$. The excitations in $\mathrm{M}^{n+2}$ are described by fusion $(n+1)$-category $\mathcal{M}^{n+1}$.

A topological order in $n$-dimensional space is roughly described macroscopically by the following data [25,26]:

(1) the codimension-1, codimension-2, etc., excitations above the gapped liquid state (see Fig. 1);

(2) the domain walls between two high dimensional excitations;

(3) the domain walls connecting to other topological orders (see Fig. 2);

(4) the monoid formed by the stacking topological orders.

Roughly, the data in the first two items describes a fusion $n$-category $\mathcal{M}^{n}$, which is a partial description of topological order $\mathrm{M}^{n+1}$. If we add the data in the third and fourth items to fusion $n$-category $\mathcal{M}^{n}$, we get a full description of the topological order $\mathrm{M}^{n+1}$ (i.e., a full description of gapped liquid phase).

To incorporate all the above data in one framework, we can put all those topological orders in $(n+1)$-dimensional spacetime together to form a category $\mathrm{TO}^{n+1}$ of $(n+1) \mathrm{D}$ topological orders [26] (see Sec. III F). It consists of a collection of topological orders (called the objects or 0-morphisms of the category), and 1-codimensional gapped domain walls between two (not necessarily different) topological orders (called 1-morphisms of the category), and 2-codimensional domain walls between 1-codimensional domain walls (called 2-morphisms), so on and so forth. The top morphisms are $(n+1)$-morphisms, which are local operators (satisfying certain symmetry constraints) acting on a spacetime point $(\boldsymbol{x}, t)$.
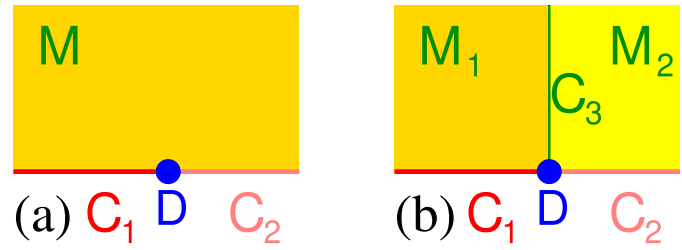

FIG. 2. (a) An anomaly-free domain wall $D$ between two (potentially anomalous) topological orders $\mathrm{C}_{1}$ and $\mathrm{C}_{2}$. (b) An anomalous domain wall $D$ between two (potentially anomalous) topological orders $\mathrm{C}_{1}$ and $\mathrm{C}_{2}$. $\mathrm{D}$ is a boundary of domain wall $\mathrm{C}_{3}$ which is a (potentially anomalous) topological order. 
The top morphisms can also be viewed as instantons in spacetime. The objects form a monoid under the stacking operation.

To be more precise, we distinguish two different categories: $\mathrm{TO}^{n+1}$ and $\mathrm{TO}_{\mathrm{af}}^{n+1}$. A topological order is called anomaly-free (i.e., in $\mathrm{TO}_{\mathrm{af}}^{n+1}$ ) if it can be realized by lattice models in the same dimension [see Sec. III E and Fig. 1(a)] and is called anomalous if otherwise [see Sec. IIIF and Fig. 1(b)] [3,25]. An $(n-1)$ d domain wall between two anomaly-free $n$ d topological orders is called anomaly-free if it can be realized by a $(n-1) \mathrm{d}$ lattice wall between two $(n+1) \mathrm{d}$ lattice-model realization of two adjacent $(n+1) \mathrm{d}$ topological orders [see Fig. 2(a)] and is called anomalous if otherwise [see Fig. 2(b)]. Anomaly-free/anomalous higher codimensional domain walls can be defined similarly. All potentially anomalous $(n+1) \mathrm{D}$ topological orders form a category $\mathrm{TO}^{n+1}$, in which 1 -morphisms in $\mathrm{TO}^{n+1}$ are defined by potentially anomalous 1-codimensional gapped domain walls and higher morphisms are higher codimensional gapped domain walls. See Ref. [26] for more details. Since objects in $\mathrm{TO}^{n+1}$ are topological orders, we simply use $\mathrm{A}^{n+1}, \mathrm{~B}^{n+1}, \mathrm{C}^{n+1} \in \mathrm{TO}^{n+1}$ to denote $(n+1) \mathrm{D}$ topological orders (see Sec. III A). The superscript ${ }^{n+1}$ represents the spacetime dimension and may be omitted if it is manifest from the context. We denote the trivial $(n+1) \mathrm{D}$ topological order by $\mathrm{I}^{n+1}$ (see Sec. III D) and denote the stacking of two $(n+1) \mathrm{D}$ topological orders $\mathrm{A}^{n+1}$ and $\mathrm{B}^{n+1}$ by $\mathrm{A}^{n+1} \otimes \mathrm{B}^{n+1}$. The data $\mathrm{I}^{n+1}$ and $\otimes$ endow the $(n+1)$-category $\mathrm{TO}^{n+1}$ with a structure of a symmetric monoidal $(n+1)$-category. All $(n+1) \mathrm{D}$ anomaly-free topological orders, together with all anomaly-free domain walls of all codimensions, form a symmetric monoidal $(n+1)$-category of anomaly-free $(n+1) \mathrm{D}$ topological orders, denoted by $\mathrm{TO}_{\mathrm{af}}^{n+1}$ (see Sec. III E and Ref. [26] for more details).

We recall a few notions introduced in Ref. [26]. We denote the trivial $(n-1) \mathrm{D}$ domain wall between $\mathrm{A}^{n}$ and $\mathrm{A}^{n}$ by $\mathrm{id}_{\mathrm{A}}$, and the trivial $(n-2) \mathrm{D}$ domain wall between $\mathrm{id}_{\mathrm{A}}$ and $\mathrm{id}_{\mathrm{A}}$ by $\mathrm{id}_{\mathrm{A}}^{2}$, and so on and so forth.

As objects in a higher category, the notion of "same topological order" is nontrivial. Physically, two anomaly-free topological orders, $\mathrm{M}$ and $\mathrm{M}^{\prime}$, are equivalent if they can deform into each other smoothly without closing the energy gap (i.e., without phase transition), i.e., via a continuous path. However, there are different paths that correspond to different ways that $\mathrm{M}$ and $\mathrm{M}^{\prime}$ are equivalent. Those different classes of paths are described by $\pi_{1}\left(\mathfrak{M}^{n+1}\right)$.

Such a deformation corresponds to an invertible domain wall (which is always gapped; see Definition 21) between the two topological orders.

Definition 1. Two anomaly-free topological orders $M$ and $\mathrm{M}^{\prime}$ are called equivalent if they can be connected by an invertible domain wall $\hat{\gamma}$. We denote this isomorphism by $\mathrm{M} \stackrel{\hat{\gamma}}{\simeq} \mathbf{M}^{\prime}$ or $\hat{\gamma}: \mathrm{M} \simeq \mathrm{M}^{\prime}$. The invertible domain walls are classified by $\pi_{1}\left(\mathfrak{M}^{n+1}\right)$.

The objects in $\mathrm{TO}_{\mathrm{af}}^{n+1}$ are actually the equivalent classes of topological orders, under the above equivalent relations (which correspond to isomorphisms in category). When we say two topological orders are the "same," they can be equivalent in many different ways, described by different invertible domain walls.

\section{B. Excitations in a topological order}

In an $n d$ potentially anomalous topological order $\mathrm{C}^{n+1} \in$ $\mathrm{TO}^{n+1}$ [i.e., in $(n+1)$-dimensional spacetime], the point-like (0d), string-like $(1 \mathrm{~d}), \ldots,(n-1) \mathrm{d}$ excitations form a fusion $n$-category, which is denoted as $\operatorname{Hom}\left(\mathrm{C}^{n+1}, \mathrm{C}^{n+1}\right)$, or simply $\mathcal{C}^{n}$ (see Definition 17). By abusing the notation, we set

$$
\Omega \mathrm{C}^{n+1}:=\operatorname{Hom}\left(\mathrm{C}^{n+1}, \mathrm{C}^{n+1}\right)=\mathcal{C}^{n} .
$$

We set the convention of the superscript: $\Omega \mathrm{C}^{n+1}=\Omega\left(\mathrm{C}^{n+1}\right)$. Excitations of codimension-1 can be fused but not braided. If we exclude the 1-codimensional excitations, we obtain a braided fusion $(n-1)$-category, which is precisely the looping $\Omega \mathcal{C}^{n}$ of $\mathcal{C}^{n}$ (see Sec. III H). The fusion $n$-category $\mathcal{C}^{n}$ does not carry the full information about the $n$ d topological order, since $\mathcal{C}^{n}$ only describes the excitations within the $n \mathrm{~d}$ topological order. There are different topological orders (that differ by stacking invertible topological orders [25,41,42]) which have identical excitations. To fully describe an $n d$ topological order $\mathrm{C}^{n+1}$, we need not only the information about the excitations $\mathcal{C}^{n}$, but also the additional information on invertible topological orders. We can also say that $n$ d potentially anomalous topological orders (without any symmetry) are classified, up to invertible topological orders, by fusion $n$-categories [25,26,30,31] (see Propositions 17 and 21).

Similar to topological order, it is tricky to determine if two fusion higher categories are the same or not. In general, we can only say whether the two fusion higher categories are equivalent or not.

Definition 2. Two fusion higher categories, $\mathcal{M}$ and $\mathcal{M}^{\prime}$ are equivalent if there exist a functor $F: \mathcal{M} \rightarrow \mathcal{M}^{\prime}$ and $G$ : $\mathcal{M}^{\prime} \rightarrow \mathcal{M}$ such that $F \circ G \simeq \operatorname{id}_{\mathcal{M}}$ and $G \circ F \simeq \operatorname{id}_{\mathcal{M}^{\prime}}$, where $\simeq$ are natural isomorphisms. Such an equivalence $F$ is denoted by $\mathcal{M} \stackrel{F}{\simeq} \mathcal{M}^{\prime}$ or $F: \mathcal{M} \simeq \mathcal{M}^{\prime}$.

Here, we like to clarify that for simplicity we use the terms of functor, natural isomorphism, algebra object, etc., while they should all be understood as higher categorifications in higher categories.

\section{Holographic principle for topological order}

It was pointed out in Ref. [25] that a potentially anomalous $(n+1) \mathrm{D}$ topological order $\mathrm{C}^{n+1}$ uniquely determines an anomaly-free topological order $\mathrm{M}^{n+2}$ in one-higher dimension where $\mathrm{C}^{n+1}$ can be viewed as a boundary of $\mathrm{M}^{n+2}$ [see Fig. 1(b)]. This boundary-bulk relation is the holographic principle of topological order: "anomaly" = "topological order in one-higher dimension" [3,25-27]. Such a point of view on anomaly is quite different from viewing anomaly as a noninvariance of path-integral measure, and the anomalies under the new point of view are in general noninvertible (since the topological orders are in general noninvertible). We denote this relation between two topological orders by

$$
\mathrm{M}^{n+2}=\operatorname{Bulk}\left(\mathrm{C}^{n+1}\right)
$$

(see Proposition 16). Since $\mathrm{M}^{n+2}$ is anomaly-free, its bulk is trivial, i.e.

$$
\operatorname{Bulk}\left(\mathrm{M}^{n+2}\right)=\operatorname{Bulk}^{2}\left(\mathrm{C}^{n+1}\right)=\mathrm{I}^{n+3} .
$$

In other words, Bulk is a "categorified" differential. 
In fact, we have a stronger version of the holographic principle [see (43) and Fig. 4(b)]:

The excitations in the topological order $\mathrm{C}^{n+1}$, described by the fusion $n$-category $\Omega \mathrm{C}^{n+1}$, can already uniquely determine the bulk anomaly-free topological order $\mathrm{M}^{n+2}$. We denote the map from fusion higher categories $\Omega \mathrm{C}^{n+1}$ to topological orders $\mathrm{M}^{n+2}$ as

$$
\operatorname{bulk}\left(\Omega \mathrm{C}^{n+1}\right)=\mathrm{M}^{n+2} \text {. }
$$

The bulk topological order in turn determines a fusion $n$-category $\Omega \mathrm{M}^{n+2}$ describing excitations in $\mathrm{M}^{n+2}$. After dropping all 1-codimensional excitations, we obtain a braided fusion ( $n-1)$-category $\Omega^{2} \mathrm{M}^{n+2}$. For simplicity, throughout this work, we also use the following convention, for example,

$$
\begin{aligned}
\mathcal{C}^{n} & :=\Omega \mathrm{C}^{n+1}, & \mathcal{C}^{n-1}:=\Omega \mathcal{C}^{n}=\Omega^{2} \mathrm{C}^{n+1} ; \\
\mathcal{M}^{n+1} & :=\Omega \mathrm{M}^{n+2}, & \mathcal{M}^{n}:=\Omega \mathcal{M}^{n+1}=\Omega^{2} \mathrm{M}^{n+2} .
\end{aligned}
$$

The boundary-bulk relation bulk $\left(\Omega \mathrm{C}^{n+1}\right)=\operatorname{bulk}\left(\mathcal{C}^{n}\right)=\mathrm{M}^{n+2}$ reduces to the main results in Refs. [26,27] (see Sec. III I)

$$
\mathcal{M}^{n}=Z_{1}\left(\mathcal{C}^{n}\right) \text { or } \quad \Omega^{2} \mathrm{M}^{n+2}=Z_{1}\left(\Omega \mathrm{C}^{n+1}\right),
$$

where $Z_{1}$ is the monoidal center (or $E_{1}$-center, or Drinfeld center for fusion 1-categories). For a more detailed description of topological orders in arbitrary dimensions, see Sec. III and Refs. [25-31].

\section{Algebraic higher symmetry}

Now, we are ready to describe algebraic higher symmetry. First, let us describe a very general view of symmetry.

Definition ${ }^{\text {ph }} 3$. A symmetry is simply a way to select a set of local operators $\{O\}$, called symmetric local operators, that form a linear vector space:

$$
O_{1}+O_{2} \in\{O\}, \quad \forall O_{1}, O_{2} \in\{O\},
$$

and form a linear algebra

$$
O_{1} O_{2} \in\{O\}, \forall O_{1}, O_{2} \in\{O\} .
$$

The symmetric Hamiltonians are simply sums of those selected local operators.

The standard way to select the symmetric local operators is via symmetry transformations that form a group $G$,

$$
\left\{O_{G} \mid W_{g} O_{G}=O_{G} W_{g}, g \in G\right\},
$$

where the symmetry transformation $W_{g}$ acts on the whole space. The Hamiltonians formed by the sums of local operators in $\left\{O_{G}\right\}$ is said to have a 0 -symmetry $G$.

For a 0 -symmetry given by a group $G$ in spatial $n$ dimension, if the ground state of a symmetric Hamiltonian is a symmetric product state, then point-like excitations are described by the representations of $G$, which are called charged particles. We denote the category of these representations by $1 \mathcal{R}$ ep $G \equiv \mathcal{R}$ ep $G$. These excitations can be fused and braided, and can be condensed to form higher dimensional excitations, called condensation descendants. All these excitations form a (symmetric) fusion $n$-category, denoted by $n \mathcal{R} \operatorname{ep} G$. Due to the Tannaka duality [43] between $\operatorname{Rep}(G)$ and $G$, the fusion and braiding properties (i.e., the conservation law) of the pointlike excitations can fully determine the symmetry group $G$. When $n=2$, we believe that the constructed $n \mathcal{R} \operatorname{ep} G$ outlined above is the same as that in Ref. [44].

In fact, fusion $n$-category $n \mathcal{R e p} G$ can also determine a set of local operators in $n$-dimensional space, denoted as $\left\{O_{n \mathcal{R} \text { ep } G}\right\}$. The set $\left\{O_{n \mathcal{R e p} G}\right\}$ describes all possible local interactions among the excitations described by $n \mathcal{R} \operatorname{ep} G$ that preserve all the fusion and braiding properties of the excitations. For example, $\left\{O_{n \mathcal{R} \operatorname{ep} G}\right\}$ contain all the operators that create particle-antiparticle pairs. It also contain all the operators that create a small loop of string-like excitations, small ball of membrane-like excitations, etc. There are also potential interactions between those excitations. We believe all those operators generate the whole set $\left\{O_{n \mathcal{R e p} G}\right\}$. However, $\left\{O_{n \mathcal{R e p} G}\right\}$ does not contain operators that create a single particle that carries nontrivial representation (i.e., single charged particle). Such operators will break the symmetry.

Above, we have described two ways (i.e., two symmetries) which select two sets of local operators, $\left\{O_{G}\right\}$ and $\left\{O_{n \mathcal{R} \text { ep } G}\right\}$. We believe that

there is one-to-one correspondence between the local operators in the two sets, $\left\{O_{G}\right\}$ and $\left\{O_{n \mathcal{R} \text { ep } G}\right\}$, such that the two corresponding local operators share the same properties (such as identical operator algebra relations). In other words, the linear algebras formed by $\left\{O_{G}\right\}$ and $\left\{O_{n \mathcal{R e p} G}\right\}$ are isomorphic.

Definition $^{\text {ph }}$ 4. Consider two symmetries (i.e., two ways) that select two sets of local operators $\{O\}$ and $\left\{O^{\prime}\right\}$. The two symmetries are said to be holographically equivalent (holoequivalent) if the linear algebras formed by $\{O\}$ and $\left\{O^{\prime}\right\}$ are isomorphic.

The reason we use the term holographically is due to the Propositions 1 and 2. Note that two holo-equivalent symmetries may be generated by transformations that are not related by a unitary transformation, so "holo-equivalent" is more general then "equivalent" for symmetries.

Thus, the symmetry described by the transformations $G$ and the symmetry described by the fusion $n$-category $n \mathcal{R}$ ep $G$ are holo-equivalent. This correspondence represents a categorical view of symmetry, which is heavily used in Refs. [45,46].

The 0-symmetry transformations $W_{g}$ that acts on the whole space can be generalized so that the generalized symmetry transformations $W_{i}$ can act on any loops, any closed membranes, etc. We call the new symmetry algebraic higher symmetry, which can be beyond higher groups. The algebraic higher symmetry described by the transformations $W_{\alpha}$ select a set of local operators

$$
\left\{O_{W} \mid W_{\alpha} O_{W}=O_{W} W_{\alpha}\right\},
$$

where $\alpha$ labels different symmetry transformations. The label $\alpha$ may include various closed subspaces of the space manifold, 
where the symmetry acts. In Secs. IV and V, we discuss some examples of algebraic higher symmetries via the symmetry transformations $W_{\alpha}$. But a mathematical definition (i.e., a macroscopic definition not involving lattice) of algebraic higher symmetries in terms of symmetry transformations $W_{\alpha}$ is not easy to formulate.

In the following, we will use the categorical view of symmetry to obtain a mathematical definition of algebraic higher symmetry. First, we have a mathematical definition of anomaly-free property of algebraic higher symmetry:

Definition $^{p h} 5$. An $n \mathrm{~d}$ algebraic higher symmetry is anomaly-free if there exists a symmetric gapped Hamiltonian in the same dimension whose ground state is a nondegenerate product state. In other words, the gapped ground state is nondegenerate for any closed space manifolds. Such nondegenerate ground state is called a trivial symmetric state. The excitations on top of such a ground state are called charge objects, which carry "representations" of the algebraic higher symmetry.

We note that the excitations (the charge objects) may be point-like, string-like, membrane-like, etc. In particular, for an algebraic $k$-symmetry that acts on closed subspace of codimension $k$, its charge objects has dimension $k$.

Motivated by the Tannaka duality of 0 -symmetry described by a group, we propose that an anomaly-free algebraic higher symmetry in $n$ d boson systems is completely characterized by the excitations on top of its trivial symmetric state. In this paper, we use this property to define algebraic higher symmetry.

Those excitations on a trivial symmetric state form a very special fusion $n$-category $\mathcal{R}$ (called the representation category of the symmetry). To see in which way the fusion $n$-category is special, we note that the symmetry described by $\mathcal{R}$ can be explicitly broken. This explicit symmetry-breaking process will change $\mathcal{R}$ to another fusion $n$-category $n \mathcal{V e c}$, where $n \mathcal{V}$ ec describes point-like, string-like, etc., excitations in a product state without any symmetry (see Sec. IIIH), so $\mathcal{R}$ is a special fusion $n$-category that is equipped with a top-faithful monoidal functor $\mathcal{R} \stackrel{\beta}{\rightarrow} n \mathcal{V}$ ec, where the functor $\beta$ describes the explicit symmetry-breaking process. Such a fusion $n$-category $\mathcal{R}$ is said to be local.

The anomaly-free bosonic algebraic higher symmetries are classified by local fusion $n$-categories $\mathcal{R}$, i.e., by the data $\mathcal{R} \stackrel{\beta}{\rightarrow} n \mathcal{V e c}$ [see Fig. 3(a) and Sec. VIB].

We can use this classification as a formal definition of algebraic higher symmetry. For simplicity, in this paper, we usually drop $\beta$ and use the representation category $\mathcal{R}$ to describe an algebraic higher symmetry. For example, a finite 0 -symmetry $G$ in $n$-dimensional space has a representation category $n \mathcal{R} \operatorname{ep} G$ and can also be referred as a $n \mathcal{R} \operatorname{ep} G$ symmetry.

As a symmetry, the algebraic higher symmetry characterized by $\mathcal{R}$ also selects a set of symmetric local operators $\left\{O_{\mathcal{R}}\right\}$, which describe all possible local interactions between excitations described by $\mathcal{R}$. If the set of local operators selected by the transformations $W_{\alpha}$ [see (13)] has a one-to-one correspondence with the set of local operators selected by

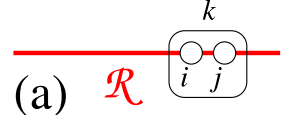

FIG. 3. (a) An algebraic higher symmetry in $n \mathrm{~d}$ bosonic systems is fully characterized by its charge objects (the excitations in trivial symmetric state), which form a local fusion $n$-category $\mathcal{R}$. The symmetry selects a set of local operators $\left\{O_{\mathcal{R}}\right\}$ which are said to have the algebraic higher symmetry $\mathcal{R}$. (b) A categorical symmetry for bosonic systems in $n$-dimensional space is characterized by an anomaly-free topological order $M$ in one higher dimension. The categorical symmetry $\mathrm{M}$ also selects a set of local operators, which is given by all the boundary local interactions, $\left\{O_{\mathrm{M}}\right\}$, of the bulk topological order M. An algebraic higher symmetry $\mathcal{R}$ is holo-equivalent to a categorical symmetry given by $\mathrm{M}=\operatorname{bulk}(\mathcal{R})$. The holo-equivalence means that the algebraic higher symmetry $\mathcal{R}$ and the categorical symmetry $\mathrm{M}=\operatorname{bulk}(\mathcal{R})$ select equivalent sets of local operators, i.e., there is one-to-one correspondence between $\left\{O_{\mathcal{R}}\right\}$ and $\left\{O_{\mathrm{M}}\right\}$, such that the two corresponding local operators have the same operator algebra relations. In this sense, the systems with an algebraic higher symmetry $\mathcal{R}$ also have the categorical symmetry $\mathrm{M}=\operatorname{bulk}(\mathcal{R})$.

the local fusion $n$-category $\mathcal{R}$, i.e., if $\left\{O_{W}\right\} \simeq\left\{O_{\mathcal{R}}\right\}$, then $\mathcal{R}$ describes the algebraic higher symmetry defined by the transformations $W_{\alpha}$.

It is possible that two local fusion $n$-categories, $\mathcal{R}$ and $\mathcal{R}^{\prime}$, select the equivalent local operator algebras.

Definition $^{\text {ph }} 6$. If $\left\{O_{\mathcal{R}}\right\}$ and $\left\{O_{\mathcal{R}^{\prime}}\right\}$ form isomorphic linear algebras (i.e., there is a one-to-one correspondence between $\left\{O_{\mathcal{R}}\right\}$ and $\left\{O_{\mathcal{R}^{\prime}}\right\}$ such that the corresponding operators have the same operator algebra relations), then the two symmetries are called holo-equivalent.

Later we will show that $n \mathcal{R} \operatorname{ep} G$ and $n \mathcal{V e c}_{G}$ are both local fusion $n$-categories if $G$ is a finite group. Their corresponding algebraic higher symmetries are holo-equivalent.

In this paper, we mainly discuss anomaly-free algebraic higher symmetry. For simplicity, by an algebraic higher symmetry we mean an anomaly-free algebraic higher symmetry unless indicated otherwise.

We further generalize the notion of algebraic higher symmetry, by introducing a notion of $\mathcal{V}$-local fusion $n$-category (see Definition 28), which has a top-faithful surjective monoidal functor $\mathcal{R} \stackrel{\beta}{\rightarrow} \mathcal{V}$, where $\mathcal{V}$ is a fusion $n$-category. When $\mathcal{V}=n \mathcal{V}$ ec, $\mathcal{R}$ describes algebraic higher symmetry in $n$ d bosonic systems. When $\mathcal{V}=n \mathrm{~s} \mathcal{V}$ ec, where $n \mathrm{~s} \mathcal{V e c}$ is the fusion $n$-category of super $n$-vector spaces, $\mathcal{R}$ describes algebraic higher symmetry in $n$ d fermionic systems.

The anomaly-free fermionic algebraic higher symmetries are classified by the data $\mathcal{R} \stackrel{\beta}{\rightarrow} n \mathrm{~s} \mathcal{V}$ ec, where $\mathcal{R}$ is a fusion $n$-category.

For some discussions on fermionic topological orders (with $\mathcal{R}=n$ s Vec), see Refs. [47-49], and on fermionic SPT/SET orders (with $\mathcal{R} \stackrel{\beta}{\rightarrow} n$ s $\mathcal{V e c}$ ) see Refs. [46,50-58]. 
More general choices of $\mathcal{V}$ can describe systems formed by anyons or other higher dimensional topological excitations, so the notion of a generalized algebraic higher symmetry allows us to study the symmetry of bosonic and fermionic systems at equal footing. It is interesting to see that the boson, fermion, and anyon statistics can be encoded in a generalization of algebraic higher symmetry.

\section{E. Dual symmetry}

An algebraic higher symmetry can be understood via a more general notion: categorical symmetry. Before explaining categorical symmetry, let us explain a simpler notion of dual symmetry. It was pointed out in Ref. [19] that an $n$ d system with 0 -symmetry $G$ also has a dual algebraic $(n-1)$ symmetry denoted by $\widetilde{G}^{(n-1)}$.

We may use the holographic view to understand the appearance of the dual symmetry. We note that the symmetric sub-Hilbert space of a $G$-symmetric system in $n$-dimensional space can be viewed as a boundary of a one-higherdimensional $G$-gauge theory $[19,59]$ denoted by $\mathrm{GT}_{G}^{n+2}$. The fusion (the conservation) of the bulk point-like gauge charges in the $G$-gauge theory gives rise to the 0 -symmetry $G$. The bulk $\mathrm{GT}_{G}^{n+2}$ also has $(n-1)$ d gauge flux. The fusion (the conservation) of the bulk gauge flux in the $G$-gauge theory gives rise to the algebraic $(n-1)$-symmetry $\widetilde{G}^{(n-1)}$ (see Sec. IV). We stress that both the 0 -symmetry $G$ and the dual algebraic $(n-1)$-symmetry $\widetilde{G}^{(n-1)}$ are present at all the boundaries if we view the boundaries as lattice boundary Hamiltonians or lattice boundary conditions [19] (for details, see the next subsection). However, for a gapped boundary, viewed as a quantum ground state, one of the 0 -symmetry and algebraic $(n-1)$-symmetry, or some of their combinations must be spontaneously broken $[19,60]$.

If we condense all gauge flux, we obtain a boundary with the 0 -symmetry $G$ and the spontaneously broken algebraic $(n-1)$-symmetry $\widetilde{G}^{(n-1)}$. The boundary excitations are described by $n \mathcal{R}$ ep $G$. This boundary corresponds to the usual $G$-symmetric product state whose excitations are also described by $n \mathcal{R} \operatorname{ep} G$.

If we condensed all gauge charges, we obtain a boundary with the dual algebraic $(n-1)$-symmetry $\widetilde{G}^{(n-1)}$ and the spontaneously broken 0 -symmetry $G$. The boundary excitations are described by a local fusion $n$-category $n \mathcal{V} \mathrm{ec}_{G}$. This is the usual spontaneous $G$-symmetry breaking state. The nontrivial fusion (the conservation) of the symmetry-breaking domain walls is also described by $n \mathcal{V} \mathrm{ec}_{G}$, which gives rise to the dual algebraic $(n-1)$-symmetry $\widetilde{G}^{(n-1)}$. Thus, the dual symmetry $\widetilde{G}^{(n-1)}$ can also be represented by its representations category, which is just the fusion $n$-category, $n \mathcal{V} \mathrm{ec}_{G}$, of $G$-graded vector spaces. For such a boundary, the dual algebraic $(n-1)$ symmetry $\widetilde{G}^{(n-1)}$ is not spontaneously broken.

If the boundary Hamiltonians have both the 0-symmetry $G$ and the dual algebraic $(n-1)$-symmetry $\widetilde{G}^{(n-1)}$, we should see a boundary phase where both the 0 -symmetry $G$ and the dual algebraic $(n-1)$-symmetry $\widetilde{G}^{(n-1)}$ are not spontaneously broken. Indeed, such a boundary phase does exist, and it must be gapless. This is because to get a gapped boundary, we must condense enough bulk excitations at the boundary, which break one of the 0 -symmetry and algebraic $(n-1)$-symmetry, or some of their combinations. If we do not condense any bulk excitations, the boundary can only be gapless [61,62].

We see that it is better to view a system with $G$-symmetry as a boundary of the $G$-gauge theory in one higher dimension. This holographic point of view allows us to see the accompanying dual symmetry (i.e., the algebraic $(n-1)$-symmetry $\widetilde{G}^{(n-1)}$ ) clearly. Using a categorical language, the point-like excitations carrying group representations (the charge objects) in an $n$ d $G$-symmetric product state generate a local fusion $n$-category $n \mathcal{R} \operatorname{ep} G$. The same local fusion $n$-category $n \mathcal{R} \operatorname{ep} G$ also describes the excitations on a boundary of $G$-gauge theory $\mathrm{GT}_{G}^{n+2}$, i.e., $\mathrm{GT}_{G}^{n+2}=\operatorname{bulk}(n \mathcal{R} \operatorname{ep} G)$ (see Sec. III I). This links the 0 -symmetry $G$ to the $G$-gauge theory $\mathrm{GT}_{G}^{n+2}$ in one higher dimension. The boundary with excitations $n \mathcal{R} \operatorname{ep} G$ can be obtained from $\mathrm{GT}_{G}^{n+2}$ by condensing the gauge flux.

$\mathrm{GT}_{G}^{n+2}$ has another boundary whose excitations are described by another fusion $n$-category $n \mathcal{V} \mathrm{ec}_{G}$. This boundary is obtained by condensing gauge charges. In this case, the gaugeflux excitations are not condensed, and their nontrivial fusion gives rise to the dual algebraic $(n-1)$-symmetry $\widetilde{G}^{(n-1)}$. In fact, $n \mathcal{V} \mathrm{ec}_{G}$ is the representation category that describes the charge objects of the dual symmetry $\widetilde{G}^{(n-1)}$. In summary, we have

$$
\mathrm{GT}_{G}^{n+2}=\operatorname{bulk}\left(n \mathcal{V} \operatorname{ec}_{G}\right)=\operatorname{bulk}(n \mathcal{R e p} G) .
$$

We see that both 0 -symmetry $G$ and its dual algebraic $(n-1)$-symmetry $\widetilde{G}^{(n-1)}$ share the same $G$-gauge theory $\mathrm{GT}_{G}^{n+2}$ in one higher dimension. Thus, we can view $\mathrm{GT}_{G}^{n+2}$ as a combined symmetry, denoted by $G \vee \widetilde{G}^{(n-1)}$. The combined symmetry is referred as categorical symmetry. It is in this sense that we say that the categorical symmetry $G \vee \widetilde{G}_{\widetilde{G}^{(n-1)}}$ is bigger then the symmetry $G$ and the dual symmetry $\widetilde{G}^{(n-1)}$. We like to mention that the combined symmetry is similar to the "materialized symmetry" in Ref. [5]. However, there is a difference: The categorical symmetry $G \vee \widetilde{G}^{(n-1)}$ is a symmetry on $n$ d boundary, while the materialized symmetry is for $(n+1) \mathrm{d}$ bulk.

It is possible to realize the above model-independent discussion by concrete lattice models. We expect that the Levin-Wen type of lattice models can be generalized to higher dimensions. Similar to the $2+1 \mathrm{D}$ case [34,59], an $n+2 \mathrm{D}$ model is built on a chosen fusion $n$-category $\mathcal{C}$ and a gapped boundary is built on a chosen $\mathcal{C}$-module. Then the $G$-gauge theory $\mathrm{GT}_{G}^{n+2}$ can be realized by such a lattice model by choosing $\mathcal{C}=n \mathcal{R}$ ep $G$. One of its gapped boundary $n \mathcal{R} \operatorname{ep} G$ can be realized by the boundary lattice model built on the obvious $n \mathcal{R}$ ep $G$-module $n \mathcal{R}$ ep $G$. The other gapped boundary $n \mathcal{V} c_{G}$ can be realized by the boundary lattice model built on the $n \mathcal{R} \operatorname{ep} G$-module $n \mathcal{V}$ ec, where the module structure on $n \mathcal{V e c}$ is induced from the fiber functor $n \mathcal{R} \operatorname{ep} G \rightarrow n \mathcal{V e c}$, and $n \mathcal{V e c}_{G}$ is the category of $n \mathcal{R e p} G$-module endo-functors on $n \mathcal{V}$ ec. Mathematically, it is just a manifestation of Morita equivalence between $n \mathcal{R} \operatorname{ep} G$ and $n \mathcal{V} \operatorname{ec}_{G}$.

We would like to mention that a structure similar to categorical symmetry was found previously in anti-de Sitter/ conformal field theory (AdS/CFT) correspondence [63-65], where a global symmetry $G$ at the high-energy boundary is related to a gauge theory of group $G$ in the low-energy bulk. In this paper, we stress that the categorical symmetry encoded 
by the bulk $G$-gauge theory not only contains the $G$ symmetry at the boundary but also contains a dual algebraic higher symmetry $\widetilde{G}^{(n-1)}$ at the boundary. We developed a categorical theory for this holographic point of view for both 0 -symmetry and algebraic higher symmetry. This allows us to gauge the algebraic higher symmetry, classify the anomalies for a given algebraic higher symmetry, identify which algebraic higher symmetries are holo-equivalent, identify duality relations for low-energy effective theories, and classify SET/SPT orders with a given algebraic higher symmetry.

\section{F. Categorical symmetry: A holographic of view of symmetry}

The above is just the simplest example of categorical symmetry. We can generalize the above discussion, and show that, when restricted to the symmetric sub-Hilbert space, an $n$ d system with an algebraic higher symmetry $\mathcal{R}$ can be viewed as a boundary of an anomaly-free topological order $\mathrm{M}=\operatorname{bulk}(\mathcal{R})$ [see (43)]. This allows us to see that our system actually has a categorical symmetry, characterized by topological order $\mathrm{M}$.

Let us first define what is a categorical symmetry. In short,

a categorical symmetry

$$
\begin{aligned}
& =\mathrm{a} \text { noninvertible gravitational anomaly } \\
& =\mathrm{a} \text { topological order in one higher dimension. }
\end{aligned}
$$

To give a more detailed definition, we note that a symmetry is explicitly defined via the algebra of the symmetric local operators that its selects. Let us define categorical symmetry this way.

Definition $^{\text {ph }}$ 7. For an $(n+1)$ d anomaly-free topological order $\mathrm{M}$, the corresponding categorical symmetry is given by the following:

(1) A special boundary of $M$ such that all the excitations in $M$ are either condensed or have nearly zero energy gap. All the bulk excitations have an energy gap larger than a positive fixed value $\Delta_{\text {bulk }}$. Those nearly zero-energy boundary excitations define a low energy boundary Hilbert space.

(2) The symmetric local operators $\left\{O_{\mathrm{M}}\right\}$ are the local operators acting within the low energy boundary Hilbert space.

We note that a bulk topological order $M$ can have many different special boundaries that satisfy the above conditions. We conjecture that different choices of the special boundaries give rise to different sets of symmetric local operators, $\left\{O_{\mathrm{M}}\right\}$ and $\left\{O_{\mathrm{M}}^{\prime}\right\}$, that generate equivalent operator algebra. In other words, $\left\{O_{\mathrm{M}}\right\}$ and $\left\{O_{\mathrm{M}}^{\prime}\right\}$ are holo-equivalent.

Although we define categorical symmetry via a topological order in one higher dimension, in fact, as pointed out in Ref. [19], at least some categorical symmetries can be defined via the patch symmetry transformations without going to one higher dimension, so we believe that the categorical symmetry is really a property of $n \mathrm{~d}$ systems.

For an $n$ d categorical symmetry described by an $(n+1) \mathrm{d}$ topological order $\mathrm{M}$, consider one of its special boundary, such that all the excitations in $\mathrm{M}$ are either condensed or have small but nonzero energy gap on the boundary. Here, small means much smaller than the bulk gap $\Delta_{\text {bulk. }}$. In this case, the special boundary can be viewed as a gapped boundary, whose noncondensing excitations are described by a fusion $n$-category $\mathcal{R}$ that satisfies $\operatorname{bulk}(\mathcal{R})=\mathrm{M}$. The fusion $n$-category $\mathcal{R}$

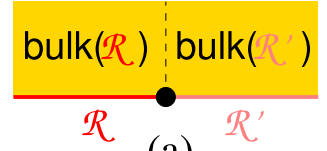

(a)

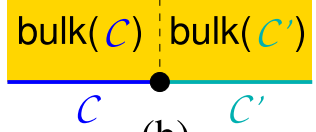

(b)
FIG. 4. (a) Two algebraic higher symmetries $\mathcal{R}$ and $\mathcal{R}^{\prime}$ are holoequivalent if they have the same categorical $\operatorname{symmetry} \operatorname{bulk}(\mathcal{R}) \simeq$ $\operatorname{bulk}\left(\mathcal{R}^{\prime}\right)$. (b) Two sets of low-energy excitations $\mathcal{C}$ and $\mathcal{C}^{\prime}$ are holoequivalent if they have the same categorical symmetry $\operatorname{bulk}(\mathcal{C}) \simeq$ bulk $\left(\mathcal{C}^{\prime}\right)$. Here, holo-equivalent means the states with symmetry $\mathcal{R}$ or $\mathcal{R}^{\prime}$ (or formed by $\mathcal{C}$ or $\mathcal{C}^{\prime}$ ) have a one-to-one correspondence.

defines an algebra higher symmetry which is holo-equivalent to the categorical symmetry $\mathrm{M}$. In other words, $\mathcal{R}$ selects a set of symmetric local operators $\left\{O_{\mathcal{R}}\right\}$ and $\mathrm{M}$ selects a set of symmetric local operators $\left\{O_{\mathrm{M}}\right\}$. The two sets of local operators generate equivalent algebra. We find the following [see Fig. 3(b) and Sec. III I].

Proposition 1. An algebraic higher symmetry $\mathcal{R}$ and a categorical symmetry $\mathrm{M}$ are holo-equivalent, i.e., $\left\{O_{\mathcal{R}}\right\}$ and $\left\{O_{\mathrm{M}}\right\}$ are isomorphic linear alegras, if and only if $\mathrm{M} \simeq \operatorname{bulk}(\mathcal{R})$.

Using the notion of categorical symmetry, we can easily tell when two algebraic higher symmetries are holoequivalent.

Proposition 2. Two algebraic higher symmetries, $\mathcal{R}$ and $\mathcal{R}^{\prime}$, are holo-equivalent if and only if $\operatorname{bulk}(\mathcal{R}) \simeq \operatorname{bulk}\left(\mathcal{R}^{\prime}\right)$ [see Fig. 4(a)].

We note that the dimension- 0 , dimension- 1 , etc., excitations described by $\mathcal{M}$ in the bulk topological order $M$ can be viewed as the dimension- 0 , dimension- 1 , etc., excitations on the boundary if they do not condense. The fusion rule of those bulk excitations corresponds to the conservation law, which leads to the categorical symmetry of the boundary (where the boundary is viewed as a system). However, when the boundary is viewed as a ground state, some of the bulk excitations may condense on the boundary, and the categorical symmetry associated with those condensing excitations are spontaneously broken. So, the boundary, when viewed as a system (i.e., as a Hamiltomian), has the full categorical symmetry, but when viewed as a state, the boundary may spontaneously break part of the categorical symmetry due to condensation of bulk excitations. From this point of view, the categorical symmetry has some special properties $[19,60]$ : For a system with a nontrivial categorical symmetry $M$,

(1) its gapped ground state must spontaneously break the categorical symmetry partially (i.e., some excitations in $\mathcal{M}=$ $\Omega^{2} \mathrm{M}$ condense);

(2) it is impossible to spontaneously break the categorical symmetry completely in a gapped state and possibly not in a gapless state (i.e., it is impossible to condense all excitations in $\mathcal{M})$;

(3) the symmetric ground state with the full categorical symmetry must be gapless (i.e., if none of the excitations in $\mathcal{M}$ condense, the boundary must be gapless).

To see an example of categorical symmetry, Ref. [19] shows that a Hamiltonian on $n$-dimensional lattice with 0 symmetry $\mathbb{Z}_{2}$ also has a $(n-1)$-symmetry $\mathbb{Z}_{2}^{(n-1)}$, so the system actually has a larger $\mathbb{Z}_{2} \vee \mathbb{Z}_{2}^{(n-1)}$ categorical symmetry. 
Such a $\mathbb{Z}_{2} \vee \mathbb{Z}_{2}^{(n-1)}$ categorical symmetry is nothing but the $\mathbb{Z}_{2}$ topological order $\mathrm{GT}_{\mathbb{Z}_{2}}^{n+2}$ (or $\mathbb{Z}_{2}$ gauge theory) in one higher dimension [i.e., in $(n+1)$-dimensional space]. The $\mathbb{Z}_{2}$ symmetry corresponds to the mod- 2 conservation of the point-like $\mathbb{Z}_{2}$ gauge charge. The $\mathbb{Z}_{2}^{(n-1)}(n-1)$-symmetry corresponds to the mod- 2 conservation of the $(n-1)$-dimensional $\mathbb{Z}_{2}$ gauge flux.

The above system can have a gapped phase where $\mathbb{Z}_{2}^{(n-1)}$ is spontaneously broken and $\mathbb{Z}_{2}$ is not broken, which is the usual $\mathbb{Z}_{2}$ symmetric phase [19]. Using the categorical language, we may say that this phase has an (unbroken) algebraic higher symmetry characterized by the local fusion $n$-category $\mathcal{R}=$ $n \mathcal{R}$ ep $\mathbb{Z}_{2}$ (which is nothing but the usual $\mathbb{Z}_{2} 0$-symmetry).

The system can also have a gapped phase where $\mathbb{Z}_{2}$ is spontaneously broken and $\mathbb{Z}_{2}^{(n-1)}$ is not broken, which is the usual $\mathbb{Z}_{2}$-symmetry-broken phase [19]. This phase has an (unbroken) algebraic higher symmetry characterized by the local fusion $n$-category $\mathcal{R}=n \mathcal{V e c}_{\mathbb{Z}_{2}}$, which describes the conservation of symmetry-breaking domain walls.

The quantum critical point of the $\mathbb{Z}_{2}$ symmetry-breaking transition has the full categorical symmetry $\mathbb{Z}_{2} \vee \mathbb{Z}_{2}^{(n-1)}$. In particular, in 1-dimensional space $(n=1)$, the $\mathbb{Z}_{2} \vee \mathbb{Z}_{2}^{(0)}$ categorical symmetry leads to the emergent $\mathbb{Z}_{2} \times \mathbb{Z}_{2}$ symmetry for right movers and left movers [19].

\section{G. Emergence of algebraic higher symmetry and categorical symmetry}

In a practical $n \mathrm{~d}$ condensed matter system, we often have an on-site 0 -symmetry described by a symmetry group $G$. Then the system also has a $G \vee \widetilde{G}^{(n-1)}$ categorical symmetry. However, how can we have a more general higher symmetry or algebraic higher symmetry $\mathcal{R}$, as well as their associated categorical symmetry $\mathrm{M}=\operatorname{bulk}(\mathcal{R})$ in a practical condensed matter system? Certainly, we can try to realize algebraic higher symmetry by fine-tuning. Here, we will describe a situation to have an algebraic higher symmetry without finetuning. In fact, algebraic higher symmetry and categorical symmetry can emerge at low energies.

We will first discuss the emergence of a categorical symmetry M. Once we have an emergent categorical symmetry $M$ (which may or may not be spontaneously broken), then we can determine the emergent algebraic higher symmetry $\mathcal{R}$ (which may or may not be spontaneously broken) by solving the equation $\operatorname{bulk}(\mathcal{R})=\mathrm{M}$. Such a equation may have many solutions for $\mathcal{R}$, but different solutions are all holo-equivalent.

Let us consider a topological order (with or without symmetry) on an $n$ d lattice, whose excitations are described by a fusion $n$-category $\mathcal{C} . \mathcal{C}$ may contain topological excitations not associated with symmetry. $\mathcal{C}$ may also contain charge objects if we have symmetry. Assuming the excitations have a large separation of energy scale, such that all the low-energy excitations (point-like, string-like, etc ) are described a subcategory $\mathcal{C}^{\text {low }}$ of $\mathcal{C}$. All other excitations not in $\mathcal{C}^{\text {low }}$ have large energy gaps, which are assumed to be infinite. Thus, at low energies, we only see the excitations in $\mathcal{C}^{\text {low }}$. Here, we treat all excitations in $\mathcal{C}^{\text {low }}$ on equal footing, and do not distinguish which excitations are charge objects from a symmetry and which excitations are topological excitations. In other words, we pretend all the excitations in $\mathcal{C}^{\text {low }}$ are topological excitations and pretend the system has a (potentially anomalous) topological order without symmetry, whose excitations are described by $\mathcal{C}^{\text {low }}$.

We see that once we know the low-energy excitations $\mathcal{C}^{\text {low }}$ (which may contain possible charge objects from symmetry), the higher energy lattice regularization becomes irrelevant. Thus, we can directly consider a field theory with low-energy excitations $\mathcal{C}^{\text {low }}$. What is the low-energy emergent categorical symmetry? The answer is very simple:

The low-energy effective categorical symmetry for a $n \mathrm{~d}$ field theory with low-energy excitations $\mathcal{C}^{\text {low }}$ is given by a topological order $\mathbf{M}^{\text {low }}=\operatorname{bulk}\left(\mathcal{C}^{\text {low }}\right)$ [see (43)] in one higher dimension.

Here by field theory, we mean a theory whose UV regularization is not specified. When we say a field theory has a property, we mean that there exists a UV regularization of the field theory, such that the regularized theory has the property. It is possible that the same field theory with a different regularization may not have the property. In particular, when we say two field theories are connected by phase transitions, we mean that for any UV regularization of the first field theory, we can find a UV regularization for the second field theory, such that the regularized theories are connected by phase transitions.

The emergent categorical symmetry $M^{\text {low }}$ is very useful (see Sec. VIII):

The categorical symmetry $\mathrm{M}^{\mathrm{low}}$ represents the full information that controls all the low-energy properties of the system.

For example, given a set of low-energy excitations $\mathcal{C}^{\text {low }}$, we like to ask the following questions: When the low-energy excitations condense, what kind new phases are possible? What kind of critical points are possible at the phase transitions? Do we have any principle to address those issues? The answer is the emergent categorical symmetry. This because all the possible low-energy systems (described by all possible interactions of excitations in $\mathcal{C}^{\text {low }}$ ) share the same emergent categorical symmetry $\mathrm{M}^{\text {low }}$. We may view the emergent categorical symmetry $\mathrm{M}^{\text {low }}$ as an "topological invariant" of the low-energy systems. We believe that all other topological invariants of the low-energy systems are contained in the emergent categorical symmetry $\mathrm{M}^{\text {low }}$.

In this paper, we obtain many results assuming exact algebraic higher symmetry and categorical symmetry. Those result remain valid for systems with emergent categorical symmetry $\mathrm{M}^{\text {low }}=\operatorname{bulk}\left(\mathcal{C}^{\text {low }}\right)$. This allows us to apply the results of this paper to some practical situations. In the next subsection, we consider two applications along this line.

\section{H. Categorical symmetry and duality}

A symmetry is useful since it can constrain the properties of a system, such as possible phases and phase transitions, 
the critical properties at the phase transitions, etc. From the above discussion, we see that the constraint from a symmetry actually comes from the corresponding categorical symmetry. This is because the possible physical properties of a system with an algebraic higher symmetry $\mathcal{R}$ are the same as the possible physical properties of a boundary of the topological order $\mathrm{M}=\operatorname{bulk}(\mathcal{R})$ in one higher dimension. In particular, as we have mentioned before, if two symmetries $\mathcal{R}$ and $\mathcal{R}^{\prime}$ have the equivalent categorical symmetry $\operatorname{bulk}(\mathcal{R}) \simeq \operatorname{bulk}\left(\mathcal{R}^{\prime}\right)$, then the two symmetries provide the same constraint on the physical properties [see Figs. 3 and 4(a)], at least within the symmetric sub-Hilbert space. In this case, the two symmetries are holo-equivalent (see Secs. VIE and VIII).

Here, we like state a stronger result:

If two algebraic higher symmetries $\mathcal{R}$ and $\mathcal{R}^{\prime}$ have the equivalent monoidal center $Z_{1}(\mathcal{R}) \simeq Z_{1}\left(\mathcal{R}^{\prime}\right)$, then the two symmetries provide the same constraint on the physical properties, and the two symmetries are holo-equivalent.

In other words, the sets of local operators selected by the two symmetries, $\left\{O_{\mathcal{R}}\right\}$ and $\left\{O_{\mathcal{R}^{\prime}}\right\}$, have a one-to-one correspondence (for example, via a duality transformation; see Ref. [19]) and generate the same algebra. The Hamiltonians as sums of those symmetric local operators also have a oneto-one correspondence, and the corresponding Hamiltonians have the same spectrum.

The above result is motivated by the following consideration: Let $M^{\text {inv }}$ be an invertible topological order in $(n+1)$-dimensional space and $\mathcal{C}_{0}$ be the fusion $n$-category describing the excitations in one gapped boundary of $M^{\text {inv }}$. Then $\mathcal{R}$ and $\mathcal{R}^{\prime} \equiv \mathcal{R} \otimes \mathcal{C}_{0}$ will have the same monoidal center $Z_{1}\left(\mathcal{R}^{\prime}\right)=Z_{1}(\mathcal{R})$, but different bulks: bulk $\left(\mathcal{R}^{\prime}\right)=\mathrm{M} \otimes$ $\mathrm{M}^{\text {inv }} \neq \operatorname{bulk}(\mathcal{R})=\mathrm{M}$. Therefore, requiring $Z_{1}\left(\mathcal{R}^{\prime}\right)=Z_{1}(\mathcal{R})$ does not imply bulk $\left(\mathcal{R}^{\prime}\right) \simeq \operatorname{bulk}(\mathcal{R})$ and does not imply the holo-equivalence. However, if $\mathcal{R}$ is local and describes an algebraic higher symmetry, then $\mathcal{R}^{\prime}=\mathcal{R} \otimes \mathcal{C}_{0}$ is not local and does not describe an algebraic higher symmetry. In other words, the excitations in $\mathcal{C}_{0}$ are topological, which comes from the invertible topological order $\mathrm{M}^{\text {inv }}$ in one higher dimension. Symmetry breaking cannot make them trivial. This is why we think that there is no top-faithful functor $\beta$ that maps $\mathcal{R}^{\prime}=\mathcal{R} \otimes \mathcal{C}_{0}$ into $n \mathcal{V}$ ec. Thus we believe the following:

Proposition 3. If $\mathcal{R}$ and $\mathcal{R}^{\prime}$ are both local (i.e., both describe algebraic higher symmetries), then $Z_{1}\left(\mathcal{R}^{\prime}\right) \simeq Z_{1}(\mathcal{R})$ implies $\operatorname{bulk}\left(\mathcal{R}^{\prime}\right) \simeq \operatorname{bulk}(\mathcal{R})$.

As a result, all possible phases in a system with $\mathcal{R}$ symmetry have a one-to-one correspondence with all possible phases in a system with $\mathcal{R}^{\prime}$ symmetry. In fact, we have a stronger result: All possible states on a system with $\mathcal{R}$ symmetry have a one-to-one correspondence with all possible states on a system with $\mathcal{R}^{\prime}$ symmetry. Those states include gapped states and gapless states. In Ref. [19], some lattice exact duality mappings were discussed for some very simple examples to explicitly demonstrate such a result. This duality relation can be an important application of categorical symmetry.

For example, an $n$ d system with $G 0$-symmetry can be mapped to an $n$ d system with the dual $\widetilde{G}^{(n-1)}(n-1)$ - symmetry, and vice versa. The $G 0$-symmetry and the $\widetilde{G}^{(n-1)}(n-1)$-symmetry are holo-equivalent symmetries. Using the categorical notation, we say the $n \mathcal{R} \operatorname{ep} G$ symmetry and the $n \mathcal{V} \mathrm{ec}_{G}$ symmetry are holo-equivalent symmetries, since $Z_{1}(n \mathcal{R e p} G) \simeq Z_{1}\left(n \mathcal{V} \operatorname{ec}_{G}\right)$.

The above duality result can be generalized even further [see Fig. 4(b) and Sec. VIII]:

Consider two $n$ d field theories with low-energy excitations described by two fusion $n$-categories $\mathcal{C}$ and $\mathcal{C}^{\prime}$ respectively. The two field theories are dual to each other (i.e., are holo-equivalent), if they have equivalent categorical symmetries $\operatorname{bulk}(\mathcal{C}) \simeq \operatorname{bulk}\left(\mathcal{C}^{\prime}\right)$ [see (43)], provided that all other excitations have high energies.

We like to remark that the two field theories may have different symmetries described by different charge objects, forming two different subcategories in $\mathcal{C}$ and $\mathcal{C}^{\prime}$. The two field theories may also have different low-energy topological excitations. In other words, we do not care which excitations are topological excitations and which excitations are charge objects of the symmetries.

When two systems have the same categorical symmetry M, both systems can be simulated by the boundaries of the same bulk topological order $\mathrm{M}$ (since the categorical symmetry is the bulk topological order). Hence, the two systems are holoequivalent. This means that the possible states of the system $\mathcal{C}$ (including condensed states, gapless states, etc.) have a oneto-one correspondence with the possible states of the system $\mathcal{C}^{\prime}$ (see Sec. VIII). Those states are just the possible boundary states of the same $\mathrm{M}$.

\section{Gauging the algebraic higher symmetry and the corresponding $\mathcal{R}$-gauge theory}

Given an $n$ d product state with an on-site 0 -symmetry $G$ (i.e., an anomaly-free 0 -symmetry), we can gauge the symmetry to obtain a state with topological order and no symmetry. The resulting topological order is nothing but the $G$-gauge theory $\mathrm{GT}_{G}^{n+1}$. The excitations in $\mathrm{GT}_{G}^{n+1}$ are described by a fusion $n$-category $\Omega \mathrm{GT}_{G}^{n+1}$. In fact $\Omega \mathrm{GT}_{G}^{n+1}=\Sigma Z_{1}((n-$ 1) $\operatorname{Rep}(G)$ ), where $\Sigma$ is the delooping (see Sec. III H).

Similarly, given an $n$ d product state with an anomaly-free higher symmetry, we can gauge the higher symmetry to obtain a state with topological order and no symmetry. The resulting topological order is described by a higher gauge theory.

Now, given an $n$ d product state with an anomaly-free algebraic higher symmetry $\mathcal{R}$, can we gauge the algebraic higher symmetry to obtain a state with topological order and no symmetry? If we can, then the corresponding topological order is a gauge theory for the algebraic higher symmetry $\mathcal{R}$. We denote such a gauge theory by $\mathrm{GT}_{\mathcal{R}}^{n+1}$, the excitations in which form a fusion $n$-category $\Omega \mathrm{GT}_{\mathcal{R}}$.

In this paper, we propose a way to gauge algebraic higher symmetry, which gives us a construction of $\mathcal{R}$-gauge theory (by constructing the corresponding topological order $\mathrm{GT}_{\mathcal{R}}^{n+1}$ ). Our approach is based on the holographic view of the $\mathcal{R}$ symmetry, which is very different from the usual gauging based on spacetime-dependent symmetry transformations. 


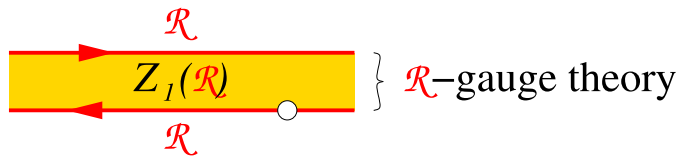

FIG. 5. Gauging the $\mathcal{R}$-symmetry: Stacking two local fusion $n$-category $\mathcal{R}$ over their common bulk $Z_{1}(\mathcal{R})$ gives rise to an fusion $n$-category $\mathcal{R} \underset{Z_{1}(\mathcal{R})}{\otimes} \mathcal{R}^{\text {rev }}$, describing the excitations in $n d$ anomaly-free topological order $\mathrm{GT}_{\mathcal{R}}^{n+1}$, which is the $\mathcal{R}$-gauge theory. The boundary Hamiltonians of $\mathcal{R}$-gauge theory share the same low-energy properties with the Hamiltonians with algebraic higher symmetry $\Omega \mathcal{R}$, if $\mathcal{R}=\Sigma \Omega \mathcal{R}$. We like to remark that $\mathcal{R}$ 's on the two boundaries differ by a parity transformation as indicated by the arrows and superscript ${ }^{\text {rev }}$.

Under the holographic point of view, an algebraic higher symmetry $\mathcal{R}$ gives rise to a 1-higher-dimensional topological order $\mathrm{M}$ such that $\Omega \mathrm{M}=Z_{1}(\mathcal{R})$ [see Fig. 3(b)]. Then the topological order obtained by gauging the $\mathcal{R}$-symmetry in a symmetric product state can be obtained by simply stacking two $\mathcal{R}$ boundaries through their common bulk $Z_{1}(\mathcal{R})$ (see Fig. 5). This is an algebraic way to gauge an symmetry, which work for 0-symmetries, higher symmetries, and algebraic higher symmetries (see Sec. VII C 5).

The excitations in an $n \mathrm{~d} \mathcal{R}$-gauge theory $\mathrm{GT}_{\mathcal{R}}^{n+1}$ are described by an multifusion $n$-category

$$
\Omega \mathrm{GT}_{\mathcal{R}}^{n+1}=Z_{0}(\mathcal{R})=\mathcal{R} \underset{Z_{1}(\mathcal{R})}{\otimes} \mathcal{R}^{\text {rev }},
$$

where $Z_{0}(\mathcal{R}):=\operatorname{Fun}(\mathcal{R}, \mathcal{R})$ is the $E_{0}$-center.

\section{J. Dual of an algebraic higher symmetry}

Using a similar holographic approach, we can also define the dual symmetry $\mathcal{R}$ for an arbitrary algebraic higher symmetry $\mathcal{R}$ as follows: $\mathcal{R}$ and $\widetilde{\mathcal{R}}$ are dual to each other, if they have the same bulk $Z_{1}(\mathcal{R})=Z_{1}(\widetilde{\mathcal{R}})=\mathcal{M}$ and if the stacking of $\mathcal{R}$ and $\widetilde{\mathcal{R}}$ through their bulk gives rise to a trivial topological order (i.e., a product state; see Fig. 6, Definition 30, and Proposition 39):

$$
\mathcal{R} \underset{\mathcal{M}}{\otimes} \widetilde{\mathcal{R}}^{\text {rev }}=n \mathcal{V} \text { ec }
$$

Such a definition reproduces our previous result: The dual of $G 0$-symmetry is the $\tilde{G}^{(n-1)}(n-1)$-symmetry; i.e., $n \mathcal{R} \operatorname{ep}(G)$ and $n \mathcal{V} \mathrm{ec}_{G}$ are dual to each other [19].

We would like to mention that a gapped boundary of $\mathcal{M}$, such as $\mathcal{R}$, is induced by condensing some excitations in $\mathcal{M}$ at the boundary. The collection of those condensing excitations form a so-called condensable algebra $A_{\mathcal{R}}$. The condensable algebra $A_{\mathcal{R}}$ uniquely determines the gapped boundary. A different condensable algebra $A_{\widetilde{\mathcal{R}}}$ unique determines a different gapped boundary $\widetilde{\mathcal{R}}$. Roughly speaking, moving bulk excitation in $\mathcal{M}$ to the $\mathcal{R}$ boundary induces a map from $\mathcal{M}$ to $\mathcal{R}$, the mathematical description of which is a functor $F_{\mathcal{R}}: \mathcal{M} \rightarrow \mathcal{R}$. Under such a map, the condensable algebra $A_{\mathcal{R}}$ is mapped to the trivial excitations in $\mathcal{R}$ (i.e., condensed; see Fig. 6).

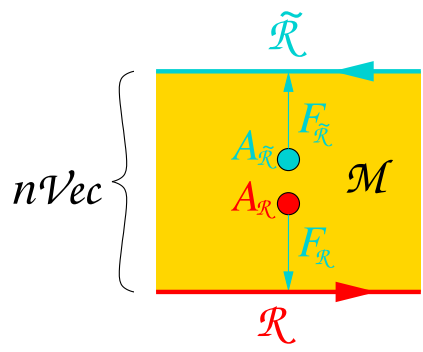

FIG. 6. Stacking $\mathcal{R}$ and $\widetilde{\mathcal{R}}$ through their common bulk gives rise to a trivial product state $\mathcal{R} \otimes \widetilde{\mathcal{R}}^{\text {rev }}=n \mathcal{V}$ ec, if the two algebraic higher symmetries, $\mathcal{R}$ and $\widetilde{\mathcal{R}}$, are dual to each other. Condensing the condensable algebra $A_{\mathcal{R}}\left(\operatorname{resp} . A_{\tilde{\mathcal{R}}}\right)$ produces the $\mathcal{R}$ (resp. $\left.\widetilde{\mathcal{R}}\right)$ boundary. The functor $F_{\mathcal{R}}: \mathcal{M} \rightarrow \mathcal{R}$, induced by moving the bulk excitations to the boundary, maps $A_{\mathcal{R}}$ to the trivial excitation on the $\mathcal{R}$ boundary. Similar for $F_{\tilde{\mathcal{R}}}$.

\section{K. Anomalous algebraic higher symmetry}

Can an algebraic higher symmetry have anomaly? How to describe its anomaly? First, an anomalous symmetry is characterized by two things: symmetry and anomaly. So an anomalous algebraic higher symmetry is characterized by a pair $(\mathcal{R}, \alpha)$, where $\mathcal{R}$ is for symmetry and $\alpha$ for anomaly.

For a 0 -symmetry in $n$-dimensional space, an anomalous symmetry is characterized by a pair $\left(G, \omega_{n+2}\right)$ where $\omega_{n+2} \epsilon$ $H^{n+2}(G, U(1))$ is an $(n+2)$-cocycle. A more physical way to understand the anomalous symmetry $\left(G, \omega_{n+2}\right)$ is to view it as the boundary symmetry of a 1-dimension-higher SPT state [3], which is also characterized by the pair $\left(G, \omega_{n+2}\right)$. We can gauge the $G$-symmetry in the SPT state to get a "twisted" $G$ gauge theory (the Dijkgraaf-Witten theory [66]), denoted by $\mathrm{GT}_{G, \omega_{n+2}}^{n+2}$. In fact, $\mathrm{GT}_{G, \omega_{n+2}}^{n+2}$ is the categorical symmetry that is holo-equivalent to the anomalous symmetry $\left(G, \omega_{n+2}\right)$. Thus, we can also describe the anomalous symmetry $\left(G, \omega_{n+2}\right)$ via its holo-equivalent categorical symmetry $\mathrm{GT}_{G, \omega_{n+2}}^{n+2}$, which is a "twisted" $G$-gauge theory in one higher dimension $[3,19]$. This is the point of view that we will use in this paper.

In fact, under the holographic point of view, a "twisted" $G$ gauge theory in one higher dimension defines an anomalous 0 -symmetry. The boundaries of the "twisted" $G$ gauge theory give rise to all possible phases (including symmetry-breaking phases), as well as all other properties, of systems with the anomalous $G 0$-symmetry.

Similarly, an $n$ d bosonic system with an anomalous higher symmetry described by a higher group $\mathcal{B}\left(G, \pi_{2}, \ldots\right.$ ) (using the notation in Ref. [67]) has a holo-equivalent categorical symmetry characterized by a "twisted" higher gauge theory in one higher dimension. The different anomalies for the higher group $\mathcal{B}\left(G, \pi_{2}, \ldots\right)$ are (partially) characterized by cocycles in $H^{n+2}\left[\mathcal{B}\left(G, \pi_{2}, \ldots\right) ; \mathbb{R} / \mathbb{Z}\right]$. The "twisted" higher gauge theory in one higher dimension defines the anomaly of the anomalous higher symmetry.

The categorical symmetry of an $n$ d Hamiltonian with an anomaly-free algebraic higher symmetry $\mathcal{R}$ is given by $\mathrm{M}=$ $\operatorname{bulk}(\mathcal{R})$. We like to ask whether $\mathrm{M}=\operatorname{bulk}(\mathcal{R})$ describes the $\mathcal{R}$-gauge theory in one higher dimension. The answer is no, simply because $\mathcal{R}$ describes a symmetry in $n \mathrm{~d}$, not in one higher dimension $(n+1) \mathrm{d}$. The gauge theory in one higher 
dimension cannot be a $\mathcal{R}$-gauge theory since $\mathcal{R}$ lives in one lower dimension.

When we discuss $G$-gauge theory in all the dimensions, we have used the fact that the same $G$-symmetry can be defined in all the dimensions. For an algebraic symmetry $\mathcal{R}$ in $n \mathrm{~d}$, what is the corresponding symmetry in one higher dimension $(n+1) \mathrm{d}$ ? This is a highly nontrivial question. It turns out that an algebraic symmetry, in general, cannot be promoted to one higher dimension. Only a special class of algebraic symmetries, described by symmetric local fusion $n$-categories, can be promoted to one higher dimension. This is because a symmetric fusion $n$-category $\mathcal{R}$ in $n \mathrm{~d}$ can be viewed as a braided fusion $n$-category, describing 2-codimensional and higher excitations in one higher dimension [i.e., in $(n+1)$ d]. We then can do a delooping to obtain a fusion $(n+1)$-category $\Sigma \mathcal{R}$ (see Sec. III H). If $\mathcal{R}$ is a symmetric local $n$-category, $\Sigma \mathcal{R}$ is again a symmetric local $(n+1)$-category. So, $\Sigma \mathcal{R}$ describes the $\mathcal{R}$-symmetry in one higher dimension. Since $\Sigma \mathcal{R}$ is also symmetric and local, we can promote further to obtain the corresponding $\mathcal{R}$ symmetry in all higher dimensions $\Sigma^{2} \mathcal{R}$, $\Sigma^{3} \mathcal{R}$, etc. In this subsection, we assume $\mathcal{R}$ to be symmetric local fusion $n$-category.

Now, we can state the nontrivial result:

The categorical symmetry for an anomaly-free algebraic symmetry $\mathcal{R}, \mathrm{M}=\operatorname{bulk}(\mathcal{R})$, is the same as the $\Sigma \mathcal{R}$-gauge theory $\mathrm{GT}_{\Sigma \mathcal{R}}^{n+2}$ in one higher dimension:

$$
\operatorname{bulk}(\mathcal{R})=\mathrm{GT}_{\Sigma \mathcal{R}}^{n+2},
$$

provided that $\mathcal{R}$ is symmetric.

The excitations in the $\Sigma \mathcal{R}$-gauge theory are given by

$$
\Omega \mathrm{GT}_{\Sigma \mathcal{R}}^{n+2}=\Sigma \mathcal{R} \underset{Z_{1}(\Sigma \mathcal{R})}{\otimes} \Sigma \mathcal{R}^{\mathrm{rev}},
$$

which defines the gauging of the algebraic higher symmetry $\Sigma \mathcal{R}$. Equation (19) describes the excitations in $\operatorname{bulk}(\mathcal{R})$ given by $\Omega \operatorname{bulk}(\mathcal{R})=\Sigma Z_{1}(\mathcal{R})$. The fact that $\Sigma Z_{1}(\mathcal{R})=$ $\Sigma \mathcal{R} \underset{Z_{1}(\Sigma \mathcal{R})}{\otimes} \Sigma \mathcal{R}^{\text {rev }}$ follows [30] from the following identity [68]:

$$
\Sigma Z_{1}(\mathcal{R})=Z_{0}(\Sigma \mathcal{R})=\Sigma \mathcal{R} \underset{Z_{1}(\Sigma \mathcal{R})}{\otimes} \Sigma \mathcal{R}^{\text {rev }} .
$$

Similarly, an anomalous algebraic higher symmetry $(\mathcal{R}, \alpha)$ is defined via its categorical symmetry, which corresponds to a twisted $\Sigma \mathcal{R}$-gauge theory in one higher dimension. The twist is produced by an automorphism $\alpha$ of $Z_{1}(\Sigma \mathcal{R})$ (the dashed line in Fig. 7). Such a twisted $\Sigma \mathcal{R}$ gauge theory, denoted by $\mathrm{GT}_{\Sigma \mathcal{R}, \alpha}^{n+2}$, is characterized by its excitations described by (see Fig. 7)

$$
\Omega \mathrm{GT}_{\Sigma \mathcal{R}, \alpha}^{n+2}=\Sigma \mathcal{R} \underset{Z_{1}(\Sigma \mathcal{R})}{\otimes} \alpha \underset{Z_{1}(\Sigma \mathcal{R})}{\otimes} \Sigma \mathcal{R}^{\mathrm{rev}} .
$$

The automorphism $\alpha$ is not arbitrary. It must satisfy $\alpha\left(A_{\widetilde{\Sigma \mathcal{R}}}\right) \simeq A_{\widetilde{\Sigma \mathcal{R}}}$, where $A_{\widetilde{\Sigma \mathcal{R}}}$ is the condensable algebra for the dual symmetry of $\Sigma \mathcal{R}$ (see Sec. VIIC 5). This result allows us to classify types of anomalies that an algebraic higher symmetry can have. Since invertible domain wall

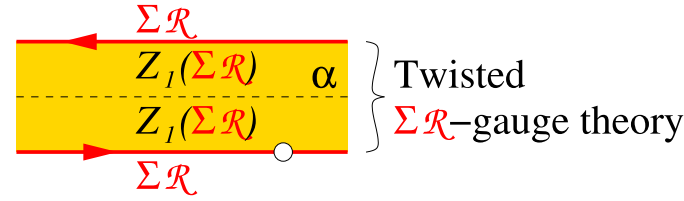

FIG. 7. The topological order described by a twisted $\Sigma \mathcal{R}$ gauge theory has excitations described by $\Sigma \mathcal{R} \underset{Z_{1}(\Sigma \mathcal{R})}{\otimes} \alpha \underset{Z_{1}(\Sigma \mathcal{R})}{\otimes} \Sigma \mathcal{R}^{\text {rev }}$. The twist is done by an automorphism $\alpha$ in $Z_{1}(\Sigma \mathcal{R})$ that keeps the condensable algebraic $A_{\widetilde{\Sigma \mathcal{R}}}$ of the dual symmetry $\widetilde{\Sigma \mathcal{R}}$ unchanged $\alpha\left(A_{\widetilde{\Sigma \mathcal{R}}}\right) \simeq A_{\widetilde{\Sigma \mathcal{R}}}$. The boundary Hamiltonians of twisted $\Sigma \mathcal{R}$-gauge theory correspond to the Hamiltonians with anomalous algebraic higher symmetry $\mathcal{R}$.

include invertible topological orders, the above anomalies include invertible gravitational anomalies, symmetry ('t Hooft) anomalies (which are always invertible), and invertible mixed symmetry-gravitational anomalies.

Anomalous algebraic higher symmetries $\mathcal{R}$ are classified by the automorphisms $\alpha$ of $Z_{1}(\Sigma \mathcal{R})$ such that $\alpha\left(A_{\widetilde{\Sigma \mathcal{R}}}\right) \simeq A_{\widetilde{\Sigma \mathcal{R}}}$. Its categorical symmetry $\mathrm{M}$ satisfies $\Omega \mathrm{M}=\Sigma \mathcal{R} \underset{Z_{1}(\Sigma \mathcal{R})}{\otimes} \alpha \underset{Z_{1}(\Sigma \mathcal{R})}{\otimes} \Sigma \mathcal{R}^{\mathrm{rev}}$.

As an application of the above result, we consider 1d bosonic system with an anomalous $\mathbb{Z}_{2}^{3}$ symmetry. The possible anomalies are classified by $H^{3}\left(\mathbb{Z}_{2}^{3}, U(1)\right)$, which correspond to $2 \mathrm{~d} \mathbb{Z}_{2}^{3}$-SPT orders. The categorical symmetry of the $1 \mathrm{~d}$ anomalous $\mathbb{Z}_{2}^{3}$ symmetry is given by the $2 \mathrm{~d}$ topological order obtained by gauging the corresponding $2 \mathrm{~d} \mathbb{Z}_{2}^{3}$-SPT states. It was found that a particular anomalous $\mathbb{Z}_{2}^{3}$ symmetry, described by a so-called type-III cocycle in $H^{3}\left(\mathbb{Z}_{2}^{3}, U(1)\right)$, has a categorical symmetry described by the $2 \mathrm{~d} D_{4}$ gauge theory $\mathrm{GT}_{D_{4}}^{3}$ $[69,70]$. Certainly, the $1 \mathrm{~d}$ anomaly-free $D_{4}$ symmetry also has a categorical symmetry described by the $2 \mathrm{~d} D_{4}$ gauge theory $\mathrm{GT}_{D_{4}}^{3}$. Therefore, this particular $1 \mathrm{~d}$ anomalous $\mathbb{Z}_{2}^{3}$ symmetry is holo-equivalent to $1 \mathrm{~d} D_{4}$ symmetry. In general, we conjecture the following:

Two anomalous algebraic higher symmetries, $(\mathcal{R}, \alpha)$ and $\left(\mathcal{R}^{\prime}, \alpha^{\prime}\right)$, are holo-equivalent if they satisfy

$$
\begin{aligned}
& \Sigma \mathcal{R} \underset{Z_{1}(\Sigma \mathcal{R})}{\otimes} \alpha \underset{Z_{1}(\Sigma \mathcal{R})}{\otimes} \Sigma \mathcal{R}^{\mathrm{rev}} \\
& \quad \simeq \Sigma \mathcal{R}^{\prime} \underset{Z_{1}\left(\Sigma \mathcal{R}^{\prime}\right)}{\otimes} \alpha^{\prime} \otimes_{Z_{1}\left(\Sigma \mathcal{R}^{\prime}\right)} \Sigma \mathcal{R}^{\prime \mathrm{rev}},
\end{aligned}
$$

which implies that $(\mathcal{R}, \alpha)$ and $\left(\mathcal{R}^{\prime}, \alpha^{\prime}\right)$ have the same categorical symmetry.

One may ask whether a categorical symmetry $M$ can always be viewed as an anomalous algebraic higher symmetry. If the categorical symmetry satisfies $\Omega \mathrm{M}=\Sigma \mathcal{R} \underset{Z_{1}(\Sigma \mathcal{R})}{\otimes}$ $\alpha \underset{Z(\Sigma \mathcal{R})}{\otimes} \Sigma \mathcal{R}^{\text {rev }}$, then the categorical symmetry can indeed be viewed as an anomalous $\mathcal{R}$-symmetry. We believe there exist categorical symmetries that do not have the above form, and 


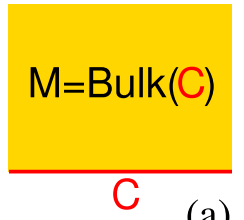

(a)

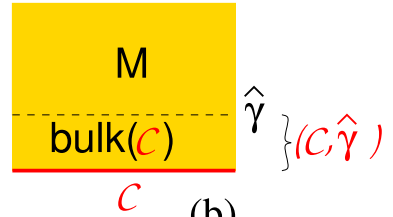

(b)
FIG. 8. (a) Gapped liquid phases in systems with categorical symmetry $M$ are classified by anomalous topological orders $C$ such that $M=\operatorname{Bulk}(C)$. Those gapped liquid phases are partially classified by fusion $n$-categories $\mathcal{C}$ such that $\mathcal{M}=\Omega^{2} \mathrm{M}=Z_{1}(\mathcal{C})$. (b) Gapped liquid phases in systems with categorical symmetry $M$ are classified by the pairs $(\mathcal{C}, \hat{\gamma})$, where $\mathcal{C}$ satisfies bulk $(\mathcal{C})=\mathrm{M}$ and $\hat{\gamma}$ is an invertible domain wall in $\mathrm{M}$.

those categorical symmetries cannot be viewed as an anomalous algebraic higher symmetry. Categorical symmetry play a similar role as anomalous symmetry, but it is more general. In fact, after introducing categorical symmetry, we do not need to use anomalous symmetry any more. The effect of anomalous symmetry can all be covered by categorical symmetry.

\section{Classification of gapped liquid phases for systems with a categorical symmetry}

We can also use the holographic point of view to classify SET/SPT orders with a given algebraic higher symmetry $\mathcal{R}$. But first, let us classify possible gapped liquid phases in $n \mathrm{~d}$ systems with a categorical symmetry $\mathrm{M}$ (assuming $n \geqslant 1$ ). Using boundary-bulk relation, we find the follwing (see Sec. VII A):

For $n$ d lattice systems with a categorical symmetry M, all their gapped liquid phases (which partially break the categorical symmetry spontaneously) are classified by potential anomalous $n$ d topological orders $\mathrm{C}$ (i.e., $n \mathrm{~d}$ boundary topological orders) that satisfy [see Fig. 8(a)

$$
M=\operatorname{Bulk}(C) \text {. }
$$

We note that the categorical symmetry $\mathrm{M}$ is an anomalyfree topological order in one higher dimension.

Since the fusion $n$-categories $\mathcal{C}$ (describing the excitations) partially describes an $n$ d topological order (up to invertible topological orders $[25,41,42]$ and SPT orders), we get a partial classification if we only use $\mathcal{C}$ (see Sec. VII B):

The gapped liquid phases (up to invertible topological orders and SPT orders) in $n$ d lattice systems with categorical symmetry $\mathrm{M}$ are classified by fusion $n$-categories $\mathcal{C}$ that satisfy $\operatorname{bulk}(\mathcal{C})=\mathrm{M}$.

Motivated by the results in Refs. [45,46], we conjecture that, comparing $\mathrm{C}$ and $\mathcal{C}$, the missing information is the invertible topological orders and SPT orders. Thus, we can use a pair $(\mathcal{C}, \hat{\gamma})$ to describe $\mathrm{C}$, where $\hat{\gamma}$ corresponds to invertible topological orders or SPT orders. More precisely [see Fig. 8(b) and Sec. VII B], we find the following:
Gapped liquid phases in $n$ d lattice systems with a fixed categorical symmetry $\mathrm{M}$ are classified by pairs $(\mathcal{C}, \hat{\gamma})$, where $\mathcal{C}$ is a fusion $n$-category that satisfies $\operatorname{bulk}(\mathcal{C})=\mathrm{M}$ and $\hat{\gamma}$ is an invertible domain wall in $\mathrm{M}$.

The invertible domain walls formed by the trivial excitations in the bulk topological order $\mathrm{M}$ are invertible topological orders. The invertible domain walls formed by the topological excitations in the bulk topological order M are SPT orders, since the fusion of the topological excitations encode symmetry. There are also invertible domain walls that exchange excitations. Those invertible domain walls correspond to the automorphisms of the fusion higher category $\Omega \mathrm{M}=\mathcal{M}$ that describes the excitations in the bulk topological order M.

\section{Classification of SET orders and SPT orders with an algebraic higher symmetry}

For systems with a categorical symmetry $M$, in the above, we classify anomaly-free gapped liquid phases $\mathrm{C}$ which partially break the categorical symmetry. Here we assume the unbroken symmetry is an algebraic higher symmetry $\mathcal{R}$, such that $\mathrm{M}=\operatorname{bulk}(\mathcal{R})$. In this case, the classification of gapped liquid phases $\mathrm{C}$ in last subsection includes the classification of anomaly-free gapped liquid phases with a given algebraic higher symmetry $\mathcal{R}$. Which gapped liquid phases do not break the symmetry $\mathcal{R}$ and which spontaneously break the symmetry $\mathcal{R}$ ? To identify the gapped liquid phases $C$ that do not break the symmetry $\mathcal{R}$, first $\mathrm{C}$ should include $\mathcal{R}$ as its excitations, i.e., $\mathcal{R}$ is a subcategory of $\mathcal{C}=\Omega \mathrm{C}$, or more precisely, there is a top fully faithful functor $\iota: \mathcal{R} \hookrightarrow \mathcal{C}$ (see Proposition 27 and Definition 29). Second, $\mathcal{R}$ and $\mathcal{C}$ have the same bulk topological order $\operatorname{bulk}(\mathcal{C})=\operatorname{bulk}(\mathcal{R})$ (i.e., the same categorical symmetry).

This understanding gives us a classification of anomalyfree gapped liquid phases with an anomaly-free algebraic higher symmetry $\mathcal{R}$. First, let us define the notion "anomalyfree gapped liquid phases with an anomaly-free algebraic higher symmetry $\mathcal{R}$ " more carefully. We have defined anomaly-free algebraic higher symmetry in Definition 5. "A phases with a symmetry" means that the symmetry is not spontaneously broken. But how do we determine if an algebraic higher symmetry $\mathcal{R}$ is spontaneously broken or not? There is a macroscopic way to do so (see Sec. VII A):

A gapped phase has a symmetry $\mathcal{R}$ (i.e., $\mathcal{R}$ is not spontaneously broken) if the excitations of phase contain $\mathcal{R}$.

A gapped liquid phase is anomaly-free if it can be realized as the ground of lattice Hamiltonian in the same dimension. Again, there is a way to describe this property macroscopically (see Sec. VII C 1):

If the excitations (described by fusion $n$-category $\mathcal{C}$ ) in the gapped liquid phase with an algebraic higher symmetry $\mathcal{R}$ satisfy bulk $(\mathcal{C}) \simeq \operatorname{bulk}(\mathcal{R})$, then the gapped liquid phase is anomaly-free. 
Here bulk $(\mathcal{C}) \simeq \operatorname{bulk}(\mathcal{R})$ means that the two topological orders bulk $(\mathcal{C})$ and bulk $(\mathcal{R})$ are equivalent, i.e., they can be connected by an invertible (also called transparent) domain wall $\hat{\gamma}$. Note that the invertible domain wall is not unique. Thus, the two topological orders bulk $(\mathcal{C})$ and $\operatorname{bulk}(\mathcal{R})$ can be equivalent in many different ways. We denote a way of equivalence by $\operatorname{bulk}(\mathcal{C}) \stackrel{\hat{\mathcal{Z}}}{\simeq} \operatorname{bulk}(\mathcal{R})$ or $\hat{\gamma}: \operatorname{bulk}(\mathcal{C}) \simeq \operatorname{bulk}(\mathcal{R})$.

Now, we are ready to state some classification results. First, let us describe a simple partial classification:

Given an algebraic higher symmetry described by a local fusion $n$-category $\mathcal{R}$, anomaly-free symmetric gapped liquid phases (up to invertible topological orders and SPT orders) are classified by fusion $n$-categories $\mathcal{C}$ that (1) admit a top fully faithful functor $\iota: \mathcal{R} \hookrightarrow \mathcal{C}$, and (2) satisfy $\operatorname{bulk}(\mathcal{C}) \simeq \operatorname{bulk}(\mathcal{R})($ see Sec. VII A $)$

To get a more complete classification that includes invertible topological orders and SPT orders for the algebraic higher symmetry $\mathcal{R}$, we need to include the equivalence (i.e., the invertible domain wall) $\hat{\gamma}: \operatorname{bulk}(\mathcal{R}) \simeq \operatorname{bulk}(\mathcal{C})$ and use the data $(\mathcal{R} \stackrel{\iota}{\hookrightarrow} \mathcal{C}, \hat{\gamma})$ to classify anomaly-free symmetric gapped liquid phases. However, not every equivalence $\hat{\gamma}$ should be included. We know that the categorical symmetry described by bulk $(\mathcal{R})$ or by bulk $(\mathcal{C})$ includes the algebraic higher symmetry $\mathcal{R}$. The equivalence $\hat{\gamma}$ should not change $\mathcal{R}$ that is contained in bulk $(\mathcal{R})$ and in bulk $(\mathcal{C})$ [68]. For details and the main classification results, see Sec. VII C. Here, we just quote a classification of $\mathcal{R}$-SPT orders, obtained by setting $\mathcal{C}=\mathcal{R}$ :

$n$ d SPT orders and invertible topological orders with an anomaly-free algebraic higher symmetry $\mathcal{R}$ are classified by the invertible domain wall $\hat{\alpha}$ in $Z_{1}(\mathcal{R})$, such that its induced automorphisms $\alpha$ of $Z_{1}(\mathcal{R})$ satisfy $\alpha\left(A_{\widetilde{\mathcal{R}}}\right) \simeq A_{\tilde{\mathcal{R}}}$, where $A_{\widetilde{\mathcal{R}}}$ is the condensable algebra in $Z_{1}(\mathcal{R})$ that determines a boundary $\widetilde{\mathcal{R}}$ corresponding to the dual of the symmetry $\mathcal{R}$ (see Fig. 6).

This result generalizes the previous classification of SPT orders for higher symmetries [13-17].

\section{A HIGHER CATEGORY THEORY OF TOPOLOGICAL ORDERS IN HIGHER DIMENSIONS}

In this section, we present a review, a clarification, and an expansion of a higher category theory for topological orders in higher dimensions, based on Refs. [25-31]. Many notions of higher category and topological order will be introduced and explained for physics and mathematics audiences. Those notions will be used to understand algebraic higher symmetry and categorical symmetry, as well as to classify topological orders and SPT orders with those symmetries, in the rest of this paper. Readers who are familiar with higher category and topological order can skip this section.

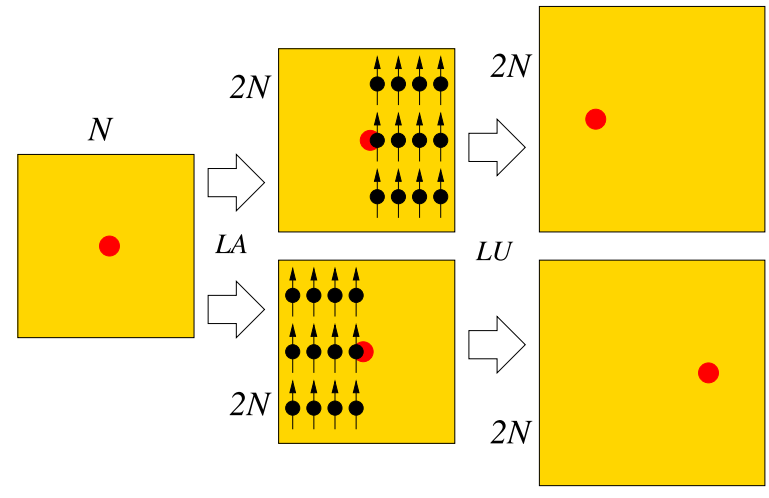

FIG. 9. There are two kind of equivalent relations: (1) finitedepth local unitary (LU) transformations and (2) local addition (LA) of product states.

\section{A. Topological orders as gapped liquid phases}

In this section, we give a microscopic description of topological order. Topological orders [36-38] are gapped liquid phases without symmetry. The notion of gapped liquid phases is introduced in Refs. [32,39,40]:

Definition $^{\text {ph }}$ 8. A gapped liquid phase is an equivalence class of gapped states, under the following two equivalence relations (see Fig. 9):

(1) two gapped states connected by a finite-depth local unitary transformation are equivalent;

(2) two gapped states differ by a stacking of product state are equivalent, where the degrees of freedom of the product state may have a nonuniform but bounded density.

If there is no symmetry, the local unitary transformation has no symmetry constraint, and the corresponding gapped liquid phases of local bosonic or fermionic systems are topological orders [36-38]. In the presence of finite internal symmetry, the local unitary transformation is required to commute with the symmetry transformations, and the corresponding gapped liquid phases include spontaneous symmetry breaking orders, symmetry protected trivial (SPT) orders [2,50,71], and symmetry enriched topological (SET) orders [32,72-80].

In this paper, we only consider bosonic systems with finite internal symmetries. We do not consider spacetime symmetries (such as time reversal and translation symmetries), nor continuous symmetry [such as $U(1)$ symmetry]. So in this paper, when we refer symmetry, we only mean finite internal symmetry.

We would like to remark that the above definition has an important feature. A gapped liquid phase with some noninvertible topological excitations on top of it is not a gapped liquid phase according to the definition. (The notion of noninvertible topological excitations is defined in the next section.) We note that the Hamiltonian here may not have translation symmetry. Thus it is hard to tell if the ground of a Hamiltonian has excitation in it or not. Using our above definition, a gapped liquid state is a ground state of a Hamiltonian that has no noninvertible topological excitations. However, a gapped liquid state may contain invertible topological excitations. In fact, two gapped liquid states differ by invertible topological 
excitations are very similar, and both can be viewed as proper ground states.

To see the above point, let us start with a gapped state of $N$ sites with a topological excitation in the middle. We may double the system size by stacking a product state of $N$ sites to the left half of the system, or to the right half of the system. Both operations are equivalence relations, and the resulting states of $2 \mathrm{~N}$ sites should be equivalent, i.e., be connected by a finite-depth local unitary transformation. However, in the presence of the noninvertible topological excitation, the excitation appears at left $1 / 4$ or right $1 / 4$ of the enlarged systems (see Fig. 9). Such two enlarged systems are not connected by finite-depth local unitary transformations, which can only move the noninvertible topological excitation by a finite distance. Thus, a gapped liquid state with some noninvertible topological excitations can no longer be viewed as a gapped liquid state.

However, a gapped liquid state with some invertible topological excitations can still be viewed as a gapped liquid state. This is because finite-depth local unitary transformations can move invertible topological excitations by a large distance across the whole system. Thus, the gapped liquid phases defined above may contain some invertible topological excitations.

References [25,26] outline a description of topological orders (i.e., without symmetry) in any dimensions, via braided fusion higher categories. Here, we would like to review and expand the discussions in Refs. $[25,26]$. We would like to remark that the needed higher category theory is still not fully developed, so our discussion here is just an outline. We hope that it can be a blueprint for further development. However, our discussions become rigorous at low dimensions (such as $1 \mathrm{~d}$ and $2 \mathrm{~d})$.

\section{B. Trivial, local, and topological excitations}

The reason that gapped liquid phases (which include topological orders) can be described by higher categories is that higher category is the natural language to describe excitations within a gapped liquid phase, as well as domain walls between different gapped liquid phases. To understand this connection, let us define excitation more carefully. We find that there two different ways to define types of excitations, which result in different kinds of higher category theories $[25,26]$. However, only the first definition of types and its associated higher category theory are more developed [29-31]. We will concentrate on this one.

We consider a gapped liquid state, which is the ground state of a local Hamiltonian $H$. As discussed in last section, a gapped liquid state does not contain any noninvertible topological excitations. To define excitations in $H$, for example, to define string-like excitations, we can add several trap Hamiltonians $\delta H_{\text {str }}\left(S_{a}^{1}\right)$, labeled by $a$, to $H$ such that $H+\sum_{a} \delta H_{\text {str }}\left(S_{a}^{1}\right)$ has an energy gap. $\delta H_{\text {str }}\left(S_{a}^{1}\right)$ is only nonzero along the string $S_{a}^{1}$. Here we require $\delta H_{\text {str }}\left(S_{a}^{1}\right)$ to be local along the string $S_{a}^{1}$. $\delta H_{\text {str }}\left(S_{a}^{1}\right)$ can be any operator, as long as its acts on the degrees of freedom near the string $S_{a}^{1}$. The ground state subspace $\mathcal{V}_{\text {fus }}\left(S_{1}^{1}, S_{2}^{1}, \ldots\right)$ of $H+\sum_{a} \delta H_{\text {str }}\left(S_{a}^{1}\right)$ (where is also called the fusion space) corresponds to stringlike excitations located at $S_{1}^{1}, S_{2}^{1}$, etc. (see Fig. 10).

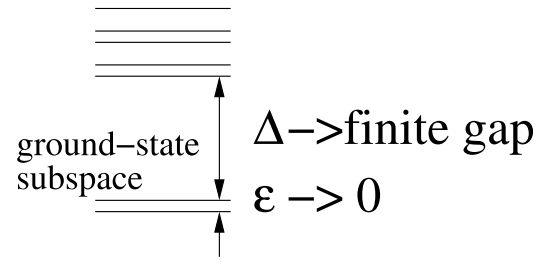

FIG. 10. The ground-state subspace below the energy gap $\Delta$. The energy splitting $\epsilon$ in the subspace approaches to 0 in thermodynamic limit, which the energy gap $\Delta$ remains nonzero.

If the ground-state subspace is stable (i.e., unchanged) against any small change of $\delta H_{\text {str }}\left(S_{a}^{1}\right)$, then we say the corresponding string on $S_{a}^{1}$ is a simple excitation (or a simple morphism in mathematics). If the ground-state subspace has accidental degeneracy (i.e., can be split by some small change of $\delta H_{\text {str }}\left(S_{a}^{1}\right)$; see Fig. 11), then we say the correspond string on $S_{a}^{1}$ is a composite excitation (or a composite morphism in mathematics). A composite excitation $I$ is a direct sum of several simple excitations

$$
I=i \oplus j \oplus \cdots .
$$

In other words, $I$ can be viewed as an accidental degeneracy of excitations $i, j, \ldots$ We see that different stringlike excitations can be labeled by different trap Hamiltonians $\delta H_{\text {str }}\left(S_{a}^{1}\right)$ (i.e., different nonlocal operators on $S_{a}^{1}$, s).

Definition $^{\text {ph }}$ 9. Excitations are something that can be trapped. In other words, excitations are described by the ground-state subspace of the Hamiltonian with traps.

The above definition give us too many different strings, and many of different strings actually have similar properties, so we would like to introduce a equivalence relation to simplify the types of strings. We define two strings labeled by $\delta H_{\text {str }}\left(S^{1}\right)$ and $\delta \tilde{H}_{\text {str }}\left(\tilde{S}^{1}\right)$ as equivalent, if we can deform $\delta H_{\text {str }}\left(S^{1}\right)$ into $\delta \tilde{H}_{\text {str }}\left(\tilde{S}^{1}\right)$ without closing the energy gap. The equivalence classes of the strings define the types of the strings. We would like to point out that if $S^{1}$ is an open segment, the corresponding string is equivalent to the trivial string $\mathbf{1}_{s}$ described by $\delta H_{\text {str }}\left(S^{1}\right)=0$, since we can shrink the string along $S^{1}$ to a point without closing the gap.

Definition $^{\text {ph }}$ 10. Two excitations are equivalent (i.e., are of the same type) if they can be connected by local-unitary transformations and by stacking of product states.

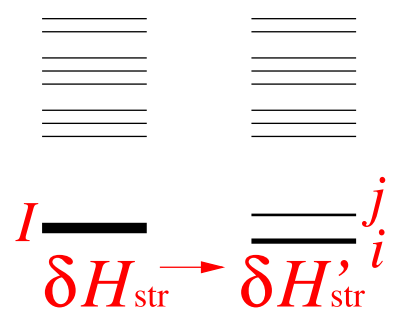

FIG. 11. The ground-state subspace of a composite excitation can be split by some change of trap Hamiltonian $\delta H_{\text {str }} \rightarrow \delta H_{\text {str. }}^{\prime}$. The ground-state subspace of a composite excitation $i$ can be viewed as a direct sum of the ground-state subspaces of excitations $j$ and $k$. In this sense, we can denote $I=i \oplus j$. 
We would like to remark that if the two excitations are defined on a closed submanifold, then we can define their equivalence by deforming their trap Hamiltonians into each other in the space of local trap Hamiltonians without closing the energy gap. The above definition is more general, since the local-unitary transformations and stacking of product states can be applied to a part of the submanifold that support the excitations, and we can examine if the two excitations turn into each other on the part of the submanifold.

Definition 11. An excitation is trivial if it is equivalent to the null excitation defined by a vanishing trap Hamiltonian.

Definition 12. An excitation is invertible if there exists another excitation such that the fusion of the two excitations is equivalent to a trivial excitation.

The above equivalence relation can also be phrased in a way similar to Definition 8:

Proposition 4. A type of excitations is an equivalence class of gapped ground states with added trap Hamiltonian acting on a $m$-dimensional subspace $S^{m}$, under the following two equivalence relations:

(1) two gapped states connected by a finite-depth local unitary transformation acting on the subspace $S^{m}$ are equivalent;

(2) two gapped states differ by a stacking of product states located on the subspace $S^{m}$ are equivalent.

We see that, when $m>0$, the excitations defined above are gapped liquid state on the subspace $S^{m}$, and there is no lower dimensional noninvertible excitations on $S^{m}$.

We also would like to introduce the notion of nonlocal equivalence and nonlocal type:

Definition $^{\text {ph }}$ 13. Two excitations are nonlocally equivalent (i.e., are of the same nl-type) if they can be connected by non-local-unitary transformations and by stacking of product states.

Definition $^{\text {ph }}$ 14. An excitation is local if it has the same nl-type as the null excitation.

We see that a trivial excitation is always a local excitation, but a local excitation may not be a trivial excitation.

Definition $^{\text {ph }}$ 15. An excitation is topological if it is nonlocal.

Again, the above nonlocal equivalence relation can also be phrased in a way similar to Definition 8:

Proposition 5. A nl-type of excitations is an equivalence class of gapped states with added trap Hamiltonian acting on a $m$-dimensional subspace $S^{m}$, under the following two equivalence relations:

(1) two gapped states connected by a nonlocal unitary transformation acting on the subspace $S^{m}$ are equivalent;

(2) two gapped states differ by a stacking of product states located on the subspace $S^{m}$ are equivalent.

We also believe the following:

Proposition 6. Two excitations have the same nl-type if and only if they can be connected by gapped or gapless domain walls. We note that the morphisms in higher category only correspond to gapped domain walls.

We would like to remark that for point-like excitations the notion of type and nl-type coincide.

Those different concepts of excitations were discussed in Ref. [25], where the nl-type was called elementary type and topological excitation was called elementary topological excitation. The local excitation was called descendant excitation in Ref. [25].

\section{Examples of excitations}

To illustrate the above concepts that we just introduced, let us consider a $2 \mathrm{~d} \mathbb{Z}_{2}$ topological order $[81,82]$ for bosons described by $2+1 \mathrm{D} \mathbb{Z}_{2}$ gauge theory.

Example 1. The $\mathbb{Z}_{2}$ topological order has four types of point-like excitations, labeled by $\mathbf{1}, e, m, f$, where $e$ is the $\mathbb{Z}_{2}$ charge, $m$ is the $\mathbb{Z}_{2}$ vortex, and $f$ is a fermion-the bound state of $e$ and $m . \mathbf{1}$ is a trivial point-like excitation. The $\mathbb{Z}_{2}$ topological order also has four nl-types of point-like excitations, labeled by $\mathbf{1}, e, m, f . \mathbf{1}$ is a local point-like excitation, and $e, m, f$ are topological point-like excitations.

The $\mathbb{Z}_{2}$ topological order has only one nl-type of stringlike excitations, which is a local string-like excitation. The $\mathbb{Z}_{2}$ topological order has six types of string-like excitations, generated by $\mathbf{1}_{s}, e_{s}, m_{s}, f_{s}$, with two additional types given by $f_{s} \otimes m_{s}=e_{s} \otimes f_{s}$ and $m_{s} \otimes f_{s}=f_{s} \otimes e_{s}:$

$$
\begin{aligned}
e_{s} \otimes e_{s} & =2 e_{s}, \\
m_{s} \otimes m_{s} & =2 m_{s}, \\
e_{s} \otimes m_{s} & =f_{s} \otimes m_{s}=e_{s} \otimes f_{s}, \\
m_{s} \otimes e_{s} & =m_{s} \otimes f_{s}=f_{s} \otimes e_{s} .
\end{aligned}
$$

The $e_{s}$-type of string-like excitation is formed by the $e$ particles, condensing into a $1 \mathrm{~d}$ phase of spontaneous $\mathbb{Z}_{2}$ symmetry-breaking state. We note that the $e$-particles have a mod- 2 conservation and an emergent $\mathbb{Z}_{2}$ symmetry. Similarly, the $m_{s}$-type of string-like excitation is formed by the $m$-particles, condensing into a $1 \mathrm{~d}$ phase of spontaneous $\mathbb{Z}_{2}$ symmetry-breaking state. The $f_{s}$-type of string-like excitation is formed by the $f$-particles, condensing into a $1 \mathrm{~d}$ topological superconducting phase (i.e., the $1 \mathrm{~d}$ invertible topological order of fermions where the string ends have Majorana zero modes [83]); $\mathbf{1}_{s}$ is the trivial string-like excitation. Although $\mathbf{1}_{s}, e_{s}, m_{s}, f_{s}$ are four different types of string-like excitations, they are all local string-like excitations, i.e., belong to the trivial nl-type of string-like excitations. We also comment that $f_{s}$ is an invertible string-like excitations, or the $e, m$ - exchange transparent domain wall; if we move $e$ throuch $f_{s}$, it becomes $m$ and vice versa.

Next we consider a $3 \mathrm{~d}$ trivial product state of bosons.

Example 2. Such a state has trivial nl-type of point-like, string-like, and membrane-like excitations; i.e., all excitations are local. It also has trivial type of point-like and stringlike, but it has nontrivial types of membrane-like excitations. In fact, those nontrivial types of membrane-like excitations correspond to $2 \mathrm{~d}$ topological orders. Thus, there are infinite types of membrane-like excitations corresponding to infinite different $2 \mathrm{~d}$ topological orders, even though the $3 \mathrm{~d}$ state has trivial topological order and is a trivial product state of bosons. All those membrane-like excitations are local but not trivial.

Remark 1. We remark that the our above description of $3 \mathrm{~d}$ trivial topological order is different from that in Refs. [29,30]. References [29,30] only include membrane excitations that correspond to $2 \mathrm{~d}$ topological orders with gappable boundary. There are still infinite type of membranes of this kind. In 
our description, the membrane excitations include both $2 \mathrm{~d}$ topological orders with gappable boundary and $2 \mathrm{~d}$ topological orders whose boundary cannot be gapped.

We see that to have a complete macroscopic description of trivial product state of bosons in $n$-dimensional space without symmetry, we need to classify $(n-1) \mathrm{d}$ topological orders of bosons, which correspond to types codimension-1 excitations. We also see that to have a complete macroscopic description of $n$ d topological order of bosons without symmetry, we need to classify $(n-1)$ d SET orders of bosons and fermions with symmetries (i.e., the emergent symmetry).

\section{Trivial topological order (the product states) and its excitations}

In the last section, we see that the types of dimension- $k$ excitations in a trivial product state in $n$-dimensional space correspond to topological orders (gapped liquid phases) in $k$ dimensional space. Thus, the study of the trivial topological order and its excitations of various dimensions allows us to understand topological orders in spatial dimensions less then $n$. This motivated us to develop a comprehensive theory of trivial topological order.

All trivial topological orders are product states and all product states belong to one phase, if there is no symmetry. We denote the trivial topological order in $n$-dimensional space as $\mathrm{I}^{n+1}\left(n+1\right.$ is the spacetime dimension). $\mathrm{I}^{n+1}$ is also referred as an object. Once we have the trivial topological order $\left.\right|^{n+1}$, we also have accidental degeneracy of several $I^{n+1}$ 's (i.e., several product states). We denote a gapped liquid phase formed by $m$ degenerate product states as $\underbrace{I^{n+1} \oplus \cdots \oplus \mathbf{I}^{n+1}}_{m \text { copies }}=\left.m\right|^{n+1}$. So, after the completion, the collection of trivial topological orders has objects $m l^{n+1}$. We refer $l^{n+1}$ as simple object and $m l^{n+1}(m>1)$ as composite object. We see that the composite object does not correspond to a stable phase, since the accidental $m$-fold degeneracy can be easily split by local perturbations in the Hamiltonian.

The collection of trivial topological orders in $(n+1) \mathrm{D}$ spacetime, $\left\{\left.m\right|^{n+1}\right\}$, is a set. However, the objects in the set have many relations. Two objects can be connected by a gapped codimension-1 domain wall $a:\left.\left.m_{1}\right|^{n+1} \rightarrow m_{2}\right|^{n+1}$, which is called an 1-morphism. For example, an 1-morphism $a:\left.\left.2\right|^{n+1} \rightarrow 3\right|^{n+1}$ can be represented as

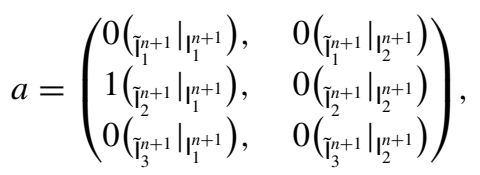

Physically, it means that there is a gapped domain wall between the first product state in $2 \mathrm{I}^{n+1}=\mathrm{I}_{1}^{n+1} \oplus \mathrm{I}_{2}^{n+1}$ and the second product state in $3 I^{n+1}=\tilde{I}_{1}^{n+1} \oplus \tilde{I}_{2}^{n+1} \oplus \tilde{I}_{3}^{n+1}$, and such a gapped domain wall is not degenerate. We denote such a gapped domain wall as $\left(\left.\tilde{\Gamma}_{2}^{n+1}\right|_{\left.\right|_{1} ^{n+1}}\right)$. All other domain walls between different product states have higher energy density or are gapless. In this paper, we do not consider gapless domain walls and we always assume gapless domain walls have infinite energy density.
We can have another 1-morphism $b:\left.\left.2\right|^{n+1} \rightarrow 3\right|^{n+1}$

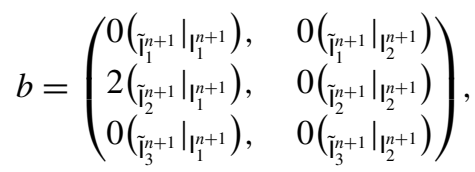

Physically, it means that there is a gapped domain wall between the first product state in $2 \mathrm{I}^{n+1}$ and the second product state in $\left.3\right|^{n+1}$, and such a gapped domain wall is twofold degenerate. So, we express the gapped domain wall as $\left(\left.\tilde{I}_{2}^{n+1}\right|_{1} ^{n+1}\right) \oplus\left(\left.\tilde{I}_{2}^{n+1}\right|_{1} ^{n+1}\right)=2\left(\left.\tilde{I}_{2}^{n+1}\right|_{1} ^{n+1}\right)$. The most general 1-morphism $c: 2 \mathrm{I}^{n+1} \rightarrow 3 \mathrm{I}^{n+1}$ has a form

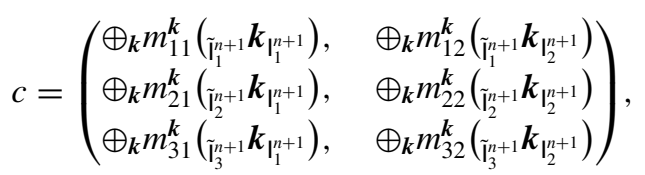

where $m_{i j}^{k} \in \mathbb{N}$. Here, for example, $\left(\tilde{I}_{2}^{n+1} \boldsymbol{k}_{l_{1}^{n+1}}\right)$ denotes a gapped domain wall between the first product state in $2 \mathrm{I}^{n+1}$ and the second product state in $3 \mathrm{I}^{n+1}$, and $\boldsymbol{k}$ labels different types of accidentally degenerate gapped domain wall between the two product states. $m_{i j}^{k}$ is the accidental degeneracy of the domain walls of the same type $\boldsymbol{k}$. We see that an 1-morphism is like a matrix that can also be added.

In particular, a 1-morphism $\boldsymbol{k}: \mathrm{I}^{n+1} \rightarrow \mathrm{I}^{n+1}$, denoted by $\left(\left.\right|^{n+1} \boldsymbol{k}_{\left.\right|^{n+1}}\right)$, is the codimension-1 excitation discussed in the last section, where $\boldsymbol{k}$ labels the different types of the excitations, as defined in Definition 10. Such an excitation corresponds to a topological order in $(n-1)$-dimensional space. We use $\operatorname{Hom}\left(I^{n+1}, I^{n+1}\right)$ to denote the collection of all morphisms from $\mathrm{I}^{n+1}$ to $\mathrm{I}^{n+1}$, which happen to be the collection of all topological orders in $(n-1)$-dimensional space. We remark that $\operatorname{Hom}\left(\mathrm{I}^{n+1}, \mathrm{I}^{n+1}\right)$ is also complete in the sense that it not only contains stable topological orders but also contains accidental degeneracy of topological orders. In other words, if $\boldsymbol{a}, \boldsymbol{b} \in \operatorname{Hom}\left(\mathrm{I}^{n+1}, \mathrm{I}^{n+1}\right)$, then the accidental degeneracy of $\boldsymbol{a}$ and $\boldsymbol{b}, \boldsymbol{a} \oplus \boldsymbol{b}$, is also in $\operatorname{Hom}\left(\mathrm{I}^{n+1}, \mathrm{I}^{n+1}\right)$. Thus, just like the collection of trivial topological orders $\left\{\left.m\right|^{n+1}\right\}, \operatorname{Hom}\left(\left.\right|^{n+1}, I^{n+1}\right)$ is also closed under the "degeneracy" operation $\oplus$.

We would like to point out that there is a 1-morphism in $\operatorname{Hom}\left(\mathrm{I}^{n+1}, \mathrm{I}^{n+1}\right)$ that corresponds to a codimension-1 trivial topological order (i.e., a product state in $(n-1)$-dimensional space or $n$-dimensional spacetime). We denote such a 1morphism as $\mathbf{I}^{n} \in \operatorname{Hom}\left(I^{n+1},\left.\right|^{n+1}\right)$.

Two codimension-1 topological orders $\boldsymbol{a}, \boldsymbol{b}$ may also be connected by a gapped domain wall of codimension-2: $\boldsymbol{k}$ : $\boldsymbol{a} \rightarrow \boldsymbol{b}$ (see Fig. 12). We call $\boldsymbol{k}$ a 2-morphism. To be precise, here, the "domain wall" really means types of domain walls. We regard two domain walls as equivalent if they differ only by local unitary transformations and local addition of product states on the wall. The collection of 2-morphisms from $\boldsymbol{a}$ to $\boldsymbol{b}$ is denoted as $\operatorname{Hom}(\boldsymbol{a}, \boldsymbol{b})$. We see that the collection of 2-morphisms from $\mathrm{I}^{n}$ to $\mathrm{I}^{n}$, $\operatorname{Hom}\left(\mathrm{I}^{n}, \mathrm{I}^{n}\right)$, is the collection of condimension-2 excitations, which are also topological orders in $(n-2)$-dimensional space. Such a collection also contains a product state (trivial topological order) in $(n-2)$ dimensional space, denoted as $\mathrm{I}^{n-1} \in \operatorname{Hom}\left(\mathrm{I}^{n}, \mathrm{I}^{n}\right)$. Also, the collection of 2-morphisms from $\boldsymbol{a}$ to $\boldsymbol{a}, \operatorname{Hom}(\boldsymbol{a}, \boldsymbol{a})$, is the 


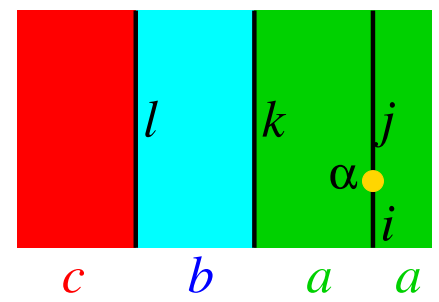

FIG. 12. The 2-dimensional excitations $a, b, c$ are objects. The 1-dimensional domain walls $\boldsymbol{i}, \boldsymbol{j}, \boldsymbol{k}, \boldsymbol{l}$ are 1 -morphisms. $\boldsymbol{\alpha}$ is a 2 morphism (domain wall) connecting two 1-morphisms $i$ and $j$. The fusion of domain walls $\boldsymbol{k}, \boldsymbol{l}$ between excitations $\boldsymbol{a}, \boldsymbol{b}, \boldsymbol{c}$ via the "glue" $\boldsymbol{b}$ is given by $\boldsymbol{l} \otimes \boldsymbol{k}$.

collection of codimension- 2 excitations on the codimension- 1 excitation $\boldsymbol{a}$.

We would like to remark that it is possible that $\operatorname{Hom}(\boldsymbol{a}, \boldsymbol{b})=0$, which means that there is no gapped domain wall between $\boldsymbol{a}$ and $\boldsymbol{b}$. Here 0 denotes the "zero" category, which can be roughly thought as the linearized and categorified version of the empty set.

The above discussion can be continued. This allows us to define 3-morphisms, 4-morphisms, etc. The $n$-morphisms correspond to codimension- $n$ or dimension-0 (i.e., pointlike) excitations. The point-like excitations are world lines in spacetime. The domain wall on world lines are $(n+1)$ mophisms. In general, a point-like excitation $\boldsymbol{p}$ (an $n$ morphism) may have degenerate ground states (of the Hamiltonian with traps). We denote the vector space of the degenerate ground states as $\mathcal{V}_{\text {fus }}(\boldsymbol{p}, \ldots)$, where ... represent other excitations which are fixed. Then a $(n+1)$-morphism $\boldsymbol{o}$ from one point-like excitations $\boldsymbol{p}_{1}$ to the other $\boldsymbol{p}_{2}$ (where the two excitations are near each other) is a linear operator acting near $\boldsymbol{p}_{1}$ and $\boldsymbol{p}_{2}$ from $\mathcal{V}_{\text {fus }}\left(\boldsymbol{p}_{1}, \ldots\right)$ and $\mathcal{V}_{\text {fus }}\left(\boldsymbol{p}_{2}, \ldots\right)$ : $\mathcal{V}_{\text {fus }}\left(\boldsymbol{p}_{1}, \ldots\right) \stackrel{\boldsymbol{o}}{\rightarrow} \mathcal{V}_{\text {fus }}\left(\boldsymbol{p}_{2}, \ldots\right)$. We denote such a $(n+1)-$ morphism as $\boldsymbol{o}: \boldsymbol{p}_{1} \rightarrow \boldsymbol{p}_{2}$.

Just like the objects (also called 0-morphisms), the morphisms also can be divided into two classes: the simple morphisms (which correspond to stable excitations whose ground state cannot be split by any local perturbations near the excitations) and composite morphisms (which correspond to unstable excitations with accidental degeneracy).

The objects (i.e., the 0 -morphisms), as well as $m$ morphisms can also fuse or compose. Let $\boldsymbol{a}, \boldsymbol{b}, \boldsymbol{c}$ be three $(m-1)$-morphisms, and $\boldsymbol{k} \in \operatorname{Hom}(\boldsymbol{a}, \boldsymbol{b})$ and $\boldsymbol{l} \in \operatorname{Hom}(\boldsymbol{b}, \boldsymbol{c})$ be two $m$-morphisms. Then, a composition of $\boldsymbol{k}$ and $\boldsymbol{l}$ is given by a $m$-morphism from $\boldsymbol{a}$ to $\boldsymbol{c}: \boldsymbol{l} \underset{\boldsymbol{b}}{\otimes} \boldsymbol{k} \in \operatorname{Hom}(\boldsymbol{a}, \boldsymbol{c})$. The subscript $\boldsymbol{b}$ indicates that $\boldsymbol{k}$ and $\boldsymbol{l}$ are fused together via the "glue" $\boldsymbol{b}$ (see Fig. 12). Figure 12 also has a dual representation, shown in Fig. 13.

Above, we discussed excitations of various dimensions in a trivial topological order. We may reverse the thinking and use all the excitations to characterize the trivial topological order, or more generally, a nontrivial topological order. This is equivalent to using higher categories to characterize topological orders or trivial orders. However, in order to use excitations to describe topological orders or trivial orders, the first issue one faces is weather to use type or use nl-type of excitations

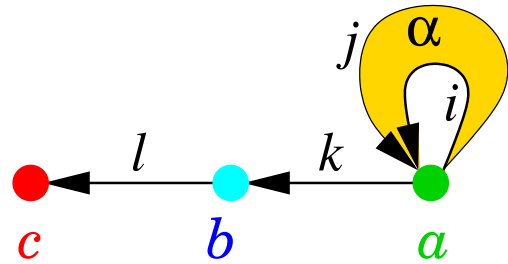

FIG. 13. A dual representation of Fig. 12. A higher category is a collection of vertices (objects), arrows (1-morphisms), oriented surfaces (2-morphisms), etc., connected in a certain way. In other words, a higher category is a simplicial complex

to construct higher categories. The notions of type and nltype were discussed in Refs. [25,26]. In physics, when we refer topological excitations, we usually mean the nl-types of excitations, which seems to suggest using nl-type to construct higher category. However, in mathematics, it is more natural to use types of excitations to build the higher categories that describe topological orders [29]. In some sense, nl-types are like the basis vectors in a vector space. The completion under "+" give rise to all the types which form the whole vector space. In higher category theory, such a completion corresponds to condensing the nl-types of excitations to construct all the types of excitations.

Definition $^{\text {ph }}$ 16. Descendent excitations are excitations of dimension 1,2,3, etc., which are obtained by condensing lower dimensional excitations.

The process of adding all the types of excitations in a category is called condensation completion, which is discussed in Ref. [29]. (Note that the condensation completion in Ref. [29] only includes defects that correspond to gapped liquid phases that have gappable boundaries; see Remark 1.) In this paper, we do a more general condensation completion that includes all the descendent excitations that correspond to all possible gapped liquid phases. In other words, we use types of all excitations to build the higher categories.

In $n$-dimensional space, the trivial topological order has dimension- $(n-1)$ excitations, dimension- $(n-2)$ excitations, etc. Those excitations can fuse (the $\otimes$ operation) and can have accidental degeneracy (the $\oplus$ operation). The excitations can also have domain walls between them (the morphisms). The collection of excitations plus those extra structures form a fusion $n$-category, which is denoted as $\operatorname{Hom}\left(\mathrm{I}^{n+1}, \mathrm{I}^{n+1}\right)$.

The precise definition of a fusion $n$-category is difficult to write down due to the lack of a universally accepted and well-developed model of weak $n$-categories. However, this is not the only problem. Recently, by ignoring this problem, Johnson-Freyd managed to solve other important problems and provided a workable definition in Ref. [30]. Because of its complexity, we choose to not to give Johnson-Freyd's definition but to provide a rough and physical intuition underlying the definition instead.

Definition $^{p h}$ 17. A fusion n-category is an n-category, which is $\mathbb{C}$-linear: the $n$-morphisms are required to form complex vector spaces; additive (with $\oplus$ operation); monoidal: with fusion $\otimes$ operation, which is compatible with the $\mathbb{C}$-linear and additive structures; semisimple (all composite object $\boldsymbol{x}$ has a unique decomposition $\boldsymbol{x}=\boldsymbol{a} \oplus \boldsymbol{b} \cdots)$ and the tensor unit is simple; condensation complete: the 
TABLE I. Correspondence between concepts in higher category and concepts in topological order $[25,26]$.

\begin{tabular}{|c|c|}
\hline Concepts in higher category & Concepts in physics \\
\hline Symmetric monoidal $(n+1)$-category $\mathrm{TO}_{\mathrm{af}}^{n+1}$ & $\begin{array}{l}\text { The collection of all } n \mathrm{~d} \text { anomaly-free topological orders, which can all be realized by } \\
\text { bosonic lattice model in the same dimension }\end{array}$ \\
\hline A simple object (0-morphism) of $\mathrm{TO}_{\mathrm{af}}^{n+1}$ & A topological order (with nondegenerate ground state on $S^{n}$ ) \\
\hline $\begin{array}{l}\text { Simple 1-morphisms of } \mathrm{TO}_{\mathrm{af}}^{n+1} \text { connecting } \\
\text { different objects }\end{array}$ & The types of domain wall between different topological orders \\
\hline $\begin{array}{l}\text { Simple 1-morphisms of } \mathrm{TO}_{\mathrm{af}}^{n+1} \text { connecting } \\
\text { the same object }\end{array}$ & $\begin{array}{l}\text { The types of codimension-1 topological excitations within a single topological order. They } \\
\text { can fuse (compose). }\end{array}$ \\
\hline Simple 2-morphisms of $\mathrm{TO}_{\mathrm{af}}^{n+1}$ & $\begin{array}{l}\text { The types of codimension- } 2 \text { topological excitations. They can fuse as well as braid (both } \\
\text { induced from composition). }\end{array}$ \\
\hline Simple $(n-1)$-morphisms of $\mathrm{TO}_{\mathrm{af}}^{n+1}$ & The types of string-like topological excitations \\
\hline Simple $n$-morphisms of $\mathrm{TO}_{\mathrm{af}}^{n+1}$ & The types of point-like topological excitations \\
\hline$(n+1)$-morphisms of $\mathrm{TO}_{\mathrm{af}}^{n+1}$ & The operators (instantons in spacetime) \\
\hline Composite morphisms & The types of topological excitations with accidental degeneracy \\
\hline Trivial morphisms & The types of excitations that can be created by local operators (the trivial excitations) \\
\hline
\end{tabular}

0-morphisms (the objects), 1-morphisms, 2-morphisms, etc. include all the decedent excitations; and satisfies certain fully dualizable condition amounts to the invariance of the physical reality by deforming and folding of the associated topological order.

Remark 2. Because our descendant excitations include topological orders whose boundary cannot be gapped, our definition of fusion $n$-category is different from that proposed by Refs. [29,30], where the descendent excitations only include topological orders with gappable boundary (see also Remark 1).

Since the excitations in a trivial topological order is surrounded by product states, we can add more product states to form a higher dimensional trivial topological order and view the same excitations as excitations in a higher dimension trivial topological order. In fact, we can view any excitations in a trivial topological order as excitations in an infinitedimensional trivial topological order. We can always braid the excitations in a trivial topological order by viewing the excitations as in an infinite dimensional trivial topological order. Since the excitations in an infinite dimensional trivial topological order have trivial braiding and exchange properties, those excitations form a symmetric fusion higher category with trivial exchange properties.

Proposition 7. The fusion $n$-category $\operatorname{Hom}\left(\mathrm{I}^{n+1}, \mathrm{I}^{n+1}\right)$, that describes the excitations in a trivial topological order $I^{n+1}$ in $n$-dimensional space, can always be promoted into a braided fusion $n$-category. In fact, such a braided fusion $n$-category is a symmetric fusion $n$-category with trivial exchange properties.

\section{E. The category of anomaly-free topological orders}

Definition $^{\text {ph }}$ 18. An anomaly-free topological order is a gapped liquid phase that can be realized by a local bosonic lattice models in the same dimension.

In a trivial topological order $\mathrm{I}^{n+2}(n+1)$-dimensional space, a type of codimension- 1 excitation correspond to an anomaly-free topological order in $n$-dimensional space. This is because we can remove the product state around a codimension- 1 excitation and view it as an anomaly-free topological order. Thus, $\operatorname{Hom}\left(I^{n+2},\left.\right|^{n+2}\right)$ is the set of anomaly-free $n$ d topological orders. Those $n d$ topological orders have excitations on them and have domain walls between them, which correspond to morphisms. They can also fuse $\otimes$ and have accidental degeneracies $\oplus$. Besides, we include all descendant excitations (condensation completion). Adding those structures to the set $\operatorname{Hom}\left(\left.\right|^{n+2},\left.\right|^{n+2}\right)$, we make it into a fusion $(n+1)$-category (see Table I), which leads to the definition of the first version of category of anomaly-free topological orders:

Definition 19. The category of anomaly-free topological orders in $n$-dimensional space is the fusion $(n+1)$-category given by $\operatorname{Hom}\left(\mathrm{I}^{n+2}, \mathrm{I}^{n+2}\right)$ where $\mathrm{I}^{n+2}$ is the trivial topological order (i.e., a product state) in $(n+1)$-dimensional space. We denote the category of anomaly-free topological orders as $\mathcal{T O}_{\text {af }}^{n+1}$.

In the above, we have defined anomaly-free topological orders via a microscopic approach, since we used the notion of product states and condensing low-dimensional excitations to construct descendant excitations. Can we define anomaly-free topological orders using only the macroscopic notions? Here we would like to point out that the anomaly-free topological orders have a defining macroscopic property called the principle of remote detectability $[25,84]$ :

Proposition 8. A topological order is anomaly-free if and only if any excitations of nontrivial nl-type can be detected remotely (such as via braiding) by some other excitations.

Here the nl-type also has a defining macroscopic property:

Proposition 9. Two excitations have the same nl-type if and only if they can be connected by gapped or gapless domain walls.

The gapped domain walls are the morphisms that we have discussed, while the gapless domain walls are not included in our discussion. Also the notion of "detecting remotely" is not defined carefully. This reveals the difficulty to define anomaly-free topological order macroscopically beyond 2-dimensional space. The above just points out a possible direction.

The category $\mathcal{T O}_{\mathrm{af}}^{n+1}$ include both topological phases (called stable topological orders) and correspond to simple 
objects in $\mathcal{T O}_{\mathrm{af}}^{n+1}$ ) and first-order phase transitions between two stable topological orders (called unstable topological orders). The stable topological order corresponds to simple object and the unstable topological order corresponds to composite object in $\mathcal{T O}_{\mathrm{af}}^{n+1}$. For example, the first-order phase transition point between two stable topological orders, the simple objects $A$ and $B$, correspond to the composite object $A \oplus B$, which can be viewed as the accidental degeneracy of the two stable topological orders $A$ and $B$. As a fusion category, the objects in $\mathcal{T O}_{\mathrm{af}}^{n+1}$ has this $\oplus$ operation.

The fusion higher category $\mathcal{T O}_{\mathrm{af}}^{n+1}$ has a special property, reflecting the following physics fact. The stacking of two stable anomaly-free topological orders $\mathrm{M}_{1}^{n+1}$ and $\mathrm{M}_{2}^{n+1}$ always gives us a third stable anomaly-free topological order $\mathrm{M}_{3}^{n+1}$, and the result does not depend on the order. Also note that the stacking is nothing but the fusion along $\mathrm{I}^{n+2}$. This means the following:

Proposition 10. The simple objects, $\mathrm{M}_{1}^{n+1}, \mathrm{M}_{2}^{n+1}, \mathrm{M}_{3}^{n+1}$, in $\mathcal{T O}_{\mathrm{af}}^{n+1}$ form a commutative monoid under the fusion $\underset{\mathrm{l}^{n+2}}{\otimes}$ :

$$
\mathrm{M}_{1}^{n+1} \underset{\mathrm{I}^{n+2}}{\otimes} \mathrm{M}_{2}^{n+1}=\mathrm{M}_{2}^{n+1} \underset{\left.\right|^{n+2}}{\otimes} \mathrm{M}_{1}^{n+1}=\mathrm{M}_{3}^{n+1} .
$$

$\underset{\left.\right|^{n+2}}{\otimes}$ is also abbreviated as $\otimes$. Since a commutative monoid may have a submonoid which is actually an Abelian group, anomaly-free topological orders have a subset of invertible topological orders $[25,41,42]$, which form an Abelian group under the stacking $\otimes$. All the invertible topological orders in $n$-dimensional space, plus their accidental degeneracies, also form a fusion $(n+1)$-category denoted as $\mathcal{T O}_{\mathrm{inv}}^{n+1}$.

From the above discussion, we see that we can ignore all the unstable topological orders and restrict ourselves to only stable topological orders (which is more natural from a physics point of view). After dropping all the composite objects from $\mathcal{T O}_{\mathrm{af}}^{n+1}$, we obtain a monoidal $(n+1)$-category $\mathrm{TO}_{\mathrm{af}}^{n+1}$. The objects in $\mathrm{TO}_{\mathrm{af}}^{n+1}$ still support the stacking $\otimes$ operation, but do not support the accidental degeneracy (or first-order phase transition) $\oplus$ operation. Thus, $\mathrm{TO}_{\mathrm{af}}^{n+1}$ is monoidal but not fusion.

\section{F. The category of anomalous topological orders}

In the last section, we see that $\operatorname{Hom}\left(I^{n+2}, I^{n+2}\right)$ gives rise to the collection of anomaly-free (stable and unstable) topological orders in $n$-dimensional space. Similarly, $\operatorname{Hom}\left(\mathrm{I}^{n+2}, \mathrm{M}^{n+2}\right)$ gives rise to the collection of gapped boundaries of a $(n+1) \mathrm{d}$ topological order described by $\mathrm{M}^{n+2}$. Those gapped domain walls have domain walls (morphisms) between them, as well as accidental degeneracy $\oplus$ operation, but they do not have stacking $\otimes$ operation. However, we can fuse the morphisms in $\operatorname{Hom}\left(\mathrm{I}^{n+2}, \mathrm{I}^{n+2}\right)$ to $\operatorname{Hom}\left(\mathrm{I}^{n+2}, \mathrm{M}^{n+2}\right)$ from right, and fuse the morphisms in $\operatorname{Hom}\left(\mathrm{M}^{n+2}, \mathrm{M}^{n+2}\right)$ to $\operatorname{Hom}\left(\mathrm{I}^{n+2}, \mathrm{M}^{n+2}\right)$ from left. Both $\operatorname{Hom}\left(\mathrm{I}^{n+2}, \mathrm{I}^{n+2}\right)$ and $\operatorname{Hom}\left(\mathrm{M}^{n+2}, \mathrm{M}^{n+2}\right)$ are fusion $(n+1)-$ categories. Thus, Hom $\left(\mathrm{I}^{n+2}, \mathrm{M}^{n+2}\right)$ is a right module of fusion $(n+1)$-category Hom $\left(\mathrm{I}^{n+2}, \mathrm{I}^{n+2}\right)$ and a left module of fusion $(n+1)$-category $\operatorname{Hom}\left(\mathrm{M}^{n+2}, \mathrm{M}^{n+2}\right)$.

As a collection of gapped boundaries of a $(n+1) \mathrm{d}$ anomaly-free topological order $\mathrm{M}^{n+2}, \operatorname{Hom}\left(\mathrm{I}^{n+2}, \mathrm{M}^{n+2}\right)$ supports the $\oplus$ operation but does not support the $\otimes$ operation.
In order to allow the staking $\otimes$ operation, we consider a collection of $\operatorname{Hom}\left(\mathrm{I}^{n+2}, \mathrm{M}^{n+2}\right)$ for all different $\mathrm{M}^{n+2}$; i.e., we consider all the gapped boundaries of all $(n+1) \mathrm{d}$ anomalyfree topological orders. Such an enlarged collection support the stacking $\otimes$ operation, by stacking both boundary and bulk. However, in the enlarged collection $\otimes$ operation becomes messy, since there are two kinds of accidental degeneracies: the accidental degeneracies of the boundary and the accidental degeneracies of the bulk, suggesting that there are two kinds of $\oplus$ operations. To keep things simple, we will drop all the unstable gapped boundaries and all the unstable bulk topological order; i.e., we restrict $\mathrm{M}^{n+2}$ to be simple objects and consider only simple 1-morphisms in $\operatorname{Hom}\left(\mathrm{I}^{n+2}, \mathrm{M}^{n+2}\right)$. In this way, we obtain the following:

Definition 20. The category of potential anomalous topological orders in $n$-dimensional space is the monoidal $(n+1)$-category given by the union of all $\operatorname{Hom}\left(I^{n+2}, \mathrm{M}^{n+2}\right)$ 's after dropping all the composition 1-morphisms, where $\mathrm{I}^{n+2}$ is the unit object in $\mathrm{TO}_{\mathrm{af}}^{n+2}$ and $\mathrm{M}^{n+2}$ is a simple object in $\mathrm{TO}_{\mathrm{af}}^{n+2}$. It is a right module over fusion $(n+1)$-category $\operatorname{Hom}\left(\mathrm{I}^{n+2}, \mathrm{I}^{n+2}\right)$, a left module over fusion $(n+1)$-category $\mathcal{M}^{n+1}=\operatorname{Hom}\left(\mathrm{M}^{n+2}, \mathrm{M}^{n+2}\right)$, and thus a bimodule. Here $\mathrm{M}^{n+2}$ is a stable anomaly-free topological order in $(n+1)$ dimensional space, which determines the anomaly. We denote the category of anomalous topological orders as $\mathrm{TO}^{n+1}$. Such a category describes all the gapped boundaries of all the anomaly-free topological orders

\section{G. Invertible domain wall between topological orders}

We have seen that the collection of domain walls (plus the excitations on the walls and their $\otimes, \oplus$ operations) in a stable $n$ d topological orders $\mathrm{C}$ is given by $\operatorname{Hom}(\mathrm{C}, \mathrm{C})$. In fact, $\mathcal{C}=\operatorname{Hom}(\mathrm{C}, \mathrm{C})$ is a fusion $n$-category. The objects in $\mathcal{C}$ (the domain walls) support the fusion $\otimes$ operation. Roughly, an object $a$ (a domain wall) is invertible if there exist another object $b$ such that $a \otimes b \simeq b \otimes a \simeq \mathrm{I}$, where $\mathrm{I}$ is the trivial object (the unit of $\otimes$ operation).

Let us give a more careful definition. An invertible $0 \mathrm{~d}$ domain wall $\hat{\gamma}$ between two $1 \mathrm{~d}$ topological orders $\mathrm{A}^{1}$ and $\mathrm{B}^{1}$ is well defined (see Section 4.3 in Ref. [26]) and is denoted by $\mathrm{A}^{1} \stackrel{\hat{\gamma}}{\simeq} \mathrm{B}^{1}$ or $\hat{\gamma}: \mathrm{A}^{1} \simeq \mathrm{B}^{1}$. Higher dimensional invertible domain walls are defined by induction:

Definition 21. A gapped domain wall $\mathrm{M}^{n-1}$ between two gapped walls $\mathrm{A}^{n}$ and $\mathrm{B}^{n}$ is called invertible if there is a gapped domain wall $\mathrm{N}^{n-1}$ between $\mathrm{B}^{n}$ and $\mathrm{A}^{n}$ such that there exist an invertible gapped domain wall between $\mathrm{M}^{n-1} \otimes \mathrm{N}^{n-1}$ and $\operatorname{id}_{\mathrm{A}^{n}}$, i.e., $\mathrm{M}^{n-1} \otimes \mathrm{N}^{n-1} \simeq \mathrm{id}_{\mathrm{A}^{n}}$, and one between $\mathrm{N}^{n-1} \otimes$ $\mathrm{M}^{n-1}$ and id $\mathrm{B}^{n}$, i.e., $\mathrm{N}^{n-1} \otimes \mathrm{M}^{n-1} \simeq \mathrm{id}_{\mathrm{B}^{n}}$.

The invertible domain wall will be used extensively later.

\section{H. Looping and delooping}

Looping and delooping operations reveal the layered structures in higher categories. From an $n$-category we can construct a fusion $(n-1)$-category via a process called looping (see Fig. 13 where the morphisms are viewed as paths and loops): 
Definition 22. Given an $n$-category $\mathcal{C}$, we choose an object $\boldsymbol{a}$ (the "base point"). We can construct a fusion $(n-1)$ category, denoted as $\Omega_{a} \mathcal{C}$, whose objects are given by the morphisms $\boldsymbol{k}: \boldsymbol{a} \rightarrow \boldsymbol{a}$. In other words $\Omega_{\boldsymbol{a}} \mathcal{C}=\operatorname{Hom}(\boldsymbol{a}, \boldsymbol{a})$. If $\mathcal{C}$ is a fusion $n$-category, we usually choose the base point to be the unit of fusion $\mathbf{1}_{\mathcal{C}}: \Omega \mathcal{C}=\operatorname{Hom}\left(\mathbf{1}_{\mathcal{C}}, \mathbf{1}_{\mathcal{C}}\right)$, and $\Omega \mathcal{C}$ becomes a braided fusion $n$-category.

To apply the looping to a physical situation, let us consider a single simple object $\mathrm{C}^{n+1}$ in $\mathrm{TO}^{n+1}$, which corresponds to an $n \mathrm{~d}$ gapped boundary of an anomaly-free topological order $\mathrm{M}^{n+2}=\operatorname{Bulk}\left(\mathrm{C}^{n+1}\right)$ in $(n+1)$-dimensional space [see (41)]. $\mathrm{C}^{n+1}$ is also an $n \mathrm{~d}$ anomalous topological order. For special case $\mathrm{M}^{n+2}=\mathrm{I}^{n+2}, \mathrm{C}^{n+1}$ becomes an $n \mathrm{~d}$ anomaly-free topological order. $\operatorname{Hom}\left(\mathrm{C}^{n+1}, \mathrm{C}^{n+1}\right)$ is the collection of $(n-1) \mathrm{d}$ excitations on the boundary. Here we include the morphisms, as well as the $\otimes$ and $\oplus$ operations to view $\operatorname{Hom}\left(\mathrm{C}^{n+1}, \mathrm{C}^{n+1}\right)$ as a fusion $n$-category, denoted by $\mathcal{C}^{n}=\operatorname{Hom}\left(\mathrm{C}^{n+1}, \mathrm{C}^{n+1}\right)$. Thus, $\mathcal{C}^{n}$ describe all the codimension-1, codimension-2, etc., excitations on the $n$ d boundary $\mathrm{C}^{n+1}$

The unit object under $\otimes$ in $\mathcal{C}^{n}$ is denoted as $\mathbf{1}_{\mathcal{C}^{n}}=$ $\mathrm{id}_{\mathrm{C}^{n+1}}$, which is the trivial codimension-1 excitations in $\mathrm{C}^{n+1}$. Then the looping $\Omega \mathcal{C}^{n}=\operatorname{Hom}\left(\mathbf{1}_{\mathcal{C}^{n}}, \mathbf{1}_{\mathcal{C}^{n}}\right)$ is a fusion $(n-1)$ category, which describes the codimension- 2 excitations on the $n \mathrm{~d}$ boundary $\mathrm{C}^{n+1}$. Those excitations can also braid and $\Omega \mathcal{C}^{n}$ is in fact a braided fusion $(n-1)$-category.

We see that the looping of a fusion category $\mathcal{C}^{n}$ is obtained by dropping the objects (the codimension- 1 excitations) and include only the morphisms of the trivial object (the codimension-2 excitations). The looping operation can be continued, and the commutativity increases. For example, $\Omega \Omega \mathcal{C}^{n} \equiv \Omega^{2} \mathcal{C}^{n}$ is a symmetric fusion $(n-2)$-category.

There is reverse process of looping, called delooping (see Fig. 13). From a fusion $n$-category, we can construct an $(n+1)$-category via delooping:

Definition 23. Given an fusion $n$-category $\mathcal{C}$, we can construct a $(n+1)$-category, denoted as $\Sigma_{*} \mathcal{C}$, which has only one object $*$ and whose morphisms are given by the objects of $\mathcal{C}$. In other words, $\operatorname{Hom}(*, *)=\mathcal{C}$. We can complete $\Sigma_{*} \mathcal{C}$ by adding the composite objects $* \oplus * \cdots$, to obtain an additive $(n+1)$-category with $\oplus$ operation. We can also do a condensation completion, by adding objects (the gapped liquid phases) formed by the codimenson-1 excitations (i.e., the 1morphisms of $\Sigma_{*} \mathcal{C}$ ), the codimenson-2 excitations (i.e., the 2-morphisms of $\left.\Sigma_{*} \mathcal{C}\right)$, etc. The resulting $(n+1)$-category is called the delooping of $\mathcal{C}$ and is denoted by $\Sigma \mathcal{C}$.

Remark 3. Our definition of delooping is compatible with Definition $^{\text {ph }} 17$ (see also Remarks 1 and 2) and is different from that in Refs. [29,30].

As an application to physics, let us consider a braided fusion $(n-1)$-category $\mathrm{C}^{n-1}$ that describes the codimension2 and higher excitations in $n$-dimensional space. Then the delooping $\Sigma_{\left.\right|^{n}} \mathrm{e}^{n-1}$ is the fusion $n$-category with only one object $I^{n}$, which corresponds to the trivial codimension-1 excitation in the $n$-dimensional space. We can do a condensation completion by adding $\mathrm{I}^{n} \oplus \mathrm{I}^{n} \ldots$, as well as all the descendant codimension-1 excitations, obtained from condensing codimension-2 and higher excitations. The resulting fusion $n$-category is $\Sigma \mathrm{C}^{n-1}$. If we can add a braiding structure to $\Sigma \mathrm{C}^{n-1}$, making it a braided fusion $n$-category, then the delooping plus condensation completion can be continued $\Sigma \Sigma \mathcal{C}^{n-1}=\Sigma^{2} \mathrm{C}^{n-1}$ is a fusion $(n+1)$-category.

We note that excitations in a trivial topological order $\left.\right|^{n+1}$ in $n$-dimensional space are described by a fusion $n$-category $\operatorname{Hom}\left(\mathrm{I}^{n+1}, \mathrm{I}^{n+1}\right)$. It contains $(n-1) \mathrm{d},(n-2) \mathrm{d}, \ldots, 0 \mathrm{~d}$ excitations. If we drop the $(n-1) \mathrm{d}$ excitations, the remaining $(n-2) \mathrm{d}, \ldots, 0 \mathrm{~d}$ excitations correspond to excitations in trivial topological order $\mathrm{I}^{n}$ in $(n-1)$-dimensional space and are described by $\operatorname{Hom}\left(I^{n}, I^{n}\right)$. This way we find

$$
\Omega \operatorname{Hom}\left(I^{n+1}, I^{n+1}\right)=\operatorname{Hom}\left(I^{n}, I^{n}\right) \text {. }
$$

All the excitations in trivial topological order are descendent excitations. Thus, if we add one layer of descendent excitations in one higher dimension, we obtain excitations of a trivial topological order in one higher dimension. Therefore, we have

$$
\Sigma \operatorname{Hom}\left(\mathrm{I}^{n}, \mathrm{I}^{n}\right)=\operatorname{Hom}\left(\mathrm{I}^{n+1}, \mathrm{I}^{n+1}\right) \text {. }
$$

We note that the codimension- 1 excitations in a trivial topological order is embedded in a product state in 1 higher dimension. We can also view the same excitation as embedded in a product state in 2 higher dimension. In this case, the excitation becomes codimension- 2 and can braid. Thus, $\operatorname{Hom}\left(\mathrm{I}^{n+1}, \mathrm{I}^{n+1}\right)$ can also be viewed as a braided fusion $n$ category, and we can perform delooping. In fact, the braiding is trivial, and $\operatorname{Hom}\left(\mathrm{I}^{n+1}, \mathrm{I}^{n+1}\right)$ can be viewed as a symmetric fusion $n$-category.

Since $\operatorname{Hom}\left(\mathrm{I}^{n+2}, \mathrm{I}^{n+2}\right)=\mathcal{T O}_{\mathrm{af}}^{n+1}$ is the fusion higher category of anomaly-free topological orders in $n$-dimensional space, we find that

$$
\Omega \mathcal{T O} \mathrm{O}_{\mathrm{af}}^{n+1}=\mathcal{T O}_{\mathrm{af}}^{n}, \quad \Sigma \mathcal{T O}_{\mathrm{af}}^{n}=\mathcal{T O}_{\mathrm{af}}^{n+1} .
$$

We note that in 0-dimensional space, the category of anomaly-free topological order has only one simple object $\mathbf{I}^{1}$, which corresponds to a single quantum state $|\psi\rangle$. The set of 1 -morphisms $\operatorname{Hom}\left(\mathrm{I}^{1}, \mathrm{I}^{1}\right)=\mathbb{C}$ is the set of 1 -by- 1 complex matrices. We see that the category of anomaly-free topological orders in 1-dimensional space is given by $\mathcal{V e c}$ - the category of complex vector spaces:

$$
\mathcal{T O}_{\mathrm{af}}^{1}=\Sigma \mathbb{C}=\mathcal{V e c} .
$$

The higher category of $n$-vector spaces is given by the iterated delooping

$$
n \mathcal{V e c}=\Sigma^{n-1} \mathcal{V e c} .
$$

Remark 4. Our definition of $n \mathcal{V e c}$ is different from that in Ref. [29] for $n>2$ (recall Remark 3). We suspect that the difficulty of defining $n \mathcal{V}$ ec mathematically might be due to the complexity of higher topological orders.

We see that the category of anomaly-free topological orders is given by

$$
\mathcal{T O}_{\mathrm{af}}^{n+1}=\Sigma^{n} \mathcal{V e c}=(n+1) \mathcal{V e c} .
$$

We would like to remark that the fusion $(n+1)$-category $\mathcal{T O}_{\mathrm{af}}^{n+1}=\operatorname{Hom}\left(\mathrm{I}^{n+2}, \mathrm{I}^{n+2}\right)=(n+1) \mathcal{V e c}$ can also be promoted into a braided fusion $(n+1)$-category, which is actually, a symmetric fusion $(n+1)$-category with trivial exchange property (see Proposition 7). After we promote $(n+1) \mathcal{V e c}$ to a braided fusion $(n+1)$-category, we can denote it as $(n+1)$ Vec. In other words, $(n+1) \mathcal{V e c}$ is the 
braided fusion $(n+1)$-category obtained from the fusion $(n+1)$-category $(n+1) \mathcal{V e c}$ by adding the trivial braiding structure (which is always doable).

Consider an anomaly-free topological order $\mathrm{M}^{n+1} \in$ $\mathcal{T O}_{\mathrm{af}}^{n+1}$. Its excitations are described by a fusion $n$ category $\mathcal{M}^{n}=\operatorname{Hom}\left(\mathrm{M}^{n+1}, \mathrm{M}^{n+1}\right)$. The objects in $\mathcal{M}^{n}$ are codimension-1 excitations, which cannot braid and cannot be remotely detected by any excitations. Thus, according to Proposition 8 , those codimension-1 excitations must all have the trivial nl-type. We believe the following:

Proposition 11. All the excitations with the trivial nl-type are descendant excitations, coming from the condensations of lower dimensional excitations.

Thus, the codimension- 1 excitations in an anomaly-free topological order are all descendant excitations. Dropping those codimension-1 excitations gives us the looping $\Omega \mathcal{M}^{n}$. The delooping of $\Omega \mathcal{M}^{n}$ adds back those descendant codimension-1 excitations. We find the following:

Proposition 12. The excitations in an anomaly-free topological order described by fusion $n$-category $\mathcal{M}^{n}$ satisfy the following relation:

$$
\mathcal{M}^{n}=\Sigma \Omega \mathcal{M}^{n}
$$

The reverse may not be true: A fusion $n$-category $\mathcal{M}^{n}$ satisfying $\mathcal{M}^{n}=\Sigma \Omega \mathcal{M}^{n}$ may not describe the excitations in an anomaly-free topological order.

We see that the category of anomaly-free topological orders $\mathcal{T O}_{\mathrm{af}}^{n+1}=(n+1) \mathcal{V e c}$ is a fusion $(n+1)$-category. Since the condensation completion always include excitations induced by condensing the trivial excitations and $n \mathcal{V e c}$ is formed only by those excitations induced by condensing the trivial excitations, we find the following:

Corollary 1. A fusion $n$-category $\mathcal{C}^{n}$ is a bimodule of $n \mathcal{V e c}$

$$
\mathcal{C}^{n} \otimes n \mathcal{V e c}=n \mathcal{V e c} \otimes \mathcal{C}^{n}
$$

We also have Corollary 2.

Corollary 2. The $n$-category $\mathcal{M}^{n}$ that describes the excitations in an anomaly-free topological order $\mathrm{M}^{n+1} \in \mathcal{T O}_{\mathrm{af}}^{n+1}$, $\mathcal{M}^{n}=\operatorname{Hom}\left(\mathrm{M}^{n+1}, \mathrm{M}^{n+1}\right)$, is a fusion $n$-category.

This is because the $n$-category $\mathcal{M}^{n}$ contains all the descendent excitations.

\section{Boundary-bulk relation}

Consider an anomaly-free stable topological order $\mathrm{M}^{n+2}$ in $(n+1)$-dimensional space, $\mathrm{M}^{n+2} \in \mathrm{TO}_{\mathrm{af}}^{n+2}$, and its gapped boundaries. The $(n+1) \mathrm{d}$ bulk topological order and the $n \mathrm{~d}$ gapped boundaries have a very direct relation. References [25-27] proposed a holographic principle for this boundary-bulk relation: Boundary uniquely determines bulk. The boundary-bulk relation has several versions, which differ in mathematical details.

In the first version, we consider a linear $(n+1)$-category $\mathcal{B}^{n+1}$ and a fusion $(n+1)$-category $\mathcal{M}^{n+1}$ that acts on $\mathcal{B}^{n+1}$ from left. $\mathcal{B}^{n+1}$ is also a right module over the fusion $(n+1)$ category $\operatorname{Hom}\left(I^{n+2}, I^{n+2}\right)$. The pair $\left(\mathcal{B}^{n+1}, \mathcal{M}^{n+1}\right)$ describes a category of all gapped boundaries of an $(n+1) \mathrm{d}$ anomaly-free topological order in $\mathrm{TO}_{\mathrm{af}}^{n+2}$. We believe the following:
Proposition 13. There is only one anomaly-free topological order $\mathrm{M}^{n+2}$ in $\mathrm{TO}_{\mathrm{af}}^{n+2}$, which gives rise to the category of the gapped boundaries

$$
\left(\mathcal{B}^{n+1}, \mathcal{M}^{n+2}\right)=\left(\operatorname{Hom}\left(\mathrm{I}^{n+2}, \mathrm{M}^{n+2}\right), \operatorname{Hom}\left(\mathrm{M}^{n+2}, \mathrm{M}^{n+2}\right)\right) .
$$

We would like to point that the pair $\left(\mathcal{B}^{n+1}, \mathcal{M}^{n+2}\right)$ not only uniquely determines the bulk topological order $\mathrm{M}^{n+2}$ but also gives extra information about how the bulk is connected to the boundary. If we ignore such information, we believe that the linear $(n+1)$-category of the gapped boundaries can already uniquely determines the bulk topological order:

Proposition 14. There is only one anomaly-free topological order $\mathrm{M}^{n+2}$ in $\mathrm{TO}_{\mathrm{af}}^{n+2}$, which gives rise to the linear $(n+1)$-category for the gapped boundaries

$$
\mathcal{B}^{n+1}=\operatorname{Hom}\left(\mathrm{I}^{n+2}, \mathrm{M}^{n+2}\right) .
$$

In the second version, we consider a particular gapped boundary $\mathrm{C}^{n+1} \in \mathcal{B}^{n+1}$. Now $\operatorname{Hom}\left(\mathrm{M}^{n+2}, \mathrm{M}^{n+2}\right)$ does not act within $\mathrm{C}^{n+1}$ since the fusion with excitations in $\operatorname{Hom}\left(\mathrm{M}^{n+2}, \mathrm{M}^{n+2}\right)$ (i.e., the $n \mathrm{~d}$ excitations in $\mathrm{M}^{n+2}$ ) may change $\mathrm{C}^{n+1}$ to some other boundaries $\tilde{\mathrm{C}}^{n+1}$. However, the $(n-1) \mathrm{d}$ excitations in $\mathrm{M}^{n+2}$ act within $\mathrm{C}^{n+1}$. The $n$-category of all the $(n-1) \mathrm{d}$ excitations is given by Hom $\left(\mathrm{id}_{\mathrm{M}^{n+2}}, \mathrm{id}_{\mathrm{M}^{n+2}}\right)$, where $\operatorname{id}_{M^{n+2}} \in \operatorname{Hom}\left(\mathrm{M}^{n+2}, \mathrm{M}^{n+2}\right)$ is the unit morphism (that corresponds to the trivial $n \mathrm{~d}$ excitation in the bulk topological order $\left.\mathrm{M}^{n+2}\right)$. In fact, $\operatorname{Hom}\left(\mathrm{id}_{\mathrm{M}^{n+2}}, \mathrm{id}_{\mathrm{M}^{n+2}}\right)$ is a braided fusion category, which is actually defined as the looping $\Omega \operatorname{Hom}\left(\mathrm{M}^{n+2}, \mathrm{M}^{n+2}\right)$. Therefore, there is a braided fusion $n$ category $\mathcal{M}^{n}=\Omega \operatorname{Hom}\left(\mathrm{M}^{n+2}, \mathrm{M}^{n+2}\right)$ that acts on $\mathrm{C}^{n+1}$. A gapped boundary is described by a pair $\left(\mathrm{C}^{n+1}, \mathcal{M}^{n}\right)$. We believe that the pair $\left(\mathrm{C}^{n+1}, \mathcal{M}^{n}\right)$ uniquely determines the bulk topological order and how the bulk topological order is connected to the boundary. If we ignore the information about how the bulk is connected to the boundary, we believe that $\mathrm{C}^{n+1} \in \operatorname{Hom}\left(\mathrm{I}^{n+2}, \mathrm{M}^{n+2}\right)$ uniquely determines the bulk topological order:

Proposition 15. There is only one anomaly-free topological order $\mathrm{M}^{n+2}$ in $\mathrm{TO}_{\mathrm{af}}^{n+2}$, which gives rise to the boundary

$$
\mathrm{C}^{n+1} \in \operatorname{Hom}\left(\mathrm{I}^{n+2}, \mathrm{M}^{n+2}\right) \text {. }
$$

We denote such boundary-bulk relation as

$$
\operatorname{Bulk}\left(\mathrm{C}^{n+1}\right)=\mathrm{M}^{n+2} \text {. }
$$

The above is the accurate meaning of boundary uniquely determines bulk.

$\mathcal{C}^{n}$ can determine the boundary topological order $\mathrm{C}^{n+1}$ up to an invertible topological order. Since we believe that all invertible topological orders are anomaly-free, the excitations $\mathcal{C}^{n}=\operatorname{Hom}\left(\mathrm{C}^{n+1}, \mathrm{C}^{n+1}\right)$ in the boundary topological order $\mathrm{C}^{n+1}$ can already determine the bulk topological order $\mathrm{M}^{n+2}$. We obtain the following:

Proposition 16. For any fusion $n$-category $\mathcal{C}^{n}$, there is only one anomaly-free topological order $\mathrm{M}^{n+2}$ in $\mathrm{TO}_{\mathrm{af}}^{n+2}$ admitting a boundary $\mathrm{C}^{n+1} \in \operatorname{Hom}\left(\mathrm{I}^{n+2}, \mathrm{M}^{n+2}\right)$ such that

$$
\mathcal{C}^{n}=\Omega \mathrm{C}^{n+1} \text {. }
$$

We denote such boundary-bulk relation as

$$
\operatorname{bulk}\left(\mathcal{C}^{n}\right)=\mathrm{M}^{n+2}
$$

Here, we have assumed the following. 
Proposition 17. A fusion $n$-category $\mathcal{C}^{n}$ can always be realized by the excitations in a potentially anomalous topological order $\mathrm{C}^{n+1}$ such that $\mathcal{C}^{n}=\Omega \mathrm{C}^{n+1}$.

The above result was shown for $n=1$ case. Given a fusion category $\mathcal{C}$, we can explicitly construct a $2 \mathrm{~d}$ string-net liquid state [34] that has a boundary realizing the fusion category $\mathcal{C}$ [35]. For $n>1$, the general construction is sketched in Proposition 21.

In the third version, we only consider the excitations on a particular gapped boundary $\mathrm{C}^{n+1} \in \mathcal{B}^{n+1}$, and instead of determining bulk topological orders, we ask only whether boundary excitations can determine bulk excitations. The boundary excitations are described by a fusion $n$-category $\mathcal{C}^{n}=\operatorname{Hom}\left(\mathrm{C}^{n+1}, \mathrm{C}^{n+1}\right)$. Again Hom $\left(\mathrm{M}^{n+2}, \mathrm{M}^{n+2}\right)(n \mathrm{~d}$ excitations in $\mathrm{M}^{n+2}$ ) does not act within $\mathcal{C}^{n}$. However, the $(n-1) \mathrm{d}$ excitations in $\mathrm{M}^{n+2}$ act within $\mathcal{C}^{n}$. The braided fusion $n$ category of all the $(n-1) \mathrm{d}$ excitations is given by $\mathcal{M}^{n}=$ $\Omega \operatorname{Hom}\left(\mathrm{M}^{n+2}, \mathrm{M}^{n+2}\right)$, which acts on $\mathcal{C}^{n}$. In other words, $\mathcal{C}^{n}$ is a left module over $\mathcal{M}^{n}$. It is also a right module over $n \mathcal{V e c}=\operatorname{Hom}\left(\left.\right|^{n+1}, \mathrm{I}^{n+1}\right)$. A gapped boundary, up to an invertible topological order, is described by a pair $\left(\mathcal{C}^{n}, \mathcal{M}^{n}\right)$. In fact, the data $\left(\mathcal{C}^{n}, \mathcal{M}^{n}\right)$ determine the boundary excitations, which in turn determine the gapped boundary, up to an invertible topological order. We believe that the data $\left(\mathcal{C}^{n}, \mathcal{M}^{n}\right)$ can determine the category of the bulk excitations (i.e., determine the bulk topological order up to an invertible topological order). In fact, we believe that $\mathcal{C}^{n}$ alone can uniquely determine the category of the bulk excitations.

Proposition 18. There is only one anomaly-free topological order $\mathrm{M}^{n+2}$ in $\mathrm{TO}_{\mathrm{af}}^{n+2}$, up to invertible topological orders, which gives rise to the category of boundary excitations:

$$
\begin{aligned}
\mathcal{C}^{n} & =\operatorname{Hom}\left(\mathrm{C}^{n+1}, \mathrm{C}^{n+1}\right), \\
\text { where } \quad \mathrm{C}^{n+1} & \in \operatorname{Hom}\left(\mathrm{I}^{n+2}, \mathrm{M}^{n+2}\right) .
\end{aligned}
$$

The above result can be rephrased. Let us denote the fusion $(n+1)$-category of the bulk excitations as $\mathcal{M}^{n+1}$ [which is given by $\operatorname{Hom}\left(\mathrm{M}^{n+2}, \mathrm{M}^{n+2}\right)$ ]. Then $\mathcal{M}^{n+1}$ is uniquely determined by a braided fusion $n$-category $\mathcal{M}^{n}=\Omega \mathcal{M}^{n+1}$, via delooping: $\mathcal{M}^{n+1}=\Sigma \mathcal{M}^{n}$, since the bulk topological order is anomaly-free.

Proposition 19. The braided fusion $n$-category $\mathcal{M}^{n}$ is uniquely determined by $\mathcal{C}^{n}$,

$$
\mathcal{M}^{n}=Z_{1}\left(\mathcal{C}^{n}\right),
$$

where boundary-bulk relation $Z_{1}$ is called $Z_{1}$ center (the Drinfeld center when $n=1$ ). Thus, $\mathcal{C}^{n}$ uniquely determines $\mathcal{M}^{n+1}$ via

$$
\mathcal{M}^{n+1}=\Sigma Z_{1}\left(\mathcal{C}^{n}\right) .
$$

Mathematically, the above result is phrased as follows:

Proposition 20. From any fusion $n$-category $\mathcal{C}^{n}$, we can always construct a unique braided fusion $n$-category $Z_{1}\left(\mathcal{C}^{n}\right)$, which is the maximal one equipped with a central monoidal functor $\mathcal{M}^{n} \stackrel{F_{\mathcal{C}^{n}}}{\longrightarrow} \mathcal{C}^{n}$, i.e., for any $\boldsymbol{x}$ in $\mathcal{M}^{n}$ and $\boldsymbol{y}$ in $\mathcal{C}^{n}$

$$
F_{\mathcal{C}^{n}}(\boldsymbol{x}) \otimes \boldsymbol{y} \simeq \boldsymbol{y} \otimes F_{\mathcal{C}^{n}}(\boldsymbol{x}),
$$

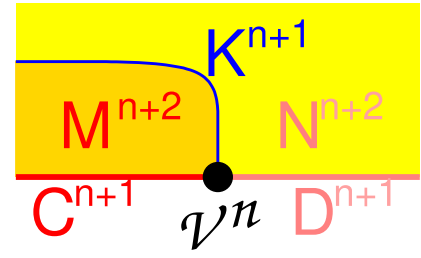

FIG. 14. The "bulk" of a domain wall on the boundary [26].

such that $Z_{1}\left(\mathcal{C}^{n}\right)$ is the category of codimension-2 excitations in the bulk of $\mathcal{C}^{n}$

$$
\mathcal{M}^{n}=Z_{1}\left(\mathcal{C}^{n}\right) .
$$

Such central functor $F_{\mathcal{C}^{n}}: Z_{1}\left(\mathcal{C}^{n}\right) \rightarrow \mathcal{C}^{n}$ is also referred to as the forgetful functor, since by construction, objects in $Z_{1}\left(\mathcal{C}^{n}\right)$ can be viewed as objects in $\mathcal{C}^{n}$ equipped with additional half-braiding structures.

We now explain an explicit construction of $Z_{1}$. To do this, consider a slightly more complicated configuration as in Fig. 14, where $\mathrm{C}^{n+1} \in \operatorname{Hom}\left(\mathrm{I}^{n+2}, \mathrm{M}^{n+2}\right)$, $\mathrm{D}^{n+1} \in \operatorname{Hom}\left(I^{n+2}, \mathrm{~N}^{n+2}\right), \quad \mathrm{K}^{n+1} \in \operatorname{Hom}\left(\mathrm{M}^{n+2}, \mathrm{~N}^{n+2}\right), \quad$ and $\mathcal{V}^{n}=\operatorname{Hom}\left(\mathrm{D}^{n+1}, \mathrm{~K}^{n+1} \underset{\mathrm{M}^{n+2}}{\otimes} \mathrm{C}^{n+1}\right)$. We view $\mathcal{V}^{n}$ as a collection of domain walls between the boundary $\mathrm{C}^{n+1}$ and $\mathrm{D}^{n+1}$ and it uniquely determines the "bulk" $\mathrm{K}^{n+1}$, which is a domain wall between the bulk of $\boldsymbol{C}^{n+1}$, and the bulk of $\mathrm{D}^{n+1}$, namely between $\mathrm{M}^{n+2}$ and $\mathrm{N}^{n+2}$.

Observe that all three fusion $n$-categories, $\mathcal{K}^{n}=$ $\operatorname{Hom}\left(\mathrm{K}^{n+1}, \mathrm{~K}^{n+1}\right), \quad \mathcal{C}^{n}=\operatorname{Hom}\left(\mathrm{C}^{n+1}, \mathrm{C}^{n+1}\right), \quad$ and $\quad \mathcal{D}^{n}=$ $\operatorname{Hom}\left(\mathrm{D}^{n+1}, \mathrm{D}^{n+1}\right)$, act on $\mathcal{V}^{n}$. Moreover, the three actions commute with each other. Here we want to separate the action of $\mathcal{K}^{n}$ from those of $\mathcal{C}^{n}$ and $\mathcal{D}^{n}$.

Let us introduce Fun $\left(\mathcal{V}^{n}, \mathcal{V}^{n}\right)$ as a collection of endofunctors of the linear $n$-category $\mathcal{V}^{n}$, or more precisely, a category of linear functors $f: \mathcal{V} \rightarrow \mathcal{V}$. In other words, for objects $\boldsymbol{v}, \boldsymbol{w} \in \mathcal{V}$, the functor $f$ satisfies

$$
f(\boldsymbol{v} \oplus \boldsymbol{w}) \simeq f(\boldsymbol{v}) \oplus f(\boldsymbol{w}) .
$$

Note that these functors are higher functors between higher categories and consist of many structures at different levels of morphisms. In this paper, we are not giving rigorous descriptions, but only listing the structures at the object level for illustration. The structures on higher morphisms are similar.

$\operatorname{Fun}\left(\mathcal{V}^{n}, \mathcal{V}^{n}\right)$ is naturally a linear monoidal category since, for $f, g \in \operatorname{Fun}\left(\mathcal{V}^{n}, \mathcal{V}^{n}\right)$, we can define

$$
(f \otimes g)(\boldsymbol{v})=f(g(\boldsymbol{v})), \quad(f \oplus g)(\boldsymbol{v})=f(\boldsymbol{v}) \oplus g(\boldsymbol{v}) .
$$

Now we can see that an action of $\mathcal{K}^{n}$ on $\mathcal{V}^{n}$ is the same as a monoidal functor $\mathcal{K}^{n} \rightarrow \operatorname{Fun}\left(\mathcal{V}^{n}, \mathcal{V}^{n}\right)$; in other words, an object $\boldsymbol{k} \in \mathcal{K}^{n}$ corresponds to a functor $f_{\boldsymbol{k}} \in \operatorname{Fun}\left(\mathcal{V}^{n}, \mathcal{V}^{n}\right)$,

$$
f_{\boldsymbol{k}}(\boldsymbol{v})=\boldsymbol{k} \underset{\mathcal{K}^{n}}{\otimes} \boldsymbol{v}, \quad \boldsymbol{v} \in \mathcal{V}^{n},
$$

where $\boldsymbol{k} \underset{\mathcal{K}^{n}}{\otimes} \boldsymbol{v}$ describes the fusion of an object $\boldsymbol{k} \in \mathcal{K}^{n}$ to an object $v \in \mathcal{V}^{n}$ along the domain wall $\mathrm{K}^{n+1}$.

Similarly, we have the actions of $\mathcal{C}^{n}$ and $\mathcal{D}^{n}$ on $\mathcal{V}^{n}$, which commute with each other and make $\mathcal{V}^{n}$ into a $\mathcal{C}^{n}-\mathcal{D}^{n}$ bimodule. Thus, the action of $\mathcal{K}^{n}$, that commutes with both actions of $\mathcal{C}^{n}$ and $\mathcal{D}^{n}$, identifies $\mathcal{K}^{n}$ with the bimodule 
endofunctors of $\mathcal{V}^{n}$, i.e., all the linear functors that commute with both actions. More precisely, if we denote the left action of $\mathcal{C}^{n}$ by $C \otimes \boldsymbol{v}$ and right action of $\mathcal{D}^{n}$ by $\boldsymbol{v} \otimes \boldsymbol{d}$ for $\boldsymbol{v} \in \mathcal{V}^{n}, \quad \mathrm{C} \in \mathcal{C}^{n},{ }^{\mathcal{C}^{n}}, \boldsymbol{d} \in \mathcal{D}^{n}$, a bimodule functor is a functor $f: \mathcal{V}^{n} \rightarrow \mathcal{V}^{n}$ together with natural isomorphisms

$$
f\left(\mathrm{C} \underset{\mathcal{C}^{n}}{\otimes} \boldsymbol{v}\right) \simeq \mathrm{C} \underset{\mathcal{C}^{n}}{\otimes} f(\boldsymbol{v}), \quad f\left(\boldsymbol{v} \underset{\mathcal{D}^{n}}{\otimes} \boldsymbol{d}\right) \simeq f(\boldsymbol{v}) \underset{\mathcal{D}^{n}}{\otimes} \boldsymbol{d},
$$

and other appropriate higher structures. We note that the above are additional structures rather than conditions. Denote the category of all bimodule functors by $\operatorname{Fun}_{\mathcal{C}^{n} \mid \mathcal{D}^{n}}\left(\mathcal{V}^{n}, \mathcal{V}^{n}\right)$. The monoidal functor $\mathcal{K}^{n} \rightarrow \operatorname{Fun}\left(\mathcal{V}^{n}, \mathcal{V}^{n}\right)$ can be promoted to $\mathcal{K}^{n} \rightarrow \operatorname{Fun}_{\mathcal{D}^{n} \mid \mathcal{C}^{n}}\left(\mathcal{V}^{n}, \mathcal{V}^{n}\right)$. Following the holographic principle, we expect such functor to be an equivalence. Thus, we have the following boundary-bulk relation, extended to domain walls on the boundary and in the bulk:

Proposition 21. Let $\quad \mathrm{C}^{n+1} \in \operatorname{Hom}\left(\mathrm{I}^{n+2}, \mathrm{M}^{n+2}\right)$, $\mathrm{D}^{n+1} \in \operatorname{Hom}\left(\mathrm{I}^{n+2}, \mathrm{~N}^{n+2}\right)$, and their excitations $\mathcal{C}^{n}=$ $\operatorname{Hom}\left(\mathrm{C}^{n+1}, \mathrm{C}^{n+1}\right), \quad \mathcal{D}^{n}=\operatorname{Hom}\left(\mathrm{D}^{n+1}, \mathrm{D}^{n+1}\right) . \quad$ A $\quad \mathcal{C}^{n}-\mathcal{D}^{n}-$ bimodule $\mathcal{V}^{n}$, viewed as a collection of domain walls between $\mathrm{C}^{n+1}$ and $\mathrm{D}^{n+1}$, uniquely determines a domain wall $\mathrm{K}^{n+1}$ in the bulk, i.e., $\mathrm{K}^{n+1} \in \operatorname{Hom}\left(\mathrm{M}^{n+2}, \mathrm{~N}^{n+2}\right)$. In other words, there is a unique $\mathrm{K}^{n+1} \in \operatorname{Hom}\left(\mathrm{M}^{n+2}, \mathrm{~N}^{n+2}\right)$ such that $\mathcal{V}^{n}=\operatorname{Hom}\left(\mathrm{D}^{n+1}, \mathrm{~K}^{n+1} \otimes_{\mathrm{M}^{n+2}} \mathrm{C}^{n+1}\right)$. The excitations on $\mathrm{K}^{n+1}$ is given by

$$
\mathcal{K}^{n}=\operatorname{Hom}\left(\mathrm{K}^{n+1}, \mathrm{~K}^{n+1}\right)=\operatorname{Fun}_{\mathcal{C}^{n} \mid \mathcal{D}^{n}}\left(\mathcal{V}^{n}, \mathcal{V}^{n}\right) .
$$

Objects in $\mathcal{K}^{n}$ correspond to functors in $\operatorname{Fun}_{\mathcal{C}^{n} \mid \mathcal{D}^{n}}\left(\mathcal{V}^{n}, \mathcal{V}^{n}\right)$.

As a special case, take $\mathcal{V}^{n}=\mathcal{D}^{n}=\mathcal{C}^{n}$; i.e., we view $\mathcal{C}^{n}$ as a collection of domain walls between $\mathrm{C}^{n+1}$ and itself. The "bulk" of $\mathcal{C}^{n}$ is the trivial domain wall in the bulk of $\mathrm{C}^{n+1}$ and the excitations on the trivial domain wall are just the codimension- 2 excitations in the bulk of $\mathrm{C}^{n+1}$. We obtain the explicit construction

$$
\mathcal{M}^{n}=Z_{1}\left(\mathcal{C}^{n}\right):=\operatorname{Fun}_{\mathcal{C}^{n} \mid \mathcal{C}^{n}}\left(\mathcal{C}^{n}, \mathcal{C}^{n}\right)
$$

For a bimodule functor $f \in Z_{1}\left(\mathcal{C}^{n}\right)$, and any $\boldsymbol{y} \in \mathcal{C}^{n}$

$$
f\left(\mathbf{1}_{\mathcal{C}^{n}}\right) \otimes \boldsymbol{y} \simeq f(\boldsymbol{y}) \simeq \boldsymbol{y} \otimes f\left(\mathbf{1}_{\mathcal{C}^{n}}\right) .
$$

We see that a bimodule funcor $f$ is the same as an object $f\left(\mathbf{1}_{\mathcal{C}^{n}}\right)$ in $\mathcal{C}^{n}$ together with the half-braiding $f\left(\mathbf{1}_{\mathcal{C}^{n}}\right) \otimes \boldsymbol{y} \simeq$ $\boldsymbol{y} \otimes f\left(\mathbf{1}_{\mathcal{C}^{n}}\right)$. The forgetful functor is thus

$$
\begin{aligned}
F_{\mathcal{C}^{n}}: \operatorname{Fun}_{\mathcal{C}^{n} \mid \mathcal{C}^{n}}\left(\mathcal{C}^{n}, \mathcal{C}^{n}\right) & =Z_{1}\left(\mathcal{C}^{n}\right) \rightarrow \mathcal{C}^{n}, \\
f & \mapsto F_{\mathcal{C}^{n}}(f)=f\left(\mathbf{1}_{\mathcal{C}^{n}}\right)
\end{aligned}
$$

In this paper, we mainly use the third version of boundarybulk relation $Z_{1}\left(\mathcal{C}^{n}\right)=\mathcal{M}^{n}$ : The codimension-1 boundary excitations described by a fusion $n$-category $\mathcal{C}^{n}$ uniquely determine the codimension- 2 bulk excitations described by a braided fusion $n$-category $\mathcal{M}^{n}$. In contrast, (41) is a relation between a boundary topological order [i.e., an anomalous $n \mathrm{~d}$ topological order-an object in $\left.\operatorname{Hom}\left(\mathrm{I}^{n+2}, \mathrm{M}^{n+2}\right)\right]$ and a bulk topological order (i.e., an anomaly-free $(n+1)$ d topological order-an object in $\mathrm{TO}_{\mathrm{af}}^{n+2}$ ).

\section{J. Example of topological orders and the corresponding higher categories \\ 1. Invertible topological orders}

The simplest anomaly-free topological orders are invertible topological orders $[25,41,42]$. We use $\mathcal{T O}_{\text {inv }}^{n+1}$ to denote the category of all $n$ d invertible topological orders. We believe that there are no anomalous invertible topological orders:

Proposition 22. Consider a potentially anomalous topological order in $n$-dimensional space: $\mathrm{C}^{n+1} \in$ $\operatorname{Hom}\left(\mathrm{I}^{n+2}, \mathrm{M}^{n+2}\right)$ for $\mathrm{M}^{n+2} \in \mathbb{T O}_{\mathrm{af}}^{n+2}$, if its excitations are the same as those for the trivial topological order, i.e., $\Omega \mathrm{C}^{n+1}=\Omega \mathrm{I}^{n+1}=n \mathcal{V e c}$, then $\mathrm{M}^{n+2}=\mathrm{I}^{n+2}$ (i.e., $\mathrm{C}^{n+1}$ is anomaly-free) and $\mathrm{C}^{n+1}$ is an invertible topological order.

By definition, the invertible topological orders form Abelian groups under the stacking $\otimes$. In different dimensions, those groups are given by $[25,41,42]$

$$
\begin{array}{cccccc}
(n+1) \mathrm{D}: & 0+1 & 1+1 & 2+1 & 3+1 & 4+1 \\
\mathcal{T O}_{\text {inv }}^{n+1}: & 0 & 0 & \mathbb{Z} & 0 & \mathbb{Z}_{2}
\end{array} .
$$

The generator of $\mathcal{T O}_{\text {inv }}^{3}$ is the $E_{8}$ bosonic quantum Hall state described by the wave function

$$
\Psi\left(z_{i}^{I}\right)=\left[\prod_{i, j} \prod_{I<J}\left(z_{i}^{I}-z_{j}^{J}\right)^{K_{I J}}\right]\left[\prod_{i<j} \prod_{I}\left(z_{i}^{I}-z_{j}^{I}\right)^{K_{I I}}\right] e^{-\frac{1}{4} \sum_{i, I}\left|z_{i}^{I}\right|^{2}},
$$

where the $K$-matrix is given by

$$
K=\left(\begin{array}{llllllll}
2 & 1 & 0 & 0 & 0 & 0 & 0 & 0 \\
1 & 2 & 1 & 0 & 0 & 0 & 1 & 0 \\
0 & 1 & 2 & 1 & 0 & 0 & 0 & 0 \\
0 & 0 & 1 & 2 & 1 & 0 & 0 & 0 \\
0 & 0 & 0 & 1 & 2 & 1 & 0 & 0 \\
0 & 0 & 0 & 0 & 1 & 2 & 0 & 0 \\
0 & 1 & 0 & 0 & 0 & 0 & 2 & 1 \\
0 & 0 & 0 & 0 & 0 & 0 & 1 & 2
\end{array}\right),
$$

which satisfies $\operatorname{det}(K)=1$. The generator of $\mathcal{T O}_{\text {inv }}^{5}$ is given following $4 \mathrm{~d}$ bosonic system (described by a path integral for cochain fields [85])

$$
Z=\sum_{a^{\mathbb{Z}_{2}}, b^{\mathbb{Z}_{2}}} e^{i \pi \int_{M^{4+1}}\left(\mathrm{w}_{2}+d a^{\mathbb{Z}_{2}}\right)\left(\mathrm{w}_{3}+d b^{\mathbb{Z}_{2}}\right)},
$$

where $a^{\mathbb{Z}_{2}}$ is a $\mathbb{Z}_{2}$-valued 1-cochain, $b^{\mathbb{Z}_{2}}$ is a $\mathbb{Z}_{2}$-valued 2cochain, and $\mathrm{w}_{n}$ is the $n$th Stiefel-Whitney class of the tangent bundle of the closed spacetime manifold $M^{4+1}$. The path integral only depends on the cohomology classes of $\mathrm{w}_{2}$ and $\mathrm{w}_{3}$, since the path integral is invariant under the following gauge transformation:

$$
\begin{aligned}
& \mathrm{w}_{2} \rightarrow \mathrm{w}_{2}+d \gamma, \quad \mathrm{w}_{3} \rightarrow \mathrm{w}_{3}+d \lambda, \\
& a^{\mathbb{Z}_{2}} \rightarrow a^{\mathbb{Z}_{2}}+\gamma, \quad b^{\mathbb{Z}_{2}} \rightarrow b^{\mathbb{Z}_{2}}+\lambda .
\end{aligned}
$$

The path integral can be calculated exactly

$$
\begin{aligned}
Z & =\sum_{a^{\mathbb{Z}_{2}, b^{\mathbb{Z}}}} e^{i \pi \int_{M^{4+1}}\left(\mathrm{w}_{2}+d a^{\mathbb{Z}_{2}}\right)\left(\mathrm{w}_{3}+d b^{\mathbb{Z}_{2}}\right)}, \\
& =2^{N_{l}+N_{t}} d^{i \pi \int_{M^{4+1} \mathrm{w}_{2} \mathrm{w}_{3}},}
\end{aligned}
$$


where $N_{l}$ is the number of the links and $N_{t}$ is the number of the triangles in the triangulated spacetime $M^{4+1}$. The nontrivial topological invariant $e^{i \pi \int_{M^{4+1}} \mathrm{w}_{2} \mathrm{w}_{3}}$ implies that (60) realizes a nontrivial $4 \mathrm{~d}$ invertible topological order.

The invertible topological order have no nontrivial nl-type of excitations, i.e., no nontrivial topological excitations. All the excisions are local. The different types of local excitations are described by the trivial fusion $n$-category $n \mathcal{V e c}$ for an $n \mathrm{~d}$ invertible topological order.

For example, in 2-dimensional space, the objects in the category of invertible topological orders $\mathcal{T O}_{\text {inv }}^{3}$ form an Abelian group $\mathbb{Z}$. The morphisms on each object form a trivial fusion 2-category $2 \mathcal{V}$ ec. Since the $E_{8}$ quantum Hall state has no gapped boundary, it is not an exact topological order, but is a closed (i.e., anomaly-free) topological order. Therefore, $\mathcal{T O}_{\text {inv }}^{3}$ has no 1-morphisms between different objects. All domain walls between different objects are gapless. The 1-morphism that connects the same object is also trivial. This is because such a 1-morphism corresponds to an $1+1 \mathrm{D}$ excitation and there is no nontrivial $1+1 \mathrm{D}$ anomaly-free topological order.

In our attempt to use higher categories to characterize topological orders, the invertible topological orders are the most difficult ones. This is because higher categories mainly describe the excitations, but the excitations on top of invertible topological orders are identical to those on top of of trivial product state. Fortunately, in the category of topological orders, we also have information on the stacking operation $\otimes$ and the gapped domain walls between topological orders. This allows us to distinguish invertible topological orders. The invertible topological orders in $2 \mathrm{~d}$ are particularly difficult, since we do not even have any gapped domain walls (i.e., no 1-morphisms). Only the stacking operation $\otimes$ allows us to distinguish $2 \mathrm{~d}$ invertible topological orders.

\section{G-topological orders}

Another class of topological orders for bosonic systems are called $G$-topological orders (see Sec. IV), which are described by gauge theories with a finite group $G$. We use $\mathrm{GT}_{G}^{n+1} \in$ $\mathrm{TO}_{\text {af }}^{n+1}$ to denote $G$-topological order in $n$-dimensional space. We use $\Omega \mathrm{GT}_{G}^{n+1}$ to denote the fusion $n$-category that describes the excitations in $\mathrm{GT}_{G}^{n+1}$ and use $\Omega^{2} \mathrm{GT}_{G}^{n+1}$ to denote the braided fusion $(n-1)$-category that describes the excitations with codimension-2 and higher in $\mathrm{GT}_{G}^{n+1}$. It is known that $\mathrm{GT}_{G}^{n+1}$ is anomaly-free and has gapped boundary. An example of $\mathrm{GT}_{\mathbb{Z}_{2}}^{3}$ is given in Example 1 .

Let us describe the $3 \mathrm{~d} \mathbb{Z}_{2}$-topological order $\mathrm{GT}_{\mathbb{Z}_{2}}^{4}$ in more detail. Such a state has two nl-types of point-like excitations $\mathbf{1}, e$, two nl-types of string-like excitation $\mathbf{1}_{s}, m_{s}$, and one trivial nl-type membrane-like excitations. The $e$-particle has a fusion $e \otimes e=\mathbf{1}$ and the $m_{s}$-loop has a fusion $m_{s} \otimes m_{s}=$ $\mathbf{1}_{s}$. The $\mathbb{Z}_{2}$-topological order also has two types of pointlike excitations $\mathbf{1}, e$, and four types of string-like excitation $\mathbf{1}_{s}, m_{s}, e_{s}, e_{s} \otimes m_{s}$. The string $e_{s}$ is formed by $e$-particles condensing into the $\mathbb{Z}_{2}$ symmetry-breaking state. The $e_{s}$-loop has a fusion $e_{s} \otimes e_{s}=2 e_{s}$. Those point-like and string-like excitations form the braided fusion 2-category $\Omega^{2} \mathrm{GT}_{\mathbb{Z}_{2}}^{4}$.

The $3 \mathrm{~d} \mathbb{Z}_{2}$-topological order $\mathrm{GT}_{\mathbb{Z}_{2}}^{4}$ has infinite types of membrane-like excitations corresponding to infinite different
$2 \mathrm{~d}$ topological orders formed by trivial point-like excitations 1's. $\mathrm{GT}_{\mathbb{Z}_{2}}^{4}$ also has infinite types of membrane-like excitations corresponding to infinite different $2 \mathrm{~d}$ SET orders with $\mathbb{Z}_{2}$ symmetry, formed by $e$-particles with mod- 2 conservation. There are three types of membrane-like excitations corresponding to $2 \mathrm{~d}$ topological orders formed in $m_{s}$-loops. The $m_{s}$-loops have a mod- 2 conservation that corresponds to a $\mathbb{Z}_{2}$ higher symmetry. Thus, this kind of $2 \mathrm{~d}$ topological order can be viewed as having a spontaneous breaking of $\mathbb{Z}_{2} 1$-symmetry. Those point-like, string-like, and membrane-like excitations form the fusion 3-category $\Omega \mathrm{GT}_{\mathbb{Z}_{2}}^{4}$. The point-like and stringlike excitations form the braided fusion 2-category $\Omega^{2} G T_{\mathbb{Z}_{2}}^{4}$.

The above $3 \mathrm{~d} \mathbb{Z}_{2}$-topological order $\mathrm{GT}_{\mathbb{Z}_{2}}^{4}$ is anomaly-free, which means that it can be realized by a bosonic lattice model, as shown in Sec. IV. Another way to realize $\mathrm{GT}_{\mathbb{Z}_{2}}^{4}$ is via the path integral of $\mathbb{Z}_{2}$-valued 1-cochain fields, $a^{\mathbb{Z}_{2}}$ [85]:

$$
Z=\sum_{d a^{\mathbb{Z}_{2}=0}} 1,
$$

where $\sum_{\mathrm{d} a \mathbb{Z}_{2}=0}$ is a summation over $\mathbb{Z}_{2}$-valued 1-cocycles. One can also realize $\mathrm{GT}_{\mathbb{Z}_{2}}^{4}$ via the path integral of $\mathbb{Z}_{2}$-valued 2-cochain fields, $b^{\mathbb{Z}_{2}}[85]$ :

$$
Z=\sum_{d b^{\mathbb{Z}_{2}}=0} 1
$$

where $\sum_{\mathrm{d} b \mathbb{Z}_{2}=0}$ is a summation over $\mathbb{Z}_{2}$-valued 2-cocycles.

Since $\mathrm{GT}_{\mathbb{Z}_{2}}^{4}$ is anomaly-free, its excitations described by $\Omega \mathrm{GT}_{\mathbb{Z}_{2}}^{4}$ satisfy

$$
Z_{1}\left(\Omega \mathrm{GT}_{\mathbb{Z}_{2}}^{4}\right)=\Omega 4 \mathcal{V e c} \equiv 3 \mathcal{V e c} .
$$

The above boundary-bulk relation between fusion higher categories and braided fusion higher categories only tell us that $\mathrm{GT}_{\mathbb{Z}_{2}}^{4}$ is either anomaly-free or has invertible anomaly. The stronger boundary-bulk relation is given by

$$
\operatorname{Bulk}\left(\mathrm{GT}_{\mathbb{Z}_{2}}^{4}\right)=\mathrm{I}^{5}
$$

This boundary-bulk relation tells us that $\mathrm{GT}_{\mathbb{Z}_{2}}^{4}$ is anomalyfree.

We would like to mention that there is also a $3 \mathrm{~d}$ twisted $\mathbb{Z}_{2}$-topological order where the point-like $\mathbb{Z}_{2}$-charges are fermions. We denote such a twisted $\mathbb{Z}_{2}$-topological order as $\mathrm{GT}_{\mathbb{Z}_{2}^{f}}^{4}$. The twisted $\mathbb{Z}_{2}$-topological order $\mathrm{GT}_{\mathbb{Z}_{2}^{f}}^{4}$ is also anomaly-free and can be realized by the path integral of $\mathbb{Z}_{2^{-}}$ valued 2 -cochain fields, $b^{\mathbb{Z}_{2}}[56,57,85]$ :

$$
Z=\sum_{d b^{\mathbb{Z}_{2}}=0} d^{i \pi \int_{M^{3+1}} b^{\mathbb{Z}_{2}} b^{\mathbb{Z}_{2}}}=\sum_{d b^{\mathbb{Z}_{2}}=0} e^{i \pi \int_{M^{3+1}} b^{\mathbb{Z}_{2}} \mathrm{w}_{2}},
$$

where $\sum_{\mathrm{d} b \mathbb{Z}_{2}=0}$ is a summation over $\mathbb{Z}_{2}$-valued 2-cocycles, $M^{3+1}$ is a $(3+1)$-dimensional closed spacetime (with a triangulation), and $\mathrm{w}_{2}$ is the second Stiefel-Whitney class of the tangent bundle of $M^{3+1}$. Here we used a fact that $b^{\mathbb{Z}_{2}} b^{\mathbb{Z}_{2}}+b^{\mathbb{Z}_{2}} \mathrm{~W}_{2}$ is a $\mathbb{Z}_{2}$-valued coboundary. The topological term $e^{i \pi \int_{M^{3+1}} b^{\mathbb{Z}_{2}} b^{\mathbb{Z}_{2}}}=e^{i \pi \int_{M^{3+1}} b^{\mathbb{Z}_{2} \mathrm{w}_{2}}}$ makes the point-like $\mathbb{Z}_{2^{-}}$ charges to be fermions. 


\section{A 2d anomalous topological order}

Now, let us consider an anomalous topological order in $2 \mathrm{~d}$, denoted as $\mathrm{C}_{\mathrm{Z}_{2}}^{3}$, which has two nl-types of point-like excitations, labeled by $\mathbf{1}, e$, where $\mathbf{1}$ is a trivial point-like excitation and $e$ has a $\mathbb{Z}_{2}$ fusion $e \otimes e=\mathbf{1}$. The anomalous topological order has two types of point-like excitations, which are also given by $\mathbf{1}, e$. The anomalous topological order has only one nl-type of string-like excitations, which is a local string-like excitation. But it has two types of string-like excitations, labeled by $\mathbf{1}_{s}, e_{s}$. The $e_{s}$-type of string-like excitation is formed by the $e$-particles, condensing into a $1 \mathrm{~d}$ phase of spontaneous $\mathbb{Z}_{2}$ symmetry-breaking state. The $e_{s}$ loop has a fusion $e_{s} \otimes e_{s}=2 e_{s} . \mathbf{1}_{s}, e_{s}$ are local string-like excitations, i.e., they belong to the trivial nl-type of string-like excitations.

The excitations in the anomalous topological order $\mathrm{C}_{\mathbb{Z}_{2}}^{3}$ are described by a fusion 2-category $\mathcal{C}_{\mathbb{Z}_{2}}^{2}=\Omega C_{\mathbb{Z}_{2}}^{3}=2 \mathcal{R e p}\left(\mathbb{Z}_{2}\right)$. $\mathcal{C}_{\mathbb{Z}_{2}}^{2}$ has two simple objects $\mathbf{1}_{s}, e_{s}$. On $\mathbf{1}_{s}$, there are two simple 1-morphisms $\mathbf{1}, e$. On $e_{s}$, there are also two simple 1-morphisms $\mathbf{1}_{e_{s}}, d_{e_{s}}$, with a fusion rule $d_{e_{s}} \otimes d_{e_{s}}=\mathbf{1}_{e_{s}}$. There is one simple 1-morphism $\sigma \in \operatorname{Hom}\left(\mathbf{1}_{s}, e_{s}\right)$ and one simple $\bar{\sigma} \in \operatorname{Hom}\left(e_{s}, \mathbf{1}_{s}\right)$, with fusion rules

$$
\begin{aligned}
& \sigma \underset{\mathbf{1}_{s}}{\otimes \mathbf{1}}=\sigma \underset{\mathbf{1}_{s}}{\otimes} e=\mathbf{1}_{e_{s}} \underset{e_{s}}{\otimes} \sigma=d_{e_{s}} \underset{e_{s}}{\otimes} \sigma=\sigma, \\
& \mathbf{1} \underset{\mathbf{1}_{s}}{\otimes} \bar{\sigma}=e \underset{\mathbf{1}_{s}}{\otimes} \bar{\sigma}=\bar{\sigma} \underset{e_{s}}{\otimes} \mathbf{1}_{e_{s}}=\bar{\sigma} \underset{e_{s}}{\otimes} d_{e_{s}}=\bar{\sigma}, \\
& \bar{\sigma} \otimes \sigma=\mathbf{1} \oplus e, \\
& e_{s} \\
& \sigma \underset{\mathbf{1}_{s}}{\otimes} \bar{\sigma}=\mathbf{1}_{e_{s}} \oplus d_{e_{s}}
\end{aligned}
$$

The bulk of the anomalous topological order $C_{\mathbb{Z}_{2}}^{3}$ is the $\mathbb{Z}_{2-}$ topological order in 3-dimensional space $\mathrm{GT}_{\mathbb{Z}_{2}}^{4}$ :

$$
\operatorname{Bulk}\left(\mathrm{C}_{\mathbb{Z}_{2}}^{3}\right)=\mathrm{GT}_{\mathbb{Z}_{2}}^{4} .
$$

Since $\mathrm{GT}_{\mathbb{Z}_{2}}^{4}$ is nontrivial, $\mathrm{C}_{\mathbb{Z}_{2}}^{3}$ is anomalous. In fact, $\mathrm{C}_{\mathbb{Z}_{2}}^{3}$ is a $2 \mathrm{~d}$ gapped boundary of the $3 \mathrm{~d} \mathbb{Z}_{2}$ topological order $\mathrm{GT}_{\mathbb{Z}_{2}}^{4}$ obtained via condensation of $Z_{2}$-flux strings. We have a similar relation for excitations

$$
Z_{1}\left(\mathcal{C}_{\mathbb{Z}_{2}}^{2}\right)=\Omega^{2} \mathrm{GT}_{\mathbb{Z}_{2}}^{4}
$$

where $\mathcal{C}_{\mathbb{Z}_{2}}^{2}=\Omega C_{\mathbb{Z}_{2}}^{3}$ is the fusion 2-category describing the excitations in $C_{\mathbb{Z}_{2}}^{3}$. The relation (69) carries more information than (70). We would like to remark that when we stack the two anomalous topological orders, both the boundaries and the bulks are stacked:

$$
\operatorname{Bulk}\left(\mathrm{C}_{\mathbb{Z}_{2}}^{3} \otimes \mathrm{C}_{\mathbb{Z}_{2}}^{3}\right)=\mathrm{GT}_{\mathbb{Z}_{2}}^{4} \otimes \mathrm{GT}_{\mathbb{Z}_{2}}^{4} .
$$

\section{Anomalous $3 d \mathbb{Z}_{2}$ topological order}

The anomaly-free $3 \mathrm{~d} \mathbb{Z}_{2}$-topological order $\mathrm{GT}_{\mathbb{Z}_{2}}^{4}$ discussed above can also be realized via the path integral of $\mathbb{Z}_{2}$-valued 1 -cochain and 2-cochain fields, $a^{\mathbb{Z}_{2}}$ and $b^{\mathbb{Z}_{2}}$ [85]:

$$
Z=\sum_{a^{\mathbb{Z}_{2}, b^{\mathbb{Z}_{2}}}} e^{i \pi \int_{M^{3+1}} b^{\mathbb{Z}_{2}} d a^{\mathbb{Z}_{2}}},
$$

where $\sum_{a \mathbb{Z}_{2}, \mathbb{Z}_{2}}$ is a summation over $\mathbb{Z}_{2}$-valued 1-cochain and 2 -cochain. The above path integral has a gauge invariance for closed $M^{3+1}$ :

$$
a^{\mathbb{Z}_{2}} \rightarrow a^{\mathbb{Z}_{2}}+d \alpha, \quad b^{\mathbb{Z}_{2}} \rightarrow b^{\mathbb{Z}_{2}}+d \beta .
$$

In this formulation, the twisted $3 \mathrm{~d} \mathbb{Z}_{2}$-topological order $\mathrm{GT}_{\mathbb{Z}_{2}^{f}}^{4}$ is realized by the path integral

$$
Z=\sum_{a^{\mathbb{Z}_{2}, b^{\mathbb{Z}}}} d^{i \pi \int_{M^{3+1}} b^{\mathbb{Z}_{2}} \mathrm{~d} a^{\mathbb{Z}_{2}}+b^{\mathbb{Z}_{2}} \mathrm{w}_{2}} .
$$

The above path integral is also gauge invariant for closed $M^{3+1}$.

$$
a^{\mathbb{Z}_{2}} \rightarrow a^{\mathbb{Z}_{2}}+\gamma, b^{\mathbb{Z}_{2}} \rightarrow b^{\mathbb{Z}_{2}}+d \beta, \mathrm{w}_{2} \rightarrow \mathrm{w}_{2}+d \gamma
$$

The path integral only depends on the cohomology classes of $\mathrm{w}_{2}$, so it describes an anomaly-free theory.

In this section, we are going to study an anomalous $3 \mathrm{~d} \mathbb{Z}_{2}$ topological order, realized by the following path integral:

$$
Z=\sum_{a^{\mathbb{Z}_{2}, b^{\mathbb{Z}}}} e^{i \pi \int_{M^{3+1}} b^{\mathbb{Z}_{2}} d a^{\mathbb{Z}_{2}}+a^{\mathbb{Z}_{2}} \mathrm{w}_{3}+b^{\mathbb{Z}_{2} \mathrm{w}_{2}}} .
$$

Under the gauge transformation,

$$
\begin{aligned}
& a^{\mathbb{Z}_{2}} \rightarrow a^{\mathbb{Z}_{2}}+\gamma, \quad b^{\mathbb{Z}_{2}} \rightarrow b^{\mathbb{Z}_{2}}+\lambda, \\
& \mathrm{w}_{2} \rightarrow \mathrm{w}_{2}+d \gamma, \quad \mathrm{w}_{3} \rightarrow \mathrm{w}_{3}+d \lambda,
\end{aligned}
$$

the above path integral is not invariant. The gauge noninvariance can be fixed by adding a bulk term $\mathrm{e}^{\mathrm{i} \pi \int_{N^{5}} \mathrm{w}_{2} \mathrm{w}_{3}}$ in one higher dimension, where $\partial N^{5}=M^{3+1}$. The resulting path integral

$$
Z=\sum_{a^{\mathbb{Z}_{2}}, b^{\mathbb{Z}_{2}}} d^{i \pi \int_{M^{3+1}} b^{\mathbb{Z}_{2}} d a^{\mathbb{Z}_{2}}+a^{\mathbb{Z}_{2}} \mathrm{w}_{3}+b^{\mathbb{Z}_{2}} \mathrm{w}_{2}} e^{i \pi \int_{N^{5}} \mathrm{w}_{2} \mathrm{w}_{3}}
$$

is gauge invariant; i.e., it only depends on the cohomology classes of $w_{2}$ and $w_{3}$. Since the path integral requires a bulk in one higher dimension to be gauge invariant (i.e., only depends on the cohomology classes of $\mathrm{w}_{2}$ and $\mathrm{w}_{3}$ ), so it describes an anomalous theory. We denote such a $3 \mathrm{~d}$ anomalous $\mathbb{Z}_{2}$ topological order as $\mathrm{GT}_{\mathbb{Z}_{2}^{f}}^{4, \mathrm{w}_{2} \mathrm{w}_{3}}$.

Such a $3 \mathrm{~d}$ anomalous $\mathbb{Z}_{2}$-topological order $\mathrm{GT}_{\mathbb{Z}_{2}^{f}}^{4, \mathrm{w}_{2} \mathrm{w}_{3}}$ has a fermionic point-like $\mathbb{Z}_{2}$ charge. If the world sheet for the $\mathbb{Z}_{2}$ flux loop is unorientable, there is a world line that marks the reversal of the orientation. Such an orientation-reversal world line corresponds to a fermion world line. In other words, the anomalous $\mathbb{Z}_{2}$ topological order has a special property that a unorientable world sheet of the $\mathbb{Z}_{2}$-flux must bind with a world line of the fermionic point-like $\mathbb{Z}_{2}$ charge. Such a fermionic world line corresponds to the orientation reversal loop on the unorientable world sheet.

The $3 \mathrm{~d}$ anomalous $\mathbb{Z}_{2}$-topological order $\mathrm{GT}_{\mathbb{Z}_{2}^{f}}^{4, \mathrm{w}_{2} \mathrm{w}_{3}}$ has a nontrivial bulk. The boundary-bulk relation can be written as

$$
\operatorname{Bulk}\left(\mathrm{GT}_{\mathbb{Z}_{2}^{f}}^{4, \mathrm{w}_{2} \mathrm{w}_{3}}\right)=\mathrm{I}_{\mathrm{w}_{2} \mathrm{w}_{3}}^{5},
$$

where $\mathrm{I}_{\mathrm{w}_{2} \mathrm{w}_{3}}^{5}$ is the $4 \mathrm{~d}$ invertible topological order characterized by the topological invariant $e^{i \pi \int_{N^{5}} \mathrm{w}_{2} \mathrm{w}_{3}}[25,41,42]$. The boundary-bulk relation (79) implies the following 
boundary-bulk relation for the excitations:

$$
\begin{aligned}
Z_{1}\left(\Omega \mathrm{GT}_{\mathbb{Z}_{2}^{f}}^{4, \mathrm{w}_{2} \mathrm{w}_{3}}\right) & =3 V_{\mathrm{ec}}, \\
3 \mathcal{V e c} & =\left.\Omega\right|_{\mathrm{w}_{2} \mathrm{w}_{3}} ^{5},
\end{aligned}
$$

since the excitations in an invertible topological order are described by a trivial braided fusion higher category. Although the right-hand side of $Z_{1}\left(\Omega G^{4, \mathrm{w}_{2} \mathrm{w}_{3}}\right)=3 \mathrm{Vec}$ is a trivial braided fusion higher category, as we have mentioned before, the boundary-bulk relation for fusion higher categories $Z_{1}\left(\Omega \mathrm{GT}_{\mathbb{Z}_{2}^{f}}^{4, \mathrm{w}_{2} \mathrm{w}_{3}}\right)=3$ Vec does not imply $\mathrm{GT}_{\mathbb{Z}_{2}^{f}}^{4, \mathrm{w}_{2} \mathrm{w}_{3}}$ to be anomaly-free. In fact, $\mathrm{GT}_{\mathbb{Z}_{2}^{f}}^{4, \mathrm{w}_{2} \mathrm{w}_{3}}$ has an invertible anomaly, which is a $\mathbb{Z}_{2}$ global gravitational anomaly. So (79) carries more information, which indicates that $\mathrm{GT}_{\mathbb{Z}_{2}^{f}}^{4, \mathrm{w}_{2} \mathrm{w}_{3}}$ is anomalous.

\section{AN EXAMPLE OF ALGEBRAIC HIGHER SYMMETRIES: $G$-GAUGE THEORY}

For a quantum system with usual symmetry, the Hamiltonian commutes with a set of operators which form a group under the operator product. In this section, we construct an example, in which the Hamiltonian commutes with a set of operators that do not form a group under the operator product. The constructed model is an exactly solvable $3 \mathrm{~d}$ local bosonic model [5] whose ground state has a topological order described by a $3 \mathrm{~d}$ gauge theory of a finite group $G$. The operators that commute with the Hamiltonian are the Wilson line operators. When $G$ is non-Abelian, the Wilson line operators, under the operator product, form an algebra, which is not a group.

Our lattice bosonic model is defined on a $3 \mathrm{~d}$ spatial lattice whose sites are labeled by $i$. Physical degrees of freedom live on the links which are labeled by $i j$. On an oriented link $i j$, the degrees of freedom are labeled by $g_{i j} \in G$. The labels $g_{i j}$ 's on links with opposite orientations satisfy

$$
g_{i j}=g_{j i}^{-1} .
$$

The many-body Hilbert space of our lattice bosonic model has the following local basis:

$$
\left|\left\{g_{i j}\right\}\right\rangle, \quad g_{i j} \in G, \quad i j \in \text { links of cubic lattice. }
$$

The Hamiltonian of the exactly solvable model is expressed in terms of string operators and point operators.

\section{A. The string operators}

The string operators $B_{q}\left(S^{1}\right)$ are defined on a closed loop $S^{1}$ formed by the links of the cubic lattice and are labeled by $q$, the irreducible representation of the gauge group $G$ :

$$
R_{q}\left(h_{i} g_{i j} h_{j}^{-1}\right)=R_{q}\left(h_{i}\right) R_{q}\left(g_{i j}\right) R_{q}^{-1}\left(h_{j}\right)
$$

where $R_{q}\left(g_{i j}\right)$ is the matrix of the irreducible representation. A $q$-string operator is given by

$$
B_{q}\left(S^{1}\right)\left|\left\{g_{i j}\right\}\right\rangle=\operatorname{Tr}\left[\prod_{i j \in S^{1}} R_{q}\left(g_{i j}\right)\right]\left|\left\{g_{i j}\right\}\right\rangle .
$$

So $B_{q}\left(S^{1}\right)$ is diagonal in the basis $\left|\left\{g_{i j}\right\}\right\rangle: B_{q}\left(S^{1}\right)=$ $\operatorname{Tr}\left[\prod_{i j \in S^{1}} R_{q}\left(g_{i j}\right)\right]$. We note that

$$
B_{q}\left(S^{1}\right) B_{s}\left(S^{1}\right)=\operatorname{Tr} \prod_{I \in S^{1}} R_{q}\left(g_{i j}\right) \otimes_{\mathbb{C}} R_{s}\left(g_{i j}\right) .
$$

(We use $\otimes_{\mathbb{C}}$ to denote the usual tensor product of matrices or vector spaces over the complex numbers $\mathbb{C}$, while $\otimes$ to denote the fusion of excitations.) Using

$$
R_{q} \otimes_{\mathbb{C}} R_{s}=\bigoplus_{t} N_{t}^{q s} R_{t}, \quad N_{t}^{q s} \in \mathbb{N},
$$

we see that

$$
B_{q}\left(S^{1}\right) B_{s}\left(S^{1}\right)=\sum_{t} N_{t}^{q s} B_{t}\left(S^{1}\right) .
$$

The ends of the strings are point-like topological excitations and the above $N_{t}^{q s}$ are the fusion coefficients of those topological excitations. The quantum dimensions of those topological excitations, i.e., $d_{q}=\operatorname{dim}\left(R_{q}\right)$, satisfy the following identity:

$$
\sum_{s} N_{t}^{q s} d_{s}=d_{q} d_{t}
$$

We see that these string operators form a fusion algebra which is not a group when $G$ is non-Abelian.

Let

$$
B\left(S^{1}\right)=\sum_{q} \frac{d_{q}}{D^{2}} B_{q}\left(S^{1}\right), \quad D^{2}=\sum_{q} d_{q}^{2} .
$$

We have

$$
\begin{aligned}
B^{2} & =\sum_{q, s} \frac{d_{q} d_{s}}{D^{4}} B_{q} B_{s}=\sum_{q, s, t} \frac{d_{q} d_{s}}{D^{4}} N_{t}^{q s} B_{t} \\
& =\sum_{q, t} \frac{d_{q} d_{q}}{D^{4}} d_{t} B_{t}=B .
\end{aligned}
$$

Thus, $B$ is a projection operator. In fact, it is a projection operator into the subspace with $\prod_{i j \in \text { loop }} g_{i j}=1$.

\section{B. The point operators}

A point operator is given by its action on the basis:

$$
Q_{h}(i)\left|\left\{\ldots, g_{i j}, g_{i j^{\prime}}, \ldots\right\}\right\rangle=\left|\left\{\ldots, h g_{i j}, h g_{i j^{\prime}}, \ldots\right\}\right\rangle \text {. }
$$

Clearly they satisfy

$$
Q_{h}(i) Q_{h^{\prime}}(i)=Q_{h h^{\prime}}(i) .
$$

So for a non-Abelian group $G$, in general

but we have

$$
Q_{h}(i) Q_{h^{\prime}}(i) \neq Q_{h^{\prime}}(i) Q_{h}(i),
$$

$$
Q_{h}(i) Q_{h^{\prime}}(j)=Q_{h^{\prime}}(j) Q_{h}(i), \quad i \neq j .
$$

Let us introduce

$$
C_{a}(i)=\sum_{h \in \chi_{a}} Q_{h}(i)
$$

where $\chi_{a}$ is a conjugacy class labeled by $a$. We find that

$$
C_{a}(i) C_{b}(j)=C_{b}(j) C_{a}(i)
$$

regardless if $i=j$ or not. 
We note that, on a given site $i$,

$$
C_{a}(i) C_{b}(i)=\sum_{h \in \chi_{a}} \sum_{h^{\prime} \in \chi_{b}} Q_{h h^{\prime}}(i)=\sum_{c} M_{c}^{a b} C_{c}(i) .
$$

The above expression allows us to see that $M_{c}^{a b}$ are non-negative integers. Using $C_{a} C_{b}=C_{b} C_{a}$ and $\left(C_{a} C_{b}\right) C_{c}=$ $C_{a}\left(C_{b} C_{c}\right)$, we find that

$$
M_{c}^{a b}=M_{c}^{b a}, \quad \sum_{d} M_{d}^{a b} M_{e}^{d c}=\sum_{d} M_{e}^{a d} M_{d}^{b c} .
$$

Let $\left(M_{a}\right)_{c b}=M_{c}^{a b}$, and we can rewrite the second equation in the above as

$$
M_{c} M_{a}=M_{a} M_{c} .
$$

For example, the permutation group of three elements $S_{3}=\{(123),(132),(321),(213),(231),(312)\}$ has three conjugacy classes: $\chi_{1}=\{(123)\}, \chi_{2}=\{(132),(321),(213)\}$, and $\chi_{3}=\{(231),(312)\}$. We find that

$$
\begin{aligned}
& C_{1} C_{a}=C_{a}, \quad C_{2} C_{2}=3 C_{1}+3 C_{3}, \\
& C_{3} C_{3}=2 C_{1}+C_{3}, \quad C_{2} C_{3}=2 C_{2} .
\end{aligned}
$$

Let $C$ be a particular common eigenvector of $M_{a}$ whose components are all non-negative. (Such common eigenvector exists since the matrix elements of $M_{a}$ are all non-negative.) The eigenvalue of such an eigenvector is $\lambda_{a}$ for $M_{a}$. We choose the scaling factor of $\mathrm{C}$ to satisfy

$$
\sum_{a} \lambda_{a} c_{a}=1
$$

In this case, we can define $Q_{i}=\sum_{a} c_{a} C_{a}(i)$ that satisfy

$$
Q_{i}^{2}=Q_{i} \text {. }
$$

Thus $Q_{i}$ is a projection operator. In fact, $Q_{i}$ is given by

$$
Q_{i}=|G|^{-1} \sum_{h \in G} Q_{h}(i),
$$

where $|G|$ is the number of elements in the group $G$. We can check explicitly that

$$
\begin{aligned}
Q_{i}^{2} & =|G|^{-2} \sum_{h, h^{\prime}} Q_{h}(i) Q_{h^{\prime}}(i)=|G|^{-2} \sum_{h, h^{\prime}} Q_{h h^{\prime}}(i) \\
& =|G|^{-1} \sum_{h} Q_{h}(i)=Q_{i} .
\end{aligned}
$$

\section{A commuting-projector Hamiltonian}

We note that $Q_{h}(i)$ 's generate the local gauge transformations. Since the closed-string operators are gauge invariant, we have (for closed-string operators)

$$
\begin{aligned}
{\left[B_{q}\left(S_{\text {closed }}^{1}\right), C_{a}(i)\right] } & =0, \\
{\left[B_{q}\left(S_{\text {closed }}^{1}\right), B_{q^{\prime}}\left(S_{\text {closed }}^{1 \prime}\right)\right] } & =0, \\
{\left[C_{a}(i), C_{b}(j)\right] } & =0 .
\end{aligned}
$$

Therefore, we can construct the following commuting projector Hamiltonian $[5,74]$

$$
H=U \sum_{i}\left(1-Q_{i}\right)+J \sum_{\langle i j k l\rangle}\left(1-B_{\langle i j k l\rangle}\right),
$$

where $U, J>0$,

$$
B_{\langle i j k l\rangle}=\sum_{i} \frac{d_{q}}{D^{2}} B_{q}(\langle i j k l\rangle)
$$

and $\langle i j k l\rangle$ labels the loops around the squares of the cubic lattice.

The ground state of the above exactly solvable Hamiltonian has a nontrivial topological order. The low-energy effective theory is the $G$-gauge theory $[5,74]$.

\section{The point-like and string-like excitations}

What are the excitations for the above Hamiltonian? There are local point-like excitations created by local operators. There are also topological point-like excitations that cannot be created by local operators. Two topological point-like excitations are said to be equivalent if they differ by local point-like excitations. The equivalent topological point-like excitations are said to have the same type.

We note that the closed string operators $B_{q}\left(S_{\text {closed }}^{1}\right)$ (84) commute with the Hamiltonian (106). Thus, the string operators act within the ground-state subspace. We see that the ends of the open string operators create point-like excitations, which are labeled by representations $R_{q}$. The types of topological point-like excitations one-to-one correspond to the irreducible representations of $G$. In other words, topological point-like excitations are described by representations of $G$ in a $G$-gauge theory.

Similarly, there are also topological string-like excitations. They are created at the boundary of the open membrane operators. To define the membrane operators, we point out that a membrane $\tilde{S}^{2}$ is formed by the faces of the dual lattice, which is also a cubic lattice. The faces of the dual lattice correspond to the links in the original lattice and are also labeled by $i j$. Let us first assume $G$ is Abelian. In this case, the membrane operators are defined as

$$
C_{h}\left(\tilde{S}^{2}\right)\left|\left\{g_{i j}\right\}\right\rangle=\prod_{i j \in \tilde{S}^{2}} T_{i j}(h)\left|\left\{g_{i j}\right\}\right\rangle,
$$

where the operator $T_{i j}(h)$ acts only on link $i j$ and is defined as

$$
T_{i j}(h)\left|g_{i j}\right\rangle=\left|h g_{i j}\right\rangle \quad \text { or } \quad T_{i j}(h)\left|g_{j i}\right\rangle=\left|g_{j i} h^{-1}\right\rangle .
$$

We see that $C_{h}\left(\tilde{S}^{2}\right)$ simply shifts $g_{i j}$ on the membrane $\tilde{S}^{2}$ by $h$.

For non-Abelian $G$, the membrane operators are given by

$$
C_{a}\left(\tilde{S}^{2}\right)\left|\left\{g_{i j}\right\}\right\rangle=\sum_{h \in \chi_{a}} \prod_{i j \in \tilde{S}^{2}} T_{i j}\left(h_{i j}\right)\left|\left\{g_{i j}\right\}\right\rangle,
$$

where $\chi_{a}$ is the $a$ th conjugacy class of $G$. In the $\prod_{i j \in \tilde{S}^{2}}, i$ 's are on one side of the membrane and $j$ 's are on the other side of the membrane (see Fig. 15). Lastly, $h_{i j}$ is a function of $h$ and $g_{i j}$. For non-Abelian group $G, h_{i j}$ is complicated, but when all $g_{i j}=1, h_{i j}$ has a simple form $h_{i j}=h$. For general $g_{i j}$, we need to choose a base point $i_{0}$ on one side of the membrane, and a path $i_{0} \rightarrow i$ on the membrane that connect the base point $i_{0}$ to any other point $i$ on the membrane (see Fig. 15). Then we can define $h_{i j}$ as

$$
h_{i j}=\left(g_{i_{0} i^{\prime}} \ldots g_{i^{\prime \prime} i}\right)^{-1} h\left(g_{i_{0} i^{\prime}} \ldots g_{i^{\prime \prime}}\right),
$$




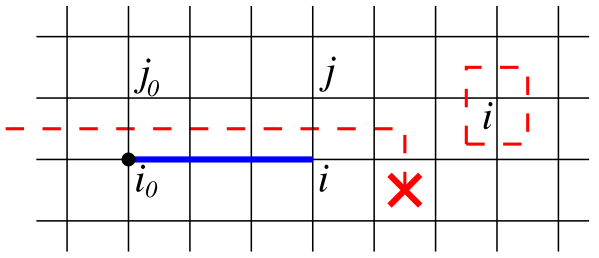

FIG. 15. The red dashed lines are membranes and the cross marks the boundary of the membranes. The blue thick line is the path $i_{0} \rightarrow i$.

where $\left(g_{i_{0} i^{\prime}} \ldots g_{i^{\prime \prime} i}\right)$ is the product of the link variables along the path $i_{0} \rightarrow i$.

We note that when the closed membrane encloses only one site $i$ (see Fig. 15), the operator $C_{a}\left(\tilde{S}^{2}\right)$ reduces to $C_{a}(i)$ as discussed before:

$$
C_{a}\left(\tilde{S}^{2}\right)=C_{a}(i)
$$

Thus, $C_{a}(i)$ can be viewed as a small membrane operator, rather than a point operator.

Let us consider a loop $i_{0} \rightarrow i \rightarrow j \rightarrow j_{0} \rightarrow i_{0}$. The $G$ flux through such a loop in the ground state $\left|\Psi_{0}\right\rangle$ is trivial: $\left(g_{i_{0} i^{\prime}} \ldots g_{i^{\prime \prime} i}\right) g_{i j}\left(g_{j j^{\prime}} \ldots g_{j^{\prime \prime} j_{0}}\right) g_{j_{0} i_{0}}=1$. This is because the ground state $\left|\Psi_{0}\right\rangle$ satisfies

$$
B\left|\Psi_{0}\right\rangle=\left|\Psi_{0}\right\rangle .
$$

After we apply the membrane operator (110), the $G$-flux through the same loop becomes

$$
\begin{aligned}
& \left(g_{i_{0} i^{\prime}} \ldots g_{i^{\prime \prime} i}\right) h_{i j} g_{i j}\left(g_{j j^{\prime}} \ldots g_{j^{\prime \prime} j_{0}}\right) g_{j_{0} i_{0}} h^{-1} \\
& \quad=h\left(g_{i_{0} i^{\prime}} \ldots g_{i^{\prime \prime} i}\right) g_{i j}\left(g_{j j^{\prime}} \ldots g_{j^{\prime \prime} j_{0}}\right) g_{j_{0} i_{0}} h^{-1}=1,
\end{aligned}
$$

which is still trivial. This allows us to conclude that for states $|\Psi\rangle$ satisfying $B|\Psi\rangle=|\Psi\rangle$ and for closed membrane $\tilde{S}_{\text {closed }}^{2}$, we have

$$
B C_{a}\left(\tilde{S}_{\text {closed }}^{2}\right)|\Psi\rangle=C_{a}\left(\tilde{S}_{\text {closed }}^{2}\right)|\Psi\rangle .
$$

We can also show that

$$
Q_{g}(i) C_{a}\left(\tilde{S}^{2}\right)=C_{a}\left(\tilde{S}^{2}\right) Q_{g}(i) .
$$

For example,

$$
\begin{aligned}
Q_{g}^{-1}\left(i_{0}\right) C_{a}\left(\tilde{S}^{2}\right) Q_{g}\left(i_{0}\right) & =\sum_{g^{-1} h g \in \chi_{a}} \prod_{i j \in \tilde{S}^{2}} T_{i j}\left(h_{i j}\right) \\
& =C_{a}\left(\tilde{S}^{2}\right) .
\end{aligned}
$$

Also,

$$
Q_{g}^{-1}(i) T_{i j}\left(h_{i j}\right) Q_{g}(i)=T_{i j}\left(\tilde{h}_{i j}\right)
$$

where

$$
\tilde{h}_{i j}=g^{-1}\left(g_{i_{0} i^{\prime}} \ldots g_{i^{\prime \prime} i} g^{-1}\right)^{-1} h\left(g_{i_{0} i^{\prime}} \ldots g_{i^{\prime \prime} i} g^{-1}\right) g=h_{i j} .
$$

Thus, in general, we have

$$
\begin{gathered}
Q_{g}^{-1}(i) C_{a}\left(\tilde{S}^{2}\right) Q_{g}(i)=C_{a}\left(\tilde{S}^{2}\right), \\
C_{b}(i) C_{a}\left(\tilde{S}^{2}\right)=C_{a}\left(\tilde{S}^{2}\right) C_{b}(i),
\end{gathered}
$$

for any $i$, even for open membranes. The results (115) and (120) imply that closed membrane operators $C_{a}\left(\tilde{S}_{\text {closed }}^{2}\right)$ act within the ground-state subspace of the Hamiltonian (106). Therefore, the boundary of the open membrane operators (110) creates string-like excitations, which are labeled by conjugacy classes $\chi_{a}$.

\section{E. Exact algebraic higher symmetry}

Since the Hamiltonian (106) commutes with the closed string operators $B_{q}\left(S_{\text {closed }}^{1}\right)$,

$$
\left[H, B_{q}\left(S_{\text {closed }}^{1}\right)\right]=0,
$$

we say that the Hamiltonian has an algebraic 2-symmetry generated by $B_{q}\left(S_{\text {closed }}^{1}\right)$ for any closed strings. Since the composition of the symmetry transformations satisfies the fusion rule (87), there is not a group multiplication rule for non-Abelian $G$. Thus, the $B_{q}$ (closed string)'s generate an exact algebraic 2-symmetry which is not a higher 2-symmetry. However, when $G$ is Abelian, $B_{q}\left(S_{\text {closed }}^{1}\right)$ 's generate a higher 2-symmetry.

There is another way to describe the algebraic 2-symmetry using the open string operators [19]. We note that the Hamiltonian is a sum of local operators $H=\sum_{i} H_{i}$, where $H_{i}$ acts only on the degrees of freedom near site $i$. We find that $H_{i}$ commutes with open string operators as long as the ends of the strings are a distance away from the site $i$ :

$$
\left[H_{i}, B_{q}\left(S_{\text {open }}^{1}\right)\right]=0 .
$$

\section{F. Emergent algebraic higher symmetry}

We also note that the Hamiltonian (106) commutes with $U_{h}\left(S^{3}\right)$

$$
\left[H, U_{h}\left(S^{3}\right)\right]=0, \quad U_{h}=\prod_{i} Q_{h}(i), \quad h \in G .
$$

Thus, the Hamiltonian has a 0-symmetry, i.e., a global symmetry described by symmetry group $G$. In fact, the Hamiltonian has a much bigger symmetry. It has a local symmetry described by group $G^{N_{v}}$, where $N_{v}$ is the number of lattice sites:

$$
\left[H, Q_{h_{i}}(i)\right]=0, \quad h_{i} \in G .
$$

On the other hand, the membrane operators $C_{a}\left(\tilde{S}_{\text {closed }}^{2}\right)$ 's do not commute with the Hamiltonian (106). Thus, the Hamiltonian does not have algebraic 1-symmetries. However, $C_{a}\left(\tilde{S}_{\text {closed }}^{2}\right)$ acts within the degenerate ground subspace. More generally, $C_{a}\left(\tilde{S}_{\text {closed }}^{2}\right)$ and $H$ commute in the subspace where $B_{\langle i j k l\rangle}=1$ (i.e., in the finite energy subspace of $H$ when $J \rightarrow+\infty$ ). Therefore, the Hamiltonian has an emergent low-energy algebraic 1-symmetry generated by $C_{a}\left(\tilde{S}_{\text {closed }}^{2}\right)$ 's when $J \rightarrow+\infty$. Such an emergent algebraic 1-symmetry is a (group-like) 1-symmetry only when $G$ is Abelian.

\section{DESCRIPTION OF ALGEBRAIC HIGHER SYMMETRY IN A SYMMETRIC PRODUCT STATE}

Usually, we use the symmetry transformation, i.e., the symmetry group $G$, to describe a symmetry. We can also use 
the symmetry charges, i.e., the representations $\mathcal{R e p}(G)$, to describe a symmetry. Due to Tannaka duality, the two descriptions are equivalent. In last section, we introduced algebraic higher symmetry via the symmetry transformations. In this and next sections, we will develop a similar dual way to describe algebraic higher symmetry, i.e., via the representations of algebraic higher symmetry. This section will concentrate on the point-of-view-based lattice model and symmetric product state. The next section will present a point of view based on a higher category.

What are the representations of algebraic higher symmetry? Physically, the representations correspond to the "charged excitations" in a symmetric ground state which has a trivial topological order (i.e., be a product state). In the following, we will explore the following questions: What is a "symmetric state" (i.e., no spontaneous symmetry breaking) for algebraic higher symmetry? What kind of algebraic higher symmetry can have symmetric ground state with no topological order? What are the "charged excitations" for algebraic higher symmetry? This allows us to obtain a representation theory for algebraic higher symmetry, in terms of local fusion higher category.

\section{A. Spontaneous broken and unbroken algebraic higher symmetry}

In Sec. IV, we constructed a $3 d$ lattice model that has an exact algebraic 2-symmetry generated by string operators $B_{q}\left(S^{1}\right)$. However, the ground state of the model (106) spontaneously breaks the algebraic 2-symmetry, which gives us a topological order described by the $G$-gauge theory.

Here, we consider a different model,

$$
H=-V \sum_{i j} \delta\left(g_{i j}\right)+U \sum_{i}\left(1-Q_{i}\right)+J \sum_{\langle i j k l\rangle}\left(1-B_{\langle i j k l\rangle}\right)
$$

by including an extra term $-V \delta\left(g_{i j}\right)$ and taking $J \rightarrow+\infty$ limit. Here,

$$
\delta(g)=\left\{\begin{array}{ll}
1, & \text { if } g=\mathrm{id} \\
0, & \text { otherwise }
\end{array} .\right.
$$

The model also has the algebraic 2-symmetry $\left[H, B_{q}\left(S_{\text {closed }}^{1}\right)\right]=0$. If we choose the limit $U \ll V$, the ground state is given by $\left|\left\{g_{i j}=1\right\}\right\rangle$. This ground state does not spontaneously break the algebraic 2-symmetry.

For the usual global symmetry, the spontaneous symmetry breaking is defined via nonzero order parameters. Here we would like to define the spontaneous symmetry breaking of algebraic higher symmetry in a different way:

Definition $^{\text {ph }} 24$. An algebraic higher symmetry is spontaneously broken if there exists a close space, such that the symmetry transformations are not proportional to the identity operator in the nearly degenerate ground-state subspace on that space.

For the Hamiltonian (125), the ground state is not degenerate on any closed spaces. Thus, the algebraic 2-symmetry is not spontaneously broken. In contrast, for model (106), the ground states are degenerate on space $S_{x}^{1} \times S_{y}^{1} \times S_{z}^{1}$.
The different ground states can have different fluxes, say, $\prod_{(i j) \in S_{x}^{1}} g_{i j}=h$. The symmetry generator $B_{q}\left(S_{x}^{1}\right)$ is not proportional to identity, since $B_{q}\left(S_{x}^{1}\right)=X_{q}(h)$ on the ground state with flux $h$. Here,

$$
X_{q}(g)=\operatorname{Tr}\left[R_{q}(g)\right], \quad g \in G
$$

is the character of the representation $R_{q}$. We see that the ground state of the model (106) spontaneously breaks the algebraic 2-symmetry $B_{q}\left(S^{1}\right)$. In fact, the algebraic 2-symmetry is completely broken, which gives rise to the topological order described by the $G$-gauge theory.

\section{B. Anomaly-free algebraic higher symmetry}

In this section, we would like to discuss algebraic higher symmetry in the simplest state-symmetry unbroken state without topological order. However, some algebraic higher symmetries may not allow such a state. This leads to an important attribute of algebraic higher symmetry. There are two ways to describe this attribute: The first is a microscopic way:

Definition $^{p h} 25$. An algebraic higher symmetry in a lattice system is anomaly-free if a system with the symmetry allows a phase which has a symmetric product state as its unique gapped ground state.

The second is a macroscopic way:

Definition $^{p h}$ 26. An algebraic higher symmetry is anomaly-free if a system with the symmetry allows a phase which has a unique gapped ground state on each closed space. Such a phase is also symmetric.

For 0-symmetry on lattice, we can use on-siteness to define anomaly-free 0-symmetry [3]. Using this definition, we believe that all anomalous (non-on-site) 0 -symmetry can be realized on a boundary of a system in one higher dimension with anomaly-free (on-site) 0-symmetry [3]. For finite symmetries, we believe that there is an one-to-one correspondence between anomalous 0-symmetries and the SPT order in one higher dimension [3]. (While for infinite symmetry described by a continuous compact group, we do not have the one-to-one correspondence between anomalous 0-symmetries and the SPT order in one higher dimension [3].) As a result, the finite anomalous 0 -symmetries are classified by the SPT orders in one higher dimension. Since we believe that the boundary uniquely determines the bulk [25,27], the above result also implies that an anomalous 0 -symmetry does not allow a gapped symmetric product state as the ground state [86,87]. Otherwise, the SPT order in one higher dimension must be trivial, as implied by such a symmetric ground state on the boundary.

For algebraic higher symmetry, it is hard to define onsiteness. So we turn things around and use the existence of trivial symmetric gapped ground state to define algebraic higher symmetry (where trivial means product state). In this case, algebraic higher symmetry can appear at a boundary of the trivial SPT phase for algebraic higher symmetry. The boundary of nontrivial SPT phases for an algebraic higher symmetry realize an anomalous algebraic higher symmetry. In this paper, we only consider anomaly-free algebraic higher symmetries. 


\section{The charge objects and charge creation operators for the exact algebraic 2-symmetry}

The exact algebraic 2-symmetry in the lattice model (125) is generated by $B_{q}\left(S_{\text {closed }}^{1}\right)$. The algebraic 2 -symmetry is anomaly-free since the model (125) allows symmetric gapped product state $\left|\left\{g_{i j}=1\right\}\right\rangle$ as its unique ground state.

The charge objects of such a 2-symmetry live on 2dimensional surfaces [just like the charges of a 0 -symmetry (the usual global symmetry) live on 0-dimensional points]. To construct the 2-dimensional operators that create the charge objects of the algebraic 2-symmetry, let us review the charge creation operators for the 0 -symmetry in a proper general setting.

A pair of charge and anticharge of a 0 -symmetry is created by an operator $C\left(S^{0}\right)$ on $S^{0}$ (i.e., on two points $i$ and $j$ ), for example,

$$
C\left(S^{0}\right)=\sum_{a} \psi_{a}^{\dagger}(i) \psi_{a}(j),
$$

where the local operator $\psi_{a}(i)$ forms a unitary representation $R_{a b}$ for the 0 -symmetry group $G$ :

$$
U_{g} \psi_{a}=\sum_{b} R_{a b}(g) \psi_{b} U_{g}, \quad g \in G .
$$

We note that when the two points in $S^{0}$ belong to the same connected component of the space, $C\left(S^{0}\right)$ commutes with the algebraic 0 -symmetry transformations and creates an neutral excitation. On part of $S^{0}$, the creation operator becomes $\psi_{a}(i)$, which creates a non-neutral excitation.

Similarly, a neutral charge object of a $k$-symmetry is created by operators on closed contractible $k$-dimensional manifolds, such as $S^{k}$. Such an operator on contractible $S^{k}$ commutes with the algebraic $k$-symmetry transformations and creates an neutral excitation. A charge object of $k$-symmetry is created by operators $C\left(M^{k}\right)$ on open $k$-dimensional manifold $M^{k}$. In $n$ d, when the algebraic $k$-symmetry generator $B_{q}\left(S_{\text {closed }}^{n-k}\right)$ on $(n-k)$-dimensional sub-manifold intersects with the submanifold $M^{k}$ at one point, we can detect the $k$ symmetry charge. The algebra between symmetry generators $B_{q}\left(S_{\text {closed }}^{n-k}\right)$ and charge creation operators $C\left(M^{k}\right)$ only depends on the linking between $S_{\text {closed }}^{n-k}$ and $\partial M^{k}$, and does not depend on the deformations of $S_{\text {closed }}^{n-k}$ and $\partial M^{k}$ that do not change their linking. Those are key conditions for the charge creation operators $C\left(M^{k}\right)$ for an algebraic higher symmetry.

For our algebraic 2-symmetry in $3 \mathrm{~d}$, the charge creation operator acts on 2-dimensional surfaces with or without boundary. In fact, such a charge creation operator is nothing but the membrane operator $C_{a}\left(\tilde{S}^{2}\right)$ discussed in Sec. IV D. The charge object created by $C_{a}\left(\tilde{S}^{2}\right)$ can be detected by the 2-symmetry generator $B_{q}\left(S_{\text {closed }}^{1}\right)$, when $\tilde{S}^{2}$ has a boundary, or when $\tilde{S}^{2}$ is closed and noncontractible.

In fact, on the $\left|\left\{g_{i j}=1\right\}\right\rangle$ ground state, the creation operator can have a simpler form

$$
\tilde{C}_{h}\left(\tilde{S}^{2}\right)=\prod_{i j \in \tilde{S}^{2}} T_{i j}(h),
$$

where $\prod_{i j \in \tilde{S}^{2}}$ is over all the links $i j$ of the original lattice that form the faces in $\tilde{S}^{2}$ of the dual lattice. Such an operator

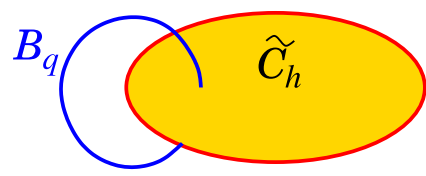

FIG. 16. In 3-dimensional space, a disk-like 2-charge object (a 2-dimensional excitation) created by $\tilde{C}_{h}\left(\tilde{S}^{2}\right)$ can be detected by the algebraic 2-symmetry transformation loop operator $B_{q}\left(S_{\text {closed }}^{1}\right)$.

just changes $g_{i j}=1$ to $g_{i j}=h$ on links $i j$ of the original lattice that form the faces in $\tilde{S}^{2}$ of the dual lattice. $g_{i j}=h$ on $\tilde{S}^{2}$ corresponds to a charged excitation, called a 2 -charge object labeled by $h$, of our algebraic 2 -symmetry generated by $B_{q}\left(S_{\text {closed }}^{1}\right)$.

If $\tilde{S}^{2}$ is a disk in 3d space, then the 2-charge object created by $\tilde{C}_{h}\left(\tilde{D}^{2}\right)$ can be detected by the algebraic 2 -symmetry operator $B_{q}\left(S_{\text {closed }}^{1}\right)$ if $S_{\text {closed }}^{1}$ is linked with $\partial \tilde{S}^{2}$-the boundary of the 2-charge object (see Fig. 16). If fact, $B_{q}\left(S_{\text {closed }}^{1}\right)=$ $\operatorname{Tr} R_{q}(h)$ in this case when acting on the 2-charge object. In comparison, for the ground state $\left|\left\{g_{i j}=1\right\}\right\rangle$, the 2-symmetry generator is equal to the dimension $d_{q}$ of the $q$-representation: $B_{q}\left(S_{\text {closed }}^{1}\right)=\operatorname{Tr} R_{q}(1)=d_{q}$. We see that the algebraic 2symmetry cannot distinguish two 2-charge objects labeled by $h$ and $h^{\prime}$ if $h$ and $h^{\prime}$ belong to the same conjugacy class, so the distinct algebraic 2 -symmetry charges are labeled by the conjugacy classes $\chi_{a}$ of $G$.

We stress that the membrane operator $C_{a}\left(\tilde{S}^{2}\right)$ that creates the 2-dimensional charge object of the algebraic 2-symmetry is an operator that acts only on the membrane $\tilde{S}^{2}$. This is a very important general feature.

Proposition 23. On top of a ground state that does not break the symmetry, the $k$-dimensional charge object of an algebraic $k$-symmetry is created by an operator that acts only on the $k$-dimensional subspace that supports the charge object.

We note that in $J \rightarrow \infty$ limit, only 2 -charge objects corresponding to closed surfaces have low energy. The 2-charge objects corresponding to surfaces with boundary cost energy of order $J$ or bigger. We may consider the low-energy subspace of the model in $J \rightarrow \infty$ limit. In fact, we consider an even smaller space, the invariant sub-Hilbert space of all the 2symmetry transformations generated by $B_{q}\left(S_{\text {closed }}^{1}\right)$ operators. The collection of those created 2-charge objects within the symmetric sub-Hilbert space, plus their fusion (and braiding) properties, form a higher category. The 2-charge objects are labeled by $h \in G$ and created by $\tilde{C}_{h}\left(\tilde{S}_{\text {closed }}^{2}\right)$. The fusion of $\tilde{C}_{h}\left(\tilde{S}_{\text {closed }}^{2}\right)$ is given by

$$
\tilde{C}_{h}\left(\tilde{S}_{\text {closed }}^{2}\right) \otimes \tilde{C}_{h^{\prime}}\left(\tilde{S}_{\text {closed }}^{2}\right)=\tilde{C}_{h h^{\prime}}\left(\tilde{S}_{\text {closed }}^{2}\right) .
$$

The charged membrane-like excitations, labeled by $h \in G$, form a fusion 3-category $\mathcal{R}=3 \mathcal{V e c}_{G}$ (see Definition 17), which is also a local fusion 3-category (see Definition 27). We also refer $\mathcal{R}=3 \mathcal{V e c}_{G}$ as the representation category of the algebraic 2 -symmetry. Physically, $\mathcal{R}$ is the fusion 3-category that describes the low-energy excitations in model (125).

What is a fusion higher category and what is a local fusion higher category? Roughly speaking, a fusion higher category describes the point-like, string-like, etc., excitations above a gapped liquid ground state. If an excitation can be annihilated by an operator acting on the excitations, then we say the 
TABLE II. Correspondence between concepts in fusion higher category and concepts in topological order $[25,26]$.

\begin{tabular}{ll}
\hline \hline Concepts in higher category & \multicolumn{1}{c}{ Concepts in physics } \\
\hline Fusion $n$-category $\mathcal{C}$ & $\begin{array}{c}\text { A collection of all the types of codimension-1 and higher excitations (plus their fusion and braiding } \\
\text { properties) in an } n \text { d (potentially anomalous) topological order } \\
\text { The types of codimension-1 topological excitations. They can fuse }\end{array}$ \\
Simple objects of $\mathcal{C}$ & The types of codimension-2 topological excitations. They can fuse and braid. \\
Simple 1-morphisms of $\mathcal{C}$ & The types of string-like topological excitations \\
Simple $(n-2)$-morphisms of $\mathcal{C}$ & The types of point-like topological excitations \\
Simple $(n-1)$-morphisms of $\mathcal{C}$ & The local operators acting on the point-like excitations \\
$n$-morphisms of $\mathcal{C}$ & The "charged" excitations (charge objects) above a product state of a bosonic system with an algebraic \\
Local fusion $n$-category $\mathcal{R}$ & higher symmetry $\mathcal{R}$. It is called the representation category of the algebraic higher symmetry
\end{tabular}

excitation is local. Note that the operators may break any symmetry and may not be local, as long as they act on the support subspace of the excitation. The fusion higher category formed by local excitations is a local fusion higher category. Since the membrane excitations in $\mathcal{R}$ can all be annihilated by operators on the membranes, $\mathcal{R}$ is a local fusion higher category.

The following discussions use the notions of topological order higher categories extensively [25,26,29,30], which are reviewed in Sec. III. Table II summarizes some related concepts in higher category and in topological order.

\section{LOCAL FUSION HIGHER CATEGORY AND REPRESENTATIONS (CHARGE OBJECTS) OF ANOMALY-FREE ALGEBRAIC HIGHER SYMMETRY}

In the last section, we described the charged excitations (i.e., the charge objects) in a trivial symmetric ground state with anomaly-free algebraic higher symmetry. Here trivial state means a product state. In the rest of this paper, we will mainly discuss anomaly-free algebraic higher symmetry, and we drop "anomaly-free" for simplicity.

For a 0 -symmetry $G$, we know that its charges are representations of $G$. All those representations form a symmetric fusion category $\operatorname{Rep} G$. Because of the Tannaka duality, we can use the local fusion category $\operatorname{Rep} G$ to fully describe the symmetry group $G[45,46]$. To be more precise, the charges (the representations) of $G$ correspond to point-like excitations. Those point-like charges can condense to form other descendent excitations. All those excitations are described by a fusion $n$-category, if the 0 -symmetry $G$ lives in $n$-dimensional space. We denote such a fusion $n$-category as $n \mathcal{R}$ ep $G$. In other words, an $n$ d 0 -symmetry $G$ is fully characterized by a symmetric fusion $n$-category $n \mathcal{R} \operatorname{ep} G$. We refer to $n \mathcal{R} \operatorname{ep} G$ as the representation category of the 0 -symmetry $G$.

In the above, we try to use excitations (trapped by the symmetric traps) to characterize a symmetry. Here we would like to stress that the excitations described by the fusion $n$-category $n \mathcal{R}$ ep $G$ only correspond to the excitations in the symmetric sub-Hilbert space $\mathcal{V}_{\text {symm }}$ of the many-body system. The fusion $n$-category $n \mathcal{R} \operatorname{ep} G$ do not include the excitations outside the symmetric sub-Hilbert space. In the thermodynamic limit, restricting to symmetric sub-Hilbert space does not affect our ability to understand the properties of a symmetric system. We would like to use a similar approach to characterize an algebraic higher symmetry (which is not characterized by groups or even higher groups): The representations (i.e., the charge objects) of an algebraic higher symmetry are simply the excitations above a symmetric product state, which are also described by a category-the local fusion higher category.

\section{A. The excitations in a symmetric state with no topological order}

To have a general understanding of the charge objects, let us consider a local lattice Hamiltonian $H$ with an algebraic higher symmetry. We assume the ground state $\left|\Psi_{\text {grnd }}\right\rangle$ of $H$ has no topological order nor SPT order, i.e., it can be deformed into a product state without a phase transition, via a symmetry-preserving path. Then how to understand the pointlike, string-like excitations, etc., of the above ground state? Also similar to the 0 -symmetry case, here we only consider the symmetric excitations (i.e., those trapped by symmetric traps) in the symmetric sub-Hilbert space $\mathcal{V}_{\text {symm. }}$. We know that an algebraic higher symmetry is generated by many operators acting on all closed submanifolds. The symmetric sub-Hilbert space is the invariant sub-Hilbert space of all those symmetry generators.

To understand the excitations, first let us define excitations more carefully. For example, to define string-like excitations, we can add several trap Hamiltonians $\Delta H_{\text {str }}\left(S_{i}^{1}\right)$ to $H$ such that $H+\sum_{i} \Delta H_{\text {str }}\left(S_{i}^{1}\right)$ has an energy gap. $\Delta H_{\text {str }}\left(S_{i}^{1}\right)$ is only nonzero along the string $S_{i}^{1}$ and commutes with the generators of the algebraic higher symmetry. We also assume $\Delta H_{\mathrm{str}}\left(S_{i}^{1}\right)$ to be stable: Any small symmetric change of $\Delta H_{\mathrm{str}}\left(S_{i}^{1}\right)$ does not change the ground-state degeneracy in the large string $S_{i}^{1}$ limit. The resulting string corresponds to a simple morphism in mathematics. We also define two strings labeled by $\Delta H_{\text {str }}\left(S^{1}\right)$ and $\Delta \tilde{H}_{\text {str }}\left(\tilde{S}^{1}\right)$ as equivalent, if we can deform $\Delta H_{\text {str }}\left(S^{1}\right)$ into $\Delta \tilde{H}_{\text {str }}\left(\tilde{S}^{1}\right)$ without closing the energy gap while preserving the algebraic higher symmetry. The equivalent classes of the strings define the types of the strings (see Definition 10).

In the example in Sec. V, the 2-dimensional charge object of an algebraic 2-symmetry is created by a membrane operator. If the membrane is a closed 2-dimensional subspace, then the membrane operator acts within the symmetric sub-Hilbert space $\mathcal{V}_{\text {symm }}$ and creates an excitation in the fusion higher category. If the membrane has a boundary, then the membrane 
operator does not act within the symmetric sub-Hilbert space and creates an excitation not in the fusion higher category. When the membrane has a boundary, such a boundary is the morphism that connect the membrane excitation to the trivial excitation. In the above example, such a boundary (i.e., the morphism) is not allowed, since it breaks the algebraic 2-symmetry (i.e., the membrane with the boundary does not act within the symmetric sub-Hilbert space).

\section{B. Local fusion higher category}

Now we are ready to define a local fusion higher category, which describes the collection of excitations (i.e., the collection of types) in the system mentioned above, i.e., a system with algebraic higher symmetry whose ground state is a symmetric bosonic product state without degeneracy. Also, we only consider excitations within the symmetric sub-Hilbert space $\mathcal{V}_{\text {symm. }}$. For a symmetric trivial phase without topological order, it has only local excitations. From a categorical point of view, a local excitation can always be connected to the trivial excitation through a morphism as described above, if we are willing to break the symmetry. However, if we preserve the symmetry, the symmetry-breaking morphism is not allowed and some excitations cannot connect to trivial excitation via symmetry-preserving morphisms (i.e., symmetry-preserving domain walls). This leads to the following definition: A fusion $n$-category $\mathcal{R}$ is local if we can add morphisms in a consistent way, such that all the resulting simple morphisms are isomorphic to the trivial one. Physically, this process of "adding morphisms" corresponds to explicit breaking of algebraic higher symmetry. This is because $\mathcal{R}$ only has morphisms that correspond to symmetric operators. Adding morphisms means including morphisms that correspond to symmetry-breaking operators. If after breaking all the symmetry, $\mathcal{R}$ describes a trivial phase without symmetry, then $\mathcal{R}$ is a local fusion $n$-category. The above can be stated more precisely as follows:

Definition 27. A fusion $n$-category $\mathcal{R}$ (see Definition 17 ) equipped with a top-faithful surjective monoidal functor $\beta$ from $\mathcal{R}$ to the trivial fusion $n$-category: $\mathcal{R} \stackrel{\beta}{\rightarrow} n \mathcal{V}$ ec is called a local fusion $n$-category. Here, top-faithful means that the functor $\beta$ is injective when acting on the top morphisms (i.e., the $n$-morphism in this case).

Remark 5. The top-faithful condition means that operators in $\mathcal{R}$ form a subset of operators in $n \mathcal{V}$ ec, which agrees with the physical interpretation that from $\mathcal{R}$ to $n \mathcal{V}$ ec we add symmetrybreaking operators. The functor $\beta$ may not be faithful when acting on other morphisms. In other words, every object and morphism in $\mathcal{R}$ can be viewed as (i.e., can be mapped into) objects and morphisms in $n \mathcal{V}$ ec, but the map may not be injective.

When we are interested in fermion systems, we need to replace $n \mathcal{V}$ ec for $n \mathrm{~s} \mathcal{V e c}$. More generally, the building blocks of our physical system may have even larger intrinsic symmetry (which is unbreakable or we are not willing to break) besides the fermion number parity. Let $\mathcal{V}$ denote the fusion $n$-category formed by the building blocks $(\mathcal{V}=n \mathcal{V e c}$ for bosons, $\mathcal{V}=$ $n s \mathcal{V}$ ec for fermions, and possibly any other $\mathcal{V}$ for more exotic cases such as an effective theory built upon anyons). We define the notion of $\mathcal{V}$-local fusion $n$-categories.
Definition 28. A $\mathcal{V}$-local fusion $n$-category is a fusion $n$ category $\mathcal{R}$ equipped with a top-faithful surjective monoidal functor $\beta: \mathcal{R} \rightarrow \mathcal{V}$.

\section{Local fusion 1-category $\mathcal{R e p} G$ and $\mathcal{V e c}_{G}$}

As an example, let us consider a 1d system with degrees of freedom labeled by $g_{i} \in G$ on site $i$, where $G$ is a group. The Hamiltonian of the system is given by

$$
H=-J \sum_{i} \sum_{h \in G} T_{i}(h)-V \sum_{i} \delta\left(g_{i-1}^{-1} g_{i}\right),
$$

where $T_{i}(h)$ is an operator

$$
\begin{aligned}
& T_{i}(h)\left|\ldots, g_{i-1}, g_{i}, g_{i+1}, \ldots\right\rangle \\
& \quad=\left|\ldots, g_{i-1}, h g_{i}, g_{i+1}, \ldots\right\rangle, \quad h \in G .
\end{aligned}
$$

The system has a symmetry $G$

$$
\left|\ldots, g_{i-1}, g_{i}, g_{i+1}, \ldots\right\rangle \rightarrow\left|\ldots, g g_{i-1}, g g_{i}, g g_{i+1}, \ldots\right\rangle \text {. }
$$

When $J \gg|V|$, the ground state is a product state $\left|\Psi_{\text {grnd }}\right\rangle=$ $\otimes_{i}|0\rangle_{i}$, where $|0\rangle_{i} \equiv|G|^{-1 / 2} \sum_{g}|g\rangle_{i}$, that does not spontaneously break the symmetry.

Note that $\left\{|g\rangle_{i}, g \in G\right\}$ spans the regular representation of $G$. It can be further decomposed into irreducible representations. Let $|a\rangle_{i},|b\rangle_{i}, \ldots$ be a basis in an irreducible representation. Under the symmetry transformation $h \in G$, $|a\rangle_{i}$ transforms to $T_{i}(h)|a\rangle_{i}=\sum_{b} R_{a b}(h)|b\rangle_{i}$, where $R_{a b}(h)$ is the matrix representing $h$. A pointlike excitation at site $i$ is created by changing the state $|0\rangle_{i}$ on site $i$ to $|a\rangle_{i}=\sum_{g}\langle g \mid a\rangle|g\rangle_{i}$. Since

$$
\begin{aligned}
T_{i}(h)|a\rangle_{i} & =\sum_{g}\langle g \mid a\rangle|h g\rangle_{i}=\sum_{g}\left\langle h^{-1} g \mid a\right\rangle|g\rangle_{i} \\
& =\sum_{b} R_{a b}(h)|b\rangle_{i},
\end{aligned}
$$

we see $\left\langle h^{-1} g \mid a\right\rangle=\sum_{b} R_{a b}(h)\langle g \mid b\rangle$.

Such a ground state plus its excitations are described by a fusion 1-category $\mathcal{R e p} G$ whose objects correspond to the point-like excitations (i.e., the representations $R$ of $G$ ). The 1-morphisms of $\mathcal{R e p} G$ correspond to the symmetric local operators that act on each site. We see that the 1-morphisms directly act on the point-like excitations (the objects). If we view an excitation (an object) as a world line in spacetime, an 1-morphism that changes the excitation can be viewed as a "domain wall" on the world line. For a symmetric system, all those 1-morphisms should be symmetric operators. Respecting to those symmetric 1-morphisms, the excitations corresponding to the irreducible representations are simple objects. Different irreducible representations cannot be connected by symmetric operators, i.e., different simple objects cannot be connected by 1 -morphisms.

If we add the additional 1-morphisms that correspond to local operators that break all the symmetry, then the excitations corresponding to the irreducible representations $R$ are still allowed, but they are no longer simple objects, and split 
into direct sum of trivial excitations:

$$
R \rightarrow \underbrace{\mathbb{C} \oplus \cdots \oplus \mathbb{C} .}_{\operatorname{dim} R \text { copies }}
$$

As a result, the fusion 1-category is reduced to the trivial 1category - the category of vector spaces $\mathcal{V}$ ec. Thus, the fusion 1 -category $\operatorname{Rep} G$ is a local fusion 1-category. Indeed, all the point-like excitations can be annihilated by local operators that may break the symmetry.

Now consider a 1d system with symmetry $G$, whose ground state spontaneously breaks all the symmetry. In this case, the ground states are $|G|$-fold degenerate and are labeled by the group elements: $\left|\Psi_{g}\right\rangle, g \in G$. The point-like excitations are domain walls, which live on the links and are labeled by the elements $h$ of the group: $|h\rangle_{i_{0}, i_{0}+1}=\left|\Psi_{g, i \leqslant i_{0}} \Psi_{h g, i \geqslant i_{0}+1}\right\rangle$. Such symmetry-breaking state plus its excitations are described by a fusion 1-category $\mathcal{V e c}_{G}$, whose objects correspond to the point-like excitations (the domain walls) discussed above. We may still choose the 1-morphisms of $\mathcal{V e c}_{G}$ to be the symmetric local operators acting on the sites. However, such a choice is not proper, since such 1-morphisms cannot be viewed as the "domain walls" on the world-lines of the pointlike excitations (the domain walls on the links). In any case, let us proceed. If we add the 1-morphisms that correspond to local operators that break all the symmetry, then objects (the point-like domain-wall excitations) are confined (i.e., no longer allowed), since the ground-state degeneracy is lifted. This appears to suggest that the fusion 1-category $\mathcal{V e c}_{G}$ is not a local fusion 1-category, if we view it as describing domain walls in a spontaneous symmetry-breaking state that breaks a 0 -symmetry of group $G$. Since our choices of the 1-morphisms is not proper, the above conclusion is incorrect.

In fact, $\mathcal{V e c}_{G}$ can also be viewed as a fusion 1-category that describes excitations on top of a product state with an algebraic 0 -symmetry. The degrees of freedom on each site $i$ of our $1 \mathrm{~d}$ model are labeled by group elements of a finite group $G$. A basis of the many-body Hilbert space is given by $\left|\left\{g_{i}\right\}\right\rangle, g_{i} \in G$. The Hamiltonian is given by

$$
H=-V \sum_{i} \delta\left(g_{i}\right)-t \sum_{i, h \in G} T_{i-1, i}(h)
$$

where $T_{i-1, i}(h)$ is an operator

$$
\begin{aligned}
& T_{i-1, i}(h)\left|\ldots, g_{i-1}, g_{i}, g_{i+1}, \ldots\right\rangle \\
& \quad=\left|\ldots, g_{i-1} h^{-1}, h g_{i}, g_{i+1}, \ldots\right\rangle, \quad h \in G .
\end{aligned}
$$

The model has an algebraic 0 -symmetry generated by

$$
B_{q}=\operatorname{Tr}\left[\prod_{i} R_{q}\left(g_{i}\right)\right],
$$

where $q$ labels the representations of $G$. In the $t \rightarrow 0$ limit, the ground state is a symmetric product state $\left|\left\{g_{i}=\mathrm{id}\right\}\right\rangle$.

Above such a ground state, a point-like excitation is generated by changing $g_{i}=$ id to $g_{i}=h$ on site $i$. Thus, the excitations are labeled by group elements $h \in G$, with $h=\mathrm{id}$ corresponding to the ground state. They fuse as $h \otimes h^{\prime}=h h^{\prime}$. When the algebraic 0 -symmetry operators act on the excitations $h$, we get $B_{q}(h)=X_{q}(h)$, where $X_{q}$ is the character for the representation $q$. Those point-like excitations form a local 1 -fusion category $\mathcal{V e c}_{G}$.

The operators that break the algebraic 0 -symmetry are given by

$$
\begin{aligned}
\delta H & =T_{i}(h)\left|\ldots, g_{i-1}, g_{i}, g_{i+1}, \ldots\right\rangle \\
& =\left|\ldots, g_{i-1}, h g_{i}, g_{i+1}, \ldots\right\rangle, \quad h \in G .
\end{aligned}
$$

Those operators reduce the local 1 -fusion category $\mathcal{V e c}_{G}$ to the trivial 1 -fusion category $\mathcal{V e c}$, since those operators correspond to new morphisms $h \rightarrow h^{\prime}$ for any $h, h^{\prime} \in G$. Therefore, the 1-fusion category $\mathcal{V e c}_{G}$ is local.

We would like to mention that the $3 \mathrm{~d}$ generalization of the 1d model (137) was discussed in Sec. V. Using a similar reason, we show that the 3 -fusion category $3 \mathcal{V e c}_{G}$ is local.

\section{Representation category of algebraic higher symmetry}

Let us summarize the relation between the charge objects of an algebraic higher symmetry and a local fusion higher category.

Proposition 24. Consider an $n$ d trivial ground state which is a product state with an algebraic higher symmetry. The different types of the excitations above the ground state and within the symmetric sub-Hilbert space form a local fusion $n$-category $\mathcal{R}$ (i.e., with a fiber functor $\beta: \mathcal{R} \rightarrow n \mathcal{V e c}$ ), which is called the representation category of the algebraic higher symmetry in $n$-dimensional space.

We would like to conjecture that the Tannaka duality can be generalized to algebraic higher symmetries:

Proposition 25. There is an one-to-one correspondence between local fusion $n$-categories $\mathcal{R}$ and algebraic higher symmetries for bosonic systems in $n$-dimensional space.

In other words, the algebraic higher symmetries in $n \mathrm{~d}$ bosonic systems are fully characterized and classified by local fusion $n$-categories. Since a local fusion $n$-category $\mathcal{R}$ fully characterizes an anomaly-free algebraic higher symmetry, in this paper, an algebraic higher symmetry is denoted by $\mathcal{R}$.

We would like to remark that there are anomalous algebraic higher symmetries. For those symmetries, we cannot have trivial symmetric ground state, and it is difficult to define its representation category, since representation category, by definition, is formed by the charged excitations above the symmetric product state.

\section{E. Categorical symmetry-a holographic view of symmetry}

To gain an even deeper understanding of algebraic higher symmetry, following Ref. [19], we would like to introduce the notion of a categorical symmetry, which is a holographic point of view of a symmetry. We know that a symmetry is simply a restriction on the local operators whose sum gives rise to the Hamiltonian. Usually, the restriction is imposed by symmetry transformations, but in the holographic point of view, we do not impose restrictions via symmetric transformations. Instead, we use a topological order without any symmetry in one higher dimension to encode a symmetry. In other words, we use long-range entanglement [32] to encode a symmetry. Then the restrictions to local operators are realized via the boundary of the topological order. 
Let us consider an $n$ d system with an algebraic higher symmetry $\mathcal{R}$. When we restrict the system to the symmetric sub-Hilbert space $\mathcal{V}_{\text {symm }}$ of the algebraic higher symmetry, the system has a potentially noninvertible gravitational anomaly [24], since $\mathcal{V}_{\text {symm }}$ does not have a tensor product decomposition $\mathcal{V}_{\text {symm }} \neq \otimes_{i} \mathcal{V}_{i}$. This relates the symmetry to entanglement. Thus, the system (when restricted to the symmetric sub-Hilbert space $\mathcal{V}_{\text {symm }}$ ) can be viewed as a boundary of an anomaly-free topological order $\mathrm{M}$ in one higher dimension. The topological order $\mathrm{M}$ is described by an object in $\mathbb{T O}_{\mathrm{af}}^{n+2}$.

Which topological order $\mathrm{M}$ in one higher dimension gives rise to the desired algebraic higher symmetry $\mathcal{R}$ ? We note that $\mathcal{R}$ is a fusion $n$-category. We believe that for every fusion $n$ category $\mathcal{R}$, there is exist a unique anomaly-free topological order $\mathrm{M}$ in one higher dimension such that $\mathrm{M}$ has a boundary whose excitations realize the fusion $n$-category $\mathcal{R}$ [see (43)]. Therefore, we can find $\mathrm{M}$ from $\mathcal{R}$ via

$$
\mathrm{M}=\operatorname{bulk}(\mathcal{R})
$$

As we have discussed in Sec. II D, an $n$ d algebraic higher symmetry $\mathcal{R}$ selects a set of local operators $\left\{O_{\mathcal{R}}\right\} .\left\{O_{\mathcal{R}}\right\}$ can be viewed as a set of lattice local operators that commute with the symmetry generators, or as a set of local operators that describe all possible short-range interaction between excitations, as well as local operators that create particle-antiparticle, small loop excitations, etc., described by $\mathcal{R}$. In Sec. II F, we mentioned that an $n$ d categorical symmetry $\mathrm{M}$ also selects a local operators $\left\{O_{\mathrm{M}}\right\}$, on the boundary of $(n+1) \mathrm{d}$ topological order $\mathrm{M}$. If $\mathrm{M}=\operatorname{bulk}(\mathcal{R})$, the two sets $\left\{O_{\mathcal{R}}\right\}$ and $\left\{O_{\mathrm{M}}\right\}$ have an one-to-one correspondence and the corresponding operators has identical properties (such as identical algebraic relations for the corresponding operators). In other words, the algebraic higher symmetry $\mathcal{R}$ and the categorical symmetry $\mathrm{M}$ are holoequivalent (see Proposition 1).

Let us examine the algebraic higher symmetry $\mathcal{R}$ and the categorical symmetry $M$ in terms of their excitations. Roughly speaking, the conservation law from the symmetry is encoded in the fusion rule for the excitations. Thus, the fusion rule of the excitations with codimension-2 and higher in $\mathrm{M}$ encode the categorical symmetry M. (A codimension-1 excitation in $\mathrm{M}$ has codimension- 0 on the boundary and cannot be viewed as an excitation there.) Those excitations are described by the braided fusion $n$-category (see Sec. III H)

$$
\mathcal{M}=\Omega \mathcal{M}=\Omega^{2} \mathrm{M},
$$

where $\mathcal{M}=\Omega \mathrm{M}$ is the fusion $n$-category describing the bulk excitations in $\mathrm{M}$. As we move a bulk excitation in $\mathcal{M}$ to the boundary, it may become some boundary excitations in $\mathcal{R}$, or it may condense (i.e., becomes the trivial excitation in $\mathcal{R}$ ). So there is a monoidal functor $F_{\mathcal{R}}: \mathcal{M} \rightarrow \mathcal{R}$. The fusion rule in $\mathcal{M}$ induces a fusion rule in $\mathcal{R}$. Thus, the bulk symmetry encoded in $\mathcal{N}$ becomes an algebraic symmetry in $\mathcal{R}$. However, the bulk excitations of $\mathcal{M}=Z_{1}(\mathcal{R})$ are more than that of $\mathcal{R}$. In this sense, the fusion rule of excitations in $\mathcal{M}$ gives rise to a bigger symmetry than that from the fusion rule of excitations in $\mathcal{R}$. This bigger symmetry corresponds to the categorical symmetry [19].
We know that $\mathrm{M}$ can have many boundaries (denoted by $\mathrm{C} \in \mathrm{TO}_{\mathrm{M}}^{n+1}$; see Definition 20). The excitations on the boundary are described by a fusion $n$-category $\mathcal{C}=\operatorname{Hom}(\mathrm{C}, \mathrm{C})=$ $\Omega \mathrm{C}$, which satisfies (see Proposition 19)

$$
\mathcal{M}=Z_{1}(\mathcal{C})
$$

As we move a bulk excitation in $\mathcal{M}$ to the boundary, it may become some boundary excitations in $\mathcal{C}$, or it may condense (i.e., become the trivial excitation in $\mathcal{C}$ ). So there is a forgetful functor $F_{\mathcal{C}}: \mathcal{M} \rightarrow \mathcal{C}$. Because some excitations in $\mathcal{M}$ are condensed on the boundary, we say the boundary spontaneously breaks part of the categorical symmetry $\mathcal{M}$. Different boundaries C's may spontaneously break different parts of the categorical symmetry $\mathrm{M}$, since the forgetful functor $F_{\mathcal{C}}$ may map different excitations in $M$ into the trivial excitations in $\mathcal{C}$ (i.e., condense different excitations of $\mathrm{M}$ on the boundary).

We see that all the boundaries have the same categorical symmetry $M$, if we view the boundary as a lattice boundary Hamiltonian. If we view the boundary as a state, then the categorical symmetry $M$ is spontaneously broken down to a smaller symmetry. The part of the categorical symmetry $M$, described by the excitations that condense on the boundary, is spontaneously broken. The smaller surviving symmetry is an algebraic higher symmetry. We know that the bulk fusion rule only induces the fusion rule for some boundary excitations (i.e., those in the image of the forgetful functor $F_{\mathcal{C}}$ ). Thus, the image of $F_{\mathcal{C}}$ is related to this algebraic higher symmetry-the unbroken part of the categorical symmetry.

One might expect the image of $F_{\mathcal{C}}$ to be the local fusion $n$-category that characterizes the algebraic higher symmetry in $\mathcal{C}$, but this impression is incorrect. The image of $F_{\mathcal{C}}$ may not even be a fusion $n$-category; i.e., there may not be an anomaly-free bulk topological order $\mathrm{M}$ whose boundary excitations realize the image of $F_{\mathcal{C}}$.

What is the algebraic higher symmetry in $\mathcal{C}$ (the unbroken part of the categorical symmetry $M$ )? First, such an algebraic higher symmetry must be described by a local fusion $n$-category $\mathcal{R}$. Since $\mathcal{R}$ is the unbroken part of the categorical symmetry $\mathrm{M}$, the corresponding categorical symmetry for $\mathcal{R}$ should be given by the same M. Mathematically, this means that $\operatorname{bulk}(\mathcal{R})=\mathrm{M}$. Since $\mathcal{R}$ is the algebraic higher symmetry in $\mathcal{C}, \mathcal{C}$ must contain all the charge objects of $\mathcal{R}$ as part of excitations in it. In other words, $\mathcal{R}$ can be embedded into $\mathcal{C}$; i.e., there exists an top-fully faithful functor $\iota: \mathcal{R} \stackrel{\iota}{\hookrightarrow} \mathcal{C}$. Here

Definition 29. Top-fully faithful means the functor is bijective when acting on top morphisms and is injective when acting on lower morphisms and on objects.

We know that the $\mathcal{R}$-symmetry can be explicitly broken, via the functors $\beta, \beta_{\mathcal{C}}$, which changes $\mathcal{R}$ to $n \mathcal{V}$ ec (see Definition 27) and changes $\mathcal{C}$ to $\underline{\mathcal{C}} . \underline{\mathcal{C}}$ describes the excitations in the anomaly-free topological order $\mathrm{C}_{0} \in \mathbb{T O}_{\mathrm{af}}^{n+1} \equiv(n+1) \mathcal{V e c}$ that are induced from $\mathrm{C}$ after we explicitly break the $\mathcal{R}$ symmetry in $\mathrm{C}$. We note that the excitations described by $\mathcal{C}$ contain both the topological excitations and the symmetrycharge excitations described by $\mathcal{R}$ (the charge objects of the algebraic higher symmetry). One may roughly understand $\mathcal{C}$ as " $\mathcal{C} / \mathcal{R}$;" i.e., "C $\bmod \mathcal{R}$ ". More precisely, $\underline{\mathcal{C}}$ is the pushout 
defined in the following diagram:

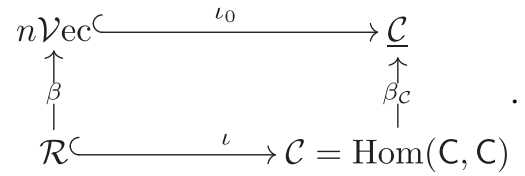

Moreover, the bulk of $\mathcal{R} \rightarrow n \mathcal{V e c}$ and $\mathcal{C} \rightarrow \mathcal{C}$ should coincide, which requires that $\gamma: Z_{1}(\mathcal{R}) \simeq Z_{1}(\mathcal{C})$ satisfies the condition as later illustrated in (155).

To summarize, the different boundaries of $\mathrm{M}$ all have the same categorical symmetry as a system, but the boundary may spontaneously break part of the categorical symmetry when viewed as a state. Because the charge objects of a categorical symmetry have nontrivial mutual statistics, the boundary that does not break the categorical symmetry $\mathrm{M}$ must be gapless $[19,60]$. For a gapped boundary, the categorical symmetry must be partially (and only partially) broken spontaneously. For the boundary $\mathrm{C}$ discussed above, the categorical symmetry is spontaneously broken down to the algebraic higher symmetry $\mathcal{R}$. We see the following:

Proposition 26. Categorical symmetries in $n$-dimensional space are fully characterized and classified by an $(n+1) \mathrm{d}$ anomaly-free topological orders $\mathrm{M}$.

A categorical symmetry $M$ may include several different anomaly-free algebraic higher symmetries $\mathcal{R}$, where $\mathcal{R}$ satisfies $\mathrm{M}=\operatorname{bulk}(\mathcal{R})$ (see Proposition 19).

A boundary of $\mathrm{M}$ is described by a boundary Hamiltonian. Such a Hamiltonian always has the full categorical symmetry M. The ground state of the boundary Hamiltonian, if gapped, is described by a boundary topological order $\mathrm{C}$ that satisfies $\operatorname{Bulk}(\mathrm{C})=\mathrm{M}$. For such a boundary ground state (i.e., the boundary topological order $\mathrm{C}$ ), the categorical symmetry is partially spontaneous broken, down to an algebraic higher symmetry $\mathcal{R}$ that satisfies (144). We say the categorical symmetry $\mathrm{M}$ contains the algebraic higher symmetry $\mathcal{R}$.

We would like to remark that for an $n$ d categorical symmetry, its corresponding topological order $\mathrm{M}$ in one higher dimension may have several different gapped boundaries with different unbroken algebraic higher symmetries. Thus, an categorical symmetry can contain several different algebraic higher symmetries. The gapped ground state of the boundary Hamiltonian must spontaneously break part of the categorical symmetry and can only spontaneously break part of the categorical symmetry. For example, as pointed out in Ref. [19], an $n$ d system with a 0 -symmetry described by a finite group $G$ (or a fusion $n$-category $n \mathcal{R} \operatorname{ep} G$ ) actually has a larger categorical symmetry. The categorical symmetry is characterized by a $G$-gauge theory $\mathrm{GT}_{G}^{n+1}=\operatorname{bulk}(n \mathcal{R}$ ep $G)$ in one higher dimension. The categorical symmetry include both the 0-symmetry $G$ (with $\mathcal{R}=n \mathcal{R} \operatorname{ep} G$ ) and an algebraic $(n-1)$-symmetry $G^{(n-1)}$ (with $\left.\mathcal{R}=n \mathcal{V} \mathrm{ec}_{G}\right)$.

\section{GAPPED LIQUID PHASES WITH ALGEBRAIC HIGHER SYMMETRY}

In Sec. VI, we discussed gapped liquid state with algebraic higher symmetry, which is a trivial symmetric product state. In this section, we are going to discuss gapped liquid phases with unbroken algebraic higher symmetry, which may have non- trivial topological orders. Those states are called SET states if the topological order is nontrivial (i.e., with long-range entanglement), or SPT states if the topological order is trivial (i.e., with short-range entanglement).

Let us first summarize some previous results in literature, which represent some systematic understanding of gapped liquid phases $[39,40]$ for boson and fermion systems with and without symmetry (but only for 0 -symmetry). In $1+1 \mathrm{D}$, all gapped phases are classified by $\left(G_{H}, G_{\Psi}, \omega_{2}\right)[88,89]$, where $G_{H}$ is the on-site symmetry group of the Hamiltonian, $G_{\Psi}$ is the symmetry group of the ground state $G_{\Psi} \subset G_{H}$, and $\omega_{2} \in H^{2}\left(G_{\Psi}, \mathbb{R} / \mathbb{Z}\right)$ is a group 2-cocycle for the unbroken symmetry group $G_{\Psi}$.

In $2+1 \mathrm{D}$, all gapped phases are classified (up to $E_{8}$ invertible topological orders and for a finite unitary onsite symmetry $\left.G_{H}\right)$ by $\left(G_{H}, \operatorname{Rep}\left(G_{\Psi}\right) \subset \mathcal{C} \subset \mathcal{M}\right)$ for bosonic systems and by $\left(G_{H}, \operatorname{sRep}\left(G_{\Psi}^{f}\right) \subset \mathcal{C} \subset \mathcal{M}\right)$ for fermionic systems $[45,46,90]$. Here $\operatorname{Rep}\left(G_{\Psi}\right)$ is the symmetric fusion category formed by representations of $G_{\Psi}$, and $\operatorname{sRep}\left(G_{\Psi}^{f}\right)$ is the symmetric fusion category formed by $\mathbb{Z}_{2}^{f}$-graded representations of $G_{\Psi}^{f}$, where $\mathbb{Z}_{2}^{f}$ is a center of $G_{\Psi}^{f}$. Also $\mathcal{C}$ is the braided fusion category of point-like excitations and $\mathcal{M}$ is a minimal modular extension $[45,46]$.

In $3+1 \mathrm{D}$, some gapped phases are liquid phases while others are nonliquid phases. The $3+1 \mathrm{D}$ gapped liquid phases without symmetry for bosonic systems (i.e., $3+1 \mathrm{D}$ bosonic topological orders) are classified by Dijkgraak-Witten theories if the point-like excitations are all bosons, by twisted 2-gauge theory with gauge 2-group $\mathcal{B}\left(G, Z_{2}\right)$ if some pointlike excitations are fermions and there are no Majorana zero modes, and by a special class of fusion 2-categories if some point-like excitations are fermions and there are Majorana zero modes at some triple-string intersections [28,67,91]. Compared with the classification of $3+1 \mathrm{D}$ SPT orders for bosonic [2,42] and fermioinc systems [50,51,53-56], this result suggests that all $3+1 \mathrm{D}$ gapped liquid phases (such as SET and SPT phases) for bosonic and fermionic systems with a finite unitary symmetry (including trivial symmetry, i.e., no symmetry) are classified by partially gauging the symmetry of the bosonic/fermionic SPT orders [28].

We see that the previous approaches are quite different for different dimensions and are only for 0-symmetries. In this section, we describe a classification that works for all dimensions. We also generalize the 0 -symmetry in the above results to algebraic higher symmetry.

\section{A. Partially characterize a symmetric gapped liquid phase using a pair of fusion higher categories}

The classification of gapped liquid phases with algebraic higher symmetry is quite complicated. In this section, we state a simple partial result to identify the difficulties and the issues in the classification.

For a gapped liquid state with unbroken algebraic higher symmetry $\mathcal{R}$, there are point-like, string-like, etc. excitations that correspond to the charge objects of the symmetry. Those charge objects are described by the representation category $\mathcal{R}$. In general, the gapped liquid state also has extra point-like, string-like, etc. excitations that are beyond those described by 
the local fusion higher category $\mathcal{R}$, so the total excitations are described by a bigger fusion higher category $\mathcal{C}$ that includes $\mathcal{R}$. This leads to the following result:

Proposition 27. An $n$ d anomaly-free gapped liquid phase with an unbroken algebraic higher symmetry described by a fusion $n$-category $\mathcal{R}$ is fully described by a potentially anomalous topological order $C$ (see Sec. III F). The excitations of $C$ are described by a fusion $n$-category $\mathcal{C}=\Omega \mathrm{C}$ which admits a top-fully faithful functor $\mathcal{R} \stackrel{\iota}{\hookrightarrow} \mathcal{C}$. Thus, we can use the data $\mathcal{R} \stackrel{\iota}{\hookrightarrow} \mathcal{C}$ to partially classify the gapped liquid phase with algebraic higher symmetry $\mathcal{R}$.

One way to see the above result is to note that stacking a trivial symmetric state $\mathcal{R}$ and the symmetric topological order $\mathcal{C}$ together give rise to a fusion $n$-category $\mathcal{R} \otimes \mathcal{C}$, if there is no coupling between the trivial symmetric state $\mathcal{R}$ and the symmetric topological order $\mathcal{C}$. The $\mathcal{R} \otimes \mathcal{C}$ state has a larger algebraic higher symmetry $\mathcal{R} \otimes \mathcal{R}$, one from the trivial symmetric state $\mathcal{R}$ and the other from the symmetric topological order $\mathcal{C}$. However, we can add the so-called "symmetric interactions" between $\mathcal{R}$ and $\mathcal{C}$ to reduce the $\mathcal{R} \otimes \mathcal{R}$ symmetry to the original symmetry $\mathcal{R}$. The stacking with such symmetric interactions, which preserves the diagonal $\mathcal{R}$ symmetry but break the other symmetries, is denoted by $\otimes_{\mathcal{R}}$ and $\mathcal{R} \otimes_{\mathcal{R}} \mathcal{R}=\mathcal{R}$. Including the "symmetric interactions" is similar to adding the symmetry-breaking morphisms in our definition of local fusion higher category (see Definition 27). Such a process can also be realized by a condensation of excitations. Since $\mathcal{R}$ is local, the condensation does not confine any excitations in $\mathcal{R}$, and all the excitations in $\mathcal{R}$ become excitations in $\mathcal{R} \otimes_{\mathcal{R}} \mathcal{C}$. Physically, we require that $\mathcal{R} \otimes_{\mathcal{R}} \mathcal{C}=\mathcal{C}$. Therefore, all the excitations in $\mathcal{R}$ become excitations in $\mathcal{C}$. Thus, there is a functor $\mathcal{R} \stackrel{\iota}{\hookrightarrow} \mathcal{C}$, which is faithful (i.e., injective) at each level of morphisms and objects. Since both $\mathcal{R}$ and $\mathcal{C}$ have the same algebraic higher symmetry $\mathcal{R}$, the allowed local symmetric operators are the same. Thus, the faithful functor $\mathcal{R} \stackrel{\iota}{\hookrightarrow} \mathcal{C}$ is fully faithful (i.e., bijective) at the top morphisms (which correspond to the local symmetric operators).

Does every pair of fusion $n$-categories $(\mathcal{R}, \mathcal{C})$ satisfying $\mathcal{R} \stackrel{\iota}{\hookrightarrow} \mathcal{C}$ describe an anomaly-free topological order with an algebraic higher symmetry? The answer is no, as implied by some counterexamples when $\mathcal{R}$ describes a 0 -symmetry $[45,46]$. If the pair $(\mathcal{R}, \mathcal{C})$ does describe a symmetric topological order, does it uniquely describe the symmetric topological order? The answer is also no. For example, a pair of fusion $n$-categories $(\mathcal{R}, \mathcal{R})$ can describe a symmetric trivial product state. The same pair $(\mathcal{R}, \mathcal{R})$ can also describe a SPT state of the same symmetry. This is because, as we mentioned before, the fusion $n$-category only describes the excitations which do not contain all the information of a topological order and cannot distinguish different invertible topological orders. In our case here, the pair $(\mathcal{R}, \mathcal{R})$ cannot distinguish symmetric trivial product state from nontrivial SPT state with the same anomaly-free algebraic higher symmetry.

However, the $\mathcal{R} \stackrel{\iota}{\hookrightarrow} \mathcal{C}$ description does not miss much. In the following, we try to understand which pairs $\mathcal{R} \stackrel{\iota}{\hookrightarrow} \mathcal{C}$ can describe anomaly-free topological orders with an algebraic higher symmetry. We also try to seek additional information beyond $\mathcal{R} \stackrel{\iota}{\hookrightarrow} \mathcal{C}$ to fully characterize a symmetric topological order. One way to achieve both goals is to use the notion of categorical symmetries described in Ref. [19] and Sec. VIE, which is a holographical way to view a symmetry. This way to view a symmetry is most suitable for algebraic higher symmetries. It gives an even more general perspective about algebraic higher symmetries. So in the next section, we first study gapped liquid phases in a bosonic system with a categorical symmetry.

\section{B. Classification of gapped liquid phases in bosonic systems with a categorical symmetry}

Here, we will address the difficulties and the issues identified in the last section via a holographic approach. We will first consider a modified problem: classification of gapped liquid phases in bosonic systems with a categorical symmetry. Note that the gapped liquid phases do not have the full categorical symmetry, since gapped phases always partially break the categorical symmetry spontaneously. After this discussion, we identify a key difficulty in the classification.

Let us consider an $n d$ bosonic lattice Hamiltonian with an algebraic higher symmetry $\mathcal{R}$. Such a lattice Hamiltonian can also be regarded as having a categorical symmetry characterized by an anomaly-free topological order $\mathrm{M}=\operatorname{bulk}(\mathcal{R})$ in one higher dimension, since algebraic higher symmetry $\mathcal{R}$ and categorical symmetry $\mathrm{M}=\operatorname{bulk}(\mathcal{R})$ are holo-equivalent. What are the gapped liquid phases that have this categorical symmetry M? The answer is that there are no such phases, since gapped phases in $n$ d lattice Hamiltonians with a nontrivial categorical symmetry $\mathrm{M}$ must partially break and only partially break the categorical symmetry spontaneously $[19,60]$. This is because a gapped phase in an $n$ d bosonic lattice Hamiltonian with a categorical symmetry $M$ corresponds to a gapped boundary of a $(n+1) \mathrm{d}$ anomaly-free topological order M. The gapped boundary comes from the condensation of some of the excitations in $M$, and thus part of the categorical symmetry is spontaneously broken. In fact, the condensing excitations form a Lagrangian condensable algebra, which corresponds to spontaneously breaking part of the categorical symmetry. Also, the excitations that can condense (i.e., those in the Lagrangian condensable algebra) must have trivial mutual statistics between them. Therefore, we cannot condense all the excitations in $\mathrm{M}$ simultaneously. This is why we cannot completely break a categorical symmetry spontaneously. (Certainly, we can always partially or completely break a categorical symmetry explicitly.) This picture leads to the following result [see Fig. 8(a)]:

Proposition 28. For $n$ d bosonic lattice Hamiltonians with a categorical symmetry $\mathrm{M}$, their gapped liquid phases are classified by the gapped boundaries of $(n+1) \mathrm{d}$ anomaly-free topological orders M. In other words, the gapped liquid phases are classified by (potentially anomalous) topological orders C's (objects in $\mathrm{TO}_{\mathrm{M}}^{n+1}$ 's; see Sec. III F) satisfying the condition

$$
\mathrm{M}=\operatorname{Bulk}(\mathrm{C}) .
$$

In light of Propositions 15 and 16, the above result implies the following [see Fig. 8(a)]: 
Proposition 29. For $n$ d bosonic lattice Hamiltonians with a categorical symmetry $\mathrm{M}$, the excitations in their gapped liquid phases are described by fusion $n$-categories $\mathcal{C}$ such that

$$
\operatorname{bulk}(\mathcal{C})=\mathrm{M} \text {. }
$$

For every fusion $n$-category $\mathcal{C}$ satisfying bulk $(\mathcal{C})=\mathrm{M}$, there are one or more gapped liquid phases, $\mathrm{C}$ 's, to realize it: $\mathcal{C}=$ $\Omega \mathrm{C}$.

Let us remark on the second part of the above result. Given a fusion $n$-category $\mathcal{C}$ describing the excitations in a gapped phase of $n$ d bosonic lattice Hamiltonian with categorical symmetry $\mathrm{M}$, do we have another gapped phase of another $n \mathrm{~d}$ bosonic lattice Hamiltonian with categorical symmetry M that also has excitations described by $\mathcal{C}$ ? In general, the answer is yes. This is because two gapped phases, differing in the stacking of invertible topological orders or SPT orders, have the same set of excitations.

Proposition 30. The gapped liquid phases in $n \mathrm{~d}$ bosonic lattice Hamiltonians with a categorical symmetry $M$ are partially classified by the fusion $n$-categories $\mathcal{C}$ that satisfy $\operatorname{bulk}(\mathcal{C})=\mathrm{M}$.

Here partially means that the classification is one-to-many: The same fusion $n$-category $\mathcal{C}$ may correspond to several different gapped liquid phases, C's, of systems with the categorical symmetry $\mathrm{M}$. As we have mentioned before, the gapped liquid phases must break only part of the categorical symmetry M spontaneously.

To get a full one-to-one classification, we need to find extra information beyond the excitations, i.e., the fusion $n$-category $\mathcal{C}$, to characterize the gapped liquid states. One way to get extra information is to study the boundaries of the gapped liquid states. This will be done later.

Here, we consider another type of extra information. As we have mentioned above, stacking invertible topological orders or SPT orders to a gapped phase does not change the excitations. Let us consider a boundary of $\mathrm{M}$ with excitations $\mathcal{C}$ (see Fig. 8). We can use the trivial excitations in $M$ to form an $n$ d invertible topological order, which is a domain wall in $M$. We can also use the topological excitations in $M$ to form an $n$ d SPT order, which is also a domain wall in M. The protecting symmetry of the SPT order comes from the fusion rule (the conservation law) of the topological excitations that form the SPT order. Both kinds of domain walls are invertible domain walls. There are also invertible domain walls that correspond to braided automorphisms of the braided fusion $n$-category $\mathcal{M}=\Omega^{2} \mathrm{M}$ describing the excitations in $\mathrm{M}$. In fact, each invertible domain wall $\hat{\gamma}$ corresponds to a braided automorphism $\gamma$ of $\mathcal{M}$ (see Fig. 17). Thus, stacking an invertible domain wall $\hat{\gamma}$ to the boundary $\mathcal{C}$ give us a boundary $(\mathcal{C}, \hat{\gamma})$ that is related to the boundary $\mathcal{C}$ via an automorphism $\gamma$, so the two boundaries are described by fusion $n$-categories that are equivalent to $\mathcal{C}$. However, the boundaries $(\mathcal{C}, \hat{\gamma})$, with different invertible domain walls $\hat{\gamma}$, may correspond to different boundary phases, i.e., inequivalent C's. We conjecture that all different boundary phases, C's, with sets of boundary excitations equivalent to $\mathcal{C}$ can be obtained this way: $\mathrm{C}=(\mathcal{C}, \hat{\gamma})$. This leads to the following result [see Fig. 8(b)]:

Proposition 31. For $n$ d bosonic lattice Hamiltonians with a categorical symmetry $\mathrm{M}$, their gapped liquid phases are classified by a pair $(\mathcal{C}, \hat{\gamma})$, where $\mathcal{C}$ is a fusion $n$-category $\mathcal{C}$

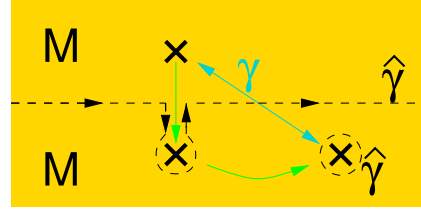

FIG. 17. Consider a topological order $\mathrm{M}$ separated by a invertible domain wall $\hat{\gamma}$. Moving an excitation (with codimension-2 or higher) across the invertible domain wall $\hat{\gamma}$ (with codimension-1) induces an braided automorphism $\gamma$ of the braided fusion higher category $\mathcal{M}=\Omega^{2} \mathrm{M}: \mathcal{M} \stackrel{\nu}{\simeq} \mathcal{M}$.

that satisfies bulk $(\mathcal{C}) \stackrel{\hat{d}}{\simeq} \mathrm{M}$, and $\hat{\gamma}$ is an invertible domain wall between $\operatorname{bulk}(\mathcal{C})$ and $\mathrm{M}$.

The possible invertible domain wall $\hat{\gamma}$ is, of course, not unique. However, when we are considering gapped liquid phases with an algebraic higher symmetry $\mathcal{R}$ [instead of gapped liquid phases that may spontaneously break the categorical symmetry $M=\operatorname{bulk}(\mathcal{R})$ ], the different invertible domain walls may have different physical meanings with respect to $\mathcal{R}$. An invertible domain wall may either preserve the algebraic higher symmetry $\mathcal{R}$, or (partially or completely) break $\mathcal{R}$. To classify gapped liquid phases with an algebraic higher symmetry $\mathcal{R}$, we need to select $\hat{\gamma}$ 's that preserve the algebraic symmetry $\mathcal{R}$.

How to select $\hat{\gamma}$ 's is a key difficulty in the classification. In the next section, we give several (hopefully equivalent) criteria when an invertible domain wall $\hat{\gamma}$ preserves an algebraic symmetry $\mathcal{R}$.

\section{Classification of SET orders and SPT orders with an algebraic higher symmetry}

\section{A simple result}

Let us first give a simple partial result by ignoring the invertible domain wall $\hat{\gamma}$ (i.e., by overlooking the key difficulty). Given an algebraic higher symmetry $\mathcal{R}$, there is an $(n+1) \mathrm{d}$ anomaly-free topological order $\mathrm{M}=\operatorname{bulk}(\mathcal{R})$ (i.e., the holo-equivalent categorical symmetry) that has one boundary with excitations described by $\mathcal{R}=\Omega \mathrm{R}$. The boundary topological order $\mathrm{R}$ corresponds to a trivial product state with the algebraic higher symmetry $\mathcal{R}$. (More precisely, the trivial product state with the algebraic higher symmetry $\mathcal{R}$, plus its excitations, when restricted to the symmetric sub-Hilbert space $\mathcal{V}_{\text {symm }}$, correspond to the boundary topological order $R$.)

Now consider another boundary $C$ of $M$, with the excitations described by a fusion $n$-category $\mathcal{C}=\Omega \mathrm{C}$ ). However, the boundary $\mathrm{C}$ may spontaneously break the algebraic higher symmetry $\mathcal{R}$. Here we would like to classify gapped liquid phases, C's, that do not spontaneously break the algebraic higher symmetry $\mathcal{R}$. To do so, we just need to select C's such that the excitations $\mathcal{C}$ contain $\mathcal{R}$. We believe that all the anomaly-free topological orders with the algebraic higher symmetry $\mathcal{R}$ can be viewed in this way. If we replace $C$ by $\mathcal{C}$ to get a partial classification, we obtain (see Fig. 18) the following:

Proposition 32. Anomaly-free gapped liquid phases with an algebraic higher symmetry $\mathcal{R}$ in $n$-dimensional space are partially classified by fusion $n$-categories $\mathcal{C}$ that satisfy 


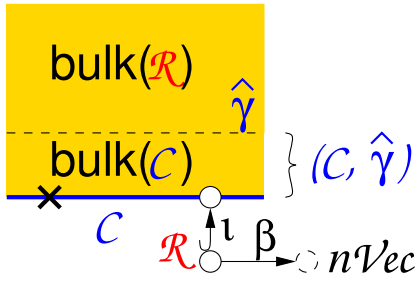

FIG. 18. A gapped liquid phase with an algebraic higher symmetry $\mathcal{R}$ can be characterized by a pair $(\mathcal{C}, \hat{\gamma})$, or more precisely, by $\mathcal{C} \underset{\text { bulk }(\mathcal{C})}{\otimes} \hat{\gamma} \underset{\text { bulk }(\mathcal{R})}{\otimes}$, where $\hat{\gamma}$ is an invertible domain wall between $\operatorname{bulk}(\mathcal{R})$ and $\operatorname{bulk}(\mathcal{C})$ : $\operatorname{bulk}(\mathcal{R}) \stackrel{\hat{\mathcal{D}}}{\simeq} \operatorname{bulk}(\mathcal{C})$. The fusion $n$-category $\mathcal{C}$ on the boundary describes the excitations on top of the gapped liquid state, which include $\mathcal{R}$; i.e., $\mathcal{R} \stackrel{\iota}{\hookrightarrow}$. Since $\mathcal{R}$ is local, we also has a fiber functor $\beta: \mathcal{R} \rightarrow n \mathcal{V}$ ec. Not every invertible domain wall can be included. Finding the condition to select the proper invertible domain walls $\hat{\gamma}$ is the key to classify gapped liquid phases with symmetry $\mathcal{R}$. The cross is a generic excitation in $\mathcal{C}$. The white circles are $\mathcal{R}$-charge objects in $\mathcal{C}$.

$\operatorname{bulk}(\mathcal{C}) \simeq \operatorname{bulk}(\mathcal{R})$ and admit a top-fully faithful functor $\mathcal{R} \stackrel{\iota}{\hookrightarrow} \mathcal{C}$.

Here $\operatorname{bulk}(\mathcal{C}) \simeq \operatorname{bulk}(\mathcal{R})$ means that the two bulk topological orders, bulk $(\mathcal{C})$ and $\operatorname{bulk}(\mathcal{R})$, are connected by an invertible domain wall (see Fig. 17). We used a more relaxed condition, just requiring bulk $(\mathcal{C})$ and $\operatorname{bulk}(\mathcal{R})$ to be equivalent rather than equal. This is because we are not considering different boundaries of a fixed bulk. Here we are considering different boundaries and their bulks, and hoping the bulks are the same, but when we compare two bulks to see if they are the "same," the best we can do is to see whether they are equivalent, i.e., whether they are connected by an invertible domain wall.

We would like to point out that the above $\mathcal{C}$ classifies the excitations in the anomaly-free topological order $\mathrm{C}$ with an algebraic higher symmetry $\mathcal{R}$ (i.e., $\mathrm{C}$ can be realized by a bosonic lattice model in the same dimensions with the symmetry $\mathcal{R}$ ). We know that topological orders differ by invertible gapped liquids have the same excitations. Thus, the above $\mathcal{C}$ 's cannot distinguish different invertible gapped liquids, i.e., different invertible topological orders and SPT orders. The above $\mathcal{C}$ 's only classify anomaly-free topological orders with the algebraic higher symmetry $\mathcal{R}$, up to invertible topological orders and SPT orders for symmetry $\mathcal{R}$.

To obtain a more complete classification, i.e., to include the SPT orders with symmetry $\mathcal{R}$, we should include the invertible domain walls $\hat{\gamma}$ : $\operatorname{bulk}(\mathcal{R}) \simeq \operatorname{bulk}(\mathcal{C})$ as our data as we discussed in Sec. VII B (see Fig. 18):

Proposal 1. Bosonic anomaly-free gapped liquid phases in $n$-dimensional space with an anomaly-free algebraic higher symmetry $\mathcal{R}$ are classified by data $(\mathcal{C}, \iota: \mathcal{R} \hookrightarrow \mathcal{C}, \hat{\gamma}$ : $\operatorname{bulk}(\mathcal{R}) \simeq \operatorname{bulk}(\mathcal{C})$, where $\mathcal{C}$ is a fusion $n$-category that includes $\mathcal{R}$ (i.e., $\iota: \mathcal{R} \hookrightarrow \mathcal{C}$ is a top-fully faithful functor; see Proposition 27), and $\hat{\gamma}: \operatorname{bulk}(\mathcal{R}) \simeq \operatorname{bulk}(\mathcal{C})$ is a invertible domain wall between $\operatorname{bulk}(\mathcal{R})$ and $\operatorname{bulk}(\mathcal{C})$.

However, the above proposal is incorrect. We cannot use an arbitrary invertible domain wall $\hat{\gamma}: \operatorname{bulk}(\mathcal{R}) \simeq \operatorname{bulk}(\mathcal{C})$. The reason is that $\mathcal{C}$ contains the symmetry $\mathcal{R}$ via the embed-

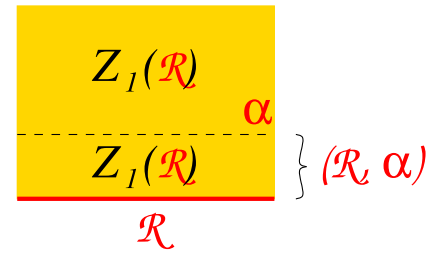

FIG. 19. Similar to Fig. 18, with $\mathcal{C}=\mathcal{R}$. An SPT state is characterized by a pair $(\mathcal{R}, \alpha)$, or more precisely, by the stacking of $\mathcal{R}$ and $\alpha$ through the bulk $Z_{1}(\mathcal{R}): \mathcal{R} \otimes \alpha \otimes \otimes$. Here $\alpha$ is a braided automorphism of $Z_{1}(\mathcal{R})$. Not every automorphism can be included. Finding the condition to select the proper automorphisms $\alpha$ is the key to classify $\mathcal{R}$-SPT orders.

ding $\iota: \mathcal{R} \hookrightarrow \mathcal{C}$. bulk $(\mathcal{C})$ also contains the symmetry $\mathcal{R}$ via the forgetful functor $F_{\mathcal{C}}: \Omega^{2} \operatorname{bulk}(\mathcal{C})=Z_{1}(\mathcal{C}) \rightarrow \mathcal{C}$. bulk $(\mathcal{R})$ also contains the symmetry $\mathcal{R}$ via the forgetful functor $F_{\mathcal{R}}$ : $\Omega^{2}$ bulk $(\mathcal{R})=Z_{1}(\mathcal{R}) \rightarrow \mathcal{R}$. If we allow an arbitrary invertible domain wall $\hat{\gamma}$ which induces an arbitrary braided equivalence $\gamma: Z_{1}(\mathcal{R}) \simeq Z_{1}(\mathcal{C})$, then the $\mathcal{R}$ symmetry contained in $Z_{1}(\mathcal{C})$ and $Z_{1}(\mathcal{C})$ may not be compatible (i.e., may not be matched by $\gamma$ ). Thus, the key is to find proper conditions to select proper $\hat{\gamma}$ 's. In the following, we describe several, hopefully equivalent, ways to do so.

When $\mathcal{C}=\mathcal{R}$, the above reduces to a classification of SPT phases with algebraic higher symmetry $\mathcal{R}$ via a pair $(\mathcal{R}, \alpha)$, where $\alpha$ is an automorphism of $Z_{1}(\mathcal{R})$ (see Fig. 19). To describe this classification of SPT phases in more detail, we like to remark that the map from the invertible domain walls $\hat{\alpha}$ in $\operatorname{bulk}(\mathcal{R})$ to the braided automorphisms $\alpha$ of $\Omega^{2} \operatorname{bulk}(\mathcal{R})=$ $Z_{1}(\mathcal{R})$ may not be one to one. We conjecture the following:

Conjecture 1. The kernel of the map $\hat{\alpha} \rightarrow \alpha$ is the set of invertible domain walls in $\operatorname{bulk}(\mathcal{R})$ that correspond to invertible topological orders formed by trivial excitations in $\Omega^{2} \operatorname{bulk}(\mathcal{R})=Z_{1}(\mathcal{R})$.

This conjecture is based on the belief that any properties of excitations $Z_{1}(\mathcal{R})$ cannot see invertible topological orders.

Because the classification of SPT phases does not include invertible topological orders, we can replace the invertible domain walls $\hat{\alpha}$ by the braided automorphisms $\alpha$. This allows us to obtain the following proposal (see Fig. 19):

Proposal 2. Bosonic SPT phases in $n$-dimensional space with an anomaly-free algebraic higher symmetry $\mathcal{R}$ are classified by the braided automorphisms $\alpha$ of $Z_{1}(\mathcal{R})$.

Again, the above proposal is not correct since some braided automorphisms $\alpha$ may break the symmetry $\mathcal{R}$ [i.e., change the symmetry $\mathcal{R}$ contained in $Z_{1}(\mathcal{R})$ ]. To make it correct, we need to find proper conditions to select proper braided automorphisms $\alpha$ that do not break the symmetry $\mathcal{R}$.

The above discussions reveal the key difficulty in classifying gapped liquid phases with an algebraic higher symmetry. In the next few subsections, we propose several approaches to address this issue, which leads to several, hopefully equivalent, classification results.

\section{A classification assuming $\mathcal{R}$ to be symmetric}

When $\mathcal{R}$ is symmetric, it can be lifted to the bulk $Z_{1}(\mathcal{R})$ via a canonical braided embedding $\iota_{\mathcal{R}}: \mathcal{R} \hookrightarrow Z_{1}(\mathcal{R})$. In this 


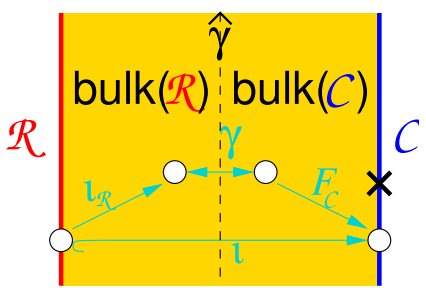

FIG. 20. Similar to Fig. 18, but now we assume $\mathcal{R}$ to be symmetric. In this case, the $\mathcal{R}$-charges in $\mathcal{R}$ can be lifted into the bulk via the embedding $\iota_{\mathcal{R}}$. The equivalence $\gamma$ should be compatible with the lifting $\iota_{\mathcal{R}}$ of $\mathcal{R}$, the embedding $\iota: \mathcal{R} \hookrightarrow \mathcal{C}$, and the bulk-to-boundary functor $F_{\mathcal{R}}$.

case, we have a simple criteria for $\gamma$ to make the two $\mathcal{R}$ symmetries in $\mathcal{C}$ and $Z_{1}(\mathcal{R})$ compatible (i.e., to preserve the $\mathcal{R}$ symmetry; see Fig. 20):

Proposition 33. Anomaly-free gapped liquid phases in $n$ dimensional space with an anomaly-free algebraic higher symmetry $\mathcal{R}$ (which is assumed to be symmetric) are classified by data $(\mathcal{C}, \iota: \mathcal{R} \hookrightarrow \mathcal{C}, \hat{\gamma}: \operatorname{bulk}(\mathcal{R}) \simeq \operatorname{bulk}(\mathcal{C}))$, where $\mathcal{C}$ is a fusion $n$-category that includes $\mathcal{R}$ (i.e., $\iota: \mathcal{R} \hookrightarrow \mathcal{C}$ is a top-fully faithful functor), and $\hat{\gamma}$ is an invertible domain wall rendering the following diagram commutative (up to a natural isomorphism),

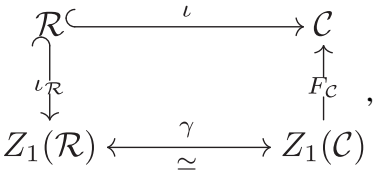

where $\gamma$ is the braided equivalence $\gamma: Z_{1}(\mathcal{R}) \simeq Z_{1}(\mathcal{C})$ induced by the invertible domain wall $\hat{\gamma}: \operatorname{bulk}(\mathcal{R}) \simeq \operatorname{bulk}(\mathcal{C})$ and $F_{\mathcal{C}}: Z_{1}(\mathcal{C}) \rightarrow \mathcal{C}$ is the forgetful functor.

Reference [68] proposed this result in a slightly different manner. An embedding $\iota_{\mathcal{C}}: \mathcal{R} \hookrightarrow Z_{1}(\mathcal{C})$ is considered as the data for gapped liquid phases instead of $\iota: \mathcal{R} \hookrightarrow \mathcal{C}$, and it is required that $F_{\mathcal{C}} \circ l_{\mathcal{C}}: \mathcal{R} \rightarrow \mathcal{C}$ is an embedding, thus reproducing the data $\iota$. Then, (147) is replaced by (see Fig. 21)

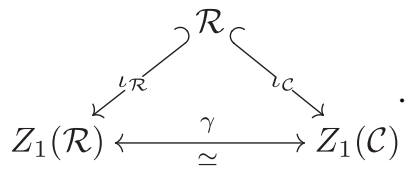

When $\mathcal{C}=\mathcal{R}$, the above result reduces to a classification of SPT orders with symmetry $\mathcal{R}$ (see Fig. 22):

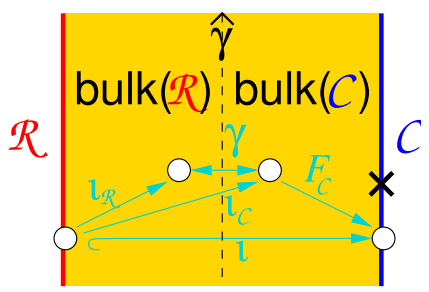

FIG. 21. The graphic representation of (148) which is equivalent to Fig. 20. We assume $\mathcal{R}$ to be symmetric. In this case, the $\mathcal{R}$-charges in $\mathcal{R}$ and in $\mathcal{C}$ can be lifted into the bulk via the embedding $\iota_{\mathcal{R}}$ and $\iota_{\mathcal{C}}$.

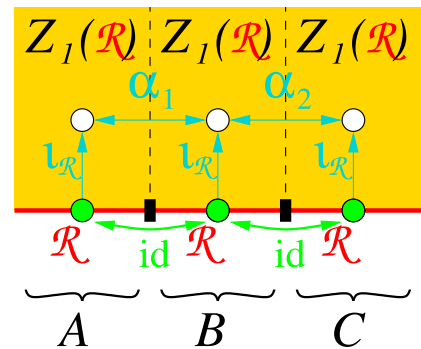

FIG. 22. Three SPT phases $A, B, C$ with symmetry $\mathcal{R}$. $A$ and $B$ differ by an invertible domain wall characterized by the equivalence $\alpha_{1}$ [satisfying (149)]. $B$ and $C$ differ by an invertible domain wall $\alpha_{2}$. Then $A$ and $C$ differ by an invertible domain wall characterized by the composite equivalences $\alpha_{1} \circ \alpha_{2}$. We see that the SPT phases are classified by the invertible domain walls, i.e., by the equivalences $\alpha: Z_{1}(\mathcal{R}) \stackrel{\alpha}{\simeq} Z_{1}(\mathcal{R})$ satisfying (149).

Proposition 34. SPT phases in $n$-dimensional space with an anomaly-free algebraic higher symmetry $\mathcal{R}$ (which is assumed to be symmetric) are classified by data $(\mathcal{R}, \alpha)$, where $\alpha: Z_{1}(\mathcal{R}) \simeq Z_{1}(\mathcal{R})$ is a braided equivalence rendering the following diagram commutative (up to a natural isomorphism):

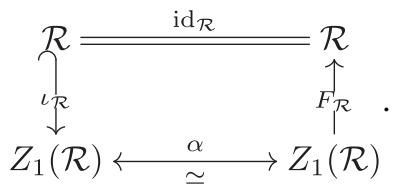

Remark 6. Note that $F_{\mathcal{R}} \circ \alpha \circ \iota_{\mathcal{R}}=\mathrm{id}_{\mathcal{R}}=F_{\mathcal{R}} \circ \iota_{\mathcal{R}}$ is a central functor, where the central structure comes from the symmetric structure of $\mathcal{R}$. By the universal property of center [27], (149) is equivalent to

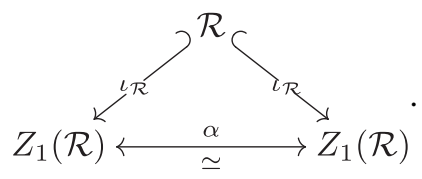

\section{First version of general classification}

Now we discuss a classification for more general algebraic higher symmetry where $\mathcal{R}$ may not be symmetric. To do so, we need a very different approach. Let us first consider the classification of bosonic SPT orders with an algebraic higher symmetry $\mathcal{R}$ in $n$-dimensional space. Those SPT orders all have excitations described by the same local fusion $n$-category $\mathcal{R}$. To distinguish different SPT orders, we need to include extra information beyond $\mathcal{R}$, and to use pairs $(\mathcal{R}, \alpha)$ to describe the SPT orders, where $\alpha$ is an automorphism of $Z_{1}(\mathcal{R})$. To identify the proper $\alpha$ 's, we notice that the physical way to distinguish different SPT orders is to include the boundary of a SPT state. Here we consider the canonical boundary that spontaneously breaks all the symmetry $\mathcal{R}$.

In the following, we develop a theory for the canonical boundary that breaks all the symmetry $\mathcal{R}$, using a holographic point of view of the symmetry $\mathcal{R}$, i.e., using a topological order with excitations $Z_{1}(\mathcal{R})$ in one higher dimension to describe the symmetry $\mathcal{R}$. In other words, we need to use the holographic point of view to describe the boundary that breaks all the symmetry $\mathcal{R}$. Such a symmetry-breaking boundary also 

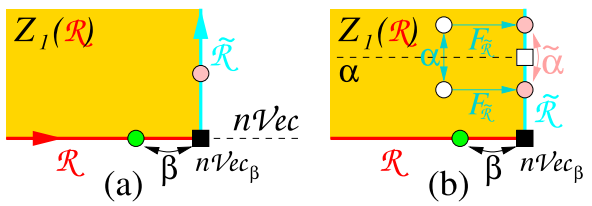

FIG. 23. (a) A SPT state with symmetry $\mathcal{R}$ (the red line) can be viewed as a gapped boundary of a topological order in one higher dimension with excitations $Z_{1}(\mathcal{R})$. The boundary $n \mathcal{V} \mathrm{ec}_{\beta}$ of SPT state $\mathcal{R}$ that breaks all the $\mathcal{R}$-symmetry also has a bulk, which can be viewed as a gapped boundary of $Z_{1}(\mathcal{R})$ for the dual symmetry $\widetilde{\mathcal{R}}$. (b) The automorphism $\alpha$ of $Z_{1}(\mathcal{R})$ corresponds to an invertible domain wall in the bulk (the dashed line), which also has an invertible boundary (the white square). The boundary-bulk relation between $Z_{1}(\mathcal{R})$ and $\widetilde{\mathcal{R}}$ is described by the bulk-to-boundary functor $F_{\widetilde{\mathcal{R}}}$. Such a boundary-bulk relation is preserved by the automorphisms $\alpha, \widetilde{\alpha}$, which classify the $\mathcal{R}$-SPT orders.

has a bulk in one higher dimension. Such a bulk has a different set of excitations described by another local fusion $n$-category, denoted as $\widetilde{\mathcal{R}}$. In fact, $\widetilde{\mathcal{R}}$ can be viewed as another gapped boundary of the bulk $Z_{1}(\mathcal{R})$ [see Fig. 23(a)], and therefore $Z_{1}(\mathcal{R}) \simeq Z_{1}(\widetilde{\mathcal{R}}) . \widetilde{\mathcal{R}}$ is nothing but the local fusion $n$-category that describes the dual symmetry of $\mathcal{R}$ (see Sec. IIE). For example, when $\mathcal{R}=n \mathcal{R} \operatorname{ep} G$, the symmetry is the 0 -symmetry described by the group $G$. The dual symmetry is an algebraic higher symmetry described by $\widetilde{\mathcal{R}}=n \mathcal{V e c}_{G}$.

We know that the bulk $Z_{1}(\mathcal{R})$ and the boundaries, when viewed as systems, have a categorical symmetry $Z_{1}(\mathcal{R})$ that includes both the symmetry $\mathcal{R}$ and the dual symmetry $\widetilde{\mathcal{R}}$. The boundary $\mathcal{R}$ in Fig. 23 has the symmetry $\mathcal{R}$ but spontaneously breaks the dual symmetry $\widetilde{\mathcal{R}}$, while the boundary $\widetilde{\mathcal{R}}$ has the dual symmetry $\widetilde{\mathcal{R}}$ but spontaneously breaks the symmetry $\mathcal{R}$. The intersection $n \mathcal{V} \mathrm{ec}_{\beta}$ of the two boundaries breaks both the symmetry $\mathcal{R}$ and the dual symmetry $\widetilde{\mathcal{R}}$ [see Fig. 23(a)].

The "bulk" of the canonical boundary $n \mathcal{V} \mathrm{ec}_{\beta}$ of $\mathcal{R}\left(n \mathcal{V} \mathrm{ec}_{\beta}\right.$ is the same $n$-category as $n \mathcal{V}$ ec with $\mathcal{R}$-module structure induced by $\beta: \mathcal{R} \rightarrow n \mathcal{V e c}$ ), which is also a "boundary" of the bulk of $\mathcal{R}$, gives us the criteria when the automorphism $\alpha: Z_{1}(\mathcal{R}) \simeq Z_{1}(\mathcal{R})$ preserves the symmetry $\mathcal{R}$ and thus represents an $\mathcal{R}$ SPT order [see Fig. 23(a)]. To identify the proper automorphisms, we note that $\alpha$ can be viewed as an invertible domain wall in the bulk $Z_{1}(\mathcal{R})$ [see Fig. 23(b)]. Such an invertible domain wall has a boundary on the boundary $\widetilde{\mathcal{R}}$ [the white square in Fig. 23(b)]. Since the difference between SPT orders are invertible, the boundary of the invertible domain wall should also be invertible. This motivates us to conjecture that the boundary of the invertible domain wall $\alpha$ corresponds to an automorphism $\widetilde{\alpha}$ of $\widetilde{\mathcal{R}}$. The automorphisms $\alpha$ for the bulk $Z_{1}(\mathcal{R})$ and $\widetilde{\alpha}$ for the boundary $\widetilde{\mathcal{R}}$ should preserve the whole structure of $\mathcal{R}$ and its boundary $n \mathcal{V} \mathrm{ec}_{\beta}$ [the red line and the black box in Fig. 23(b)]. This can be achieved by requiring $\alpha, \widetilde{\alpha}$ to preserve the bulk-boundary relation described by the bulk to boundary functor $F_{\widetilde{\mathcal{R}}}: Z_{1}(\mathcal{R}) \rightarrow \widetilde{\mathcal{R}}$. This leads to the following result:

Proposition 35. Bosonic SPT orders with an anomalyfree algebraic higher symmetry $\mathcal{R}$ in $n$-dimensional space are classified by braided equivalence $\alpha: Z_{1}(\mathcal{R}) \simeq Z_{1}(\mathcal{R})$ and monoidal equivalence $\widetilde{\alpha}: \widetilde{\mathcal{R}} \simeq \widetilde{\mathcal{R}}$, such that the following diagram is commutative (up to a natural isomorphism):

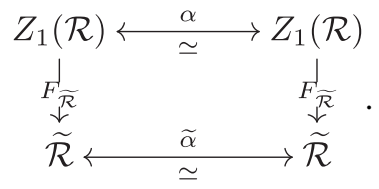

Remark 7. Note that $\widetilde{\alpha}$ in the above contains some redundant information. This can be seen from the fact that even when $\alpha=\mathrm{id}_{Z_{1}(\mathcal{R})}$, there can still be nontrivial $\widetilde{\alpha}$. We believe that such extra $\widetilde{\alpha}$ 's are higher structures (such as lower dimensional SPT or invertible phases) and should be regarded as equivalent when considering the classification of SPT/SET orders. See also Remark 10.

The above is just for SPT orders. In the following, we use a similar approach to develop a more general categorical theory to classify both SPT and SET orders. We also allow more general algebraic higher symmetry, by allowing $\mathcal{R}$ to be $\mathcal{V}$-local (recall Definition 28), to include at least both boson and fermion systems. When $\mathcal{V}=n \mathcal{V}$ ec, $\mathcal{R}$ describes the algebraic higher symmetry in bosonic systems. When $\mathcal{V}=n \mathrm{~s} \mathcal{V}$ ec, the fusion $n$-category of super vector spaces, $\mathcal{R}$ describes the algebraic higher symmetry in fermionic systems.

Remark 8. Physically, we think $\mathcal{V}$ as the building blocks of our system. $\mathcal{R}$ is built upon $\mathcal{V}$ with some additional symmetry that can be totally broken. $\beta: \mathcal{R} \rightarrow \mathcal{V}$ exactly describes the symmetry breaking that leaves only the intrinsic symmetry $\mathcal{V}$ of the building blocks which is not physically breakable (for example, fermion parity).

A gapped liquid state with symmetry $\mathcal{R}$ has excitations $\mathcal{C}$ that is equipped with a top-fully faithful monoidal functor $\iota: \mathcal{R} \rightarrow \mathcal{C}$. There is an anomaly-free topological order $\underline{\mathcal{C}}$ underlying $\mathcal{C}$ by breaking all the $\mathcal{R}$ symmetry. Mathematically, we may define $\underline{\mathcal{C}}$ to be the pushout (i.e., the colimit) of $\mathcal{V} \stackrel{\beta}{\leftarrow} \mathcal{R} \stackrel{\iota}{\rightarrow} \mathcal{C}$ in the category of fusion $n$-categories,

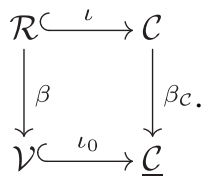

As a colimit, $\underline{\mathcal{C}}, \beta_{\mathcal{C}}$, and $\iota_{0}$ are uniquely determined by $\mathcal{V} \stackrel{\beta}{\leftarrow}$ $\mathcal{R} \stackrel{\iota}{\rightarrow} \mathcal{C}$ up to isomorphisms. In particular, for SPT orders, we take $\mathcal{C}=\mathcal{R}, \iota=\mathrm{id}_{\mathcal{R}}$, and then $\underline{\mathcal{C}}=\mathcal{V}, \beta_{\mathcal{C}}=\beta, \iota_{0}=\mathrm{id}_{\mathcal{V}}$ :

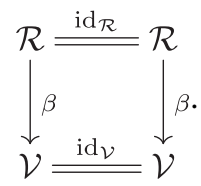

Alternatively, $\beta$ can be consider as condensing some excitations (which form an algebra $A_{\beta}$, and condensing means taking the modules over this algebra) in $\mathcal{R}$. Condensing the same excitations in $\mathcal{C}$ (identified via $\iota$ ), gives $\underline{\mathcal{C}}$.

$\underline{\mathcal{C}}$ constitutes a symmetry-breaking domain wall between $\underline{\mathcal{C}}$ and $\mathcal{C}$. Mathematically, $\underline{\mathcal{C}}$ is $\underline{\mathcal{C}}-\mathcal{C}$-bimodule; the left action is by fusion in $\underline{\mathcal{C}}$ and right action is by first mapping $\mathcal{C}$ into $\underline{\mathcal{C}}$ via $\beta_{\mathcal{C}}$ and then fusion in $\underline{\mathcal{C}}$. To emphasize this, we denote the bimodule by $\underline{\mathcal{C}}_{\beta_{\mathcal{C}}}$. The bulk of $\underline{\mathcal{C}}, \mathcal{C}$, as well as the domain wall $\underline{\mathcal{C}}_{\beta_{\mathcal{C}}}$, can be defined via bimodule functors (see Sec. III I): 


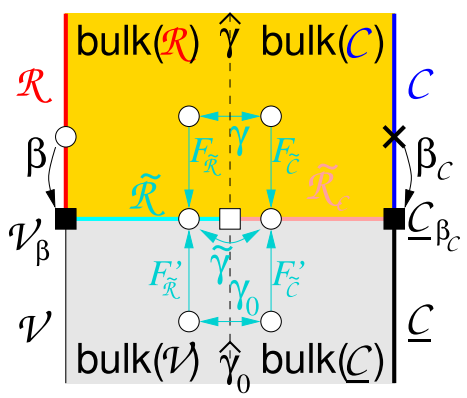

FIG. 24. $\mathcal{R}, \mathcal{V}$, and their domain wall $\mathcal{V}_{\beta}$ describe a trivial product state with symmetry $\mathcal{R}$ (as well as the symmetry-breaking $\mathcal{R} \stackrel{\beta}{\rightarrow} \mathcal{V}) . \mathcal{C}, \underline{\mathcal{C}}$, and their domain wall $\underline{\mathcal{C}}_{\beta_{\mathcal{C}}}$ describe a gapped liquid state with symmetry $\mathcal{R}$. The bulk of two structures (the categorical symmetry and its breaking) must coincide. This is just the anomaly matching condition, since the categorical symmetry can be viewed as anomaly. As a result, the $\mathcal{R}$-symmetric gapped liquid phase and the $\mathcal{R}$-symmetric trivial phase can be two phases of the same system. The data $\left(\mathcal{R} \stackrel{\beta}{\rightarrow} \mathcal{V}, \mathcal{R} \stackrel{\iota}{\hookrightarrow} \mathcal{C}, \hat{\gamma}, \tilde{\gamma}, \hat{\gamma}_{0}\right)$ classify the bosonic gapped liquid phases with $\mathcal{R}$-symmetry when $\mathcal{V}=n \mathcal{V}$ ec. They classify the fermionic gapped liquid phases with $\mathcal{R}$-symmetry when $\mathcal{V}=n$ s $\mathcal{V}$ ec.

(1) The bulk of $\underline{\mathcal{C}}$ is $Z_{1}(\underline{\mathcal{C}}):=\operatorname{Fun}_{\underline{\mathcal{C}} \mid \mathcal{\mathcal { C }}}(\underline{\mathcal{C}}, \underline{\mathcal{C}})$.

(2) The bulk of $\mathcal{C}$ is $Z_{1}(\mathcal{C}):=\operatorname{Fun}_{\mathcal{C} \mid \mathcal{C}}(\mathcal{C}, \mathcal{C})$.

(3) The bulk of the domain wall $\underline{\mathcal{C}}_{\beta_{\mathcal{C}}}$ is

$$
\widetilde{\mathcal{R}}_{\mathcal{C}}:=\operatorname{Fun}_{\underline{\mathcal{C}} \mathcal{C}}\left(\underline{\mathcal{C}}_{\beta_{\mathcal{C}}}, \underline{\mathcal{C}}_{\beta_{\mathcal{C}}}\right) \text {. }
$$

$\widetilde{\mathcal{R}}_{\mathcal{C}}$ is also a symmetry-breaking domain wall in the bulk, between $Z_{1}(\mathcal{C})$ and $Z_{1}(\mathcal{C})$.

(4) There are also bulk to wall functors

$$
\begin{aligned}
F_{\widetilde{\mathcal{R}}_{\mathcal{C}}}^{\prime}: Z_{1}(\underline{\mathcal{C}})=\operatorname{Fun}_{\underline{\mathcal{C}} \underline{\mathcal{C}}}(\underline{\mathcal{C}}, \underline{\mathcal{C}}) & \rightarrow \operatorname{Fun}_{\underline{\underline{C}} \mid \mathcal{C}}\left(\underline{\mathcal{C}}_{\beta_{\mathcal{C}}}, \underline{\mathcal{C}}_{\beta_{\mathcal{C}}}\right)=\widetilde{\mathcal{R}}_{\mathcal{C}} \\
f & \mapsto f\left(\mathbf{1}_{\underline{\mathcal{C}}}\right) \otimes-, \\
F_{\widetilde{\mathcal{R}}_{\mathcal{C}}}: Z_{1}(\mathcal{C})=\operatorname{Fun}_{\mathcal{C} \mid \mathcal{C}}(\mathcal{C}, \mathcal{C}) & \rightarrow \operatorname{Fun}_{\underline{\mathcal{C}} \mid \mathcal{C}}\left(\underline{\mathcal{C}}_{\beta_{\mathcal{C}}}, \underline{\mathcal{C}}_{\beta_{\mathcal{C}}}\right)=\widetilde{\mathcal{R}}_{\mathcal{C}} \\
f & \mapsto-\otimes \beta_{\mathcal{C}}\left(f\left(\mathbf{1}_{\mathcal{C}}\right)\right) .
\end{aligned}
$$

In conclusion, if we view $\underline{\mathcal{C}}, \mathcal{C}$ with a domain wall $\underline{\mathcal{C}}_{\beta_{\mathcal{C}}}$ between them as a whole, the bulk of this structure is given by $Z_{1}(\underline{\mathcal{C}}) \stackrel{F_{\widehat{\mathcal{R}}_{\mathcal{C}}}^{\prime}}{\longrightarrow} \widetilde{\mathcal{R}}_{\mathcal{C}} \stackrel{F_{\widetilde{R}_{\mathcal{C}}}}{\longleftarrow} Z_{1}(\mathcal{C})$ (see Fig. 24), i.e., two nondegenerate braided fusion $n$-categories $Z_{1}(\underline{\mathcal{C}})$ and $Z_{1}(\mathcal{C})$, a fusion $n$-category $\widetilde{\mathcal{R}}_{\mathcal{C}}$, and two bulk to wall functors $F_{\widetilde{\mathcal{R}}_{\mathcal{C}}}^{\prime}$ and $F_{\widetilde{\mathcal{R}}_{\mathcal{C}}}$. Physically, $Z_{1}(\underline{\mathcal{C}}) \stackrel{F_{\mathcal{R}_{\mathcal{C}}}^{\prime}}{\longrightarrow} \widetilde{\mathcal{R}}_{\mathcal{C}} \stackrel{F_{\widetilde{R}_{\mathcal{C}}}}{\longleftarrow} Z_{1}(\mathcal{C})$ is the categorical symmetry and how it breaks down corresponding to $\beta: \mathcal{R} \rightarrow \mathcal{V}$.

Clearly, the bulk of the product state with the $\mathcal{R}$-symmetry (and its breaking $\mathcal{R} \stackrel{\beta}{\rightarrow} \mathcal{V}$ ) is given by $Z_{1}(\mathcal{V}) \stackrel{F_{\widetilde{R}}^{\prime}}{\longrightarrow} \widetilde{\mathcal{R}} \stackrel{F_{\widetilde{\mathcal{R}}}}{\longleftarrow}$ $Z_{1}(\mathcal{R})$. As before, we require that the bulk of $\mathcal{C}$ coincide with the $\mathcal{R}$-symmetric product state, which amounts to say that there should be three equivalences $\gamma: Z_{1}(\mathcal{R}) \simeq$ $Z_{1}(\mathcal{C}), \widetilde{\gamma}: \widetilde{\mathcal{R}} \simeq \widetilde{\mathcal{R}}_{\mathcal{C}}$, and $\gamma_{0}: Z_{1}(\mathcal{V}) \simeq Z_{1}(\underline{\mathcal{C}})$. The criteria for these equivalences to preserve $\mathcal{R}$ are given below:

Proposition 36. An anomaly-free gapped liquid phase in $n$-dimensional space with a generalized anomaly-free algebraic higher symmetry described by a $\mathcal{V}$-local $\mathcal{R}$ is characterized by the data $\iota: \mathcal{R} \hookrightarrow \mathcal{C}$, and $\hat{\gamma}, \tilde{\gamma}, \hat{\gamma}_{0}$, where $\mathcal{C}$ is a fusion $n$-category, $\hat{\gamma}$ is an invertible domain wall between $\operatorname{bulk}(\mathcal{R})$ and $\operatorname{bulk}(\mathcal{C}), \hat{\gamma}_{0}$ is an invertible domain wall between bulk $(\mathcal{V})$ and bulk $(\underline{\mathcal{C}})$, and $\tilde{\gamma}: \widetilde{\mathcal{R}} \simeq \widetilde{\mathcal{R}}_{\mathcal{C}}$ is a monoidal equivalence. The two invertible domain walls induce braided equivalences $\gamma: Z_{1}(\mathcal{R}) \simeq Z_{1}(\mathcal{C})$ and $\gamma_{0}: Z_{1}(\mathcal{V}) \simeq Z_{1}(\underline{\mathcal{C}})$. The three equivalences $\gamma, \widetilde{\gamma}, \gamma_{0}$ must render the following diagram commutative:

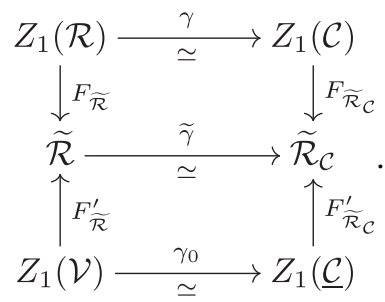

In the above, we have used the invertible domain walls $\hat{\gamma}$ and $\hat{\gamma}_{0}$ to capture invertible topological orders. We use the equivalences $\gamma$ and $\gamma_{0}$ of braided fusion higher categories, induced by the invertible domain walls, to formulate the condition to select the proper domain walls.

In particular, taking $\mathcal{C}=\mathcal{R}$ and then $\underline{\mathcal{C}}=\mathcal{V}, \widetilde{\mathcal{R}}_{\mathcal{C}}=\widetilde{\mathcal{R}}$, we obtain a classification of $\mathcal{R}$-SPT orders:

Proposition 37. An SPT phase in $n$-dimensional space with a generalized anomaly-free algebraic higher symmetry described by a $\mathcal{V}$-local $\mathcal{R}$ is characterized by the three automorphisms $\alpha: Z_{1}(\mathcal{R}) \simeq Z_{1}(\mathcal{R}), \widetilde{\alpha}: \widetilde{\mathcal{R}} \simeq \widetilde{\mathcal{R}}, \alpha_{0}: Z_{1}(\mathcal{V}) \simeq$ $Z_{1}(\mathcal{V})$, rendering the following diagram commutative:

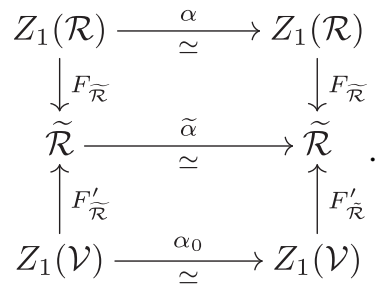

The above triples of automorphisms $\left(\alpha, \widetilde{\alpha}, \alpha_{0}\right)$ that label different $\mathcal{R}$-SPT orders can be composed, which correspond to the stacking of the SPT orders.

If we choose $\mathcal{V}=n \mathcal{V}$ ec, the above classifies SET/SPT orders for bosonic systems with an algebraic higher symmetry. If we choose $\mathcal{V}=n \mathrm{~s} \mathcal{V}$ ec, the above classifies SET $/$ SPT orders for fermionic systems with a generalized algebraic higher symmetry. Again, as pointed out in Remarks 7 and 10, it is very likely that different choices $\tilde{\gamma}, \gamma_{0}$ or $\widetilde{\alpha}, \alpha_{0}$ correspond to the same SET/SPT order, and thus only $\gamma$ or $\alpha$ needs to be kept.

In this formulation, there is no need to assume that $\mathcal{R}$ is symmetric or even braided. But assuming $\mathcal{R}, \mathcal{V}$ and $\beta: \mathcal{R} \rightarrow$ $\mathcal{V}$ are braided, we want to show that the Proposition 36 and Proposition 33 are equivalent. We sketch a tentative proof here. There is a canonical braided embedding $\iota_{\mathcal{R}}: \mathcal{R} \hookrightarrow$ $Z_{1}(\mathcal{R})$. Then consider the pushout of $\mathcal{V} \stackrel{\beta}{\leftarrow} \mathcal{R} \stackrel{\mathfrak{L}_{\mathcal{R}}}{\longrightarrow} Z_{1}(\mathcal{R})$. In the category of fusion $n$-categories, the pushout is just $\widetilde{\mathcal{R}}$ :

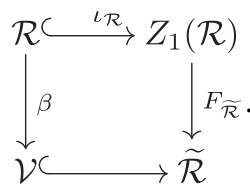


Indeed, $\beta$ can be considered as condensing some excitations $A_{\beta}$ in $\mathcal{R}$. Condensing the same excitations in $Z_{1}(\mathcal{R})$ (identified via $\underset{\mathcal{R}}{\iota_{\mathcal{R}}}$ ) gives $\widetilde{\mathcal{R}}$. Moreover, $Z_{1}(\mathcal{V})$ should be a full subcategory of $\widetilde{\mathcal{R}}$ corresponding to the deconfined excitations and $F_{\widetilde{\mathcal{R}}}^{\prime}$ is the embedding. Therefore, the embedding $\iota_{\mathcal{R}}$ determines all the other structures $Z_{1}(\mathcal{V}), \widetilde{\mathcal{R}}, F_{\widetilde{\mathcal{R}}}, F_{\widetilde{\mathcal{R}}}^{\prime}$. Also $\gamma: Z_{1}(\mathcal{R}) \simeq Z_{1}(\mathcal{C})$ with such embedding $\iota_{\mathcal{R}}$ determines $\tilde{\gamma}$ and $\gamma_{0}$. Then it should be straightforward to verify that (155) is equivalent to (147).

Example 3. For $\mathcal{R}=\mathcal{C}=\mathcal{R e p} G, \mathcal{V}=\mathcal{V e c}, \beta: \mathcal{R e p} G \rightarrow$ $\mathcal{V}$ ec, the forgetful functor, we have $\widetilde{\mathcal{R}}=\mathcal{V}_{\mathrm{ec}}$. Since $F_{\widetilde{\mathcal{R}}}$ corresponds to condensing the algebra $\operatorname{Fun}(G) \in \mathcal{R} \operatorname{ep} G$, preserving the embedding $\mathcal{R e p} G \hookrightarrow Z_{1}(\mathcal{R e p} G)$ is the same as preserving $\operatorname{Fun}(G)$ and thus $\tilde{F}_{\mathcal{R}}$. The $G=\mathbb{Z}_{2} \times \mathbb{Z}_{2}$ case is explicitly calculated in Sec. VII C 7.

\section{Second version of classification based on condensable algebra}

In this section, we are going to describe another version of classification. Let us first consider an $\mathcal{R}$-SPT state characterized by a pair $(\mathcal{R}, \alpha)$, where $\alpha$ is a braided automorphism of $Z_{1}(\mathcal{R})$ (see Fig. 19). We would like to explore other equivalent ways to select proper $\alpha$ 's. We first restrict to bosonic systems for simplicity, assuming that $\mathcal{R}$ is a local fusion $n$-category. A key feature of SPT order is that a SPT state has no topological order; i.e., it becomes a product state if we break the symmetry. How to impose such a condition, when we use the holographic point view of the symmetry $\mathcal{R}$ ?

Here we would like to point out that if we stack $\mathcal{R}$ and its dual $\widetilde{\mathcal{R}}$ through their common bulk $\mathcal{M}=Z_{1}(\mathcal{R})$, denoted as $\mathcal{R} \otimes \widetilde{\mathcal{R}}^{\text {rev }}$, we get a trivial product state $n \mathcal{V}$ ec (see Fig. 6). We may use this property to define a more general notion of dual symmetry.

Definition 30. Let $\mathcal{M}$ be the braided fusion $n$-category describing excitations in a $(n+1) \mathrm{d}$ anomaly-free bulk topological order, and $\mathcal{R}$ and $\mathcal{B}$ (together with bulk to boundary functors $\mathcal{M} \rightarrow \mathcal{R}, \mathcal{M} \rightarrow \mathcal{B}$ ) be two $n$ d boundaries of $\mathcal{M}$. $\mathcal{R}$ and $\mathcal{B}$ are said to be dual to each other if

$$
\mathcal{R} \underset{\mathcal{M}}{\otimes} \mathcal{B}^{\text {rev }}=n \mathcal{V e c}
$$

In fact, there is an one-to-one correspondence between dual symmetries of $\mathcal{R}$ and the monoidal functors $\mathcal{R} \rightarrow n \mathcal{V e c}$. If a boundary $\mathcal{B}$ of $Z_{1}(\mathcal{R})$ satisfies $\mathcal{R} \underset{Z_{1}(\mathcal{R})}{\otimes} \mathcal{B}^{\text {rev }}=n \mathcal{V}$ ec, we may define

$$
\begin{aligned}
\beta_{\mathcal{B}}: \mathcal{R} & \rightarrow n \mathcal{V e c}=\mathcal{R} \underset{Z_{1}(\mathcal{R})}{\otimes} \mathcal{B}^{\text {rev }}, \\
x & \mapsto x \underset{Z_{1}(\mathcal{R})}{\otimes} \mathbf{1}_{\mathcal{B}} .
\end{aligned}
$$

Recall that for the given $\beta: \mathcal{R} \rightarrow n \mathcal{V}$ ec, the dual symmetry is defined by $\widetilde{\mathcal{R}}=\operatorname{Fun}_{\mathcal{R} \mid n \mathcal{V e c}}\left(n \mathcal{V} \mathrm{ec}_{\beta}, n \mathcal{V} \mathrm{Uec}_{\beta}\right)$ [see Fig. 23(a)]. We have the following correspondence:

Proposition 38. The maps $\mathcal{B} \mapsto \beta_{\mathcal{B}} \quad$ and $\quad \beta \mapsto$ $\operatorname{Fun}_{\mathcal{R} \mid n \mathcal{V e c}}\left(n \mathcal{V} \mathrm{ec}_{\beta}, n \mathcal{V} \mathrm{ec}_{\beta}\right)$ are inverse to each other.

In particular, $\mathcal{R}$ also defines a monoidal functor $\mathcal{B} \rightarrow$ $n \mathcal{V e c}$.

Proposition 39. if $Z_{1}(\mathcal{R})=Z_{1}(\mathcal{B})$ and $\mathcal{R} \underset{Z_{1}(\mathcal{R})}{\otimes} \mathcal{B}^{\text {rev }}=$ $n \mathcal{V e c}$, then both $\mathcal{R}$ and $\mathcal{B}$ are local fusion $n$-categories.

Physically, when $\mathcal{R}$ and $\widetilde{\mathcal{R}}$ are dual to each other, every excitation in $\mathcal{M}=Z_{1}(\mathcal{R})$, either condenses to $\mathcal{R}$-boundary or

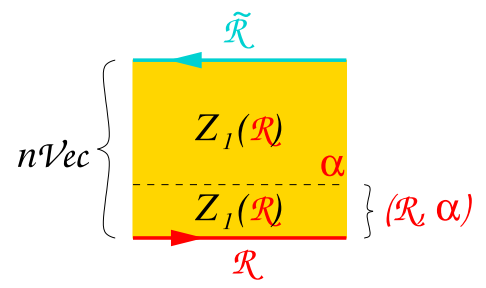

FIG. 25. $(\mathcal{R}, \alpha) \equiv \mathcal{R} \underset{Z_{1}(\mathcal{R})}{\otimes} \alpha \underset{Z_{1}(\mathcal{R})}{\otimes}$ describes an SPT state only if it can be canceled by $\widetilde{\mathcal{R}}$ (i.e., producing a product state $n \mathcal{V}$ ec).

condenses to $\widetilde{\mathcal{R}}$-boundary, or both. In this case, every excitation in $\mathcal{M}$ is condensed and the resulting state is trivial. We know that $\mathcal{R}$ can be obtained from $\mathcal{M}$ via a Lagrangian condensable algebra $A_{\mathcal{R}}$ in $\mathcal{M}$. Similarly, $\widetilde{\mathcal{R}}$ can be obtained from $\mathcal{M}$ via another Lagrangian condensable algebra $A_{\widetilde{\mathcal{R}}}$. Roughly, a condensable algebra is formed by excitations with trivial mutual statistics with each other, and those excitations can all condense simultaneously to form a gapped boundary (see Fig. 6). Thus, in the $\mathcal{R}$ boundary, the excitations in $A_{\mathcal{R}}$ condense. The noncondensing excitations become the boundary excitations that is described by $\mathcal{R}$. So, roughly, $\mathcal{R}=\mathcal{M} / A_{\mathcal{R}}$. (In precise mathematical language, $\mathcal{R}$ identifies with the category of modules over $A_{\mathcal{R}}$ in $\mathcal{M}$.) Similarly, $\widetilde{\mathcal{R}}=\mathcal{M} / A_{\widetilde{\mathcal{R}}}$. When $\mathcal{R}$ and $\widetilde{\mathcal{R}}$ are dual to each other, the overlap of $A_{\mathcal{R}}$ and $A_{\widetilde{\mathcal{R}}}$ is minimal and is given by the trivial excitations. Also $A_{\mathcal{R}}$ and $A_{\tilde{\mathcal{R}}}$ together generate the whole $\mathcal{M}$. (More precisely, any excitation in $\mathcal{M}$ is contained in $A_{\mathcal{R}} \otimes A_{\tilde{\mathcal{R}}}$.) Thus, roughly, $A_{\mathcal{R}} \otimes A_{\widetilde{\mathcal{R}}} \sim \mathcal{M}$. We see that $A_{\mathcal{R}}$ is formed by excitations in $\widetilde{\mathcal{R}}$ and $A_{\widetilde{\mathcal{R}}}$ is formed by excitations in $\mathcal{R}$.

Proposition 38 tells us that dual symmetry is an equivalent way to describe symmetry breaking. If $\mathcal{R}$ can be canceled by its dual $\mathcal{R} \underset{\mathcal{M}}{\otimes} \widetilde{\mathcal{R}}^{\text {rev }}=n \mathcal{V}$ ec, then $\mathcal{R}$ (as well as $\widetilde{\mathcal{R}}$ ) is a local fusion $n$-category; i.e., $\mathcal{R}$ can be reduced to the trivial product state if we break the symmetry: $\mathcal{R} \stackrel{\beta}{\rightarrow} n \mathcal{V}$ ec. Since $\mathcal{R}$ can be viewed as $(\mathcal{R}, \alpha=$ id $)$ in Fig. 19 , we see that $(\mathcal{R}$, id $)$ can be canceled by $\widetilde{\mathcal{R}}$, which implies that $(\mathcal{R}$, id $)$ is a product state if we break the symmetry. This implies that if we do not break the symmetry, then $(\mathcal{R}$, id) is a SPT state. Therefore, to see if $(\mathcal{R}, \alpha)$ is a SPT state or not, we can just check if it can be canceled by $\widetilde{\mathcal{R}}$ or not. This allows us to obtain (see Fig. 25) the following:

Proposition 40. $(\mathcal{R}, \alpha) \equiv \mathcal{R} \underset{Z_{1}(\mathcal{R})}{\otimes} \alpha \underset{Z_{1}(\mathcal{R})}{\otimes}$ describes a bosonic $\mathcal{R}$-SPT state if the automorphism $\alpha$ of $Z_{1}(\mathcal{R})$ satisfies

$$
\mathcal{R} \underset{Z_{1}(\mathcal{R})}{\otimes} \alpha \underset{Z_{1}(\mathcal{R})}{\otimes} \widetilde{\mathcal{R}}^{\text {rev }} \simeq n \mathcal{V e c}
$$

Using the condensable algebra, we find that one class of the solutions of (160) is given by $\alpha$ 's that keep the $A_{\mathcal{R}}$ unchanged (see Fig. 26),

$$
\alpha\left(A_{\mathcal{R}}\right) \stackrel{\mu}{\simeq} A_{\mathcal{R}},
$$

where $\mu$ is an algebra isomorphism. In this case, Fig. 26(a) can be deformed into Fig. 26(b). By comparing Fig. 26(b) with Fig. 25, we see that both $\mathcal{R} \underset{Z_{1}(\mathcal{R})}{\otimes} \alpha \underset{Z_{1}(\mathcal{R})}{\otimes}$ and $\mathcal{R} \underset{Z_{1}(\mathcal{R})}{\otimes}$ are determined by the same condensable algebra $A_{\mathcal{R}}$, and hence are equivalent: $\mathcal{R} \underset{Z_{1}(\mathcal{R})}{\otimes} \alpha \underset{Z_{1}(\mathcal{R})}{\otimes} \simeq \mathcal{R} \underset{Z_{1}(\mathcal{R})}{\otimes}$. This allows us to 


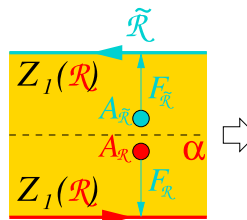

(a) $\mathcal{R}$

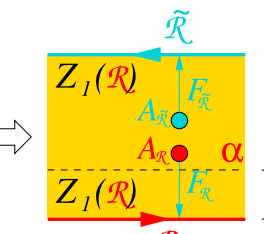

(b) $R$
$(\mathcal{R} \alpha)$

FIG. 26. If $\alpha$ keeps the $A_{\mathcal{R}}$ unchanged, then $(\mathcal{R}, \alpha) \equiv \mathcal{R} \underset{Z_{1}(\mathcal{R})}{\otimes}$ $\alpha \underset{Z_{1}(\mathcal{R})}{\otimes}$ is also determined by the condensable algebra $A_{\mathcal{R}}$ and is equivalent to $\mathcal{R} \underset{Z_{1}(\mathcal{R})}{\otimes}$.

show that

$$
\mathcal{R} \underset{Z_{1}(\mathcal{R})}{\otimes} \alpha \underset{Z_{1}(\mathcal{R})}{\otimes} \widetilde{\mathcal{R}}^{\text {rev }} \simeq \mathcal{R} \underset{Z_{1}(\mathcal{R})}{\otimes} \widetilde{\mathcal{R}}^{\text {rev }} \simeq n \mathcal{V e c}
$$

$\mathcal{R} \underset{Z_{1}(\mathcal{R})}{\otimes} \alpha \underset{Z_{1}(\mathcal{R})}{\otimes} \simeq \mathcal{R} \underset{Z_{1}(\mathcal{R})}{\otimes}$ also tell us that the SPT state described by $\mathcal{R} \otimes \alpha \underset{\otimes}{\otimes}$ is equivalent to the SPT state described by $\mathcal{R} \otimes$, which is the trivial SPT state. In fact, $\alpha$ that keeps $A_{\mathcal{R}} \sim \stackrel{\mathcal{R}}{Z_{1}(\mathcal{R})}$ unchanged may change $\mathcal{R}$. Thus, we believe that $\mathcal{R} \underset{Z_{1}(\mathcal{R})}{\otimes} \alpha \underset{Z_{1}(\mathcal{R})}{\otimes}$ and $\mathcal{R} \underset{Z_{1}(\mathcal{R})}{\otimes}$ only differ by a relabeling of the symmetry (i.e., differ by an automorphism of $\mathcal{R}$ ), and thus correspond to the same SPT state.

Equation (160) has another class of solutions, given by $\alpha$ 's that keep $A_{\widetilde{\mathcal{R}}}$ unchanged:

$$
\alpha\left(A_{\widetilde{\mathcal{R}}}\right) \stackrel{\mu}{\simeq} A_{\widetilde{\mathcal{R}}}
$$

By comparing Fig. 27(b) with Fig. 25, we see that $\underset{Z_{1}(\mathcal{R})}{\otimes} \alpha \underset{Z_{1}(\mathcal{R})}{\otimes}$ $\widetilde{\mathcal{R}}^{\text {rev }} \simeq \underset{Z_{1}(\mathcal{R})}{\otimes} \widetilde{\mathcal{R}}^{\text {rev }}$. This allows us to show that, indeed,

$$
\mathcal{R} \underset{Z_{1}(\mathcal{R})}{\otimes} \alpha \underset{Z_{1}(\mathcal{R})}{\otimes} \widetilde{\mathcal{R}}^{\text {rev }} \simeq \mathcal{R} \underset{Z_{1}(\mathcal{R})}{\otimes} \widetilde{\mathcal{R}}^{\text {rev }} \simeq n \mathcal{V e c} .
$$

As we have mentioned, the condensable algebra $A_{\widetilde{\mathcal{R}}}$ is formed by excitations in $\mathcal{R}$. So, keeping $A_{\tilde{\mathcal{R}}}$ part of $\mathcal{M}$ unchanged corresponds to keeping $\mathcal{R}$ part of $\mathcal{M}$ unchanged. Therefore, the automorphisms $\alpha$, that satisfy (163), do not change the $\underset{\mathcal{R}}{\mathcal{R}}$

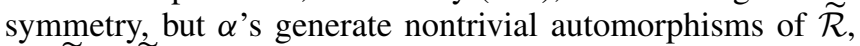
$\widetilde{\alpha}: \widetilde{\mathcal{R}} \simeq \widetilde{\mathcal{R}}$ (see Fig. 28).

Figure 28 also describes the canonical symmetry-breaking boundary, $n \mathcal{V} \operatorname{ec}_{\beta} \underset{\widetilde{\mathcal{R}}}{\otimes} \underset{\widetilde{\alpha}}{\otimes}$, of the SPT state $\mathcal{R} \underset{Z_{1}(\mathcal{R})}{\otimes} \alpha \underset{Z_{1}(\mathcal{R})}{\otimes}$. We see that different pairs of automorphisms $(\alpha, \widetilde{\alpha})$ give rise to different boundaries (due to different $\widetilde{\alpha}$ 's). This leads to the following result:

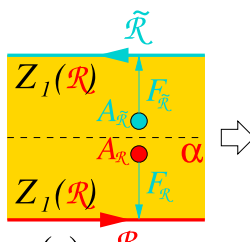

(a) $R$

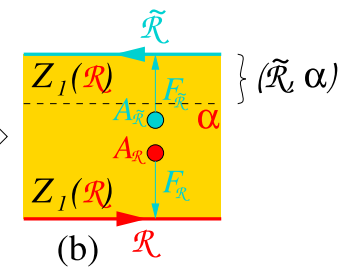

(b) $R$
FIG. 27. If $\alpha$ keeps the $A_{\widetilde{\mathcal{R}}}$ unchanged, then $(\widetilde{\mathcal{R}}, \alpha) \equiv$ $\underset{Z_{1}(\mathcal{R})}{\otimes} \alpha \underset{Z_{1}(\mathcal{R})}{\otimes} \widetilde{\mathcal{R}}^{\text {rev }} \simeq \underset{Z_{1}(\mathcal{R})}{\otimes} \widetilde{\mathcal{R}}^{\text {rev }}$

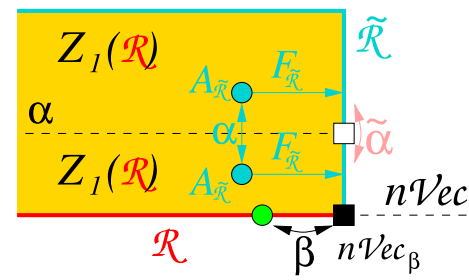

FIG. 28. The $\mathcal{R}$-SPT orders are classified by the automorphisms $\alpha$ of $Z_{1}(\mathcal{R})$ that keep $A_{\tilde{\mathcal{R}}}$ unchanged. $\alpha$ corresponds to an invertible domain wall in the bulk (the dash-line), which also has an invertible boundary (the white square). The boundary-bulk relation between $Z_{1}(\mathcal{R})$ and $\widetilde{\mathcal{R}}$ is described by the bulk-to-boundary functor $F_{\widetilde{\mathcal{R}}}$, which maps the condensable algebra $A_{\widetilde{\mathcal{R}}}$ to the trivial excitons on the $\widetilde{\mathcal{R}}$ boundary.

Proposition 41. Bosonic SPT phases in $n$-dimensional space with an anomaly-free algebraic higher symmetry $\mathcal{R}$ are classified (up to invertible topological orders) by braided equivalences $\alpha: Z_{1}(\mathcal{R}) \simeq Z_{1}(\mathcal{R})$ together with algebra isomorphisms $\mu: \alpha\left(A_{\tilde{\mathcal{R}}}\right) \simeq A_{\tilde{\mathcal{R}}}$.

We would like to remark that, for a given $\alpha$, different choices of $\mu$ differ by automorphisms of the condensable algebra $A_{\widetilde{\mathcal{R}}}$. Those different $\mu$ 's may lead to the same SPT order. This is because if we gauge the $\mathcal{R}$-symmetry in a SPT state, the resulting topological order does not depend on $\mu$ (see Remark 10). Thus, the bosonic SPT phases with a $\mathcal{R}$ symmetry may actually be classified by $\alpha$ 's rather than the pairs $(\alpha, \mu)$ 's.

We can generalize the above result to include SET orders with $\mathcal{R}$-symmetry for bosonic or fermionic systems, as well as invertible topological orders by using invertible domain walls (see Fig. 29):

Proposition 42. Anomaly-free gapped liquid phases in $n$-dimensional space with a generalized anomaly-free algebraic higher symmetry $\mathcal{R}$ are classified by the data $(\mathcal{R} \stackrel{\iota}{\hookrightarrow}$ $\mathcal{C}, \hat{\gamma}, \mu)$, where $\mathcal{R}$ is a $\mathcal{V}$-local fusion $n$-category, $\mathcal{C}$ is a fusion $n$-category that includes $\mathcal{R}, \hat{\gamma}$ is a invertible domain wall between $\operatorname{bulk}(\mathcal{R})$ and $\operatorname{bulk}(\mathcal{C})$, and $\mu: \gamma\left(A_{\widetilde{\mathcal{R}}}\right) \simeq A_{\widetilde{\mathcal{R}}_{\mathcal{C}}}$ is an algebra isomorphism. Here $\gamma: Z_{1}(\mathcal{R}) \simeq Z_{1}(\mathcal{C})$ is a braided equivalence, $\widetilde{\mathcal{R}}_{\mathcal{C}}$ is defined in Proposition 36 , and $A_{\widetilde{\mathcal{R}}}, A_{\widetilde{\mathcal{R}}_{\mathcal{C}}}$ are the condensable algebras in $Z_{1}(\mathcal{R}), Z_{1}(\mathcal{C})$ that produces the

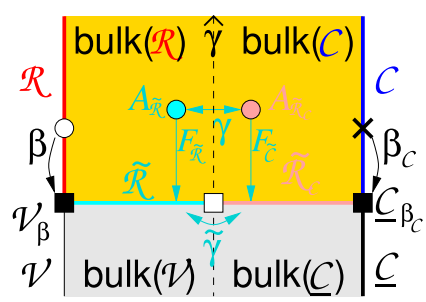

FIG. 29. Similar to Fig. 24, but here the invertible domain wall $\hat{\gamma}$ induces an automorphism $\gamma$, which is required to map the two condensable algebras, $A_{\mathcal{R}}$ and $A_{\tilde{\mathcal{R}}_{\mathcal{C}}}$, into each other: $\mu: \gamma\left(A_{\tilde{\mathcal{R}}}\right) \simeq$ $A_{\tilde{\mathcal{R}}_{\mathcal{C}}}$. The data $(\mathcal{R} \stackrel{\beta}{\rightarrow} \mathcal{V}, \mathcal{R} \stackrel{\iota}{\hookrightarrow} \mathcal{C}, \hat{\gamma}, \mu)$ classify the bosonic gapped liquid phases with $\mathcal{R}$-symmetry when $\mathcal{V}=n \mathcal{V}$ ec. They classify the fermionic gapped liquid phases with $\mathcal{R}$-symmetry when $\mathcal{V}=n \mathrm{~s} \mathcal{V}$ ec. 
$\widetilde{\mathcal{R}}, \widetilde{\mathcal{R}}_{\mathcal{C}}$ domain walls between $Z_{1}(\mathcal{R}), Z_{1}(\mathcal{C})$ and $Z_{1}(\mathcal{V}), Z_{1}(\underline{\mathcal{C}})$, respectively.

Although we have included $\mu$ in the above, it is possible that different choices of $\mu$ correspond to the same gapped liquid phases, as we discussed later in Remark 10. Thus, the different gapped liquid phases may actually be classified by the data $(\mathcal{R} \stackrel{\iota}{\hookrightarrow} \mathcal{C}, \hat{\gamma})$ such that $\gamma\left(A_{\widetilde{\mathcal{R}}}\right) \simeq A_{\widetilde{\mathcal{R}}_{\mathcal{C}}}$.

When $\mathcal{V}=n \mathcal{V}$ ec, the above classifies SET/SPT orders in bosonic systems. When $\mathcal{V}=n \mathrm{~s} \mathcal{V e c}$, the above classifies SET/SPT orders in fermionic systems.

Remark 9. Here we would like to sketch the reasoning that Proposition 36 and Proposition 42 are equivalent. From the condensation point of view, condensing the algebra $A_{\beta}$ in $\mathcal{R}$ induces the symmetry breaking $\beta: \mathcal{R} \rightarrow \mathcal{V}$. Similarly, the symmetry breaking $F_{\widetilde{\mathcal{R}}}: Z_{1}(\mathcal{R}) \rightarrow \widetilde{\mathcal{R}}$ in the bulk is induced by condensing the algebra $A_{\widetilde{\mathcal{R}}}$ in $Z_{1}(\mathcal{R}) . A_{\widetilde{\mathcal{R}}}$ is the lift of $A_{\beta}$ in the bulk, i.e., $A_{\beta}=F_{\mathcal{R}}\left(A_{\widetilde{\mathcal{R}}}\right)$, where $F_{\mathcal{R}}: Z_{1}(\mathcal{R}) \rightarrow \mathcal{R}$ is the forgetful functor. Intuitively, we can think that $A_{\widetilde{\mathcal{R}}}$ replaces the role of embedding $\mathcal{R} \hookrightarrow Z_{1}(\mathcal{R})$; instead of embedding $\mathcal{R}$ into the bulk which is only possible when $\mathcal{R}$ is braided, we lift the algebra $A_{\beta}$ in $\mathcal{R}$ to the algebra $A_{\widetilde{\mathcal{R}}}$ in $Z_{1}(\mathcal{R}) . A_{\beta}$ consists of all objects in $\mathcal{R}$ when $\mathcal{V}=n \mathcal{V}$ ec. Mathematically, $\widetilde{\mathcal{R}}$ should be the category of modules over $A_{\widetilde{\mathcal{R}}}$ in $Z_{1}(\mathcal{R})$ while $Z_{1}(\mathcal{V})$ should be the full subcategory of local modules, with $F_{\tilde{\mathcal{R}}}^{\prime}$ being the embedding (see Fig. 24). (Physically, $Z_{1}(\mathcal{V})$ corresponds the deconfined excitations after condensation while $\widetilde{\mathcal{R}}$ includes both confined and deconfined excitations.) Therefore, $\gamma$ together with $\mu: \gamma\left(A_{\widetilde{\mathcal{R}}}\right) \simeq A_{\widetilde{\mathcal{R}}_{\mathcal{C}}}$ determines $\tilde{\gamma}$ as an equivalence functor between the categories of modules over $A_{\widetilde{\mathcal{R}}}$ and $A_{\widetilde{\mathcal{R}}_{\mathcal{C}}}$; $\gamma_{0}$ is the restriction of $\tilde{\gamma}$ to $Z_{1}(\mathcal{V})$. Equation (155) is equivalent to saying that $\gamma$ preserves the lifted algebra $\gamma\left(A_{\widetilde{\mathcal{R}}}\right) \stackrel{\mu}{\simeq} A_{\widetilde{\mathcal{R}}_{\mathcal{C}}}$.

\section{5. $\mathcal{R}$-gauge theory obtained by "gauging" the algebraic higher symmetry $\mathcal{R}$}

Using the data $(\mathcal{C}, \iota: \mathcal{R} \hookrightarrow \mathcal{C}, \hat{\gamma}: \operatorname{bulk}(\mathcal{R}) \simeq \operatorname{bulk}(\mathcal{C}))$, we can explicitly construct the corresponding gapped liquid state with anomaly-free symmetry $\mathcal{R}$ that the data describe. This is done in Fig. 18. Since the gapped liquid state has the symmetry $\mathcal{R}$, we can gauge the symmetry $\mathcal{R}$ to obtain a new topologically ordered stated with no symmetry. This is achieved in Fig. 30(a), by stacking $\mathcal{R}$ and $\mathcal{C}$ through $\operatorname{bulk}(\mathcal{R}) \stackrel{\hat{\mathcal{\Sigma}}}{\simeq}$ bulk $(\mathcal{C})$, with an invertible domain wall $\hat{\gamma}$ in the middle. We denote such a stacking by $\mathcal{R} \otimes \hat{\gamma} \otimes \mathcal{C}^{\text {rev }}$. The resulting topologically ordered state is anomaly-free since it is surrounded by the trivial product state (with its codimension- 2 excitations described by $n \mathcal{V e c}$ ). As a bonus, such a gauging picture leads to third version of classification, which will be described in the next subsection.

Both 0-symmetries and higher symmetries have an holonomy interpretation, which allows us to gauge them via a geometric approach. In contrast, the above proposal to "gauge" algebraic higher symmetries (which include 0symmetries and higher symmetries) is a purely algebraic approach. No geometric interpretation is used.

To understand such a proposal, let us consider a very simple case, by assuming $\hat{\gamma}=$ trivial, $n=2$, and $\mathcal{C}=\mathcal{R}=$ $2 \mathcal{R e p} G$. So the boundary $\mathcal{R}$ is in $2 \mathrm{~d}$ while the bulk $\operatorname{bulk}(\mathcal{R})$ is

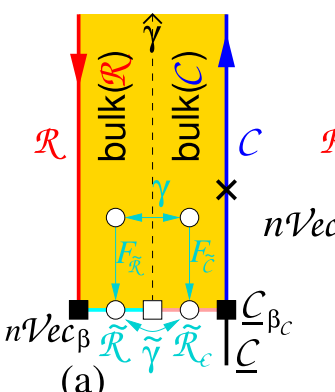

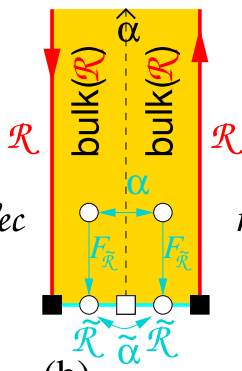

(b)

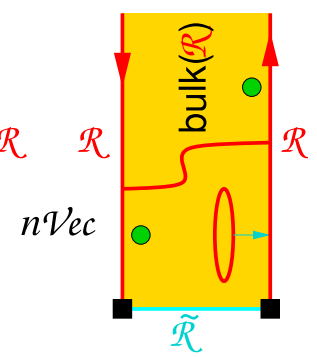

(c)
FIG. 30. (a) Same as Fig. 24, assuming $\mathcal{V}=n \mathcal{V}$ ec. The stacking of the two boundaries $\mathcal{R}$ and $\mathcal{C}$ through the bulk bulk $(\mathcal{R}) \stackrel{\hat{\mathcal{V}}}{\simeq} \operatorname{bulk}(\mathcal{C})$ with an invertible domain wall $\hat{\gamma}$ (denoted by $\mathcal{R} \underset{\text { bulk(R) }}{\otimes} \hat{\gamma} \underset{\text { bulk }(\mathcal{C})}{\otimes} \mathcal{C}^{\text {rev }}$ ) gives rise to an anomaly-free topological order with no symmetry, which is obtained by gauging the algebraic higher symmetry $\mathcal{R}$ in the gapped liquid state (SET or SPT state) characterized by the data $(\mathcal{C}, \iota: \mathcal{R} \hookrightarrow \mathcal{C}, \hat{\gamma}: \operatorname{bulk}(\mathcal{R}) \simeq \operatorname{bulk}(\mathcal{C}))$. (b) When $\mathcal{C}=\mathcal{R}$ and $\hat{\gamma}=\hat{\alpha}, \mathcal{R} \underset{\operatorname{lol}}{\otimes} \hat{\alpha} \otimes \mathcal{R}^{\text {rev }}$ describes the twisted $\mathcal{R}$-gauge theory $\mathrm{GT}_{\mathcal{R}, \alpha}^{n+1}$ obtained by gauging the symmetry $\mathcal{R}$ in the SPT state stacked with tirivial or nontrivial invertible topological orders. (c) When $\mathcal{C}=\mathcal{R}$ and $\hat{\gamma}=$ trivial, $\mathcal{R} \underset{\operatorname{bulk}(\mathcal{R})}{\otimes} \mathcal{R}^{\text {rev }}$ describes the $\mathcal{R}$ gauge theory $\mathrm{GT}_{\mathcal{R}}^{n+1}$ obtained from a symmetric product state by gauging its symmetry $\mathcal{R}$.

in 3 d. The resulting state $\mathcal{R} \otimes \mathcal{R}^{\text {rev }}$ given by Fig. 30(c) is actually a $2 \mathrm{~d}$ gauge theory with group $G$ (i.e., the $2 \mathrm{~d}$
topological order $\mathrm{GT}_{G}^{3}$ ). To see this, we note that the bulk $\operatorname{bulk}(\mathcal{R})$ is the $3 \mathrm{~d}$ gauge theory with group $G$ (i.e., the $3 \mathrm{~d}$ topological order $\left.\mathrm{GT}_{G}^{4}=\operatorname{bulk}(2 \mathcal{R} \operatorname{ep} G)\right]$. The $2 \mathrm{~d}$ boundary $\mathcal{R}=2 \mathcal{R} \operatorname{ep} G$ is obtained from the $3 \mathrm{~d} G$-gauge theory $\mathrm{GT}_{G}^{4}$ by condensing the $G$-flux loops. Thus, a $G$-flux loop in the bulk corresponds to a trivial excitation in the $2 \mathrm{~d} G$-gauge theory order $\mathrm{GT}_{G}^{3}$. A $G$-flux string connecting two boundaries corresponds to a point-like $G$-flux excitation in the 2 d $G$-gauge theory $\mathrm{GT}_{G}^{3}$. The point-like $G$-charges in the $3 \mathrm{~d} G$-gauge theory $\mathrm{GT}_{G}^{4}$ becomes the point-like $G$-charges in the $2 \mathrm{~d} G$-gauge theory $\mathrm{GT}_{G}^{3}$. This suggests that, in general, $\mathcal{R} \underset{\text { bulk( } \mathcal{R})}{\otimes} \mathcal{R}^{\text {rev }}$ in Fig. 30(c) is a $\mathcal{R}$-gauge theory in $n$-dimensional space. When $\mathcal{R}=n \mathcal{R}$ ep $G, \mathcal{R} \otimes \mathcal{R}^{\text {rev }}$ is an $n \mathrm{~d} G$-gauge theory. When $\mathcal{R}$ describes a higher symmetry, $\mathcal{R} \underset{\text { bulk }(\mathcal{R})}{\otimes} \mathcal{R}^{\text {rev }}$ is a higher gauge theory, but when $\mathcal{R}$ describes an algebraic higher symmetry, $\mathcal{R} \underset{\text { bulk }(\mathcal{R})}{\otimes} \mathcal{R}^{\text {rev }}$ is something new, which is called a gauge theory from "gauging" the algebraic higher symmetry $\mathcal{R}$ in a product state.

When $\mathcal{C}=\mathcal{R}$ and $\hat{\gamma}=\hat{\alpha} \neq$ trivial, the resulting state $\mathcal{R} \underset{\text { bulk }(\mathcal{R})}{\otimes} \hat{\gamma} \underset{\text { bulk }(\mathcal{R})}{\otimes} \mathcal{R}^{\text {rev }}$ in Fig. 30(b) is a twisted $\mathcal{R}$-gauge theory, obtained from "gauging" the algebraic higher symmetry $\mathcal{R}$ in a SPT state stacked with trivial or nontrivial invertible topological order, so gauged SPT states stacked with trivial or nontrivial invertible topological order are classified by $(\mathcal{R}, \hat{\alpha})$. If we just consider gauged SPT states, by ignoring the stacked with trivial or nontrivial invertible topological order, we can replace the invertible domain wall $\hat{\alpha}$ by its induced 


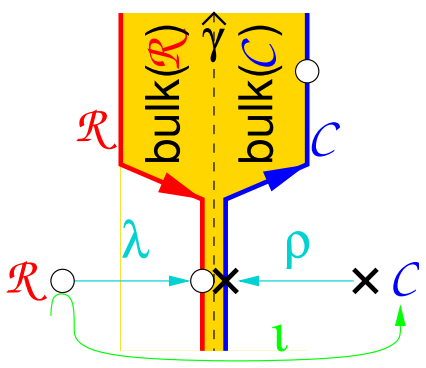

FIG. 31. The data $\mathcal{R} \stackrel{\beta}{\rightarrow} \mathcal{V}, \mathcal{R} \stackrel{\iota}{\hookrightarrow} \mathcal{C}$, and $\operatorname{bulk}(\mathcal{R}) \stackrel{\hat{\mathcal{\nu}}}{\simeq} \operatorname{bulk}(\mathcal{C})$ classify gapped liquid phases (SET or SPT phases) with generalized algebraic higher symmetry $\mathcal{R}$, provided that invertible domain wall $\hat{\gamma}$ is chosen (see Proposition 43) to make the stacking $\mathcal{R} \underset{\text { bulk }(\mathcal{R})}{\otimes}$ $\hat{\gamma} \underset{\text { bulk }(\mathcal{C})}{\otimes} \mathcal{C}^{\text {rev }}$ to describe the gauging of the $\mathcal{R}$-symmetry. The con-

densations of the excitations in $\operatorname{bulk}(\mathcal{R})$ and $\operatorname{bulk}(\mathcal{C})$ form the $\mathcal{R}$ and $\mathcal{C}$ boundaries, which leave parts of the two categorical symmetries, $\operatorname{bulk}(\mathcal{R})$ and bulk $(\mathcal{C})$, unbroken. $\hat{\gamma}$ is chosen so that the two unbroken parts of the two categorical symmetries match with the same $\mathcal{R}$, giving rise to an unbroken $\mathcal{R}$ symmetry.

automorphism $\alpha$ of $Z_{1}(\mathcal{R})$. We see that gauged SPT states are described by $\left(\mathcal{R}, \alpha: Z_{1}(\mathcal{R}) \simeq Z_{1}(\mathcal{R})\right.$ ) (see Fig. 7, where $\mathcal{R}$ is replaced by $\Sigma \mathcal{R})$.

When $\mathcal{C} \neq \mathcal{R}$, the resulting state $\mathcal{R} \underset{\operatorname{bulk}(\mathcal{R})}{\otimes} \hat{\gamma} \underset{\operatorname{bulk}(\mathcal{C})}{\otimes} \mathcal{C}^{\text {rev }}$ in Fig. 30(a) is a topological order obtained from "gauging" the algebraic higher symmetry $\mathcal{R}$ in the gapped liquid state (SET or SPT state) characterized by data $(\mathcal{C}, \iota: \mathcal{R} \hookrightarrow \mathcal{C}, \hat{\gamma}$ : $\operatorname{bulk}(\mathcal{R}) \simeq \operatorname{bulk}(\mathcal{C}))$.

Remark 10. We note that the algebra isomorphism $\mu$ : $\gamma\left(A_{\widetilde{\mathcal{R}}}\right) \simeq A_{\widetilde{\mathcal{R}}_{\mathcal{C}}}$ and the equivalence functor $\widetilde{\gamma}: \widetilde{\mathcal{R}} \simeq \widetilde{\mathcal{R}}_{\mathcal{C}}$ are similar data that are additional to $\gamma: Z_{1}(\mathcal{R}) \simeq Z_{1}(\mathcal{C})$. The fact that these additional data are not manifestly visible in the gauged theory may suggest that they are fixed by $\gamma$ up to certain natural higher structures (such as lower dimensional SPT or invertible phases). As an analogy, $\mu$ or $\tilde{\gamma}$ are similar to the $n$-coboundaries generated by $(n-1)$-cochains that should be mod out when considering the $n$th cohomology., but the exact physical meaning of $\mu$ and $\tilde{\gamma}$ is unclear to us for now. Moreover, $\mu$ and $\tilde{\gamma}$ may need to satisfy some additional conditions that involve even higher structures, and so forth until the top morphisms. The study of these higher structures is beyond our current scope and will be left for future work.

\section{Third version of classification based on gauging the $\mathcal{R}$-symmetry}

We can also use the gauging of the $\mathcal{R}$-symmetry, and the resulting topological order $\mathcal{R} \underset{\text { bulk }(\mathcal{R})}{\otimes} \hat{\gamma} \underset{\text { bulk }(\mathcal{C})}{\otimes} \mathcal{C}^{\text {rev }}$ in Fig. 31 to obtain $\hat{\gamma}$ that keeps the $\mathcal{R}$ part in $\operatorname{bulk}(\mathcal{R})$ unchanged, which leads to another version of classification. We note that the excitations in the topological order $\mathcal{R} \underset{\operatorname{bulk}(\mathcal{R})}{\otimes} \hat{\gamma} \underset{\operatorname{bulk}(\mathcal{C})}{\otimes} \mathcal{C}^{\text {rev }}$ is described a fusion $n$-category $\mathcal{R} \underset{Z_{1}(\mathcal{R})}{\otimes} \gamma \underset{Z_{1}(\mathcal{C})}{\otimes} \mathcal{C}^{\text {rev }}$, where $\gamma$ is a braided equivalence $Z_{1}(\mathcal{R}) \stackrel{\nu}{\simeq} Z_{1}(\mathcal{C})$ induced by the invertible domain wall $\hat{\gamma}$. To make sense of the above statement, let us consider the natural functors from $\mathcal{R}$ to $\mathcal{R} \underset{Z_{1}(\mathcal{R})}{\otimes} \hat{\gamma} \underset{Z_{1}(\mathcal{C})}{\otimes} \mathcal{C}^{\text {rev }}$,

$$
\begin{aligned}
\lambda: \mathcal{R} & \rightarrow \mathcal{R} \underset{Z_{1}(\mathcal{R})}{\otimes} \hat{\gamma} \underset{Z_{1}(\mathcal{C})}{\otimes} \mathcal{C}^{\mathrm{rev}}, \\
x & \mapsto x \underset{Z_{1}(\mathcal{R})}{\otimes} \mathbf{1}_{\hat{\gamma}} \underset{Z_{1}(\mathcal{C})}{\otimes} \mathbf{1}_{\mathcal{C}},
\end{aligned}
$$

and from $\mathcal{C}$ to $\mathcal{R} \underset{Z_{1}(\mathcal{R})}{\otimes} \hat{\gamma} \underset{Z_{1}(\mathcal{C})}{\otimes} \mathcal{C}^{\text {rev }}$

$$
\begin{aligned}
\rho: \mathcal{C} & \rightarrow \mathcal{R} \underset{Z_{1}(\mathcal{R})}{\otimes} \hat{\gamma} \underset{Z_{1}(\mathcal{C})}{\otimes} \mathcal{C}^{\mathrm{rev}}, \\
x & \mapsto \mathbf{1}_{\mathcal{R}} \otimes_{Z_{1}(\mathcal{R})}^{\otimes} \mathbf{1}_{\hat{\gamma}} \otimes_{Z_{1}(\mathcal{C})} x .
\end{aligned}
$$

Here $\lambda$ means mapping from the "left" boundary and $\rho$ means mapping from the "right" boundary ( $\rho$ may not be monoidal here, but it is monoidal when restricted to a subcategory, as we will show later). The above gives two ways to map $\mathcal{R}$ into $\mathcal{R} \underset{Z_{1}(\mathcal{R})}{\otimes} \hat{\gamma} \otimes_{Z_{1}(\mathcal{C})} \mathcal{C}^{\text {rev }}$, namely $\lambda$ and $\rho \circ \iota$. They correspond to observing the $\mathcal{R}$ symmetry from the left $\mathcal{R}$ boundary and from the right $\mathcal{C}$ boundary as in Fig. 31. Thus, we expect that $\lambda$ and $\rho \circ \iota$ coincide. However, recall that in (155) $\hat{\gamma}$ is only required to preserve the "breakable" symmetry with respect to $\beta: \mathcal{R} \rightarrow \mathcal{V}$. Similarly, we only require the breakable symmetry to agree on left and right boundaries of the gauged theory. Let $\operatorname{ker} \beta$ be the preimage of the trivial excitation in $\mathcal{V}$. More precisely, if condensing $A_{\beta}$ gives $\beta: \mathcal{R} \rightarrow \mathcal{V}\left(A_{\beta}\right.$ consists of all the excitations that becomes trivial in $\mathcal{V}), \operatorname{ker} \beta$ is the smallest fusion subcategory of $\mathcal{R}$ containing $A_{\beta}$. $\operatorname{ker} \beta$ is then the breakable symmetry. The restriction $\left.\lambda\right|_{\operatorname{ker} \beta}$ and $\left.(\rho \circ \iota)\right|_{\operatorname{ker} \beta}$ should agree.

Besides, there is a natural half-braiding between the excitations from the left boundary and those from the right boundary. After mapping into the gauged theory,

$$
\lambda(x) \otimes \rho(y) \simeq \rho(y) \otimes \lambda(x) .
$$

On the image $\lambda(\operatorname{ker} \beta)=\rho \circ \iota(\operatorname{ker} \beta)$, the above further defines a braiding:

$$
\begin{aligned}
& \lambda(x) \otimes \lambda(y)=\lambda(x) \otimes \rho(\iota(y)) \\
& \simeq \rho(\iota(y)) \otimes \lambda(x)=\lambda(y) \otimes \lambda(x) .
\end{aligned}
$$

Such braiding makes $\rho$ a monoidal functor when restricted to $\iota(\operatorname{ker} \beta)$. We require the above braiding to be trivial, in the sense that there exists a braided monoidal functor $\lambda(\operatorname{ker} \beta) \rightarrow$ $n$ Vec.

These considerations lead to another version of classification (see Fig. 31):

Proposition 43. Let $\mathcal{R}$ with $\beta: \mathcal{R} \rightarrow \mathcal{V}$ be a $\mathcal{V}$-local fusion $n$-category. Anomaly-free gapped liquid phases in $n$ dimensional space with an anomaly-free algebraic higher symmetry $\mathcal{R}$ are classified by data $(\mathcal{R} \stackrel{\iota}{\hookrightarrow} \mathcal{C}, \hat{\gamma})$, where $\mathcal{C}$ is a fusion $n$-category that includes $\mathcal{R}$ (i.e., $\iota: \mathcal{R} \stackrel{\iota}{\hookrightarrow} \mathcal{C}$ is a top-fully faithful functor), and $\hat{\gamma}: \operatorname{bulk}(\mathcal{R}) \simeq \operatorname{bulk}(\mathcal{C})$ is an invertible domain wall between $\operatorname{bulk}(\mathcal{R})$ and $\operatorname{bulk}(\mathcal{C})$. $\hat{\gamma}$ induces a braided equivalence $\gamma: Z_{1}(\mathcal{R}) \stackrel{\gamma}{\simeq} Z_{1}(\mathcal{C})$ such that the following diagram is commutative (up to a natural 
isomorphism),

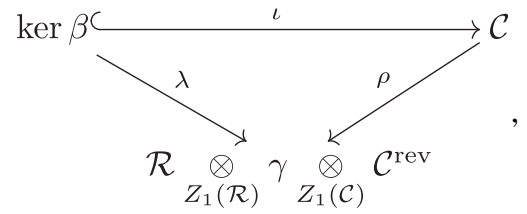

and the braiding in the image $\lambda(\operatorname{ker} \beta)$ defined above is trivial.

When $\mathcal{C}=\mathcal{R}$, the above gives a classification of SPT phases with symmetry $\mathcal{R}$.

\section{A simple example for $\mathbb{Z}_{2} \times \mathbb{Z}_{2}$ symmetry in 1-dimensional space}

We would like to apply the above results to compute the $\mathbb{Z}_{2} \times \mathbb{Z}_{2}$ SPT phases in 1-dimensional space. This leads to deeper understanding of SPT order.

Let $\mathcal{R}=\mathcal{R} \operatorname{ep}\left(\mathbb{Z}_{2} \times \mathbb{Z}_{2}\right), \beta: \mathcal{R} \rightarrow \mathcal{V e c}$ be the forgetful functor, $\widetilde{\mathcal{R}}=\mathcal{V ~ e c}_{\mathbb{Z}_{2} \times \mathbb{Z}_{2}}$, and $\mathcal{M}=Z_{1}(\mathcal{R})$. We would like to compute the automorphisms of $\mathcal{M}$ that preserves the embedding $\iota_{\mathcal{R}}: \mathcal{R} \hookrightarrow \mathcal{M}$ or the bulk-to-boundary functor $F_{\tilde{\mathcal{R}}}$ : $\mathcal{M} \rightarrow \widetilde{\mathcal{R}}$.

$\mathcal{M}$ is pointed. It is most efficiently represented by a metric group $\left(\mathbb{Z}_{2}^{4}, \theta\right)$, where $\theta$ is a nondegenerate quadratic form which is physically the topological spin. We denote elements in $\mathbb{Z}_{2}^{4}$ by four-component mod 2 integer vectors $(a, b, c, d)$. We pick $\theta$ to be

$$
\theta(a, b, c, d)=(-1)^{a c+b d} .
$$

In other words, $(1,0,1,0)$ and $(0,1,0,1)$ are fermions. If one views $\mathcal{M}$ as a double-layer toric code, the generators are identified as the following:

$$
\begin{aligned}
(1,0,0,0) \sim e \mathbf{1}, & (0,1,0,0) \sim \mathbf{1} e, \\
(0,0,1,0) \sim m \mathbf{1}, & (0,0,0,1) \sim \mathbf{1} m .
\end{aligned}
$$

$\mathcal{R}=\mathcal{R e p}\left(\mathbb{Z}_{2} \times \mathbb{Z}_{2}\right)$ is generated by four simple objects $\mathbf{1 1}, e \mathbf{1}, \mathbf{1} e, e e$. Thus, the embedding is

$$
\iota_{\mathcal{R}}(a, b)=(a, b, 0,0) .
$$

$\widetilde{\mathcal{R}}=\mathcal{V e c}_{\mathbb{Z}_{2} \times \mathbb{Z}_{2}}$ is generated by four simple objects 11, $m \mathbf{1}, \mathbf{1} \mathrm{m}, \mathrm{mm}$. Thus, the bulk-to-boundary functor is

$$
F_{\widetilde{\mathcal{R}}}(a, b, c, d)=(c, d) .
$$

An automorphism of $\mathcal{M}$ is the same as a group automorphism $\alpha$ of $\mathbb{Z}_{2}^{4}$ that preserves $\theta$, i.e.,

$$
\theta(\alpha(a, b, c, d))=(-1)^{a c+b d} .
$$

Case 1. $\alpha$ preserves embedding as in (150):

We require that

$$
\alpha(a, b, 0,0)=(a, b, 0,0) .
$$

Thus,

$$
\alpha(a, b, c, d)=(a, b, 0,0)+\alpha(0,0, c, 0)+\alpha(0,0,0, d) .
$$

Let $\quad \alpha(0,0, c, 0)=c\left(x_{1}, x_{2}, x_{3}, x_{4}\right) \quad$ and $\quad \alpha(0,0,0, d)=$ $d\left(y_{1}, y_{2}, y_{3}, y_{4}\right)$. Since $\alpha$ should preserve spin (174), we have

$$
\begin{aligned}
a c+b d= & \left(a+c x_{1}+d y_{1}\right)\left(c x_{3}+d y_{3}\right) \\
& +\left(b+c x_{2}+d y_{2}\right)\left(c x_{4}+d y_{4}\right) \bmod 2 .
\end{aligned}
$$

Rearrange the terms to obtain

$$
\begin{aligned}
& a c\left(1+x_{3}\right)+a d y_{3}+b d\left(1+y_{4}\right)+b c x_{4} \\
& +c^{2}\left(x_{1} x_{3}+x_{2} x_{4}\right)+d^{2}\left(y_{1} y_{3}+y_{2} y_{4}\right) \\
& +c d\left(x_{1} y_{3}+x_{3} y_{1}+x_{2} y_{4}+x_{4} y_{2}\right)=0 \bmod 2 .
\end{aligned}
$$

One must have $x_{3}=y_{4}=1, y_{3}=x_{4}=0$. Then

$$
c^{2} x_{1}+d^{2} y_{2}+c d\left(y_{1}+x_{2}\right)=0 \bmod 2 .
$$

Thus, $x_{1}=y_{2}=0, y_{1}=x_{2}$. We got two solutions:

$$
\begin{gathered}
\alpha_{0}(a, b, c, d)=(a, b, c, d), \\
\alpha_{1}(a, b, c, d)=(a+d, b+c, c, d) .
\end{gathered}
$$

Case 2. $\alpha$ preserves bulk-to-boundary functor as in (155): Now we require that

$$
F_{\widetilde{\mathcal{R}}} \alpha(a, b, c, d)=(c, d) .
$$

In other words,

$$
\alpha(a, b, c, d)=(*, *, c, d) .
$$

Let $\alpha(a, 0,0,0)=a\left(p_{1}, p_{2}, 0,0\right), \alpha(0, b, 0,0)=b\left(q_{1}, q_{2}, 0,0\right)$, $\alpha(0,0, c, 0)=c\left(r_{1}, r_{2}, 1,0\right), \quad \alpha(0,0,0, d)=d\left(s_{1}, s_{2}, 0,1\right)$. $\alpha$ preserves spin (174) and gives

$$
\begin{aligned}
a c+b d= & \left(a p_{1}+b q_{1}+c r_{1}+d s_{1}\right) c \\
& +\left(a p_{2}+b q_{2}+c r_{2}+d s_{2}\right) d \bmod 2 .
\end{aligned}
$$

One must have $p_{2}=q_{1}=r_{1}=s_{2}=0, p_{1}=q_{2}=1$, and $s_{1}=r_{2}$. We also have two solutions:

$$
\begin{gathered}
\alpha_{0}(a, b, c, d)=(a, b, c, d), \\
\alpha_{1}(a, b, c, d)=(a+d, b+c, c, d) .
\end{gathered}
$$

We see that the two approaches indeed give rise to the same solutions.

Although for pointed modular tensor categories (metric groups), the automorphism is fully determined by the map on objects, it is not the case for the automorphisms on fusion categories. Below, we briefly explain the nontrivial structures of $\widetilde{\alpha}: \widetilde{\mathcal{R}} \rightarrow \widetilde{\mathcal{R}}$. By Lemma 2.1.5 in Ref. [92], we know that $F_{\widetilde{\mathcal{R}}}$ and $F_{\widetilde{\mathcal{R}}} \circ \alpha_{1}$ differ by a nontrivial automorphism $\tilde{\alpha}$ of $\mathcal{R}=\mathcal{V e c}_{\mathbb{Z}_{2} \times \mathbb{Z}_{2}}=\{\mathbf{1 1}, m \mathbf{1}, \mathbf{1} m, m m\}$, corresponding to the nontrivial cohomology class in $H^{2}\left(\mathbb{Z}_{2} \times \mathbb{Z}_{2}, U(1)\right)=$ $\mathbb{Z}_{2}$. Such automorphism is identity on objects $\widetilde{\alpha}(g)=g$ but has nontrivial tensor structures, namely $\widetilde{\alpha}(g) \otimes \widetilde{\alpha}(h) \stackrel{\omega(g, h)}{\longrightarrow}$ $\widetilde{\alpha}(g h)$, where $\omega(g, h) \in H^{2}\left(\mathbb{Z}_{2} \times \mathbb{Z}_{2}, U(1)\right)$ is nontrivial.

The nontrivial cohomology class in $H^{2}\left(\mathbb{Z}_{2} \times \mathbb{Z}_{2}, U(1)\right)$ can be represented by $\omega\left(\left(c_{1}, d_{1}\right),\left(c_{2}, d_{2}\right)\right)=(-1)^{c_{1} d_{2}}$. We can also see the nontrivial tensor structure of $\alpha_{1}$. Denote the tensor structure of $\alpha_{1}$ by $u(x, y): \alpha_{1}(x) \otimes \alpha_{1}(y) \rightarrow \alpha_{1}(x \otimes y)$. It needs to preserve braiding, namely,

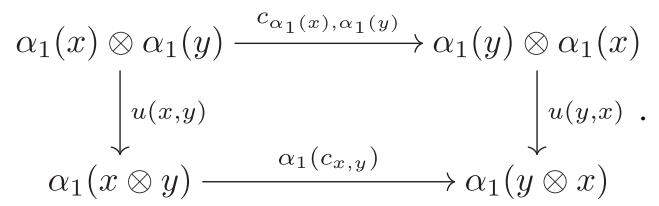


Let $x=(0,0,1,0) \sim m \mathbf{1}$ and $y=(0,0,0,1) \sim \mathbf{1} m . c_{x, y}=1$ since it braids $m$ in different layers. $\alpha_{1}(x)=(0,1,1,0) \sim m e$ and $\alpha_{1}(y)=(1,0,0,1) \sim e m$. Therefore, $c_{\alpha_{1}(x), \alpha_{1}(y)}=-1$ since it means braiding $m$ with $e$ in the first layer and braiding $e$ with $m$ in the second layer, and thus in total a full braiding between $e$ and $m$. Clearly the values of these two special braidings are independent of gauge. We conclude that, independent of gauge, $u((0,0,1,0),(0,0,0,1))=$ $-u((0,0,0,1),(0,0,1,0))$, which means $u$ cannot be cohomologically trivial. It is not hard to check that $u((0,0,1,0),(0,0,0,1))=-u((0,0,0,1),(0,0,1,0))$ agrees with $\omega\left(\left(c_{1}, d_{1}\right),\left(c_{2}, d_{2}\right)\right)=(-1)^{c_{1} d_{2}}$. This way, we show that

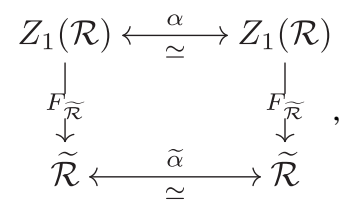

which is an example of Proposition 36.

Next, we examine the condensable algebras $A_{\mathcal{R}}$ and $A_{\widetilde{\mathcal{R}}}$. By definition, $A_{\widetilde{\mathcal{R}}}$ is the direct sum of anyons that maps to trivial under $F_{\widetilde{\mathcal{R}}}$. It is easy to see that

$$
\begin{array}{r}
A_{\widetilde{\mathcal{R}}}=\mathbf{1 1} \oplus e \mathbf{1} \oplus \mathbf{1} e \oplus e e=\oplus_{a b}(a, b, 0,0), \\
A_{\mathcal{R}}=\mathbf{1 1} \oplus m \mathbf{1} \oplus \mathbf{1} m \oplus m m=\oplus_{c d}(0,0, c, d) .
\end{array}
$$

One can check that the overlap of $A_{\widetilde{\mathcal{R}}}$ and $A_{\mathcal{R}}$ is $(0,0,0,0)$, which implies that $\mathcal{R} \otimes \widetilde{\mathcal{R}}^{\text {rev }}=\mathcal{V}$ ec. This result can be verified explicitly using the techniques developed in Refs. [93,94]. Also, $A_{\widetilde{\mathcal{R}}} \otimes A_{\mathcal{R}}=\oplus_{a b c d}(a, b, c, d)$.

It is obvious that an automorphism preserving $A_{\tilde{\mathcal{R}}}$ is the same as preserving the embedding $\mathcal{R} \stackrel{\iota_{\mathcal{R}}}{\longrightarrow} \mathcal{M}$, and also the same as preserving the bulk-to-boundary functor $F_{\widetilde{\mathcal{R}}}: \mathcal{M} \rightarrow \widetilde{\mathcal{R}}$.

\section{EMERGENT LOW-ENERGY EFFECTIVE ALGEBRAIC HIGHER SYMMETRY AND CATEGORICAL SYMMETRY}

\section{A. Emergent of categorical symmetry from energy scale separation}

In real $n$ d condensed matter systems, we usually have 0 -symmetry described by a group $G$ and the associated categorical symmetry $\mathrm{M}=\operatorname{bulk}(n \mathcal{R} \operatorname{ep} G)$ (which is also denoted as $G \vee \widetilde{G}^{(n-1)}$ ), but it is hard to have higher symmetry and algebraic higher symmetries, unless we fine-tune the lattice model (if we do not include dynamical electromagnetic field [16]). However, emergent algebraic higher symmetries and associated categorical symmetries can appear at low energies, if our models have an energy scale separation [16]. This is a practical way to realize algebraic higher symmetries and associated categorical symmetries, which makes the results of this paper useful.

In this subsection, we will discuss how to compute the emergent algebraic higher symmetries and the categorical symmetries. It turns out we just need to compute the emergence of categorical symmetries $\mathrm{M}$. The emergent algebraic higher symmetries $\mathcal{R}$ can be determined from the emergent categorical symmetries directly, by solving two equations $\operatorname{bulk}(\mathcal{R}) \simeq \mathrm{M}$ and $\mathcal{R} \stackrel{\beta}{\rightarrow} n \mathcal{V}$ ec. The solutions are usually not unique, but the different solutions are holo-equivalent.

Let us consider a gapped liquid state in $n$-dimensional lattice. We assume the excitations in the gapped state has a large separation of energy scale. The low-energy excitations (point-like, string-like, etc.) are closed under fusion and form a fusion $n$-category $\mathcal{C}^{\text {low }}$. All other topological excitations have very high energies, which are assumed to be infinite. Now we add interactions among those low-energy excitations to drive phase transitions by condensing the low-energy excitations to form gapless states, etc. We assume that, in such a process, the high-energy excitations still have high energies (i.e., infinite energy). What are the possible phases and gapless states?

Some constraints to the low-energy physics come from the underlying symmetry, while other constraints come from the fusion and statistics of those low-energy topological excitations. It looks hard to understand the effects of all those different constraints, but it turns out that the holographic point of view and the associated categorical symmetry can help us to solve this problem.

We know that some excitations in $\mathcal{C}^{\text {low }}$ are topological excitations, while others are charge objects of the underlying symmetry. To use the holographic point of view and to use categorical symmetry, we restrict discussion to the symmetric sub-Hilbert space of the underlying symmetry. In this case, every excitations in $\mathcal{C}^{\text {low }}$ can be viewed as topological excitations in a hypothetical system without symmetry. However, the fusion $n$-category $\mathcal{C}^{\text {low }}$ that describes those excitations is in general anomalous; i.e., it cannot be realized by a lattice system in the same dimension without symmetry, but it can be realized as a boundary of a topological order $\mathrm{M}^{\text {low }}=$ $\operatorname{bulk}\left(\mathcal{C}^{\text {low }}\right)$ in one higher dimension [see (43)]. In fact, $\mathrm{M}^{\text {low }}$ is nothing but the emergent categorical symmetry, which provides all the constraints to the low energy physics and solves our problem.

We see that the only input is the low-energy excitations $\mathcal{C}^{\text {low }}$, so we do not need to have a lattice model. The above discussion remains valid for field theories without a given or known lattice regularization. (In this paper, we use the term field theory to mean theory without a given or known lattice regularization.)

Proposition 44. For a lattice system or a field theory with low-energy excitations $\mathcal{C}^{\text {low }}$, the system has a low-energy effective (i.e., emergent) categorical symmetry given by $\mathrm{M}^{\text {low }}=$ $\operatorname{bulk}\left(\mathcal{C}^{\text {low }}\right)$ that provides all the constraints to the low-energy physics.

Such a low-energy effective categorical symmetry $M^{\text {low }}$ is present even when low-energy excitations condense, undergo phase transitions, etc., as long as all other higher energy excitations have very high energies. The emergent categorical symmetry controls all the low-energy behaviors of the system, including allowed phases, allowed phase transitions, allowed critical points, etc. This is because the allowed phases, allowed phase transitions, allowed critical points, etc. one-to-one correspond to different boundaries of $\mathrm{M}^{\text {low }}$, the categorical symmetry. In some sense, $M^{\text {low }}$ is a "topological invariant" of low-energy physics and, we believe, is a 
complete topological invariant. All other low-energy topological invariants can be obtained from $\mathrm{M}^{\text {low }}$.

Such an emergent categorical symmetry is the most practical and useful application of the notion of categorical symmetry and the holographic point of view. For example, consider Proposition 45.

Proposition 45. Consider a gapped liquid state in $n$ dimensional space whose low-energy energy excitations are described by a fusion $n$-category $\mathcal{C}^{\text {low }}$. When all other excitations have higher energies, the gapped liquid phases formed by low-energy excitations in $\mathcal{C}^{\text {low }}$ must have excitations described by a fusion $n$-category $\mathcal{C}$ that satisfies $\operatorname{bulk}(\mathcal{C}) \simeq$ $\operatorname{bulk}\left(\mathcal{C}^{\text {low }}\right)$.

In fact, $\operatorname{bulk}(\mathcal{C}) \simeq \operatorname{bulk}\left(\mathcal{C}^{\text {low }}\right)$ is nothing but the anomaly matching condition, since the categorical symmetries bulk $(\mathcal{C})$ and bulk $\left(\mathcal{C}^{\text {low }}\right)$, as topological orders in one higher dimension, are the effective noninvertible gravitational anomalies [3,25], after we view the charge objects of the symmetry as topological excitations.

Remark 11. We like to point out that the effective gravitational anomaly here is more general then the usual gravitational anomaly from the noninvariance of the path integral. The usual gravitational anomaly is invertible, while our effective gravitational anomaly, as topological order in one higher dimension, is in general noninvertible [24-26]. Since the usual gravitational anomaly is invertible, it corresponds to invertible topological order in one higher dimension, which contains no nontrivial topological excitations. Thus, the usual gravitational anomaly does not encode any conservation law, since the conservation law must come from the fusion rule of excitations for the topological order in one higher dimension. In contrast, a noninvertible gravitational anomaly does encode a conservation law, since its corresponding topological order in one higher dimension has nontrivial excitations and nontrivial fusion rule. Therefore, a noninvertible gravitational anomaly can be viewed as a symmetry. This is why we also refer to a noninvertible gravitational anomaly as categorical symmetry, to stress its connection to symmetry.

\section{B. States with the full categorical symmetry}

Since all the gapped liquid states in systems with an (emergent) categorical symmetry must spontaneously break part of the categorical symmetry, the states with the full unbroken categorical symmetry must be gapless. A system with a categorical symmetry $\mathrm{M}$ may have many different symmetric gapless states. Those gapless states may have additional emergent categorical symmetry. So what is the minimal gapless state with the categorical symmetry $M$ ? To define the notion of "minimal gapless state" in $n$-dimensional space, we assume that the gapless excitations all have the same linear dispersion $\omega=v k$. The low-temperature specific heat of the gapless state has a form

$$
c_{V}=c \gamma_{n} T^{n}
$$

where

$$
\gamma_{n}=(n+1) k_{B}\left(\frac{k_{B}}{v}\right)^{n} \int \frac{d^{n} \boldsymbol{k}}{(2 \pi)^{n}} \frac{|\boldsymbol{k}|}{e^{|\boldsymbol{k}|}-1} .
$$

For a system described by a single gapless real scalar field, we find that $c=1$. The minimal gapless state has minimal $c$.
From the above discussions, we see that minimal gapless states with the categorical symmetry $M$ are actually minimal gapless boundary of topological order with excitations described by $\mathrm{M}$ in one higher dimension. References [24,61,62] discussed how to obtain gapless boundaries for $2 \mathrm{~d}$ topological orders, using modular covariant partition functions or topological Wick rotation. Those gapless boundaries do not break the categorical symmetry $\mathrm{M}$. Those approaches also allow us to obtain the minimal gapless boundaries with minimal central charge. However, for a given categorical symmetry, it is not clear whether its minimal gapless state is unique or not [19].

\section{EXAMPLES}

In the section, we discuss some gapped liquid phases. In particular, we identify their algebraic higher symmetry and categorical symmetry. We also discuss low-energy effective (i.e., emergent) categorical symmetry when some topological excitations have low energies.

\section{A. The category of $0 \mathrm{~d}$ topological orders}

The category of $0 \mathrm{~d}$ topological orders $\mathrm{TO}^{1}$ is the category of Od gapped phases with no symmetry. In 0d, a stable gapped phase has nondegenerate ground state, which corresponds to a simple object in the category of 0d gapped phases, denoted as $\mathrm{TO}^{1}$. This is the only simple object in $\mathrm{TO}^{1}$ and is the unit object of stacking operation $\otimes$, which is the tensor product of vector spaces. We denote this unit object as $\mathbf{1}$. There are accidental degenerate ground states, which corresponds to a composite object $\underbrace{\mathbf{1} \oplus \mathbf{1} \oplus \cdots \oplus \mathbf{1}}_{m \text { copies }}=m \mathbf{1}$. In $\mathrm{TO}^{1}$, a 1 morphism from $m \mathbf{1}$ to $n \mathbf{1}$ is an $n \times m$ complex matrix $M$ : $m \mathbf{1} \stackrel{M}{\rightarrow} n \mathbf{1}$. Such a fusion 1 -category happen to be $1 \mathcal{V}$ ec. We see that $\mathrm{TO}^{1}=1 \mathcal{V}$ ec $\equiv \mathcal{V}$ ec.

\section{B. The $2 d$ topological order described by $\mathbb{Z}_{2}$ gauge theory}

The $2 \mathrm{~d} \mathbb{Z}_{2}$ topological order described by the $\mathbb{Z}_{2}$ gauge theory is denoted by $\mathrm{GT}_{\mathbb{Z}_{2}}^{3}$. Codimension- 2 excitations are described by the following braided fusion 1-category $\Omega^{2} \mathrm{GT}_{\mathbb{Z}_{2}}^{3}$, which has four simple objects (the point-like excitations): $\mathbf{1}, e, m, f$ with the following $\mathbb{Z}_{2}$ fusion rule:

$$
e \otimes e=m \otimes m=f \otimes f=\mathbf{1},
$$

where $\mathbf{1}$ is the trivial excitation. $e, m, f$ are topological excitations which have mutual $\pi$-statistics between them. $e, m$ are bosons, and $f$ is a fermion. Such a topological order $\mathrm{GT}_{\mathbb{Z}_{2}}^{3}$ can be realized by lattice models in the same dimension (see Refs. [5,81,82]). Therefore, the bulk of $\mathrm{GT}_{\mathbb{Z}_{2}}^{3}$ is a $3 \mathrm{~d}$ product state, i.e., Bulk $\left(G_{\mathbb{Z}_{2}}^{3}\right)=I^{4}$ [see (41)]. The $2 d$ topological order $\mathrm{GT}_{\mathbb{Z}_{2}}^{3}$ has no categorical symmetry since $\operatorname{Bulk}\left(\mathrm{GT}_{\mathbb{Z}_{2}}^{3}\right)=$ $\mathrm{I}^{4}$ or $\operatorname{bulk}\left(\Omega G \mathrm{~T}_{\mathbb{Z}_{2}}^{3}\right)=\mathrm{I}^{4}$.

Next, we consider the situation when $e$ particles have low energies, and $m, f$ particles have very high energies. The lowenergy excitations form a fusion 2-category $2 \mathcal{R} \operatorname{ep} \mathbb{Z}_{2}$ (after condensation completion), which simply describes $2 \mathrm{~d}$ bosons with mod-2 conservation. In this limit, we have a low-energy effective categorical symmetry characterized by the $3 \mathrm{~d} \mathbb{Z}_{2}$ gauge theory $\mathrm{GT}_{\mathbb{Z}_{2}}^{4}=\operatorname{bulk}\left(2 \mathcal{R} \operatorname{ep} \mathbb{Z}_{2}\right)$. 
$2 \mathcal{R}$ ep $\mathbb{Z}_{2}$ describes the excitations in a system with $\mathbb{Z}_{2}$ symmetry in the $\mathbb{Z}_{2}$ symmetric phase (within the symmetric sub-Hilbert space). $2 \mathcal{R}$ ep $\mathbb{Z}_{2}$ also describes the excitations in one of the gapped boundaries of $3 \mathrm{~d} \mathbb{Z}_{2}$ gauge theory $\mathrm{GT}_{\mathbb{Z}_{2}}^{4}$, obtained by condensing the $\mathbb{Z}_{2}$-flux lines in $\mathrm{GT}_{\mathbb{Z}_{2}}^{4}$ at the boundary. The $3 \mathrm{~d} \mathbb{Z}_{2}$ gauge theory $\mathrm{GT}_{\mathbb{Z}_{2}}^{4}$ has another gapped boundary whose excitations are described by the fusion 2-category $2 \mathcal{V} \mathbb{C}_{\mathbb{Z}_{2}}$ (a $\mathbb{Z}_{2}$ symmetry-breaking phase with $e$ boson condensation), obtained from condensing the $\mathbb{Z}_{2}$ charges in $\mathrm{GT}_{\mathbb{Z}_{2}}^{4}$ at the boundary. The second boundary corresponds to another gapped phase of the system with the $\mathbb{Z}_{2}$ symmetry - the spontaneous symmetry-breaking phase. The continuous phase transition between the two gapped phases is described by a critical point which has the full categorical symmetry characterized by $\mathrm{GT}_{\mathbb{Z}_{2}}^{4}$ (the $3 \mathrm{~d} \mathbb{Z}_{2}$ gauge theory). This critical point is the same as the critical point of $2 \mathrm{~d}$ quantum Ising model (or 3d statistical Ising model), which has the same categorical symmetry $\mathrm{GT}_{\mathbb{Z}_{2}}^{4}$, as discussed in Ref. [19].

When $e$ bosons have low energies, the resulting $\mathbb{Z}_{2}$ symmetric system can have infinity many different symmetric gapped phases, and one of them is the $2 \mathrm{~d} \mathbb{Z}_{2}$-SPT phase. The critical point at the continuous transition from the $\mathbb{Z}_{2}$ SPT phase to the $\mathbb{Z}_{2}$ spontaneous symmetry-breaking phase is described by the same critical point discussed above; this is because the transition is also described by the same $\mathbb{Z}_{2}$ charge condensation.

Last, we consider the situation when $f$ particles have low energies and $e, m$ particles have very high energies. The low-energy excitations form a fusion 2-category $2 \mathrm{~s} \mathcal{V}$ ec, which simply describes $2 \mathrm{~d}$ fermions with mod- 2 conservation. There is a $\mathbb{Z}_{2}^{f}$ symmetry from the mod-2 conservation of the fermions. In this limit, we have a low-energy effective categorical symmetry characterized by $3 \mathrm{~d}$ twisted $\mathbb{Z}_{2}$ gauge theory with fermionic $\mathbb{Z}_{2}$ charge, denoted by $\mathrm{GT}_{\mathbb{Z}_{2}^{f}}^{4}$. (The $3 \mathrm{~d}$ twisted $\mathbb{Z}_{2}$ gauge theory $\mathrm{GT}_{\mathbb{Z}_{2}^{f}}^{4}$ is obtained by gauging $\mathbb{Z}_{2}^{f}$, a $\mathbb{Z}_{2}$ symmetry with fermionic $\mathbb{Z}_{2}$ charge.) The categorical symmetry $\mathrm{GT}_{\mathbb{Z}_{2}^{f}}^{4}$ is different from the categorical symmetry $\mathrm{GT}_{\mathbb{Z}_{2}}^{4}$ discussed above. So when $f$ fermions have low energies, our system has different properties from when $e$ bosons have low energies.

When $f$ fermions have low energies, our system can have 16 gapped phases (up to $E_{8} 2 \mathrm{~d}$ bosonic invertible topological order) labeled by $\alpha \in \mathbb{Z}_{16}$, which correspond to $2 \mathrm{~d}$ fermionic invertible topological orders. The continuous transition between $\alpha$ and $\alpha+1$ phases is described by the following $2 \mathrm{~d}$ noninteracting Majorana fermion theory $[95,96]$ :

$$
\begin{aligned}
& H=\int d^{2} \boldsymbol{x}\left[\lambda^{\top}(\boldsymbol{x}) \gamma^{i} \partial_{i} \lambda(\boldsymbol{x})+m \lambda^{\top}(\boldsymbol{x}) i \sigma^{2} \lambda(\boldsymbol{x})\right], \\
& \lambda=\left(\begin{array}{c}
\lambda_{1} \\
\lambda_{2}
\end{array}\right), \quad \lambda^{*}=\lambda, \quad \gamma^{1}=\sigma^{1}, \quad \gamma^{2}=\sigma^{3} .
\end{aligned}
$$

where $\sigma^{i}$ is the Pauli matrix. The transition happens when $m$ changes sign, which changes the chiral central charge of the edge state by $1 / 2[95,96]$. The gapless state at $m=0$ has the full categorical symmetry $\mathrm{GT}_{\mathbb{Z}_{2}^{f}}^{4}$.

\section{The 3d topological order described by $\mathbb{Z}_{2}$ gauge theory}

The $3 \mathrm{~d} \mathbb{Z}_{2}$ topological order $\mathrm{GT}_{\mathbb{Z}_{2}}^{4}$ (described by the $\mathbb{Z}_{2}$ gauge theory) has codimension- 2 and codimension- 3 excitations described by the braided fusion 2-category $\Omega^{2} \mathrm{GT}_{\mathbb{Z}_{2}}^{4}$ : The simple objects (the string-like excitations) are labeled by $\mathbf{1}_{s}, m_{s}, e_{s}, m_{s} \otimes e_{s}$, with the following symmetric fusion:

$$
\begin{aligned}
\mathbf{1}_{s} \otimes m_{s}=m_{s}, & \mathbf{1}_{s} \otimes e_{s}=e_{s}, \\
m_{s} \otimes m_{s}=\mathbf{1}_{s}, & e_{s} \otimes e_{s}=2 e_{s},
\end{aligned}
$$

where $\mathbf{1}_{s}$ is the trivial string. $m_{s}$ is a bosonic topological stringlike excitation that corresponds to the $\mathbb{Z}_{2}$-flux string.

The simple 1-morphisms (the pointlike excitations), that connect $\mathbf{1}_{s} \rightarrow \mathbf{1}_{s}$, are labeled by $\mathbf{1}_{p}, e_{p}$, with the following $\mathbb{Z}_{2}$ fusion:

$$
e_{p} \otimes e_{p}=\mathbf{1}_{p},
$$

where $\mathbf{1}_{p}$ is the trivial particle. $e_{p}$ is a bosonic topological excitation with trivial mutual statistics. However, $e_{p}$ and $m_{s}$ have a nontrivial mutual $\pi$-statistics between them. We also have simple 1-morphisms that connect $m_{s} \rightarrow m_{s}$, which are labeled by $\mathbf{1}_{m_{s}}, e_{m_{s}}$ with the following $\mathbb{Z}_{2}$ fusion:

$$
e_{m_{s}} \otimes e_{m_{s}}=\mathbf{1}_{m_{s}} .
$$

They correspond to the point-like excitations on the string $m_{s}$.

The $e_{s}$ string mentioned above is a descendent excitation, formed by condensing $e_{p}$ point-like excitations along the string. Since $e_{p}$ has a mod 2 conservation, the $e_{p}$ condensed state is a spontaneously $\mathbb{Z}_{2}$ symmetry-breaking state. This leads to the fusion rule $e_{s} \otimes e_{s}=2 e_{s}$.

Such a $\mathrm{GT}_{\mathbb{Z}_{2}}^{4}$ topological order has a trivial categorical symmetry since $\operatorname{Bulk}\left(G_{\mathbb{Z}_{2}}^{4}\right)=I^{5}$ or $\operatorname{bulk}\left(\Omega G_{\mathbb{Z}_{2}}^{4}\right)=I^{5}$ (where $\Omega \mathrm{GT}_{\mathbb{Z}_{2}}^{4}$ describes the excitations in $\mathrm{GT}_{\mathbb{Z}_{2}}^{4}$ ). However, when some excitations have low energy and other have high energies, the system may have a low-energy effective categorical symmetry.

When $e_{p}$ particles have low energies and $m_{s}$ strings have very high energies, the low-energy excitations are described by a fusion 3 -category $3 \mathcal{R}$ ep $\mathbb{Z}_{2}$ generated by $e_{p}$ particles. In this limit, the low-energy effective categorical symmetry is $\operatorname{bulk}\left(3 \mathcal{R}\right.$ ep $\left.\mathbb{Z}_{2}\right)=\mathrm{GT}_{\mathbb{Z}_{2}}^{5}$, which is nothing but the $4 \mathrm{~d} \mathbb{Z}_{2}$ gauge theory. Such a categorical symmetry has the following two gapped phases (plus many others):

(1) a phase with low-energy excitations $3 \mathcal{R}$ ep $\mathbb{Z}_{2}$ (corresponding to the symmetric phase of $3 \mathrm{~d}$ quantum Ising model);

(2) a phase with low-energy excitations $3 \mathcal{V e c}_{\mathbb{Z}_{2}}$ (corresponding to the spontaneous symmetry-breaking phase of $3 \mathrm{~d}$ quantum Ising model).

The transition between the two gapped phase is Higgs transition of the $3 \mathrm{~d} \mathbb{Z}_{2}$ gauge theory. The critical point has the full categorical symmetry $\mathrm{GT}_{\mathbb{Z}_{2}}^{5}$. Such a critical point is the same as the critical point in $3 \mathrm{~d}$ quantum Ising model or $4 \mathrm{~d}$ statistical Ising model, which is described by noninteracting massless real scaler field

$$
S=\int d t d^{3} \boldsymbol{x}\left[\frac{1}{2}\left(\partial_{t} \phi\right)^{2}+\frac{1}{2} v^{2}\left(\partial_{x} \phi\right)^{2}\right] .
$$

When $m_{s}$ strings have low energies and $e_{p}$ particles have very high energies, the low-energy excitations are described 
by a fusion 3-category generated by $m_{s}$ strings, which are denoted as $3 \mathcal{R} \operatorname{ep} \mathbb{Z}_{2}^{(1)}$. Ignoring the descendant excitations, $3 \mathcal{R}$ ep $\mathbb{Z}_{2}^{(1)}$ has only a single trivial object, two simple 1morphisms (trivial string $\mathbf{1}_{s}$ and $\mathbb{Z}_{2}$ flux string $m_{s}$ ), and a single trivial 2-morphism. In this limit, the low-energy effective categorical symmetry is bulk $\left(3 \mathcal{R}\right.$ ep $\left.\mathbb{Z}_{2}^{(1)}\right)=\mathrm{GT}_{\mathbb{Z}_{2}^{(1)}}^{5}$, where $\mathrm{GT}_{\mathbb{Z}_{2}^{(1)}}^{5}$ is the $4 \mathrm{~d} \mathbb{Z}_{2}$ 2-gauge theory obtained by gauging $\mathbb{Z}_{2}^{(1)}$ 1-symmetry. The $4 \mathrm{~d} \mathbb{Z}_{2}^{(1)}$ 2-gauge theory has a string-like $\mathbb{Z}_{2}$ charge and string-like $\mathbb{Z}_{2}$ flux. The $\mathbb{Z}_{2}$ string-charge and the $\mathbb{Z}_{2}$ string-flux has mutual $\pi$-statistics. Such a categorical symmetry has two gapped phases:

(1) a phase with low energy excitations $3 \mathcal{R}$ ep $\mathbb{Z}_{2}^{(1)}$;

(2) another phase also with low energy excitations $3 \mathcal{R e p} \mathbb{Z}_{2}^{(1)}$.

The transition between the two phases is the confinement transition of the $3 d \mathbb{Z}_{2}$ gauge theory. The critical point of the transition has the full categorical symmetry $\mathrm{GT}_{\mathbb{Z}_{2}^{(1)}}^{5}$. Such a critical point is different from the Higgs transition critical point which has a categorical symmetry $\mathrm{GT}_{\mathbb{Z}_{2}}^{5}$ (for details, see Ref. [19]).

\section{The 3d topological order described by twisted $\mathbb{Z}_{2}$ gauge theory}

The $3 \mathrm{~d}$ topological order described by the twisted $\mathbb{Z}_{2}$ gauge theory (i.e., $3 \mathrm{~d} \mathbb{Z}_{2}$ gauge theory with fermionic point-like $\mathbb{Z}_{2}$ charge) is denoted as $\mathrm{GT}_{\mathbb{Z}_{2}}^{4}$. Its excitations are described by a braided fusion 2-category $\Omega^{2} \mathrm{GT}_{\mathbb{Z}_{2}^{f}}^{4}$, which is similar to $\Omega^{2} \mathrm{GT}_{\mathbb{Z}_{2}}^{4}$, except now the $Z_{2}$ charge $e_{p}$ is a fermion. Many results discussed above remain unchanged. In particular, when the $\mathbb{Z}_{2}$ flux strings $m_{s}$ have low energies and $\mathbb{Z}_{2}$ point charges $e_{p}$ have high energies, the system has a low-energy effective categorical symmetry $\mathrm{GT}_{\mathbb{Z}_{2}^{(1)}}^{5}$, as discussed above.

But when $\mathbb{Z}_{2}$ point charges $e_{p}$ have low energies and the $\mathbb{Z}_{2}$ flux strings $m_{s}$ have high energies, the system has a very different behavior since the $\mathbb{Z}_{2}$ point charges are fermions. In this limit, the low-energy excitations are described by fusion 3-category $3 \mathcal{R}$ ep $\mathbb{Z}_{2}^{f}$ (with trivial object, trivial 1-morphism, and $\mathbb{Z}_{2}^{f} 2$-morphisms which contain fermions). The categorical symmetry is $\mathrm{GT}_{\mathbb{Z}_{2}^{f}}^{5}=\operatorname{bulk}\left(3 \mathcal{R}\right.$ ep $\left.\mathbb{Z}_{2}^{f}\right)$ (the $4 \mathrm{~d} \mathbb{Z}_{2}$ gauge theory with fermionic $\mathbb{Z}_{2}$ point charge). What are the gapped liquid phases in a system with $\mathrm{GT}_{\mathbb{Z}_{2}^{f}}^{5}$ categorical symmetry? There is no $3 \mathrm{~d}$ fermionic invertible topological order, so there is only one gapped state (up to stacking of bosonic topological orders with no symmetry) that break the categorical symmetry $\mathrm{GT}_{\mathbb{Z}_{2}^{f}}^{5}$ down to $\mathbb{Z}_{2}$ fermionic 0 -symmetry. There should also be gapless states with the full $\mathrm{GT}_{\mathbb{Z}_{2}^{f}}^{5}$ categorical symmetry.

We like to point out that the $\mathbb{Z}_{2}$ fermionic 0 -symmetry is not an algebraic higher symmetry described by a local fusion higher category $\mathcal{R}$ (i.e., not a bosonic algebraic higher symmetry). The categorical symmetry $\mathrm{GT}_{\mathbb{Z}_{2}^{f}}^{5}$ is not associated with any bosonic algebraic higher symmetries, since $\mathrm{GT}_{\mathbb{Z}_{2}^{f}}^{5} \simeq \operatorname{bulk}(\mathcal{R})$ and $\mathcal{R} \stackrel{\beta}{\rightarrow} 3 \mathcal{V}$ ec has no solution. The $\mathbb{Z}_{2}$ fermionic 0 -symmetry described by $\mathcal{R}=3 \mathcal{R} \operatorname{ep} \mathbb{Z}_{2}^{f}$ satisfies $\mathrm{GT}_{\mathbb{Z}_{2}^{f}}^{5} \simeq \operatorname{bulk}(\mathcal{R})$, but does not satisfy $\mathcal{R} \stackrel{\beta}{\rightarrow} 3 \mathcal{V}$ ec.
One particular realization of the gapped phase is via a Majorana fermion field theory. Here we use a single Weyl fermion field $\psi$ (with two complex components) to describe a single Majorana fermion field (with four real components):

$$
H=\int d^{3} \boldsymbol{x} \psi^{\dagger} i \sigma^{i} \partial_{i} \psi+\left(m \psi^{\top} \epsilon \psi+\text { H.c. }\right),
$$

where $\psi^{\dagger}=\left(\psi^{\top}\right)^{*}$ and $\epsilon \equiv \mathrm{i} \sigma^{2}$. The mass $m$ can be complex. The gapless state at $m=0$ should have the full $\mathrm{GT}_{\mathbb{Z}_{2}^{f}}^{5}$ categorical symmetry. However, it is not clear if it is the minimal gapless state with the full $\mathrm{GT}_{\mathbb{Z}_{2}^{f}}^{5}$ categorical symmetry.

\section{E. $n$ d bosonic systems with $S_{3}$ symmetry}

We consider the class of bosonic $n$ d lattice Hamiltonians $\left\{H_{S_{3}}\right\}$ with $S_{3}=\mathbb{Z}_{3} \rtimes Z_{2}$ symmetry. We also consider the class of boundary Hamiltonians $\left\{H_{S_{3}}^{\text {bndry }}\right\}$ of $(n+1) d S_{3}$ topological order $\mathrm{GT}_{S_{3}}^{n+2}$ with energy gap approaching $\infty$. The class of lattice boundary Hamiltonians $\left\{H_{S_{3}}^{\text {bndry }}\right\}$, by definition, is said to have $\mathrm{GT}_{S_{3}}^{n+2}$ categorical symmetry. We have argued that the class of lattice Hamiltonians $\left\{H_{S_{3}}\right\}$, when restricted to the symmetric subspace, is holo-equivalent to the class of boundary Hamiltonians $\left\{H_{S_{3}}^{\text {bndry }}\right\}$. For example, for each $S_{3}$-symmetric Hamiltonian in the class $\left\{H_{S_{3}}\right\}$, we can find a boundary Hamiltonian in the class $\left\{H_{S_{3}}^{\text {bndry }}\right\}$, such that the two Hamiltonians have the same low-energy properties. In this sense, we say the $S_{3}$-symmetric lattice Hamiltonians also have the $\mathrm{GT}_{S_{3}}^{n+2}$ categorical symmetry. In this section, we ask whether there are other algebraic higher symmetry $\mathcal{R}$, such that the $\mathcal{R}$-symmetric lattice Hamiltonians also have the $\mathrm{GT}_{S_{3}}^{n+2}$ categorical symmetry. In this case, we may say the algebraic higher symmetry $\mathcal{R}$ is holo-equivalent to the $S_{3}$-symmetry (i.e., the $n \mathcal{R} \operatorname{ep} S_{3}$-symmetry).

Certainly, the dual symmetry of the $n \mathcal{R} \operatorname{ep} S_{3}$-symmetry, $n \mathcal{V} \mathrm{ec}_{S_{3}}$, is holo-equivalent to the $n \mathcal{R} \operatorname{ep} S_{3}$-symmetry. Do we have other algebraic higher symmetry $\mathcal{R}$ that is holoequivalent to the $n \mathcal{R} \operatorname{ep} S_{3}$-symmetry?

We first try to solve $\operatorname{bulk}(\mathcal{R})=\operatorname{bulk}\left(n \mathcal{R} \operatorname{ep} S_{3}\right)=\mathrm{GT}_{S_{3}}^{n+2}$ in a physical way. We consider an $(n+1) \mathrm{d} S_{3}$ gauge theory $\mathrm{GT}_{S_{3}}^{n+2}$, whose excitations are described by $\Omega^{2} \mathrm{GT}_{S_{3}}^{n+2}$. The excitations $\mathcal{R}$ on a gapped boundary satisfy $\operatorname{bulk}(\mathcal{R})=\mathrm{GT}_{S_{3}}^{n+2}$.

Let us start with a gauge-flux-condensed boundary, whose excitations are described by $n \mathcal{R} \operatorname{ep} S_{3}$. Such a boundary corresponds to a $S_{3}$-symmetric phase. Next, we try to obtain other boundaries by condensing the point $S_{3}$ charges on this boundary.

The $S_{3}$ charges are described by two bosonic fields: a real field $\sigma$ and a complex field $\phi$. Under the $Z_{2}$ transformation in $S_{3}, \sigma \rightarrow-\sigma$ and $\phi \rightarrow \phi^{*}$. Under the $Z_{3}$ transformation in $S_{3}$, $\sigma \rightarrow \sigma$ and $\phi \rightarrow \mathrm{e}^{\mathrm{i} \frac{2 \pi}{3}} \phi$.

In the first case, we condense the $\sigma$ bosons but not the $\phi$ bosons on the $n \mathcal{R} \operatorname{ep} S_{3}$ boundary by setting $\sigma=$ $1, \phi=0$. This condensation will change the $n \mathcal{R} \operatorname{ep} S_{3}$ boundary to another gapped boundary, denoted as $\mathcal{R}_{\sigma}$. This new boundary $\mathcal{R}_{\sigma}$ corresponds to a $S_{3} \rightarrow Z_{3}$ spontaneous symmetry-breaking phase of $S_{3}$ symmetric systems.

In the second case, we condense the $\phi$ bosons but not the $\sigma$ bosons on the $n \mathcal{R e p} S_{3}$ boundary, by setting $\phi=1, \sigma=0$. 
This condensation will change the $n \mathcal{R}$ ep $S_{3}$ boundary to a gapped boundary, denoted as $\mathcal{R}_{\phi}$. The boundary $\mathcal{R}_{\phi}$ corresponds to a $S_{3} \rightarrow Z_{2}$ spontaneous symmetry breaking-phase of $S_{3}$ symmetric systems.

Does $\mathcal{R}_{\sigma}$ describe the algebraic higher symmetry in the $S_{3} \rightarrow Z_{3}$ spontaneous symmetry breaking phase? That is, can the $S_{3} \rightarrow Z_{3}$ spontaneous symmetry-breaking phase be viewed as the trivial symmetric phase of the $\mathcal{R}_{\sigma}$-symmetry? Also, does $\mathcal{R}_{\phi}$ describe the algebraic higher symmetry in the $S_{3} \rightarrow Z_{2}$ spontaneous symmetry-breaking phase?

Although $\mathcal{R}_{\sigma}$ and $\mathcal{R}_{\phi}$ satisfy $\operatorname{bulk}\left(\mathcal{R}_{\sigma}\right)=\operatorname{bulk}\left(\mathcal{R}_{\phi}\right)=$ $\mathrm{GT}_{S_{3}}^{n+2}$, we still need to show they are local fusion higher categories in order for them to describe algebraic higher symmetries. For this purpose, we start with the $n \mathrm{~d} S_{3}$-gauge theory constructed by stacking two $n \mathcal{R} \operatorname{ep} S_{3}$ via their common bulk $\mathrm{GT}_{S_{3}}^{n+2}\left[\mathrm{a}(n+1) \mathrm{d} S_{3}\right.$-gauge theory], as shown in Fig. 5. The excitations in the $n \mathrm{~d} S_{3}$-gauge theory are given by $n \mathcal{R} \operatorname{ep} S_{3} \underset{\mathrm{GT}_{S_{3}}^{n+2}}{\otimes}\left(n \mathcal{R} \operatorname{ep} S_{3}\right)^{\text {rev }}$. Now we condense $\sigma$ on one boundary and condense $\phi$ on the other boundary. The resulting $n \mathrm{~d}$ state has excitations given by $\mathcal{R}_{\sigma} \underset{\mathrm{GT}_{s_{3}}^{n+2}}{\otimes} \mathcal{R}_{\phi}^{\text {rev }}$. From our physical understanding, when both $\sigma$ and $\phi$ condense, the $S_{3}$-gauge symmetry is completely broken, and the $n$ d topological order described by the $S_{3}$-gauge theory becomes a trivial phase. This implies that $\mathcal{R}_{\sigma} \underset{\mathrm{GT}_{S_{3}+2}^{+2}}{\otimes} \mathcal{R}_{\phi}^{\text {rev }}=n \mathcal{V}$ ec. Then using Proposition 39, we find that $\mathcal{R}_{\sigma}$ and $\mathcal{R}_{\phi}$ are both local fusion higher categories. They describe a pair of dual algebraic higher symmetries.

\section{ACKNOWLEDGMENTS}

L.K. and H.Z. are supported by the Science, Technology, and Innovation Commission of Shenzhen Municipality (Grant No. ZDSYS20170303165926217) and by Guangdong Provincial Key Laboratory (Grant No. 2019B121203002). L.K. is also supported by NSFC under Grant No. 11971219. X.G.W. is partially supported by NSF DMS- 1664412 and by the Simons Collaboration on Ultra-quantum Matter, which is a grant from the Simons Foundation (651440). H.Z. is also supported by NSFC under Grant No. 11871078.
[1] G. 't Hooft, in Recent Developments in Gauge Theories, edited by G. Hooft et al., NATO Advanced Study Institutes Series (Series B. Physics) Vol. 59 (Springer, Boston, MA, 1980), pp. 135-157.

[2] X. Chen, Z.-C. Gu, Z.-X. Liu, and X.-G. Wen, Phys. Rev. B 87, 155114 (2013).

[3] X.-G. Wen, Phys. Rev. D 88, 045013 (2013).

[4] D. Gaiotto, A. Kapustin, N. Seiberg, and B. Willett, J. High Energy Phys. 02 (2015) 172.

[5] A. Kitaev, Ann. Phys. 303, 2 (2003).

[6] X.-G. Wen, Phys. Rev. Lett. 90, 016803 (2003).

[7] M. Levin and X.-G. Wen, Phys. Rev. B 67, 245316 (2003).

[8] B. Yoshida, Ann. Phys. 326, 15 (2011).

[9] H. Bombín, Commun. Math. Phys. 327, 387 (2014).

[10] Z. Nussinov and G. Ortiz, Proc. Natl. Acad. Sci. USA 106, 16944 (2009).

[11] Z. Nussinov and G. Ortiz, Ann. Phys. 324, 977 (2009).

[12] M. B. Hastings and X.-G. Wen, Phys. Rev. B 72, 045141 (2005).

[13] A. Kapustin and R. Thorngren, in Algebra, Geometry, and Physics in the 21st Century, edited by D. Auroux, L. Katzarkov, T. Pantev, Y. Soibelman, and Y. Tschinkel, Progress in Mathematics Vol. 324 (Birkhäuser, Cham, 2017), pp. 177-202.

[14] R. Thorngren and C. von Keyserlingk, arXiv:1511.02929.

[15] A. Bullivant, M. Calcada, Z. Kádár, P. Martin, and J. F. Martins, Rev. Math. Phys. 32, 2050011 (2020).

[16] X.-G. Wen, Phys. Rev. B 99, 205139 (2019).

[17] Z. Wan and J. Wang, Ann. Math. Sci. Appl. 4, 107 (2019).

[18] L. Tsui and X.-G. Wen, Phys. Rev. B 101, 035101 (2020).

[19] W. Ji and X.-G. Wen, Phys. Rev. Res. 2, 033417 (2020).

[20] J. Fröhlich, J. Fuchs, I. Runkel, and C. Schweigert, Nucl. Phys. B 763, 354 (2007).

[21] A. Davydov, L. Kong, and I. Runkel, Adv. Theor. Math. Phys. 15, 43 (2011).

[22] C.-M. Chang, Y.-H. Lin, S.-H. Shao, Y. Wang, and X. Yin, J. High Energy Phys. 01 (2019) 026.

[23] R. Thorngren and Y. Wang, arXiv:1912.02817.
[24] W. Ji and X.-G. Wen, Phys. Rev. Res. 1, 033054 (2019).

[25] L. Kong and X.-G. Wen, arXiv:1405.5858.

[26] L. Kong, X.-G. Wen, and H. Zheng, arXiv:1502.01690.

[27] L. Kong, X.-G. Wen, and H. Zheng, Nucl. Phys. B 922, 62 (2017).

[28] T. Lan and X.-G. Wen, Phys. Rev. X 9, 021005 (2019).

[29] D. Gaiotto and T. Johnson-Freyd, J. High Energy Phys. 05 (2019) 007.

[30] T. Johnson-Freyd, (2020), arXiv:2003.06663.

[31] L. Kong, T. Lan, X.-G. Wen, Z.-H. Zhang, and H. Zheng, arXiv:2005.14178.

[32] X. Chen, Z.-C. Gu, and X.-G. Wen, Phys. Rev. B 82, 155138 (2010).

[33] A. Kitaev, Ann. Phys. 321, 2 (2006).

[34] M. A. Levin and X.-G. Wen, Phys. Rev. B 71, 045110 (2005).

[35] T. Lan and X.-G. Wen, Phys. Rev. B 90, 115119 (2014).

[36] X.-G. Wen, Phys. Rev. B 40, 7387 (1989).

[37] X.-G. Wen and Q. Niu, Phys. Rev. B 41, 9377 (1990).

[38] X.-G. Wen, Int. J. Mod. Phys. B 04, 239 (1990).

[39] B. Zeng and X.-G. Wen, Phys. Rev. B 91, 125121 (2015).

[40] B. Swingle and J. McGreevy, Phys. Rev. B 93, 045127 (2016).

[41] D. Freed, arXiv:1406.7278.

[42] A. Kapustin, arXiv:1404.6659.

[43] T. Tannaka, Tohoku Math. J. 45, 1 (1938).

[44] N. Ganter and M. Kapranov, Adv. Math. 217, 2268 (2008).

[45] T. Lan, L. Kong, and X.-G. Wen, Commun. Math. Phys. 351, 709 (2016).

[46] T. Lan, L. Kong, and X.-G. Wen, Phys. Rev. B 95, 235140 (2017).

[47] Z.-C. Gu, Z. Wang, and X.-G. Wen, Phys. Rev. B 91, 125149 (2015).

[48] T. Lan, L. Kong, and X.-G. Wen, Phys. Rev. B 94, 155113 (2016).

[49] L. Bhardwaj, D. Gaiotto, and A. Kapustin, J. High Energy Phys. 04 (2017) 096.

[50] Z.-C. Gu and X.-G. Wen, Phys. Rev. B 90, 115141 (2014). 
[51] A. Kapustin, R. Thorngren, A. Turzillo, and Z. Wang, J. High Energy Phys. 12 (2015) 1.

[52] C. Wang and T. Senthil, Phys. Rev. B 89, 195124 (2014).

[53] D. Gaiotto and A. Kapustin, Int. J. Mod. Phys. A 31, 1645044 (2016).

[54] D. Freed and M. Hopkins, arXiv:1604.06527.

[55] Q.-R. Wang and Z.-C. Gu, Phys. Rev. X 8, 011055 (2018).

[56] A. Kapustin and R. Thorngren, J. High Energy Phys. 10 (2017) 080.

[57] T. Lan, C. Zhu, and X.-G. Wen, Phys. Rev. B 100, 235141 (2019).

[58] M. Guo, K. Ohmori, P. Putrov, Z. Wan, and J. Wang, Commun. Math. Phys. 376, 1073 (2020).

[59] A. Kitaev and L. Kong, Commun. Math. Phys. 313, 351 (2012).

[60] M. Levin, Commun. Math. Phys. 378, 1081 (2020).

[61] L. Kong and H. Zheng, J. High Energy Phys. 02 (2020) 150.

[62] L. Kong and H. Zheng, arXiv:1912.01760.

[63] J. Maldacena, Adv. Theor. Math. Phys. 2, 231 (1998).

[64] E. Witten, Adv. Theor. Math. Phys. 2, 253 (1998).

[65] D. Harlow and H. Ooguri, arXiv:1810.05338.

[66] R. Dijkgraaf and E. Witten, Commun. Math. Phys. 129, 393 (1990).

[67] C. Zhu, T. Lan, and X.-G. Wen, Phys. Rev. B 100, 045105 (2019).

[68] L. Kong, T. Lan, X.-G. Wen, Z.-H. Zhang, and H. Zheng, J. High Energy Phys. 09 (2020) 093.

[69] C. Goff, G. Mason, and S.-H. Ng, J. Algebra 312, 849 (2007).

[70] J. C. Wang and X.-G. Wen, Phys. Rev. B 91, 035134 (2015).

[71] Z.-C. Gu and X.-G. Wen, Phys. Rev. B 80, 155131 (2009).

[72] S.-P. Kou and X.-G. Wen, Phys. Rev. B 80, 224406 (2009).

[73] Y.-M. Lu and A. Vishwanath, Phys. Rev. B 93, 155121 (2016).

[74] A. Mesaros and Y. Ran, Phys. Rev. B 87, 155115 (2013).

[75] L.-Y. Hung and Y. Wan, Phys. Rev. B 87, 195103 (2013).

[76] C. Xu, Phys. Rev. B 88, 205137 (2013).
[77] L.-Y. Hung and Y. Wan, Int. J. Mod. Phys. B 28, 1450172 (2014).

[78] L. Chang, M. Cheng, S. X. Cui, Y. Hu, W. Jin, R. Movassagh, P. Naaijkens, Z. Wang, and A. Young, J. Phys. A: Math. Theor. 48, 12FT01 (2015).

[79] M. Cheng, Z.-C. Gu, S. Jiang, and Y. Qi, Phys. Rev. B 96, 115107 (2017).

[80] C. Heinrich, F. Burnell, L. Fidkowski, and M. Levin, Phys. Rev. B 94, 235136 (2016).

[81] N. Read and S. Sachdev, Phys. Rev. Lett. 66, 1773 (1991).

[82] X.-G. Wen, Phys. Rev. B 44, 2664 (1991).

[83] A. Y. Kitaev, Phys. Usp. 44, 131 (2001).

[84] M. Levin, Phys. Rev. X 3, 021009 (2013).

[85] X.-G. Wen, Phys. Rev. B 95, 205142 (2017).

[86] X. Chen, Z.-X. Liu, and X.-G. Wen, Phys. Rev. B 84, 235141 (2011).

[87] A. Vishwanath and T. Senthil, Phys. Rev. X 3, 011016 (2013).

[88] X. Chen, Z.-C. Gu, and X.-G. Wen, Phys. Rev. B 83, 035107 (2011).

[89] N. Schuch, D. Pérez-García, and I. Cirac, Phys. Rev. B 84, 165139 (2011).

[90] M. Barkeshli, P. Bonderson, M. Cheng, and Z. Wang, Phys. Rev. B 100, 115147 (2019).

[91] T. Lan, L. Kong, and X.-G. Wen, Phys. Rev. X 8, 021074 (2018).

[92] A. Davydov and D. A. Simmons, Homol., Homotopy Appl. 20, 275 (2018).

[93] T. Lan, J. C. Wang, and X.-G. Wen, Phys. Rev. Lett. 114, 076402 (2015).

[94] T. Lan, X. Wen, L. Kong, and X.-G. Wen, Phys. Rev. Res. 2, 023331 (2020).

[95] N. Read and D. Green, Phys. Rev. B 61, 10267 (2000).

[96] X.-G. Wen, Phys. Rev. Lett. 84, 3950 (2000). 